Florida International University FIU Digital Commons

2-2-2010

\title{
The Construction and Optimization on an Ion Mobility Spectrometer for the Analysis of Explosives and Drugs
}

Hanh Tuyet Lai

Florida International University, hlai001@fiu.edu

DOI: $10.25148 /$ etd.FI10041618

Follow this and additional works at: https:// digitalcommons.fiu.edu/etd

Part of the Analytical Chemistry Commons

\section{Recommended Citation}

Lai, Hanh Tuyet, "The Construction and Optimization on an Ion Mobility Spectrometer for the Analysis of Explosives and Drugs" (2010). FIU Electronic Theses and Dissertations. 169.

https://digitalcommons.fiu.edu/etd/169 


\title{
FLORIDA INTERNATIONAL UNIVERSITY
}

Miami, Florida

THE CONSTRUCTION AND OPTIMIZATION OF AN ION MOBILITY SPECTROMETER FOR THE ANALYSIS OF EXPLOSIVES AND DRUGS

A dissertation submitted in partial fulfillment of the requirements for the degree of DOCTOR OF PHILOSOPHY

\author{
in \\ CHEMISTRY
}

by

Hanh Tuyet Lai

2010 
To: Dean Kenneth Furton

College of Arts and Sciences

This dissertation, written by Hanh Tuyet Lai, and entitled The Construction and Optimization of an Ion Mobility Spectrometer for the Analysis of Explosives and Drugs, having been approved in respect to style and intellectual content, is referred to you for judgment.

We have read this dissertation and recommend that it be approved.

Bruce McCord

Surendra K. Saxena

Konstantinos Kavallieratos

Kenneth Furton

José R. Almirall, Major Professor

Date of Defense: February 2, 2010

The dissertation of Hanh Tuyet Lai is approved.

Dean Kenneth Furton

College of Arts and Sciences

Interim Dean Kevin O'Shea

University Graduate School

Florida International University, 2010 
(C) Copyright 2010 by Hanh Tuyet Lai All rights reserved. 


\section{DEDICATION}

I dedicate this dissertation to my family, especially to my mom and dad whom many years ago left their mother country with two pairs of empty hands, and brought their children to the land of opportunities. It is because of them that today I have the chance to study and follow my dreams. I could not be where I am today without their love, hard work, support, and sacrifice. They are my existence and my success will forever be theirs.

I would like to dedicate this work to all the friends in my personal and professional network, who have always welcomed me with their kind word, kind thought, and kind heart. Last but not least, I dedicate this dissertation to my special friend Michael, who has been by my side throughout the hardest years, to whom I can share my true emotions especially in the down times. His unconditional love, support, trust, and confidence in me has helped and will continue to help me overcome many tough waves in life. 


\section{ACKNOWLEDGMENTS}

The successful completion of my dissertation I wish to thank the members of my committee for their support, and guidance throughout the last 4 years. Special thanks to my major professor, Dr. José R. Almirall for his endless support, his confidence in my abilities, and for all the opportunities and resources he has given me to learn, grow, and shine.

I would like to express my appreciation for Dr. Jill R. Scott, whose presence brought me the opportunity to take my dissertation project in the direction I always wanted, and gain some of the most valuable skills as a scientist while honing my skills as an engineer. Many thanks to Dr. Nathan Leigh, Mr. Norman Huang, and Mr. Richard Zicarelli for their patience in teaching me the necessary knowledge in software, electronics, and machining that I needed in order to successfully complete the fifth task in my dissertation project. I am very thankful for their voluntarily advice and services.

I would like to acknowledge the FIU University Graduate School for awarding me the Dissertation Year Fellowship, as well as the FIU Entrepreneur Center for support I received through the Kauffman Entrepreneurial Doctoral Student Fellowship. I am also thankful for Smiths Detection and General Electric for the donations of the instruments, and the DHS Transportation Security Laboratory, the Idaho National Laboratory, and the Miami Dade Police Department for providing access to their facilities as well as the support from their teams for me to conduct my experiments. I also would like to thanks the Journal of Separation Sciences, Analytical and Bioanalytical Chemistry, and International Journal of Mass Spectrometry for permitting the re-use of my publications' contents for this dissertation.

Finally, I would like to acknowledge the former and present group members of the Almirall group for their friendship and professionalism, some of whom will remain my lifelong friends and colleagues in the endeavor of bringing new discoveries into the world of science. 


\title{
ABSTRACT OF THE DISSERTATION \\ THE CONSTRUCTION AND OPTIMIZATION OF AN ION MOBILITY SPECTROMETER FOR THE ANALYSIS OF EXPLOSIVES AND DRUGS
}

by

\author{
Hanh Tuyet Lai
}

Florida International University, 2010

Miami, Florida

Professor José R. Almirall, Major Professor

Today, over 15,000 Ion Mobility Spectrometry (IMS) analyzers are employed at worldwide security checkpoints to detect explosives and illicit drugs. Current portal IMS instruments and other electronic nose technologies detect explosives and drugs by analyzing samples containing the headspace air and loose particles residing on a surface. Canines can outperform these systems at sampling and detecting the low vapor pressure explosives and drugs, such as RDX, PETN, cocaine, and MDMA, because these biological detectors target the volatile signature compounds available in the headspace rather than the non-volatile parent compounds of explosives and drugs.

In this dissertation research volatile signature compounds available in the headspace over explosive and drug samples were detected using SPME as a headspace sampling tool coupled to an IMS analyzer. A Genetic Algorithm (GA) technique was developed to optimize the operating conditions of a commercial IMS (GE Itemizer 2), leading to the successful detection of plastic explosives (Detasheet, Semtex H, and C-4) and illicit drugs (cocaine, MDMA, and marijuana). Short sampling times (between $10 \mathrm{sec}$ to $5 \mathrm{~min}$ ) were adequate to extract and preconcentrate sufficient analytes $(>20 \mathrm{ng}$ ) representing the volatile signatures in the headspace of a $15 \mathrm{~mL}$ glass vial or a quart-sized can containing $\leq 1 \mathrm{~g}$ of the bulk explosive or drug. 
Furthermore, a research grade IMS with flexibility for changing operating conditions and physical configurations was designed and fabricated to accommodate future research into different analytes or physical configurations. The design and construction of the FIU-IMS were facilitated by computer modeling and simulation of ion's behavior within an IMS. The simulation method developed uses SIMION/SDS and was evaluated with experimental data collected using a commercial IMS (PCP Phemto Chem 110). The FIU-IMS instrument has comparable performance to the GE Itemizer 2 (average resolving power of 14, resolution of 3 between two drugs and two explosives, and LODs range from 0.7 to $9 \mathrm{ng}$ ).

The results from this dissertation further advance the concept of targeting volatile components to presumptively detect the presence of concealed bulk explosives and drugs by SPME-IMS, and the new FIU-IMS provides a flexible platform for future IMS research projects. 


\section{TABLE OF CONTENTS}

CHAPTER

PAGE

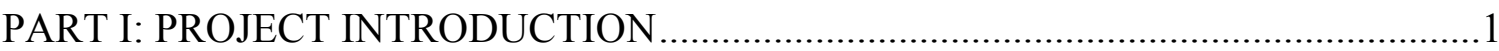

CHAPTER 1: PROJECT MOTIVATION \& SIGNIFICANCE ................................................. 1

CHAPTER 2: CURRENT CHALLENGES \& FUTURE SOLUTIONS .......................................... 2

2.1 Current Challenge in Detection of Explosives and Illicit Drugs................................... 2

2.2 Future Solutions: Research Hypotheses.................................................................... 3

CHAPTER 3: RESEARCH APPROACH \& PROJECT DELIVERABLES ................................. 4

3.1 Targeting the Volatile Signature Compounds of Drugs and Explosives ....................... 4

3.2 Systematic Optimization for Method Development ................................................... 4

3.3 Modeling and Simulation for Instrumental Design..................................................... 5

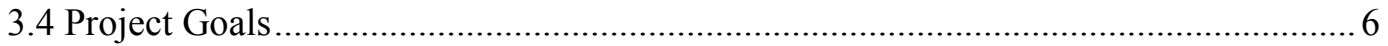

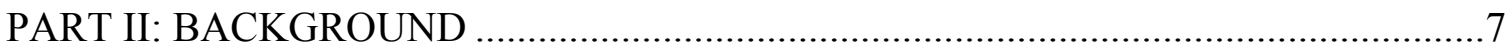

CHAPTER 4: THE CHEMISTRY AND DETECTION OF HIDDEN EXPLOSIVES ................. 7

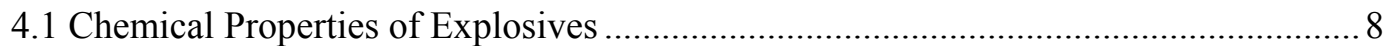

4.2 Detection Technologies for Explosives ................................................................. 12

4.3 Effects of Chemical and Physical Properties of Explosives on Detection................... 18

4.4 Trace detection challenges.................................................................................... 19

4.5 Detection of Taggants and Volatile Chemical Components of Explosives ................. 20

CHAPTER 5: THE CHEMISTRY AND DETECTION OF ILLICIT DRUGS ........................... 23

5.1 Chemistry of Illicit Drugs and Compounds of Interest ...............................................24

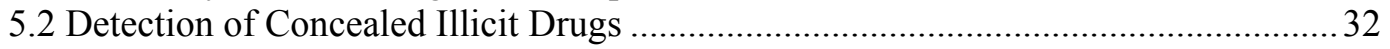

CHAPTER 6: SAMPLING AND PRECONCENTRATION DEVICES …............................... 37

6.1 Desired Characteristics of Preconcentration Devices ……............................................ 38

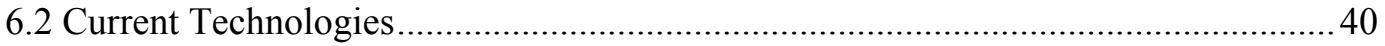

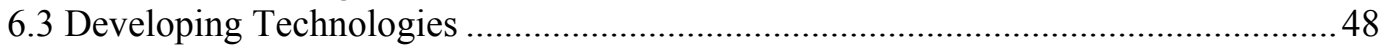

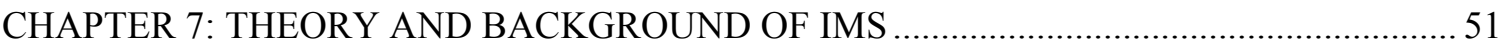

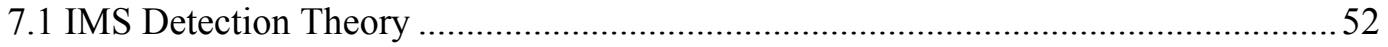

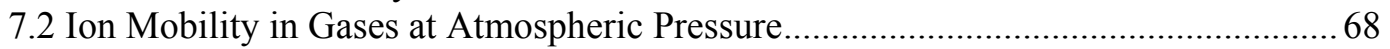

7.3 Operational Parameters Affecting IMS performance ……........................................ 76

7.4 IMS Limit of detection, Linear Dynamic Range, and \% RSD..................................... 82

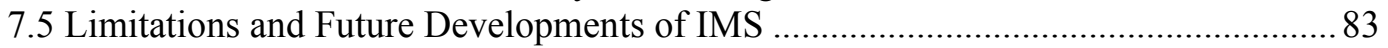

CHAPTER 8: COMPUTER SIMULATION FOR THE DESIGN OF ION MOBILITY

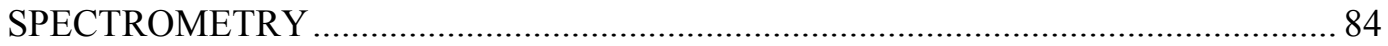

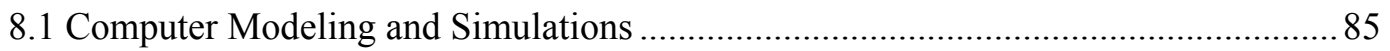

8.2 Challenges of Modeling Ion in Gas at Atmospheric Pressure .................................... 86

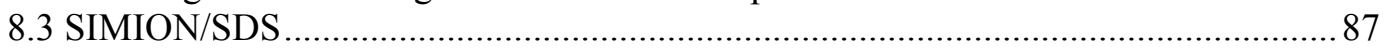

PART III: MATERIALS AND METHODS …...................................................... 88 
CHAPTER 9: METHOD DEVELOPMENT FOR DETECTION OF DRUGS AND

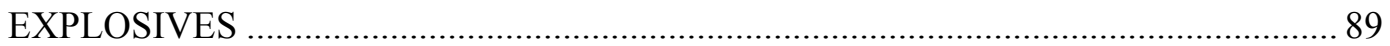

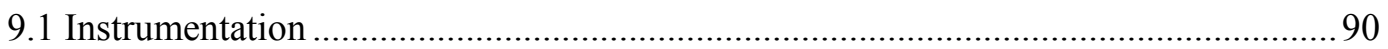

9.2 IMS Optimization for New Compounds of Interest............................................... 90

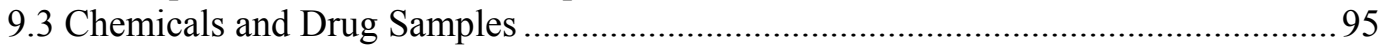

9.4 SPME Extraction for Volatile Components of Drugs .................................................98

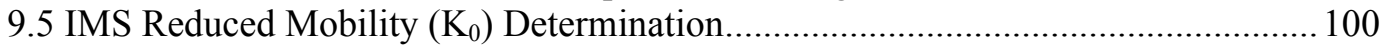

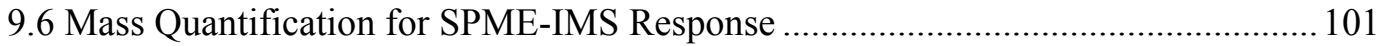

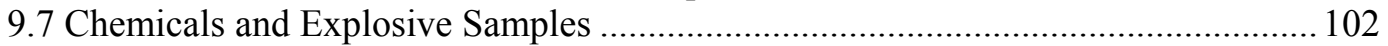

CHAPTER 10: METHOD DEVELOPMENT FOR IMS MODELING AND SIMULATIONS 106

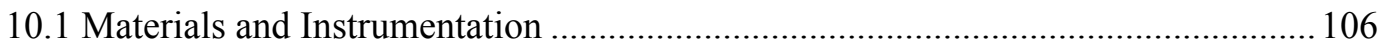

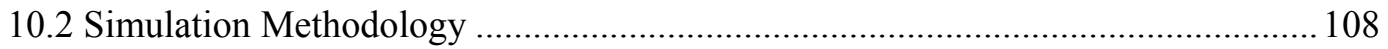

10.3 Evaluation of Simulation Method: Empirical vs. Simulation Experiments ............. 115

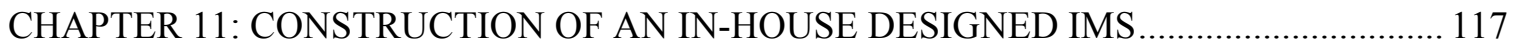

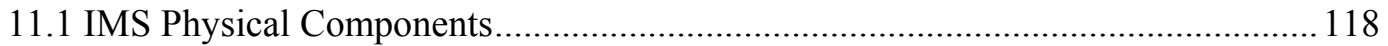

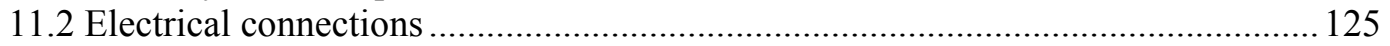

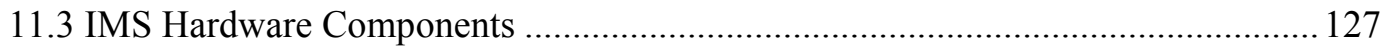

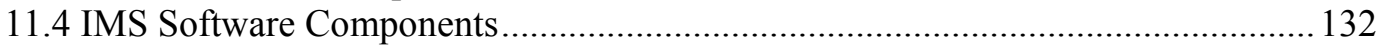

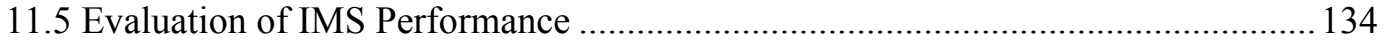

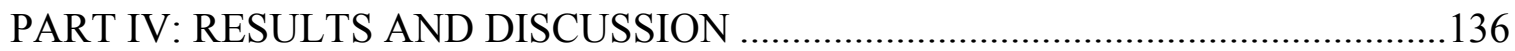

CHAPTER 12: DETECTION OF VOLATILE COMPONENTS OF ILLICIT DRUGS BY

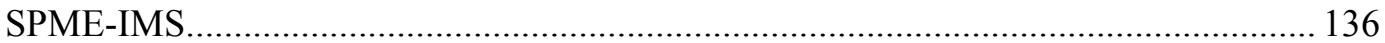

12.1 Plasmagrams of the newly detected compounds................................................ 136

12.2 Detection Limits, Repeatability and Linear Dynamic Range of SPME-IMS .......... 141

12.3 SPME Extraction Profile at Equilibrium for model compound DMNB ................. 144

12.4 Optimization of IMS conditions using a Genetic Algorithm................................146

12.5 Headspace sampling of cocaine, MDMA, and marijuana...................................... 149

CHAPTER 13: DETECTION OF VOLATILE COMPONENTS OF EXPLOSIVES BY

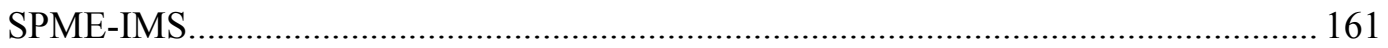

13.1 Headspace SPME-GC/MS analysis of Detasheet, Semtex H, and C-4 ................... 162

13.2 Headspace SPME-IMS analysis of Detasheet, Semtex H, and C-4 ...................... 168

CHAPTER 14: EVALUATION OF SIMION/SDS SIMULATION METHOD OF

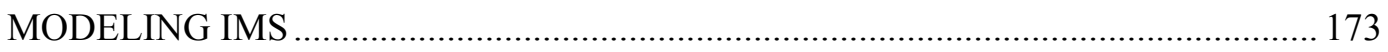

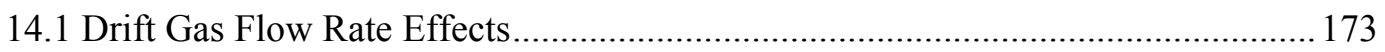

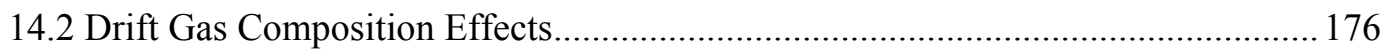

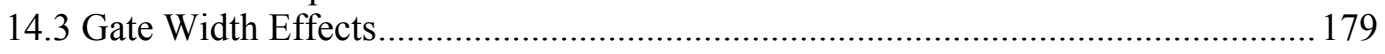

CHAPTER 15: DETECTION FIGURES OF MERIT FOR IN-HOUSE FABRICATED IMS .. 183

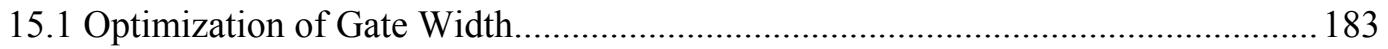

15.2 Optimization of Electric Field at Different Region of IMS .................................. 184

15.3 FIU IMS Responses of Common Explosives and Illicit Drugs .............................. 190

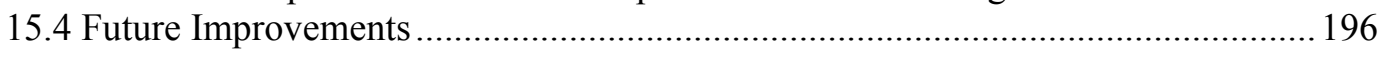

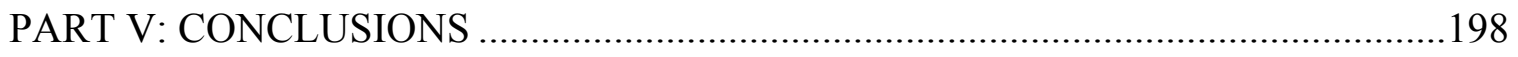




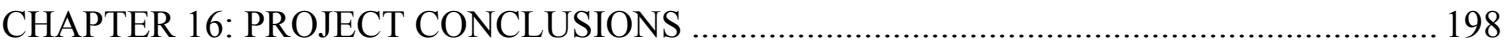

16.1 Improving Detection of Hidden Explosives........................................................... 198

16.2 Improving Detection of Hidden Illicit Drugs........................................................199

16.3 Computer Modeling and Simulation Method for IMS Instrumental Design ........... 199

16.4 Design and Construction of an IMS with Computer Controls ................................200

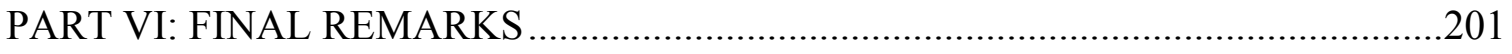

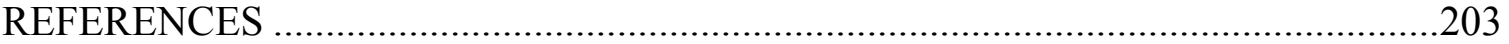

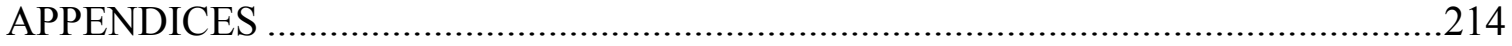

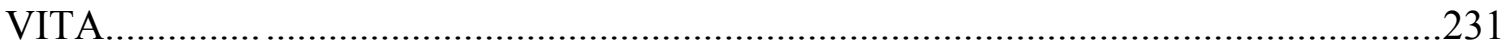




\section{LIST OF TABLES}

TABLE

Table 1. Composition of low explosives and structure of high explosives.................................. 9

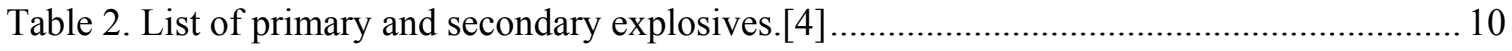

Table 3. Chemical composition of plastic explosives, C-4, Detasheet, and Semtex A/H............. 11

Table 4. The molecular structure, vapor pressure, and minimum concentration of taggants used in the marking low vapor pressure plastic explosives........................................ 21

Table 5. Federal scheduling of controlled substances in the US.[38] ........................................ 26

Table 6. Impurity profiles of MDMA reported in various studies found in literature. .................. 28

Table 7. Chemical structure and vapor pressure of impurities found in headspace air above MDMA

Table 8. Chemical structure and vapor pressure of impurities reported in headspace air of cocaine.

Table 9. Chemical structure, and $\mathrm{P}_{\mathrm{v}}$ of dominant volatile constituents from marijuana.

Table 10. Commercially available SPME fiber coatings. 48

Table 11. Performance of various ionization sources for IMS detection systems.

Table 12. Reduced mobility values of some common explosives (detected in negative mode) and drugs (detected in positive mode).[17] ................................................................ 76

Table 13. Experimental setup and encoding of IMS settings for binary representation.

Table 14. Dominant headspace components and corresponding vapor pressures of the parent compounds of interest.

Table 15. Details of the plastic explosive samples. 103

Table 16. Operating conditions of IMS, SPME interface, and GC/MS. 104

Table 17. IMS conditions for gas flow rate, drift gas composition, and gate width study. 108

Table 18. Reduced mobilities of volatile components at the corresponding operating condition.

Table 19. LODs and LDRs calculated for SPME-IMS and direct IMS from their corresponding response curve. 
Table 20. Location of the interference peaks with respect to methyl benzoate for samples containing cocaine and interference product.

Table 21. Location of the interference peaks with respect to piperonal for samples containing MDMA and interference product.

Table 22. Location of the interference peaks with respect to (limonene, $\alpha / \beta$-pinene) for samples containing marijuana and interference product..

Table 23. Summary of the volatile components detected by SPME-GC/MS(x) \& SPME-IMS at one universal setting (a).

Table 24. Reduced mobility values $\left(\mathrm{K}_{0}\right)$ and Limits of Detection by IMS.

Table 25. FIU-IMS performance summary of the analysis of explosives $\left(180{ }^{\circ} \mathrm{C} \mathrm{CH}_{2} \mathrm{Cl}_{2}\right.$ dopant), and illicit drugs $\left(180^{\circ} \mathrm{C} \mathrm{NH}_{3}\right.$ dopant). 


\section{LIST OF FIGURES}

\section{FIGURE}

Figure 1. Content flowchart on background of explosives. ................................................... 8

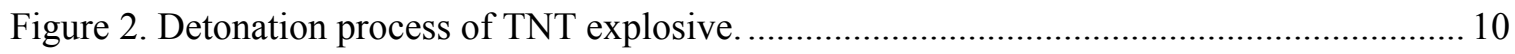

Figure 3. Content flow chart of background on explosive detection technologies....................... 12

Figure 4. Number of canine teams utilized at airports and mass transit systems (2000-2007).[8] 15

Figure 5. Detection limits of trace explosive detection systems compared to IMS detector for TNT model compound.

Figure 6. The DEA 2008 drugs statistics on the most common drugs seized in the US (not

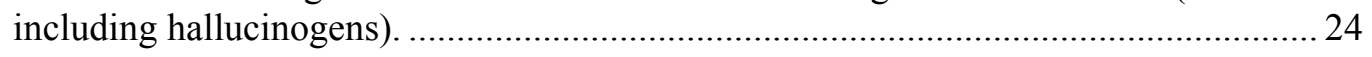

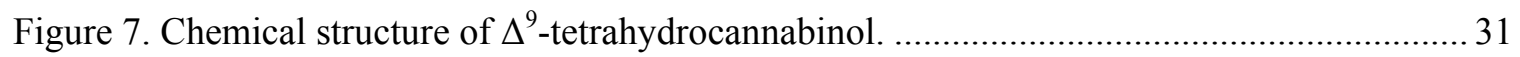

Figure 8. Relative performance of fiber SPME device compared to other devices based on pre-concentration factor and cost of manufacture................................................... 42

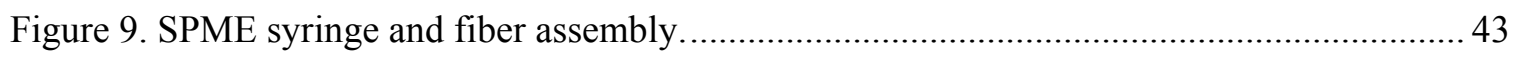

Figure 10. Headspace SPME extraction vs. Direct SPME extraction showing the concentration profiles of the analyte between phases with respect to distance.[64] ............................ 46

Figure 11. Schematic of a low field conventional stacked IMS and the ion mobility coefficient. 53

Figure 12. Electrical fields at the Bradbury-Nielsen gate created by the offset in voltage between the two sets of wire.

Figure 13. Deflection angle of ions in proximity of the Bradbury-Nielsen gate.[87]

Figure 14. Formation of positive and negative product ions occur within the ${ }^{63} \mathrm{Ni}$ ionization source in supporting atmosphere at ambient pressure............................................... 71

Figure 15. Electronegativity of some selected chemical classes............................................... 72

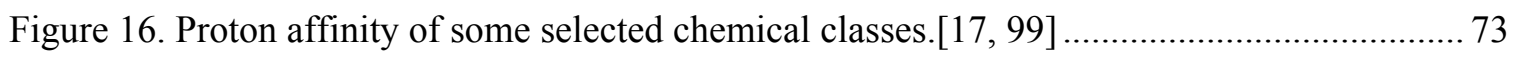

Figure 17. SPME-IMS interface and GE Itemizer 2 ........................................................ 90

Figure 18. Substance window in nartcotic mode (GE Itemiser 2)........................................... 91

Figure 19. Schematic of optimization using a Genetic Algorithm.......................................... 92

Figure 20. 10 cans sealed for MDMA blind study of which some contain MDMA as well as interference products. 98 


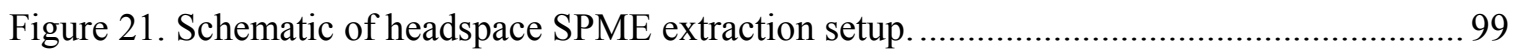

Figure $22 . \mathrm{K}_{0}$ determination from an analysis of the standard using the Smiths IMS................. 100

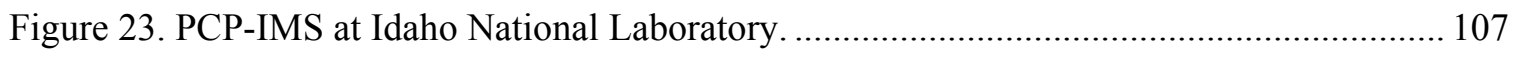

Figure 24. 3D computer model of PCP IMS showing the gas trajectories from the Fluid Dynamic Simulation..

Figure 25. PCP IMS's dimensions (in $\mathrm{mm}$ ) on 2D drawing extracted from SodidWorks drawing.

Figure 26. SIMION isometric view (i), and SIMION potential energy field view (ii) of the PCP IMS

Figure 27. Simulated ion peak from histogram after spline-fitted.

Figure 28. 3D Autodesk Inventor exploded-view of the IMS physical components assembly ... 118

Figure 29. FIU-IMS aluminum inlet.

Figure 30. GE desorber and SPME interface with aluminum inlet......

Figure $31 .{ }^{63} \mathrm{Ni}$ ionization source (Nickel foil dimension) inserted inside a conductive ring...... 120

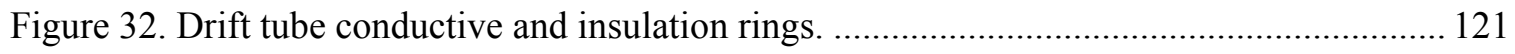

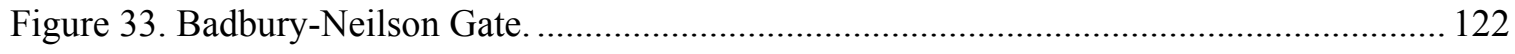

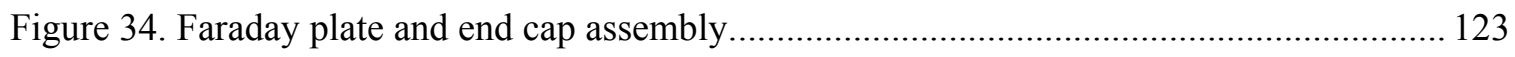

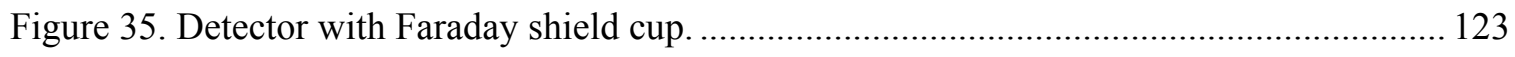

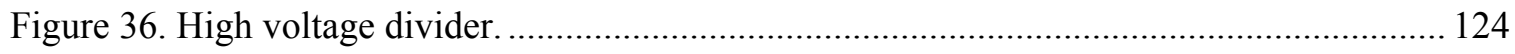

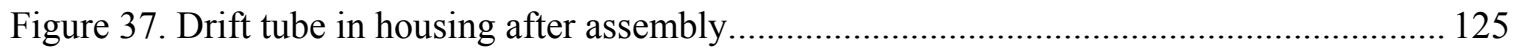

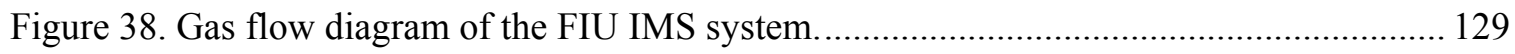

Figure 39. HV drift tube power supply/ion shutter switch box and Keithley amplifier. ............ 130

Figure 40. BNC connector box to DAQ card on IMS computer. ........................................... 131

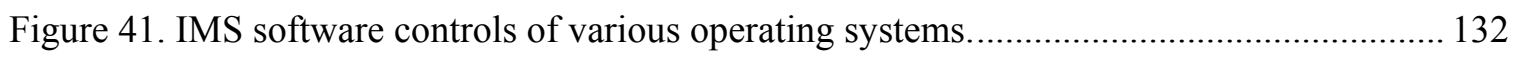

Figure 42. IMS spectra of piperonal, and methyl benzoate overlayed on blank fiber extraction at optimized operating condition........................................................................ 138

Figure 43. IMS spectra of limonene, $\alpha$-pinene, and $\beta$-pinene overlayed on blank fiber extraction at optimized condition. 
Figure 44. 3D plasmagram, plot of $\beta$-pinene's monomer and proton bound dimer.

Figure 45. SPME-IMS response curve of (i) Piperonal, (ii) Methyl Benzoate, (iii) Limonene,

(iv) $\alpha$-pinene, (v) $\beta$-pinene at individually optimized IMS condition.

Figure 46. Response Curves by direct headspace volume injection into IMS. (i): Piperonal, (ii): Methyl Benzoate, (iii): Limonene, (iv): $\alpha$-pinene, (v): $\beta$-pinene at individually optimized IMS condition.

Figure 47. (i) SPME-IMS extraction equilibrium time curve, (ii) headspace equilibrium time for DMNB model compound.

Figure 48. Comparison of IMS configurations' responses obtained by GA (i) versus random (ii), and GA as precursor to SIMPLEX (ii) for DMNB.

Figure 49. Convergence of GA optimization search after six generations for DMNB test compound.

Figure 50. Overlay spectrum of a five-minute SPME headspace extraction of standards vs. actual drugs; (i) cocaine $\mathrm{HCl}$, (ii) MDMA, and (iii) marijuana.

Figure 51. 3D plasmagram of a 30-minute SPME headspace extraction of cocaine $\mathrm{HCl}$.

Figure 52. Gas chromatogram of a 30-minute SPME extraction of cocaine $\mathrm{HCl}$ sample inside

a glass vial.

Figure 53. Formation of methyl benzoate (path a) and ecgonidine methyl ester (path b) from cocaine decomposition.

Figure 54. SPME-IMS extraction time profiles for (i) cocaine, (ii) MDMA, and

(iii) marijuana. Compounds detected are methyl benzoate, piperonal and

terpenes/caryophyllene, respectively at individually optimized condition.

Figure 55. Piperonal alarm result for blind study of MDMA and interference products.

Figure 56. Chromatogram of (i) untagged Detasheet, and (ii) tagged Primasheet explosives.

(1) acetic acid; (2) 1-butanol; (3) toluene; (4) n-butyl acetate; (5) taggant DMNB..... 163

Figure 57. Comparison of different fiber chemistry for headspace extraction of (i) Flex X, 20 min. (ii) Semtex H, 20 min., and (iii) C-4, 5 min by GC/MS.

Figure 59. SPME-IMS extraction time curves of (i) Flex X, (ii) Semtex H, and (iii) C-4 at optimized condition.

Figure 60. SPME-IMS extraction time curves of (i) Flex X, (ii) Semtex H, and (iii) C-4 at optimized condition.

Figure 61. Comparison of experimental and simulated drift times for (i) TNT and (ii) cocaine at various flow-rate conditions. Experimental ( $\boldsymbol{\Delta})$ and simulated ( $\mathbf{})$ data. 175 
Figure 62. (i) Experimental and (ii) simulated spectra of 2,4,6-TNT and 2,7-DNF with air as both carrier and drift gas.

Figure 63. Comparison of experimental versus simulated resolution between 2,4,6-TNT and 2,7-DNF in different drift gases. Experimental ( $\mathbf{\Delta})$ and simulated ( $\mathbf{a})$ data

Figure 64. Comparison of experimental versus simulated (i) peak intensity, and (ii) FWHM at various gate width timings. Experimental $(\boldsymbol{\Delta})$ and simulated $(\mathbf{\square})$ data.

Figure 65. Response of RIP at different gate width for the FIU IMS

Figure 66. Effects of electric field at source-gate region on the reactant ion's peak response and peak shape.

Figure 67. Effects of drift electric field on the reactant ion's peak response and peak shape. .... 187

Figure 68. Effects of electric field at aperture-detector region on the reactant ion's peak response and peak shape.

Figure 69. (Left) IMS response of a $1 \mu \mathrm{L}$ spike of 2 ppm 2,4-DNT, 5 ppm 2,4,6-TNT, and $100 \mathrm{ppm}$ RDX in $\mathrm{MeOH}$ solution. (Right) IMS response curve of 2,4-DNT, 2,4,6-TNT, and RDX

Figure 70. (Left) IMS response from a $1 \mu \mathrm{L}$ spike of 30 ppm MDA, 5 ppm MA, and 30 ppm $\mathrm{BE}$ in $\mathrm{MeOH}$ solution, (Right) IMS response curve of MDA, MA, and BE.

Figure 71. (Top) FIU IMS response (at $\mathrm{G}^{\wedge} 10$ ), and (Bottom) GE IMS response of a $1 \mu \mathrm{L}$ spike of 5 ppm Pentolite explosive in ACN solution.

Figure 72. (Left) FIU IMS (at $\mathrm{G}^{\wedge} 9$ ) response of a $1 \mu \mathrm{L}$ spike of $10 \mathrm{ppm}$ MDMA and Cocaine in $\mathrm{MeOH}$ solution. (Right) GE IMS response of a $1 \mu \mathrm{L}$ spike of $1 \mathrm{ppm}$ MDMA and $10 \mathrm{ppm}$ Cocaine in $\mathrm{MeOH}$ solution. 


\section{LIST OF SYMBOLS}

K

$\mathrm{K}_{0}$

$C_{0}$

$C_{f}^{\infty}$

$C_{h}^{\infty}$

$V_{f}$

$V_{h}$

$V_{s}$

$K_{f h}$

$K_{f s}$

$K_{h s}$

$n$

$N$

$\mu$

$k$

$T_{\text {eff }}$

$\Omega_{D}$ or $Q_{D}$

$\alpha$

$\mathrm{v}_{\mathrm{d}}$

E

P

$+$

-

$\mathrm{V}_{\text {ref }}$

$\mathrm{V}_{\text {offset }}$ or $\mathrm{V}_{\text {bias }}$

$\mathrm{W}_{1}$

$\mathrm{W}_{2}$

d

$\mathrm{E}_{\mathrm{g}}$

$E_{d}$

$E_{a-d}$

$\mathrm{E}_{\mathrm{s}-\mathrm{g}}$

$\mathrm{E}(\mathrm{t})$

$v \perp$

$\Delta \mathrm{K}$

$K_{p o l}$

$P_{\text {vap }}$

$t_{d}$

$\mathrm{R}^{2}$

$w_{b}$

$w_{0.5}$
Mobility coefficient

Reduced mobility coefficient

Initial concentration

Equilibrium concentration on fiber coating

Equilibrium concentration in the sample headspace

Volume of fiber coating

Sample headspace volume

Sample volume

Distribution constant between coating and sample headspace

Distribution constant between fiber coating and sample

Distribution constant between sample headspace and sample

Mass of analyte absorb/adsorb onto the fiber coating

Drift gas density

Reduced mass of the ion-neutral collision pair

Boltzmann constant

Effective temperature

Collision cross section

Correction factor

Drift velocity

Electric field

Pressure

Positive ion

Negative ion

Reference voltage at gate

Bias voltage applied on gate wires when gate close

Gate wire 1

Gate wire 2

Distance between wire 1 and wire 2

Electric field between gate wires

Electric field of drift region

Electric field between aperture grid and detector

Electric field between ion source and gate

Radio frequency electric field

Perpendicular velocity

Net perpendicular mobility

Polarization limit mobility

Vapor pressure

Drift time of ion swarm

Correlation coefficient

Peak full width at base

Peak full width at half maximum 


\section{LIST OF ABBREVIATIONS \& ACRONYMS}

\begin{tabular}{|c|c|c|c|}
\hline ANFO & Ammonia Nitrate Fuel Oil & IMS & Ion Mobility Spectrometry \\
\hline $\mathrm{BE}$ & Benzoylecgonine & LDR & Linear Dynamic Range \\
\hline BNG & Bradbury-Nielsen Gate & LOD & Limit of Detection \\
\hline CAR & Carboxen & LSD & Lysergic Acid Diethylamide \\
\hline CAS & Control Substances Act & MALDI & $\begin{array}{l}\text { Matrix-Assisted Laser } \\
\text { Desorption/Ionization }\end{array}$ \\
\hline $\mathrm{CE}$ & Capillary Electrophoresis & MA & Methamphetamine \\
\hline CMOS & $\begin{array}{l}\text { Complementary Metal-Oxide- } \\
\text { Semiconductor }\end{array}$ & $\begin{array}{l}\mathrm{MB} \\
\mathrm{MDA}\end{array}$ & $\begin{array}{l}\text { Methyl Benzoate } \\
\text { Methylenedioxyamphetamine }\end{array}$ \\
\hline CTX & Computed Tomography X-ray & MDEA & Methylenedioxyethylamphetamine \\
\hline $\mathrm{CW}$ & Carbowax & MDMA & Methylenedioxymethylamphethamine \\
\hline DBD & Dielectric Barrier Discharge & MDP2P & Methylenedioxy phenyl-2-propanone \\
\hline $\mathrm{DC}$ & Direct Current & MEMS & Microelectromechanical Systems \\
\hline DMMP & Dimethyl Methylphosphonate & MS & Mass Spectrometry \\
\hline DMNB & Dimethyl Dinitrobutane & NG & Nitroglycerin \\
\hline DMS & $\begin{array}{l}\text { Differential Mobility } \\
\text { Spectrometry }\end{array}$ & NPC & Nanoporous-Carbon \\
\hline DNF & Dinitrofluorene & NRL & Naval Research Laboratory \\
\hline DVB & Poly(divinylbenzene) & PA & Poly(acrylate) \\
\hline EDME & Ecgonidine Methyl Ester & PBX & Plastic Bonded Explosive \\
\hline EGDN & Ethylene Glycol Dinitrotoluene & PCP & Phencyclidine \\
\hline FAIMS & $\begin{array}{l}\text { Field Asymmetric Waveform-Ion } \\
\text { Mobility Spectrometry }\end{array}$ & PDMS & Poly(dimethylsiloxane) \\
\hline FI-MS & $\begin{array}{l}\text { Fiber Introduction Mass } \\
\text { Spectrometry }\end{array}$ & PETN & Pentaerythritol tetranitrate \\
\hline FIU & Florida International University & $\mathrm{ppb}$ & part per billion \\
\hline FNA & Fast Neutron Analysis & ppt & part per trillion \\
\hline FT-IMS & $\begin{array}{l}\text { Fourier Transform-Ion Mobility } \\
\text { Spectrometry }\end{array}$ & PTFE & Polytetrafluoroethylene \\
\hline FWHM & Full Width Half Maximum & RDX & Research Department composition X \\
\hline GA & Genetic Algorithm & $\mathrm{RF}$ & Radio Frequency \\
\hline GC & Gas Chromatography & RIP & Reactant Ion Peak \\
\hline GE & General Electric & RSD & Relative Standard Deviation \\
\hline GHB & Gamma Hydroxy Butyrate & SAW & Surface Acoustic Wave \\
\hline HMTD & $\begin{array}{l}\text { Hexamethylene triperoxide } \\
\text { diamine }\end{array}$ & SDS & Statistical Diffusion Simulation \\
\hline HMX & High Melting eXplosive & SELDI & $\begin{array}{l}\text { Surface Enhanced Laser Desorption } \\
\text { Ionization }\end{array}$ \\
\hline HS & High Speed & SHV & Shielded High Voltage \\
\hline HV & High Voltage & $\mathrm{SPC}$ & Smart Preconcentrator \\
\hline IMAXS & $\begin{array}{l}\text { Intensity-Modulated Advanced X- } \\
\text { ray Sources }\end{array}$ & SPME & Solid Phase Microextraction \\
\hline
\end{tabular}


SS-

MIMS Introduction Mass Spectrometry

TATP Triacetone Triperoxide

THC Tetrahydrocannabinol

$\mathrm{THz} \quad$ Terahertz
TNA Thermal Neutron Analysis

TNT Trinitrotoluene

TSA Transportation Security Laboratory

US United States

UV Ultra Violet 


\section{PART I: PROJECT INTRODUCTION}

\section{CHAPTER 1: PROJECT MOTIVATION \& SIGNIFICANCE}

Despite the improvements in detection and prevention of, protection against, and response to terrorist explosive attacks, terrorist bombings continue to occur daily around the world. Some recent terrorist attacks around the world include the 2007 vehicle-borne attacks in London and Glasgow, the 2005 mass transit bombing in London, and the 2004 commuter train bombing in Madrid.[1] Additionally, the attacks by homegrown terrorists on John F. Kennedy Airport in 2007 served as a reminder of the continued threat to Americans after the 9/11 attacks. Hidden explosives pose a threat to safety and life not just in war zones and military situations, but also threaten innocent civilians who are targeted in terrorist bombings and endangered by landmines in post-conflict regions.[2]

While terrorist explosive attacks are considered as immediate catastrophes, an economy having millions of people who are drug users or are involved in drug related crime also requires improvement in better control of drug production, transportation, and usage. According to the United Nations world drug report for 2009, illicit drug usage has increased steadily over the last decade. In 2007, about 172-250 million people (age between 16-64 years) have used drugs at least once in the past year, and production of illicit drugs remains at high level since 2004.[3]

The ultimate aim of this dissertation is to develop analytical methods and instrumentation that can assist homeland security in the protection of the world's general population by improving detection of hidden explosives and illicit drugs. 


\section{CHAPTER 2: CURRENT CHALLENGES \& FUTURE SOLUTIONS}

\subsection{Current Challenge in Detection of Explosives and Illicit Drugs}

It is well understood and accepted that there is no single "silver bullet" detection technology that is capable of detecting all potential threats under any field environment conditions. Successful detection of hidden explosives and concealed illicit drugs requires complementary detection technologies, with each individual system designed to undertake a specific assignments such as the use of bulk detection systems to target clean samples with no trace, and trace detection systems to target samples that are well masked. All the same, each system must be designed to perform well under the environment or conditions in which it is to be deployed. For instance, trace detection systems must be effective at collecting samples and must be programmed to detect the true analytes that are actually available under field conditions.

Currently, ion mobility spectrometry (IMS) is one of the most common analytical trace detection systems deployed in the field for detection of explosives and drugs. There are approximately 15,000 analyzers making over 10 million analyses each day worldwide. IMS analyzers are available as desktop instruments for samples introduced through a particle swipe, while portal and handheld instruments exist for sampling headspace air for particles of explosives and drugs. The challenges for IMS air detectors are the collection and detection of low vapor pressure explosives and drugs. To date, IMS analyzers are programmed to detect the energetic compounds of explosives or the active constituents of illicit drugs. Some of these compounds have extremely low vapor pressure, and hence are not readily available in gaseous form to be collected by the air sampling mode. Therefore it is essential to design or program IMS vapor detectors to be capable of detecting what is actually available in the headspace air that can serve as signature compounds for detection of explosives and drugs. 


\subsection{Future Solutions: Research Hypotheses}

Vapor detection of low vapor pressure explosives and illicit drugs by IMS detector can be improved if the IMS detector is optimized to detect the volatile constituents that are emitted from explosive or drug samples. This dissertation hypothesizes:

I. Volatile compounds emitted from explosives; Detasheet, Semtex H and Composition 4 (C-4), and illicit drugs; Cocaine, MDMA, and marijuana can serve as signature compounds for headspace detection by SPME-IMS.

II. IMS operating conditions can be optimized to detect the signature compounds of interest by varying the tube temperature, gas flow rates, reactant gas, electrical field strength, etc.

III. IMS instrumental design would be well assisted with a computer modeling and simulation methodology.

IV. The availability of an in-house design IMS instrument would provide flexibility to changing the instrumental conditions for possible detection of new compounds of interest. 


\section{CHAPTER 3: RESEARCH APPROACH \& PROJECT DELIVERABLES}

In this chapter I describe the research strategies used to produce the desirable results in a timely fashion.

\subsection{Targeting the Volatile Signature Compounds of Drugs and Explosives}

Current detection technologies including IMS are programmed to target the active components of drugs and the energetic materials of explosives, which are mostly non-volatiles and hence not effective for the headspace sampling. This dissertation adopts a research approach to detect the presence of drugs and explosives based on targeting the non-active, non-energetic materials, volatile signature compounds emitted from a bulk sample.

\subsection{Systematic Optimization for Method Development}

The new compounds of interest (the target volatile signature compounds) are not typically detected at manufacturer's IMS settings because product ions are only formed under the right conditions. Hence, instrumental conditions must be optimized. An IMS instrument has a number of settings/parameters that can be varied, and therefore has a large number of possible configurations which are difficult and time consuming to evaluate exhaustively. Although optimization of instrumental conditions can be conducted by varying one parameter at a time or using a random search approach until a response is achieved, such an approach is not recognized as effective and reliable. Therefore, this dissertation develops a methodical and objective method of optimization to detect and enhance the IMS response for a list of volatile signature compounds from illicit drugs and explosives. The selected optimization method, using a genetic algorithm (GA), lends itself well to this class of multivariable optimization problem. The optimization procedure developed can be used as a tool by the IMS community for finding the optimal operating conditions of an IMS system for new compounds of interest effectively and reliably. 
While optimization can be performed on a commercial IMS instrument and achieves acceptable results, it is expected that an in-house design instrument would provide more flexibility to vary parameters and operating conditions. Therefore, this dissertation research is also dedicated to the design and fabrication of an in-house IMS for future IMS projects in the Almirall laboratory. An in-house IMS would allow for the adjustment of the instrument parameters such as the field strength, the ion gate, ionization source, etc. that would otherwise be fixed on a commercial instrument.

\subsection{Modeling and Simulation for Instrumental Design}

Before the advent of computer simulation software, scientific instruments were designed and optimized using a trial-and-error approach. Simulation using computer models has become increasingly important in the design of scientific instruments because it permits an instrument to be designed and optimized prior to expending effort and resources on physical fabrication. This dissertation is dedicated to the development of a simulation methodology for IMS instrumental design by incorporating SIMION ion optics simulation software together with a statistical diffusion simulation (SDS) modified user program to predict the IMS performance. The simulation method developed can be used as a tool by the IMS community during the design and fabrication of IMS instruments. 


\subsection{Project Goals}

Below is the list of the project's goals for this dissertation:

I The "Genetic Algorithms" programming code for systematic optimization of Ion Mobility Spectrometers to detect of new compounds of interest. Headspace SPME-IMS detection methodology for low vapor pressure plastic
explosives, Detasheet, Semtex H, and Composition-4 (C-4).

III Headspace SPME-IMS detection methodology for low vapor pressure illicit drugs, Cocaine, MDMA, and Marijuana.

An in-house design IMS instrument fabricated with full computer control and flexibility for future IMS research projects.

\section{SIMION/Statistical Diffusion Simulation method for modeling ions' behavio} inside IMS instruments at atmospheric pressure in presence of collision gas. 


\section{PART II: BACKGROUND}

\section{CHAPTER 4: THE CHEMISTRY AND DETECTION OF HIDDEN EXPLOSIVES}

CONTENT

PAGE

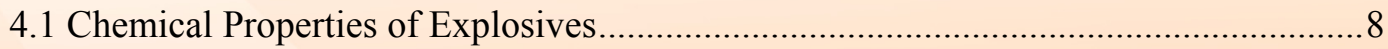

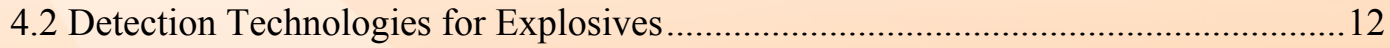

4.3 Effects of Chemical and Physical Properties of Explosives on Detection ..................20

4.4 Trace detection challenges

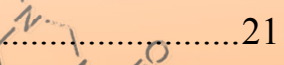

4.5 Detection of Taggants and Volatile Chemical Components of Exxpłósives................21

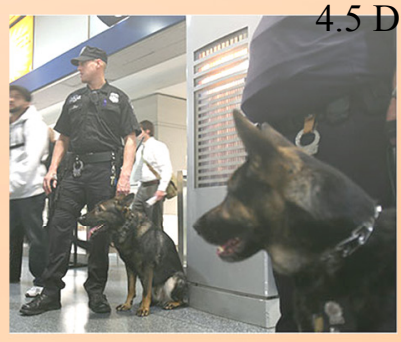

"̈
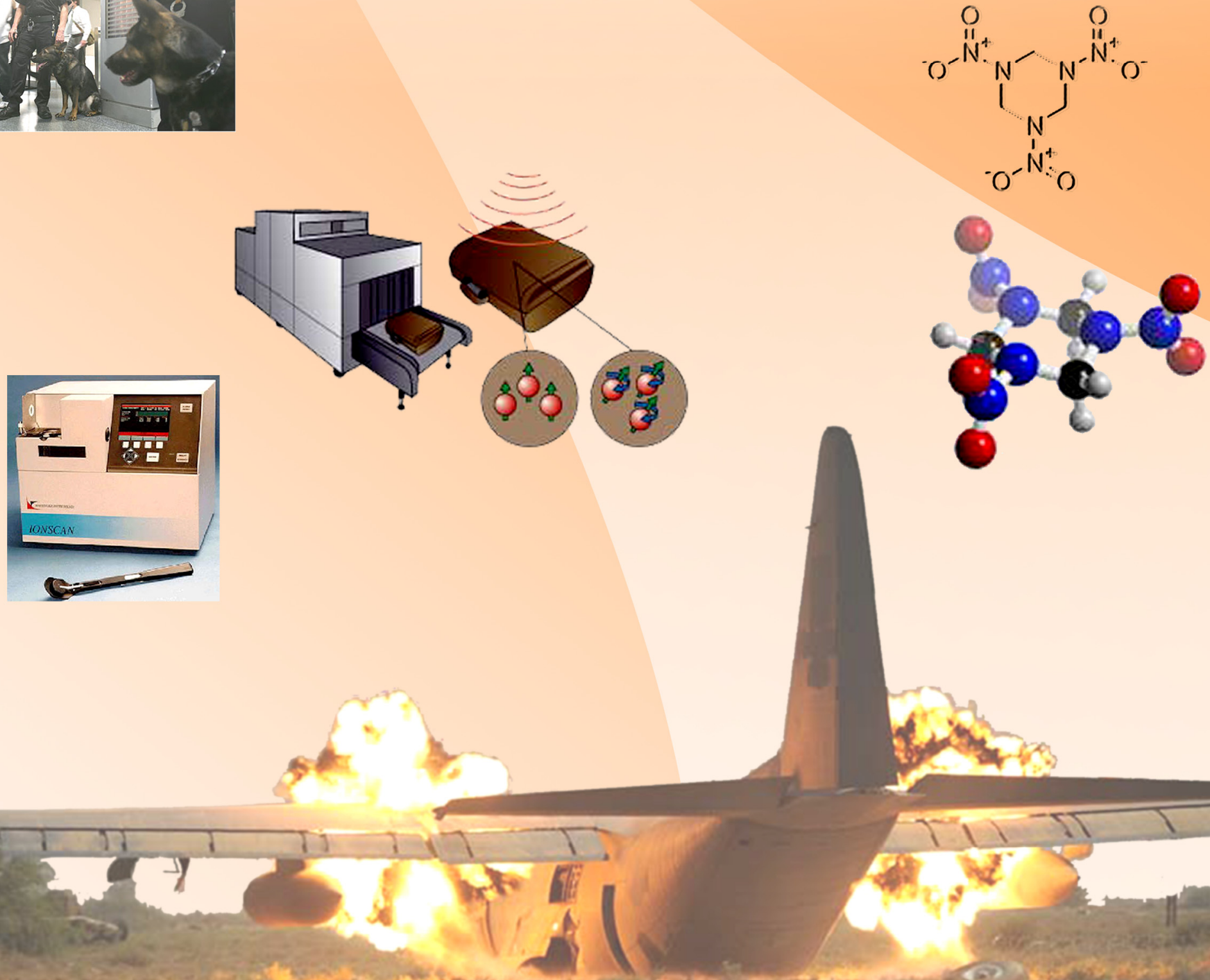
There are many contexts in which to search for and detect hidden explosives, as well as there being a great variety of explosives used in terrorist attacks and weapons of war. This dissertation focuses on the detection of plastic explosives, Detasheet, Semtex H, and Composition C-4 using ion mobility spectrometry.

\subsection{Chemical Properties of Explosives}

A brief background on the chemistry of explosives is summarized in the next few sections in the order illustrated in Figure 1.

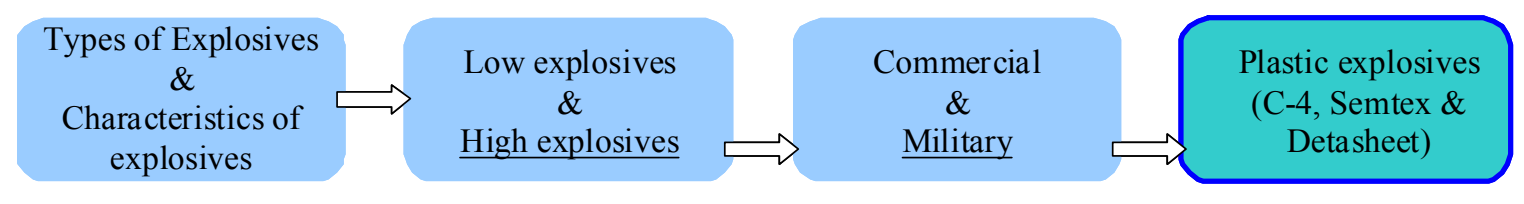

Figure 1. Content flowchart on background of explosives.

An explosive material or an energetic material is a material that is either chemically or energetically unstable, which can lead to an explosion under the right conditions. An explosion can cause a sudden expansion and decomposition of the material, producing a tremendous amount of heat and large changes in pressure, accompanied by a flash and loud noise. Explosives are classified into two groups, low explosives and high explosives, according to their rates of decomposition. Low explosives undergo decomposition at rate of less than $3000 \mathrm{~m} \cdot \mathrm{sec}^{-1}$, termed deflagration, whereas high explosives undergo detonation, in which the rate varies between 3000 to $9000 \mathrm{~m} \cdot \mathrm{sec}^{-1}$. Table 1 presents a list of low and high explosives and their chemical structures.[4] 
Table 1. Composition of low explosives and structure of high explosives.

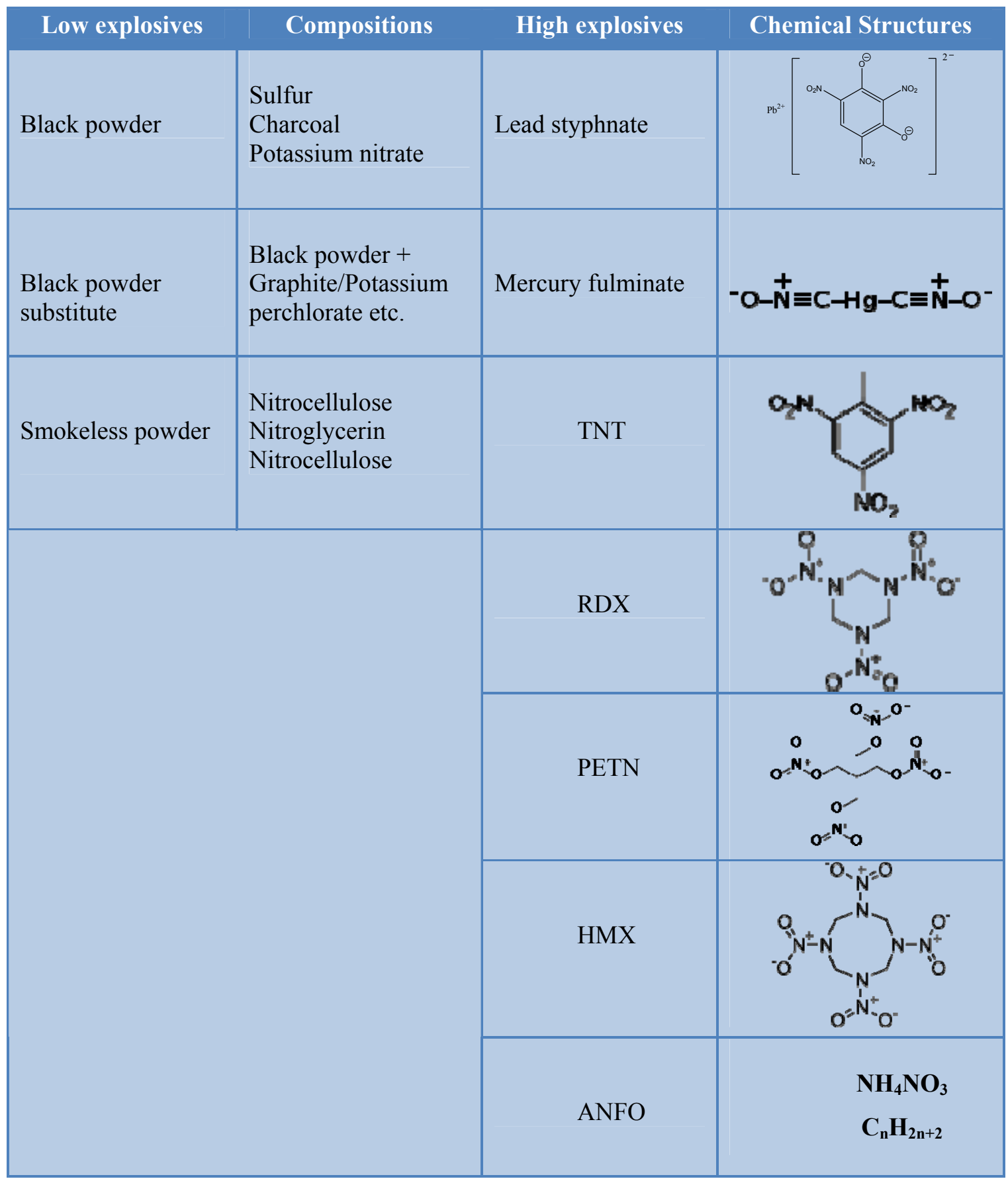

Explosives or explosive mixtures can be considered to have three essential components;

1) fuel such as carbon, hydrogen, sulphur etc.; 2) oxidizer such as nitrates perchlorides; and 3)

sensitizer which can be in chemical form or physical form.[4] Low explosives compose of a 
mixture of a fuel and an oxidant. High explosives usually chemically pure and contain both the fuel and the oxidizer components within their molecular form. High explosives can be further subdivided into two classes based on their sensitivity to initiation; primary and secondary explosives. Table 2 shows examples of high explosives separated into primary and secondary explosives. Primary explosives have lower detonation energy but are extremely sensitive to heat, friction and shock. Secondary explosives, in contrast, are relatively insensitive, but release a greater amount of energy when detonated.[4]

Table 2. List of primary and secondary explosives.[4]

\begin{tabular}{|l|c|}
\hline \multicolumn{1}{|c|}{ Primary explosives } & Secondary explosives \\
\hline Mercury fulminate & PETN \\
\hline Lead styphnate & RDX \\
\hline Lead azide & HMX \\
\hline Nitroglycerin & TNT \\
\hline Acetone peroxide & \\
\hline
\end{tabular}

In order to create an explosion, a sequence of events must occur, termed the explosive train or firing train. During the explosion process, the detonation zone or shock zone propagates at high velocity through the un-reacted explosive zone in front. Behind the shock zone is the chemical zone where the original explosive is converted into reaction products, leading to the slower moving zone as shown in Figure 2.[4]

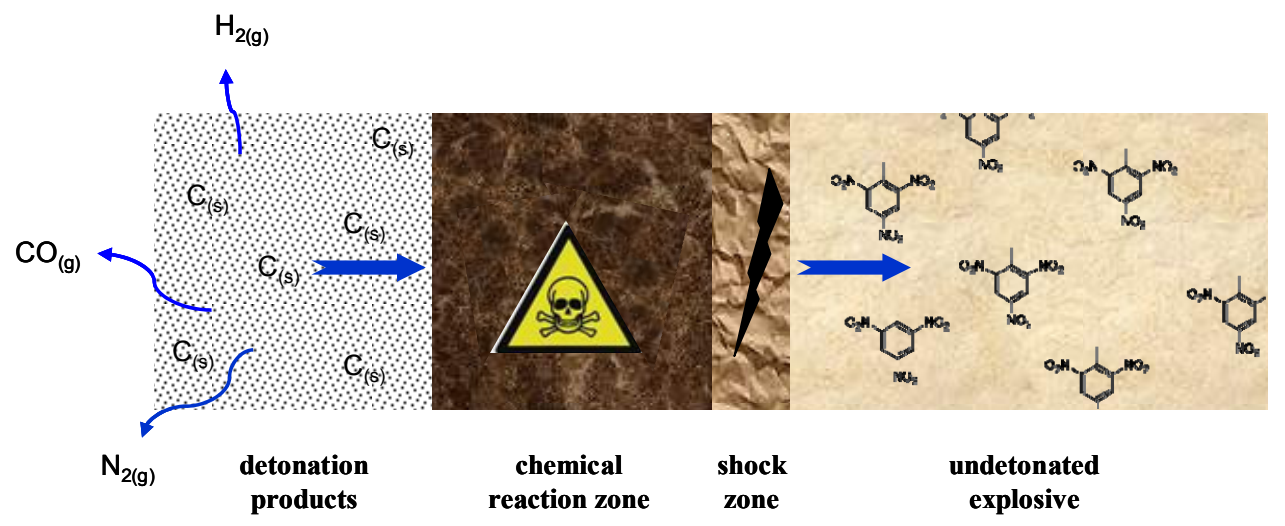

Figure 2. Detonation process of TNT explosive. 


\subsubsection{Commercial vs. Military explosives}

Commercial explosives such as dynamite, slurry explosives, and emulsion explosives are mostly used for mining and construction applications. While military explosives are commonly used in shell filling, bursting charges and demolition charges, such as black powder and smokeless powders, as well as 2,4,6-trinitrotoluene, PETN, RDX and HMX which had became common in worldwide military forces. Most of these explosive compounds can be combined and/or mixed with non-explosive additives to produce an explosive mixture with specific properties. Some examples are Composition B, Cyclotol, Torpex 2, and (C-4).[4]

\subsubsection{Plastic explosives properties}

Plastic-Bonded Explosives (PBX) or plastic explosives are commonly used in the military. Plastic explosives are malleable, have high mechanical strength and high detonation velocity, are very stable, and are insensitive to physical shocks and high temperatures. Such characteristics make them not only ideal for military demolition usage but also for terrorist bombings since they can be easily shaped for concealment purposes. Plastic explosives are mixtures that contain a range of formulations combined with a plasticizer. The most common energetic materials used are RDX, PETN, and HMX.[4] The three best known plastic explosives are C-4, Semtex, and Detasheet, and their compositions are listed in Table 3.

Table 3. Chemical composition of plastic explosives, C-4, Detasheet, and Semtex A/H.

\begin{tabular}{|l|l|l|l|}
\hline \multicolumn{1}{|c|}{ Component } & \multicolumn{1}{c|}{ Composition C-4 } & \multicolumn{1}{c|}{ Detasheet } & \multicolumn{1}{c|}{ Semtex A/H } \\
\hline Main explosive & RDX & PETN & PETN / PETN \& RDX \\
\hline Plasticizer & $\begin{array}{l}\text { dioctyl sebacate } \\
\text { diotyl adipate }\end{array}$ & & $\begin{array}{l}\text { di-n-octyl phthalate, tri-n- } \\
\text { butyl citrate }\end{array}$ \\
\hline Binder & Polyisobutylene & Plasticizer, binder, \\
Other & motor oil & $\begin{array}{l}\text { Antioxidant: N-phenyl-2- } \\
\text { naphthylamine } \\
\text { Dye: Sudan IV / Sudan I }\end{array}$ \\
\hline Taggant & DMNB & DMNB & EGDN or DMNB \\
\hline
\end{tabular}




\subsection{Detection Technologies for Explosives}

A brief background on different explosive detection technologies is summarized in the upcoming sections in the order illustrated in Figure 3.

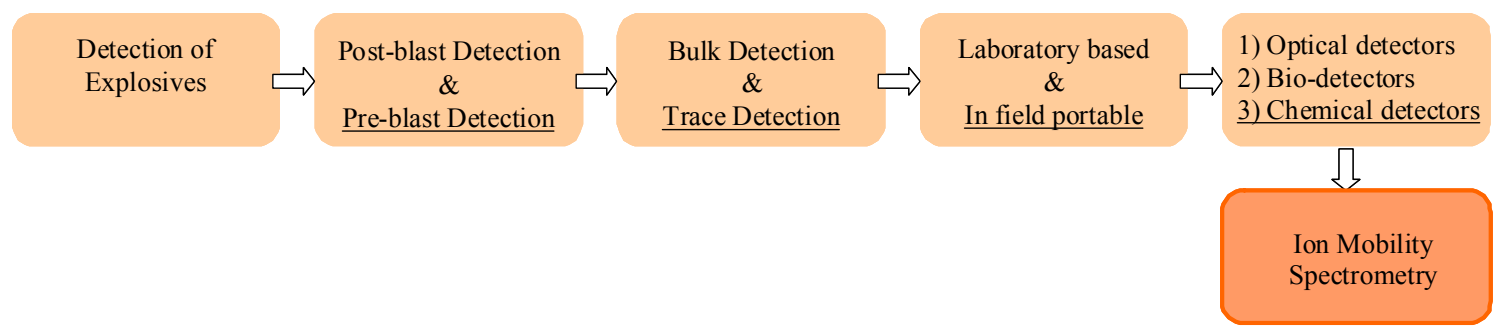

Figure 3. Content flow chart of background on explosive detection technologies.

Technologies for the detection of hidden explosives can be categorized into two groups: 1) bulk detection and 2) trace detection. The purpose of employing bulk detection technologies is to detect the main mass of explosives hidden inside concealing containers. Trace detection technologies serve the purpose of detecting small quantities of explosives that are present on or above the surface of containers as a result of contamination, or vapors penetrating from the bulk explosives.[4] Trace detection technologies are the main focus of this dissertation, however current bulk detection technologies are described briefly.

\subsubsection{Bulk Detection Technologies}

Bulk detection technologies include a broad range X-ray technology such as energy Xray, automated X-ray, and CXT (computed tomography), nuclear based technology such as thermal neutron activation, and more recently millimeter wave technology.

Detection devices based on X-ray technology were one of the first broadly employed security systems and are still in common use today. The basis of X-ray detection is a beam which, 
when it passes through different densities, reveals different images on a screen.[4, 5] Improved X-ray detectors can detect nonmetal explosives using low energy X-rays. Materials can then be classified into metals, nonmetals and intermediates, which are shown with different artificial colors on the screen and are interpreted by inspectors.. Another type of explosives detector that is in the family of X-ray devices is the CTX (computed tomography) explosive detection device which uses sophisticated image processing software to automatically screen checked baggage for explosives.[4] As of 2002, approximately 150 out of 161 explosives scanners installed by the Federal Aviation Administration (FAA) were CTX systems. Improvements to CTX technology introduced in later models such as the CTX-5000, CTX-5000 SP, CTX-5500DS, CTX-2500, CTX-9000, have allowed scanners to achieve throughput up of to 542 bags per hour.[6]

Neutron activation analysis has also been adopted, where explosives are detected by radiation of thermal neutrons and fast neutrons. The nitrogen content in explosives produces gamma rays after being radiated with thermal neutrons. Interferences induced by nitrogen from many other non-explosive materials often cause false positive alarms. Fortunately, other elements present in explosives such as carbon, hydrogen and oxygen also release powerful gamma-rays when being radiated with fast neutrons. This characteristic allows explosives to be clearly distinguished from other nitrogen interferences according to their existing nitrogen, carbon, oxygen, and hydrogen ratios.[5] However, peroxide explosives (a more recent threat) do not contain nitrogen, and these detectors are ineffective at detecting their presence. Moreover, neutrons penetrate deeply into materials, and neutron-based technologies face many problems in satisfying safety requirements and gaining public acceptance.

The most recently introduced security checkpoint technology is the millimeter wave scanner. As of April 2009, the Transportation Security Administration (TSA) has installed 
millimeter wave scanners at 19 US airports for passenger imaging.[7] In a matter of seconds, this technology can produce image that exposes weapons, explosives and other threat items concealed under layers of clothing without physical contact. The technology works by producing beams of radio frequency $(\mathrm{RF})$ energy in the millimeter wave spectrum. The beams are projected over the body's surface at high speed from two antennas simultaneously as they rotate around the body. The RF energy reflected back from the body or other objects on the body is used to construct a three-dimensional image.[7] When the above technologies are used in conjunction with trace detection technology at airports and security checkpoints, dramatic improvements can be achieved in both security and efficiency.

\subsubsection{Trace Detection Technologies}

Trace detection technology aims to target minute amount of explosive particles that are the result of contamination on the outer surfaces, or explosive vapors in the surrounding air above the explosive's container. Trace detection technologies can be in the form of laboratory based or field based instruments. Laboratory based instruments such as gas chromatography (GC), capillary electrophoresis (CE), mass spectrometry (MS), etc. are commonly used for examining post-blast evidence for explosive residues. Field detectors are mostly used to detect pre-blast explosives at security checkpoints. Field detectors can be separated into three categories, 1) biological detectors, 2) chemical detectors, and 3) optical detectors.

The most commonly used bio-detectors are canines; dogs that have been trained to detect explosives. They were the earliest trace explosives detection systems, and have been used by law enforcement agencies since the early 1970s.[4] By 2007, the number of canine teams employed at airports and mass transit systems had grown to 500 (see Figure 4). The 9/11 Commission Act requires the Secretary of Homeland Security to increase the number of explosives detection 
canine teams certified by TSA for transportation-related security by up to 200 teams annually by the end of 2010.[8]

\section{Background NEDCTP Program Growth, 2000 - 2007}

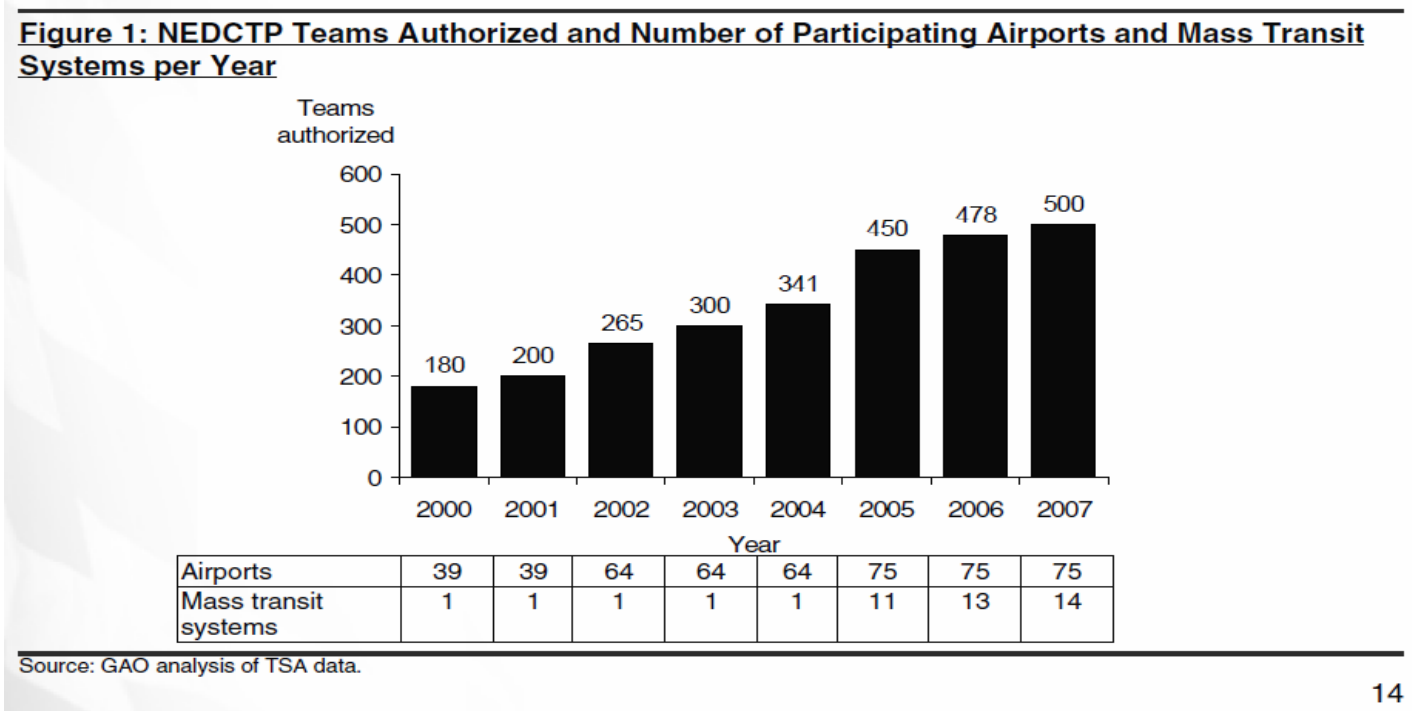

Figure 4. Number of canine teams utilized at airports and mass transit systems (2000-2007).[8]

The success of canines in detection of explosives at security checkpoints as well as in landmines removal encouraged researchers to develop explosives vapor sensors systems (electronic noses), which aim to mimic the detection and pattern recognition abilities of canines. Field mice have been successfully used in China for detection of explosives, though this approach has not been widely adopted because inspectors are not accustomed to using mice.[5]

Chemical sensor systems are based on the concept of using sensor arrays rather than individual sensors. Using arrays results in a high sensitivity to a wider range of analytes, improved selectivity because of simultaneous multi-component analysis, and permit the use of pattern recognition algorithms. By analogy with olfactory organs, which are comprised of 
multiple receptors and neural pattern recognition, sensor arrays for gas phase detection are sometimes dubbed electronic noses.[9]

There has been rapid development of polymer based sensors for the detection of nitroaromatic explosives. These detection systems rely on a range of transduction schemes including electronic and molecular interactions between the sensor polymers and the analyte. In recent years, research has explored the surface acoustic wave (SAW), fluorescent quenching, and microelectromechanical systems (MEMS) sensors. The SAW detection system recognizes the presence of nitroaromatic explosives by monitoring the shift in frequency of the piezoelectric substrate as the result of the deposition of the target analyte onto the substrate surface. Different types of polymer substrates, both organic (cyclodextrins as embedded host cavities)[10] and inorganic (siloxane polymers), have been explored and shown to have sensitivity in the level of ppb to ppt.[9-11] Fluorescence quenching of conjugated polymers is another detection method for nitroaromatic compounds.[11] The presence of nitroaromatics, an electron deficient analyte, causes electron-transfer quenching with a fluorescent polymer. The quenching process is measured as a shift in emission wavelength. Detection limits of ppb and even down to sub-ppt have been reported for conjugated fluorescent inorganic and organic polymers.[12] Fluorescent polymer sensors are by far the most sensitive detectors currently used for field detection of explosives. The Swager research group recently used conjugated polymers (those with lower excited-state oxidation potentials) for the detection of less powerful electron accepting analytes, such as in the case of taggant DMNB in plastic explosives.[13]

Micro-cantilever sensors based on piezoresistivity are a non-optical detection method. Detection is based on the bending of a micro-cantilever as a result of the interactions of the target analyte with the coated surface of the micro-cantilever. A single coating based on hydrogen 
bonding has been shown to have little selectivity. In order to achieve high sensitivity, research is being conducted to incorporate an array of the micro-cantilevers with different coatings. Unlike SAW devices, these are true microelectromechanical system (MEMS) which allow hundreds on sensors to be arrayed on a $1 \mathrm{~cm}^{2}$ chip; improving selectivity and sensitivity without increasing space. Low ppt detection limits have been reported for RDX and PETN with the SniffEx.[14, 15] Unlike the SAW or optical measuring systems for fluorescing polymers, microcantilever systems do not require frequency measuring equipment. Organic polymers have also been used to construct resistive sensors, in which a non-conducting organic polymer is coated on carbon black particles, and then deposited across metallic leads. The resistance on the leads changes as the result of the swelling of the polymer, caused by the absorption of the analyte molecules into the polymer layer. Each polymer has a different response to a target analyte depending on the partition coefficient of the analyte. Detection limits in low ppb level are observed for DNT vapor.[12] Figure 5 illustrates the limits of detection of the above mentioned electronic nose technologies in comparison to canines and single-configuration detectors such as IMS and MS.[16]

At present, canines and ion mobility spectrometry are still the most common chemical trace explosive detection systems employed at security checkpoints,[17-19] but each has notable drawbacks. Canines are generally reliable but can only work limited hours, they are labor intensive to train and maintain properly, and are subject to handler bias.[20] The actual reliability of canine detection teams is determined by the maintenance system used by the individual handlers or agencies employing them.[20, 21] IMS analyzers are preferred over other analytical instruments mostly because of their portability, sensitivity and rapid analysis time. IMS analyzers stationed at checkpoints are particulate and vapor detectors that require swiping or high volume air sampling at or close to the surface of the suspected area, respectively.[17] In order to dislodge 
low vapor explosive particulates from a surface, large amounts of air must be sampled in order to obtain enough analytes for detection. $[17,22]$ Because IMS is the detector used for this dissertation research, the theory of the technology itself merits its own chapter and is presented in Chapter 7.

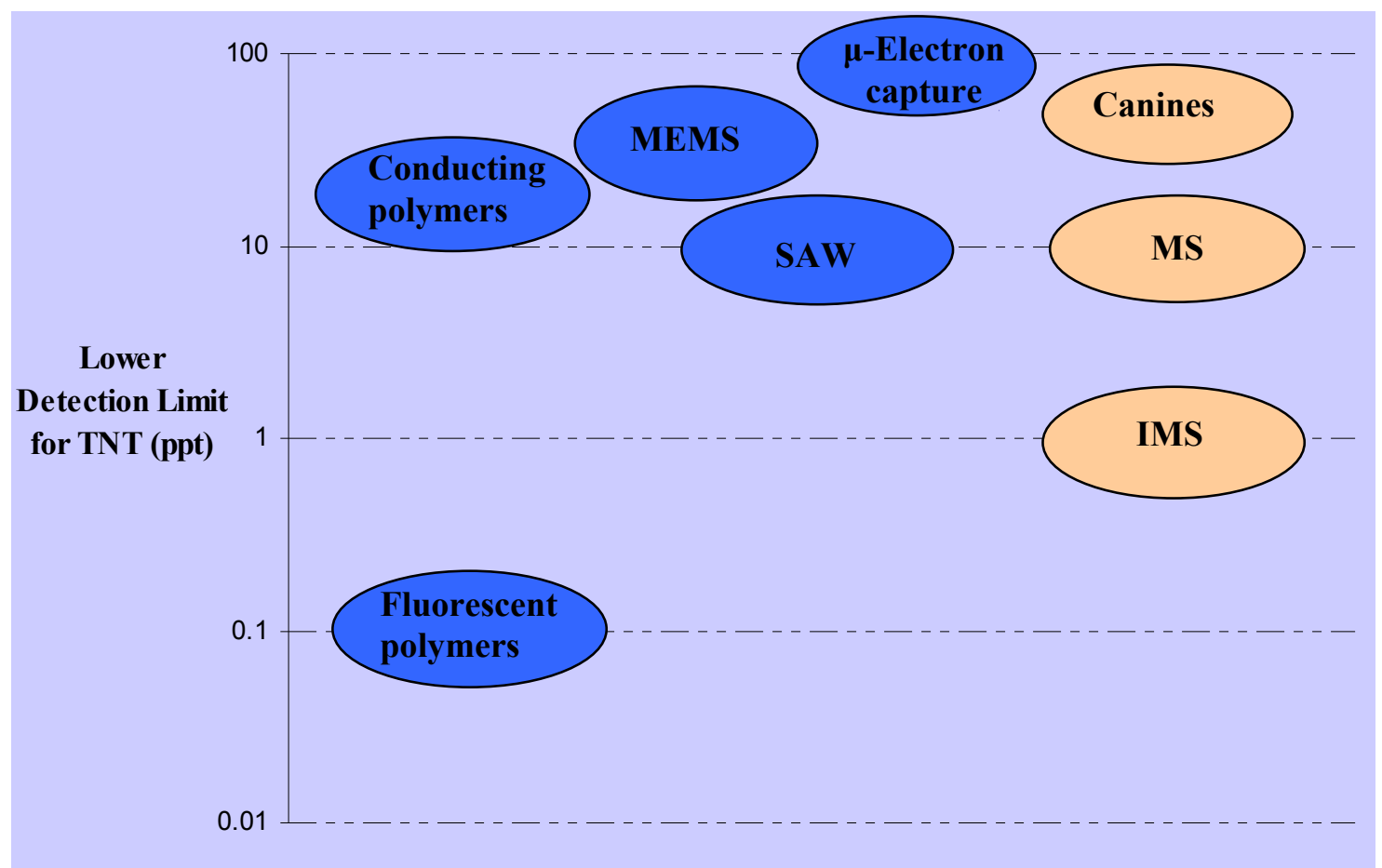

Figure 5. Detection limits of trace explosive detection systems compared to IMS detector for TNT model compound.

\subsection{Effects of Chemical and Physical Properties of Explosives on Detection}

The capability of an analytical technique to sample and detect explosives or related compounds is greatly affected by five common characteristics of explosives; 1) electronegativity, 2) adsorptivity, 3) thermal stability, 4) frangibility, and 5) vapor pressure. Various analytical techniques focus on different sets of these characteristics depending on the targeted explosives and their particular characteristics. 
Firstly, the electronegativity property of nitro and nitramine in organic explosives makes them excellent candidates for negative ionization methods, such as electron capture, because these functional groups exhibit a strong affinity for electrons. This characteristic of explosives increases the detector selectivity since most environmental contaminants are hydrocarbons that are not readily ionized to produce negative ions. Secondly, for explosives that are highly polar, they are easily adsorbed onto surfaces such as steel, wood, glass, and Teflon ${ }^{\circledR}$. In order to avoid adsorption, a detector can be maintained at high temperature, or be treated to deactivate adsorption sites. Thirdly, the thermal stability of the explosive dictates how an explosive can be introduced into a detector. Sample introduction performed by thermal vaporization must be done cautiously to prevent degradation of the analyte since explosives are by their nature thermally unstable. If molecular ions are desired, temperature must be controlled or other sample introduction techniques should be sought. Fourthly, chemical bonds of explosives are vulnerable to breakage; even a small amount of energy can rupture the bonds and cause fragmentation of the molecular ion. Therefore, soft ionization methods are usually employed when looking for molecular ions. Lastly, vapor pressure is one of the crucial properties that greatly affect the sampling capability of a technique, especially when the explosives must be in gaseous form to allow for sample collection and ionization.[23]

\subsection{Trace detection challenges}

The major challenge in trace detection technologies is the ability to effectively collect samples for analysis. Detection systems that rely on surface interaction such as swiping to collect trace explosive particles limit the search area to a small location and may also require unloading of luggage, and containers. In addition, such methods are invasive, and thus not suitable for personnel search. Trace vapor detection technologies rely on sampling the air inside or surrounding suspected objects for trace levels of airborne explosive particles. This permits the 
screening process to be performed without opening and unloading articles. However, vapor detectors cannot detect explosives which are hermitically sealed. They also cannot detect explosives whose vapor pressure is very low, such as RDX, and PETN. Realizing this potential problem, in 1991, government' officials met to create the United Nation - Convention on the Marking of Plastic Explosives for the Purpose of Identification. This act requires explosives manufacturers to mark plastic explosives with trace amounts of taggant in order to assist vapor detection systems.[24] The focus of this dissertation is on the sampling and detection of the taggants and volatile components emitted from plastic explosives using SPME-IMS.

\subsection{Detection of Taggants and Volatile Chemical Components of Explosives}

Taggants are chemicals which have much higher vapor pressure than most organic explosives (Table 4). The taggant slowly evaporates from the explosive and can be detected in the atmosphere either by canines or by specialized detectors. There are four detection taggant chemicals currently in use: 2,3-dimethyl-2,3-dinitrobutane (DMNB), ethylene glycol dinitrate (EGDN), ortho-mononitrotoluene (o-MNT), and para-mononitrotoluene (p-MNT). Taggants are added at the time of manufacture and they create a homogeneous distribution in the finished product with a minimum concentration listed in Table 4 . These minimum concentrations serve as a guideline so that the emission rate of the taggant from tagged plastic explosives is sufficient for detection by the vapor detectors. For example, the emission rate of EGDN from Semtex 1A is $2.52 \times 10^{-5} \mathrm{~g} \cdot \mathrm{cm}^{-1} \cdot \mathrm{h}^{-1} \cdot[25]$ 
Table 4. The molecular structure, vapor pressure, and minimum concentration of taggants used in the marking low vapor pressure plastic explosives.

\begin{tabular}{|c|c|c|c|}
\hline Taggant & Structure & $\begin{array}{l}\text { Vapor Pressure at } \\
25^{\circ} \mathrm{C} \text { (torr)[25] }\end{array}$ & $\begin{array}{l}\text { Tag Conc. (by \% } \\
\text { mass) [24] }\end{array}$ \\
\hline DMNB & & $1.67 \times 10^{-3}$ & 0.1 \\
\hline EGDN & & 0.06 & 0.2 \\
\hline p-MNT & & 0.11 & 0.5 \\
\hline o-MNT & & 0.03 & 0.5 \\
\hline
\end{tabular}

Although most countries have participated in the convention of marking of plastic explosives since 1991, illegal manufacturing of explosives without taggants is inevitable. In addition, unmarked plastic explosives manufactured before 1991 are still available. Thus, vapor detectors must have other means of detecting the explosives lacking the taggants. Several research groups have taken different approaches to detecting low vapor explosives by targeting the volatile chemical components emitted from the explosive mixtures rather than the energetic material themselves.[26-28] Such an approach has also been implemented for the detection of low vapor pressure illicit drugs using canines as the detector.[29-32] The volatiles emitted from the explosive samples can be compounds such as impurities, solvents, by-products, degradation products, and/or raw materials. Harper et. al. reported 2-ethyl-1-hexanol, 1-butanol acetic acid ester, and 2-ethyl-1-hexanol acetic acid as the volatiles emitted from untagged Detasheet (Flex X) explosive extracted and detected by headspace SPME-GC/MS.[33] Furton et. al reported cyclohexanone, and 2-ethyl-1-hexanol as the volatiles emanating from untagged C-4 explosive extracted and detected by headspace SPME-GC/MS.[32, 34] In both studies, only one explosive sample was analyzed. In addition to the volatiles, tagged plastic explosives contain taggants such 
as DMNB, EGDN, $p$-MNT, and $o$-MNT. IMS detection of the taggants listed above has been reported in the literature, mostly as neat standards [5, 35] or from C-4 bulk explosive.[36] Literature reduce mobility $\left(\mathrm{K}_{0}\right)$ values for DMNB and EGDN range from 1.39 to 1.49 and 1.43 to $1.66\left(\mathrm{~cm}^{2} \cdot \mathrm{V}^{-1} \mathrm{~s}^{-1}\right)$, respectively.[5, 36] $\mathrm{K}_{0}$ for $p$-MNT, and $o$-MNT are 1.45 and 1.47 , respectively.[5]

This research aims to detect the taggant DMNB as well as the volatile signatures emitted from Detasheet, C-4 and Semtex H explosives under one universal IMS setting such that when an untagged explosive is encountered, IMS would be capable of detecting the presence of the explosive based on the associated target volatiles. Multiple samples of the plastic explosives were analyzed and identified by GC/MS for better generalization of the volatiles' presence. 


\section{CHAPTER 5: THE CHEMISTRY AND DETECTION OF ILLICIT DRUGS}

CONTENT

PAGE

5.1 Chemistry of Illicit Drugs and Compounds of Interest

5.2 Detection of Concealed Illicit Drugs

西

a.

$38 \frac{1}{4}$

.

00 ? 15.

xas

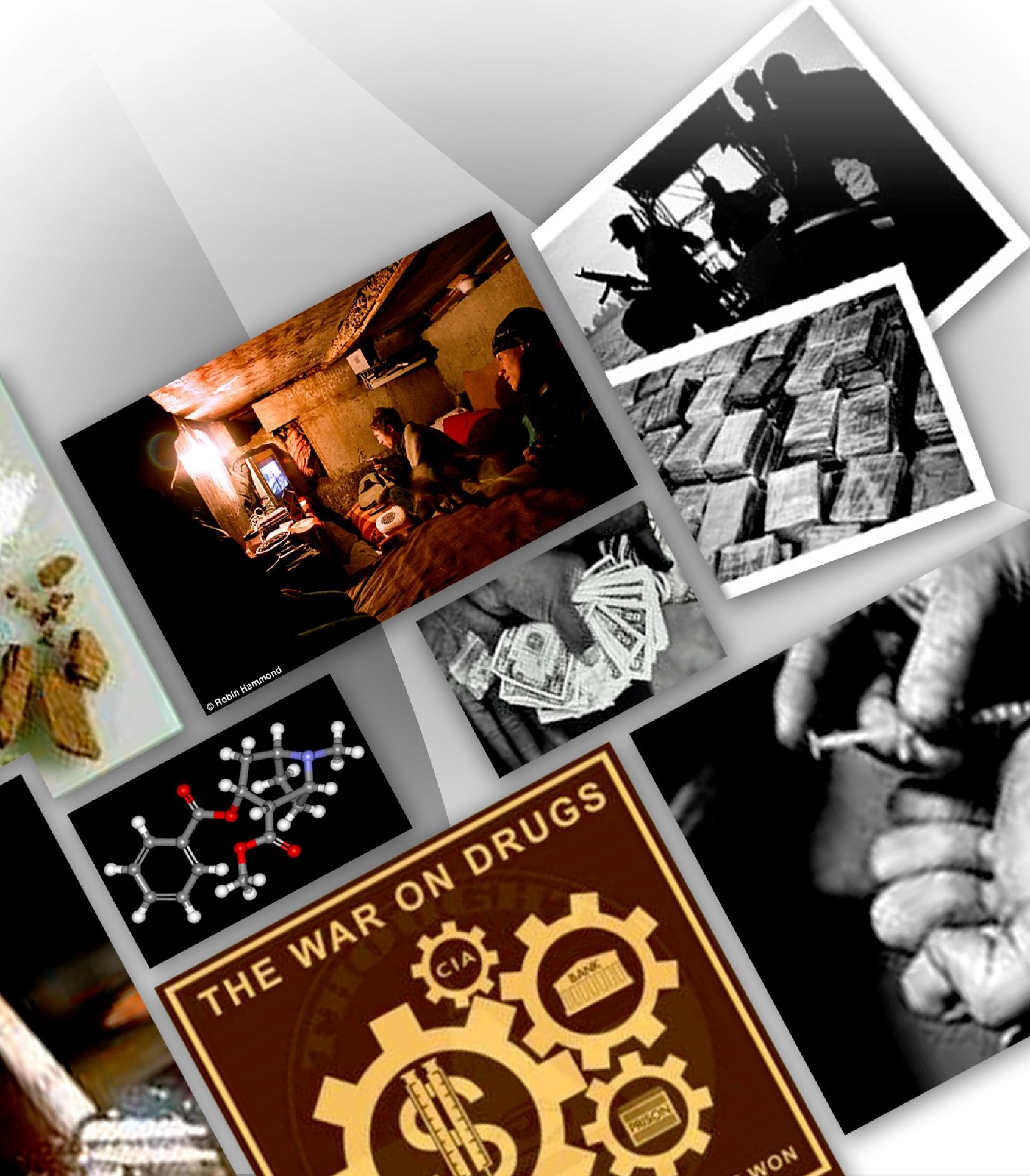


As many as 178 drugs in the United States are classified as illegal under the US Controlled Substances Act (CSA). The CSA is the federal US drug policy under which the manufacture, importation, possession, use, and distribution of certain substances is monitored and regulated. The well known illicit drugs such as heroin, cocaine, methamphetamine and marijuana are a small portion of these. Most of the remainder are chemicals used in the manufacture of other drugs, and their chemical names are thus not commonly known to the general public.[37] This dissertation focuses on the detection of marijuana, cocaine, and MDMA, three of the most commonly encountered illicit drugs in the United States. Figure 6 shows statistic findings from the Drugs Enforcement Administration's 2008 Report.[20]

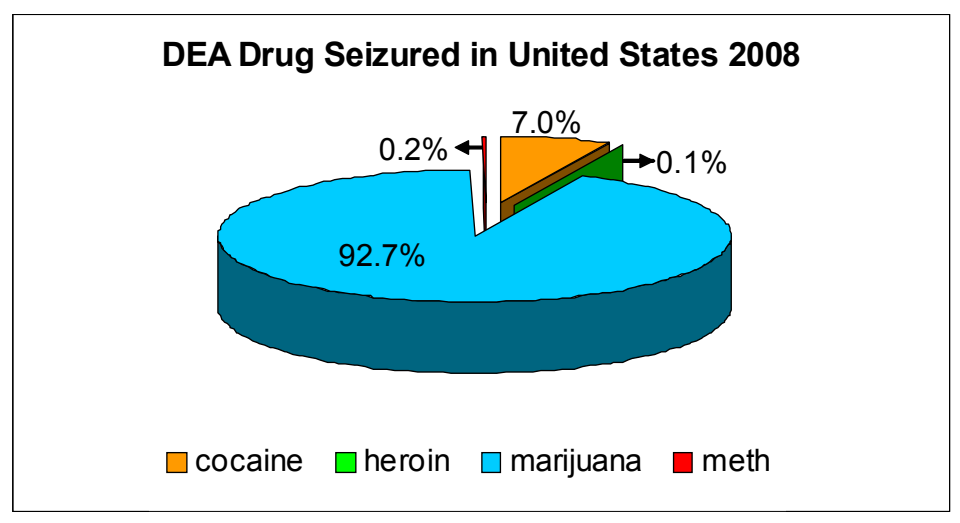

Figure 6. The DEA 2008 drugs statistics on the most common drugs seized in the US (not including hallucinogens).

\subsection{Chemistry of Illicit Drugs and Compounds of Interest}

\subsubsection{Types of Illicit Drugs}

In the US Controlled Substances Act (CSA), all illicit drugs are classified by schedule based on 1) potential for abuse and 2) medical use. Drugs can also be classified by type under four general categories; stimulants, depressants, hallucinogens, and opiates and opioids. Some 
illicit drugs may fall under more than one category. All of the illicit drugs are named psychoactive drugs because they affect the central nervous system.[37, 38]

Stimulants or psychostimulants are drugs that exhilarate the central nervous system, often referred to as "uppers". They include cocaine, amphetamine, and MDMA. These drugs can temporarily induce improvements in either mental and/or physical functions, such as wakefulness, alertness, and locomotion etc.[37] Depressants are drugs that inhibit the central nervous system, often referred to as "downers". Some of these drugs are barbiturates, GHB, and cannabis (marijuana which contains the psychoactive compound $\Delta^{9} \mathrm{THC}$ ). They temporarily diminish the function or activity of a specific part of the body or mind giving the calming, relaxing and sleeping effects.[37]Hallucinogens are drugs that cause distorted perceptions, also known as psychedelics or psychomimetics. Some well known drugs in the hallucinogens category are LSD, PCP, psilocybin, mescaline, and marijuana. There are also other drugs which are classified as hallucinogens such as MDA, which enhance self-awareness and strengthen feelings without producing sensory alterations.[37] Opiates are drugs that produced from opium, one of the oldest drugs in existence. They include heroin, morphine, and codeine. Opioids are similar to opiates in both chemical structures and biological effects. However, opioids are completely synthetic drugs and are not products of opium. Some common opioids are meperidine and fentanyl. Drugs in the opiates and opioids category account for 122 of the 178 drugs that are listed as illegal in the CSA. Their psychological effects are like depressants, which result in relaxation and joys. The physical effects of opiates and opioids depend on the individual drug, source, dose and method of intake. They slow breathing, heart rate and brain activity. Opiates and opioids depress appetite, thirst and sexual desire, but increase pain tolerance.[37] 


\subsubsection{Scheduling of Controlled Substances}

Illicit drugs are not necessarily those that are highly poisonous. They are illegal because they may result in other hazards such as addiction or inappropriate behavior to oneself and/or others. Abuse of different drugs may lead to dependency and cause physical harm to one's body or inappropriate behaviors to others.

When these drugs are used for medical purposes prescribed by licensed medical professionals, such usage is not illegal. However this kind of use is tightly controlled. The CSA federal scheduling of controlled substances is divided into 5 groups, as shown in Table 5. Schedule I contains the most strongly controlled substances, while Schedule V is the most moderately controlled. Drugs in Schedule II to V may be prescribed for medical use, with stringent restrictions for Schedule II.[38]

Table 5. Federal scheduling of controlled substances in the US.[38]

\begin{tabular}{|c|c|c|c|}
\hline Schedule & Examples & $\begin{array}{c}\text { Potential for } \\
\text { Abuse }\end{array}$ & $\begin{array}{c}\text { Accepted } \\
\text { Medical Use }\end{array}$ \\
\hline I & $\begin{array}{l}\text { LSD (Lysergic acid diethylamide) } \\
\text { MDMA }(3,4- \\
\text { methylenedioxymethylamphetamine } \\
\text { Cannabis (marijuana) } \\
\text { Heroin } \\
\text { Psilocybin }\end{array}$ & High & Not accepted \\
\hline II & $\begin{array}{l}\text { Cocaine } \\
\text { Morphine } \\
\text { Opium } \\
\text { Amphetamine } \\
\text { PCP (Phencyclidine) }\end{array}$ & High & $\begin{array}{l}\text { Accepted with } \\
\text { restriction }\end{array}$ \\
\hline III & $\begin{array}{l}\text { Lysergic acid } \\
\text { Ketamine } \\
\text { Synthetic tetrahydrocannabinol (THC) }\end{array}$ & Less than I and II & Accepted \\
\hline IV & Benzodiazepines & Low & Accepted \\
\hline V & Codeine, low dose & Lowest & Accepted \\
\hline
\end{tabular}




\subsubsection{Chemistry and Volatiles of MDMA, Cocaine, and Marijuana}

MDMA is under the stimulants category and in the amphetamines chemical class, and is a Schedule I drug. MDMA or ecstasy (a known club drug) is one of the most commonly encountered amphetamines in forensic laboratories. It can be completely synthetic, thus precursor chemicals, such as benzyl methyl ketone, and isosafrole etc. are also subjected to tight control and monitoring. There are numerous routes to synthesize the above amphetamines. More common routes are the Leuckart synthesis, nitrostyrene, and reductive amination, with benzyl methyl ketone as precursor.[37, 38] Other common pathways in which MDMA is synthesized are from precursors such as piperonal safrole.

Drugs are produced in batch processes, and hence each batch has a different impurity profile containing varying amounts of the decomposition products, by-products, and un-reacted precursors. Numerous research groups have conducted impurity profiling of MDMA tablets from seized cases. Most studies show the impurity profile obtained from liquid extraction of a powdered MDMA aliquot.[39, 40] Waddell-Smiths performed headspace SPME extraction from powdered MDMA,[41] while Lorenzo performed headspace SPME extraction from solid MDMA tablets.[21] Shown in Table 6 are the reported impurity profiles from MDMA cases studied by the above groups. Compounds reported from a headspace SPME study of MDMA tablets serve as possible target compounds for vapor detection by IMS, as in this dissertation, because of their higher vapor pressure (see Table 7) as compared to MDMA itself at $0.00 \mathrm{mmHg}$ at $25^{\circ} \mathrm{C}$.[15] 
Table 6. Impurity profiles of MDMA reported in various studies found in literature.

\begin{tabular}{|c|c|c|}
\hline Research by & Impurities & Sample studied \\
\hline Palhol et al.[39] & $\begin{array}{l}\text { MDP2P } \\
\text { Palmitic } \\
\text { stearic acid }\end{array}$ & 52 tablets/ liquid extraction \\
\hline Cheng et al.[40] & $\begin{array}{l}\text { MDP2P } \\
\text { MDP } \\
\text { MDA } \\
\text { MDB } \\
\text { Piperonal } \\
\text { Ketamine }\end{array}$ & $\begin{array}{l}80+\text { tablets from a batch of } \\
120,000 \text { tablets seized in Hong } \\
\text { Kong/ liquid extraction }\end{array}$ \\
\hline Waddell-Smith[41] & $\begin{array}{l}\text { benzeneacetamide } \\
\text { 3,4-methylenedioxy-N,N- } \\
\text { dimethylbenzylamine } \\
\text { 3,4-methylenedioxyamphetamine } \\
\text { acetate } \\
\text { caffeine } \\
\text { Piperonal (at } 70^{\circ} \mathrm{C} \text { extr.temp.) }\end{array}$ & $\begin{array}{l}\text { Powdered/ Headspace-SPME at } \\
50{ }^{\circ} \mathrm{C}\end{array}$ \\
\hline Lorenzo et al.[21] & $\begin{array}{l}\text { Benzoic acid } \\
\text { MDP2P } \\
\text { MDP } \\
\text { Methamphetamine } \\
\text { Piperonal }\end{array}$ & Tablet/ Headspace-SPME at RT. \\
\hline
\end{tabular}

Table 7. Chemical structure and vapor pressure of impurities found in headspace air above MDMA .

\begin{tabular}{|l|l|l|}
\hline \multicolumn{1}{|c|}{ Impurities } \\
$\begin{array}{l}\text { Methylenedioxy) phenyl-2- } \\
\text { propanone ) }\end{array}$ \\
\hline Isosafrole
\end{tabular}


Cocaine is a Schedule II drug under the controlled substances act, with high dependency but accepted medical use. It is both a stimulant of the central nervous system and an appetite suppressant. Cocaine is a major alkaloid produced from a natural plant called Erythroxylon coca after a crude leaf extraction, and purification process. Illicit cocaine exists in $\mathrm{HCl}$ salt form (known as "snow") and in free base form (known as "rock" or "crack"). The free base form is more volatile, thus normally used for smoking.[38] Along with cocaine, many other alkaloids based upon the tropane structure (bicyclic tertiary amine) from coca extract can be converted into cocaine. The major alkaloids include ecgonine, methylecgonine, and benzylecognine. One of the common routes of cocaine synthesis is through the hydrolysis of the alkaloids to ecgonine, followed by esterification to yield cocaine. Incomplete esterification can lead to ecgonine based impurities. The most commonly encountered impurities are ecgonine, methylecgonine, benzoylecgonine.[43] Other impurities in illicit cocaine, such as benzoic acid, ecgonidine methyl ester (EDME),[44] and trans-cinnamic acid, have also been reported.[31, 45, 46] Cocaine samples that are old or been stored under harsh conditions (high humidity) can also have decomposed to form methyl benzoate (MB) $[31,47,48]$ and EDME.[49, 50] Methyl benzoate and EDME are possible target candidates for SPME-IMS detection of cocaine because the vapor pressure of EDME and methyl benzoate is 5 and 7 orders of magnitude higher than cocaine, respectively.[51] Shown in

Table 8 is the vapor pressure of the reported impurities found in cocaine. As seen, some of these compounds have much high vapor pressure compared to cocaine itself $\left(0.37-1.2 \times 10^{-7}\right.$ torr at $\left.20^{\circ} \mathrm{C}\right)$. 
Table 8. Chemical structure and vapor pressure of impurities reported in headspace air of cocaine.

\begin{tabular}{|l|l|}
\hline Ecgonine & Impurities \\
\hline Bethyl ecgonine & Not found \\
\hline Benzoic acid & $7.5 \times 10^{-4} \mathrm{mmHg}$ at $25^{\circ} \mathrm{C}$ \\
\hline Ecgonidine methyl ester
\end{tabular}

Cannabis, commonly known as marijuana or marihuana, is cultivated from Cannabis sativa L. plant. It is intended for use as a psychoactive drug containing many different cannabinoids. The most common form of cannabis used as a drug is the natural herbal form, which consists of the dried flowers, stalks, and leaves of mature female plants. Another form of cannabis products is the resinous material that is collected from the surface of the plant, dried and pressed into blocks. Alternatively, cannabinoids can also be extracted from the herbal form or the resin to produce hash oil with increasing potency. There are numerous types of cannabinoids that are present in cannabis. The major psychoactive chemical compound in cannabis is $\Delta^{9}$ - 
tetrahydrocannabinol, commonly abbreviated as $\Delta^{9-} \mathrm{THC}$ (the chemical structure is shown in Figure 7). This compound is responsible for the pharmacological activity of cannabis. $\Delta^{9}$-THC is formed in the glandular trichomes which are typically found on the surface of the plant. The content of $\Delta^{9}$-THC is lowest in the herbal leaf $(1 \mathrm{wt} \%)$, followed by the flower $(3.5 \mathrm{wt} \%)$. Resin contains between 2-10 wt \%, while hash oil can generally be $10-30 \mathrm{wt} \%$. Other parts of cannabis plant with lower or no $\Delta^{9}$-THC content, i.e. seeds and stems, have long been used for the production of seed oil, rope and fabrics, respectively.[38]

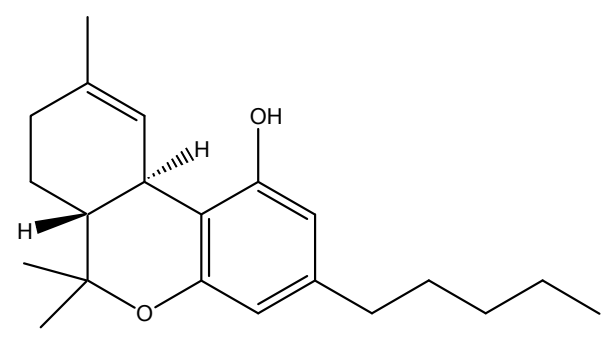

Figure 7. Chemical structure of $\Delta^{9}$-tetrahydrocannabinol.

At current date, cannabis drugs are used for recreational, religious or spiritual, purposes, but have not yet been accepted for medical use. In the United Nations 2009 World Drugs Report, it estimated in 2007 , only $11 \%$ of cannabis products (cannabis herb and resin combined) are seized $(\sim 7000$ out of 62,400$)$ million tons.[26] Cannabis herb and resin are also the most consumed drugs in the United States. $\Delta^{9}$-THC has extremely low vapor pressure, insufficient for vapor sampling. Fortunately, there are hundreds of volatile constituents of marijuana, with the following identified as the major components: limonene, $\alpha$-pinene, $\beta$-pinene, $\beta$-myrcene, and $\beta$ caryophyllene.[46, 52] Their structure and vapor pressures are listed in Table 9. 
Table 9. Chemical structure, and $\mathrm{P}_{\mathrm{v}}$ of dominant volatile constituents from marijuana.

\begin{tabular}{|l|l|l|}
\hline Lolatile constituents & \\
\hline$\beta$-pinene & & \\
\hline$\beta$-myrcene & & \\
\hline$\beta$-caryophyllene & & \\
\hline
\end{tabular}

\subsection{Detection of Concealed Illicit Drugs}

The purpose of this section is to give a brief background of the different technologies that are available and recently developed for field for detection of concealed illicit drugs. Background on the different laboratory analysis methods of illicit drugs (forensic evidence) is omitted because it is not the focus of this dissertation.

\subsubsection{Bulk Detection Technologies}

Bulk detection technologies are widely used for package surveillance and large cargo inspection since the technologies allow for screening without opening and unloading of cargo containers, etc. The current most broadly employed systems are X-ray technologies. Terahertz and neutron-based technologies have also received recent attention. These same systems are used for explosives and other contraband detection. 
X-ray technologies are widely used because the technology is well advanced and their presence is generally accepted in public areas, given that they operate below an allowable radiation footprint. High X-ray intensity is sometimes necessary to penetrate through dense cargo for inspection. The Intensity-Modulated Advanced X-ray Sources (IMAXS) system recently developed by HESCO/PTSE Inc., Schonberg Research Corporation and Rapiscan Laboratories is a variable intensity pulse source.[53] This type of source allows cargo inspection systems to achieve up to two inches greater penetration capability, while still retaining the same radiation footprint as compared the present fixed-intensity source.[53] Nevertheless, X-rays have small interaction probability with low electron density organic materials, which is sometimes the case for illicit drugs and is commonly the cases for explosives. These materials have undistinguishable X-ray absorption or incoherent scattering characteristics, thus making detection difficult. Some X-ray systems can produce sharp image which is useful for inspection by shape recognition.[54] However, detection of illicit substances cannot be dependent on shape recognition since drugs and explosives can be packed or molded into any desirable shape. Limitations of X-ray systems encouraged the development of other systems like neutron-based technologies.

There have been at least seven different technologies developed that are neutron-based. The two best known are thermal neutron analysis (TNA) and fast neutron analysis (FNA). Neutron-based methods are effective means of elemental characterization of a bulk cargo that is densely packed and hence poses a challenge to some X-ray systems. Neutrons can penetrate deeply into the package and interact uniquely with the target nuclei, giving distinguishable images of drugs and explosives against a busy background. Their superb penetration capability also raises many problems in satisfying safety requirements and gaining public acceptance. In addition, neutron-based systems screen at a much slower speed of 4 trucks per hour.[53, 54] 
Therefore, neutron-based screening systems are unlikely to replace current X-ray screening units in the near future.

Millimeter wave and Terahertz (THz) imaging are promising technologies for illicit drug detection and inspection due to their strong penetrability for clothes, paper bags, and leather or plastic luggage, without harming living tissue. THz technology provides not only images, but also fingerprint spectra that are unique to most illicit drugs. Therefore, compared to other bulk inspection techniques, $\mathrm{THz}$ technology can identify both the shape and type of illicit drugs in question. To identify samples, a component spatial pattern analysis method is employed in the data processing. The system recognizes the shape of the absorption spectra instead of the absorption peaks of detected samples, which provides increased accuracy. Lu et. al. successfully showed distinguishable absorption spectra of MDA, MDMA, heroin, morphine, acetylcodeine, and ketamine.[55] The capabilities of millimeter wave and terahertz technology were quickly appreciated by the law enforcement community, but the technology has not yet won public acceptance as a portal system due to privacy issues arising from exposed computer images of an individual.

\subsubsection{Trace Detection Technologies}

Trace detection technologies for screening illicit drugs are similar to those used for trace explosives detection systems. Most systems can be deployed to simultaneously detect both drugs and explosives, or have two separate operating modes. Vapor bio-detectors and vapor chemical detectors are most common for screening large cargo containers for illicit drugs. Particle detectors, which are better suited in airport settings with a small search surface (purse, laptop etc.), are usually set for explosives detection. Vapor detection is desirable for large container screening when opening and unloading of cargo for sampling is otherwise restricted by time 
constraints. Sampling of vapor involves drawing air out of the container through a duct leading to a sampling area where the volatiles are concentrated on a pre-concentration device and then subjected to a vapor detector (bio or chemical) for analysis. The following is a focused background summary of vapor detection technologies for the trace detection of illicit drugs. Recently, Staples et. al.[46] reported the development of a high-speed GC (un-coated) SAW sensor system. This system detects the presence of illicit drugs based on chemical profiling for the volatiles signature present, and has picogram sensitivity. The HS GC SAW unit constructs a visual vectorial image in $\mathrm{N}$-dimensional space (where $\mathrm{N}$ equals the number of sensors) of a specific vapor mixtures containing possibly tens or hundreds of different chemical species which correspond to a specific target substance. Staples's study reported the detection of marijuana and cocaine inside test cargo containers using the HS GC SAW system and a preconcentrator. Within 2 to 60 seconds sampling time and 20 seconds of analysis time, the system provided an image (component map) of marijuana and cocaine based on their volatile signatures. The HS GC SAW sensor system has great potential to become a chemical vapor detector of choice in the future due to its portability, fast analysis time, high sensitivity, and reusability. At the current research stage, HS GC SAW has a probability of detection equal or greater than $90 \%$ and false alarm rate of 1 \%.[46] Other SAW vapor sensor systems, such as SAW immunoassay also reported the detection of cocaine.[56] However, this system is not a true vapor detector because testing was performed with cocaine air samples created from a vapor generator at elevated temperature.

Similar to explosive detection, canines and ion mobility spectrometer are the two most common trace vapor detectors currently deployed in the field. Canines are known to alert to illicit drugs such as cocaine and MDMA by detecting methyl benzoate [45] and piperonal/MDP2P mixtures,[21] respectively. Furton et al. found that more canines alert to methyl benzoate than to any other volatile impurities of cocaine or pharmaceutical grade cocaine itself (no methyl 
benzoate is present).[45] Earlier canine studies conducted by Waggoner et al. on canine olfactory sensitivity to cocaine $\mathrm{HCl}$ and methyl benzoate suggested that the canine response to illicit cocaine $\mathrm{HCl}$ could be the result of multiple volatile constituents rather than methyl benzoate alone. In their study, the canine's threshold sensitivity for pure methyl benzoate was $16 \mathrm{ppb}$ compared to $0.03 \mathrm{ppb}$ (methyl benzoate concentration) from an illicit cocaine vapor sample.[31] Lorenzo et al. reported in a canine study that six out of six of the dogs alert to piperonal/MDP2P mixture or MDP2P alone, and five out of six canines alert if only piperonal was used. Sample size ranged between 4 to $30 \mathrm{mg}$.[21] Despite the numerous reports of canine detection of illicit drugs based on volatile signature compounds, commercial IMS vapor analyzers are currently only programmed to detect illicit drugs based on the parent compounds themselves. However, in most cases these are non-volatiles. Different research groups have reported the detection of ecgonidine methyl ester (EDME) in cocaine $\mathrm{HCl}$ vapor using IMS, [44, 49] but none have reported the detection of methyl benzoate by IMS. Similarly, no other research has reported detection of piperonal or MDP2P from MDMA vapor by IMS. As for vapor detection of marijuana, Kim et. al. in the early 1990's reported the detection of the monoterpenes and trans(or $\beta$ )-caryophyllene, volatile constituents of marijuana by IMS using acetone or ammonia as reactant gas.[52]

In summary, Section 5.1 covered the background on chemistry of illicit drugs MDMA, cocaine, and marijuana with strong emphasis on their volatile constituents that are results from impurities, decomposition products etc. Section 5.2.2 summarized the current trace vapor detection technologies and their target volatile signatures. This dissertation aims to optimize the operating conditions of a commercial IMS instrument to detect low vapor pressure illicit drugs, cocaine, and MDMA, marijuana $\left(\Delta^{9}-\mathrm{THC}\right)$ by targeting both the volatile signature compounds that canines have successfully detected in the past studies, as well as other known volatile constituents and impurities. 


\section{CHAPTER 6: SAMPLING AND PRECONCENTRATION DEVICES}

CONTENT

PAGE

6.1 Desired Characteristics of Preconcentration Devices...................................................38

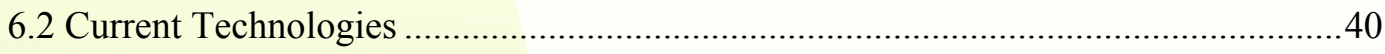

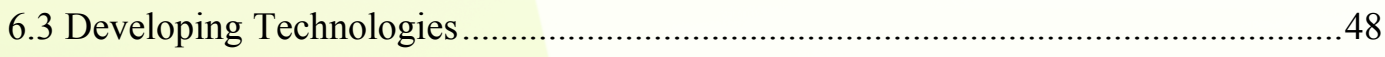
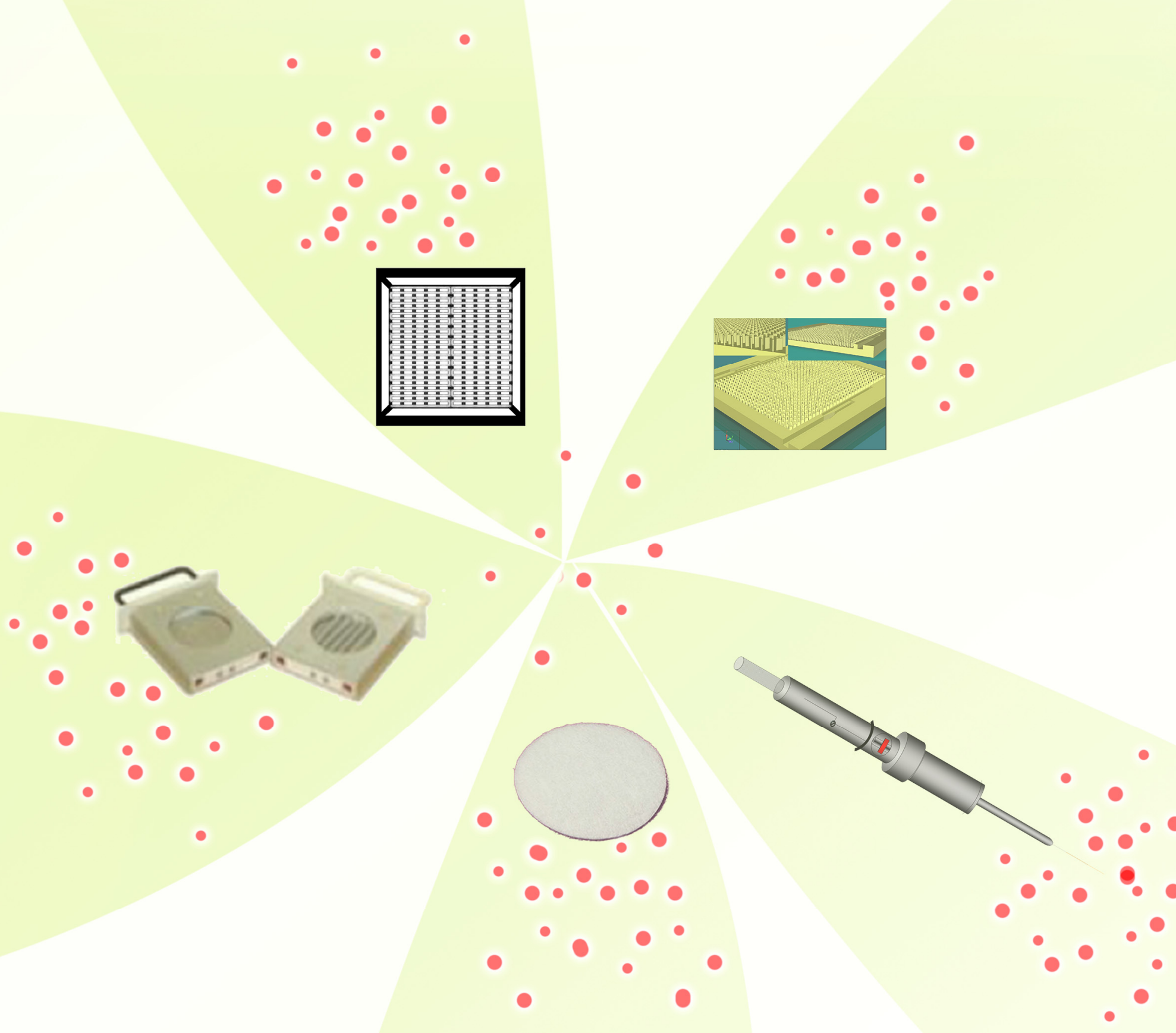
Commercial trace explosive and drug detectors have very low detection limits, between picogram to low nanogram levels.[16, 17, 57] Nevertheless, these sensitive detectors need to be accompanied by an effective front end sampling system for collection and transportation of the target analyte to have practical applications. Physical swiping is an effective means of collecting particulates that reside on surfaces since low vapor pressure explosives and drugs tend to stick to a surface for a long time. However, such a method is not suitable for screening large containers or personnel. Headspace air sampling techniques, on the other hand, are be able to collect samples in situations where air containing vapors or particulates of the target analyte can be drawn from the source for analysis. Because the space being sampled varies in size, and includes large volume containers, the headspace concentration is extremely diluted, usually much lower than the detection limit even for the most sensitive detector. Sample preconcentration is therefore highly compulsory in order to successfully detect analytes in such situations.

This chapter outlines the desired characteristics of a preconcentration device in Section 6.1. Sections 6.2 and 6.3 discuss the background of current and developing technologies respectively. Section 6.2.1 gives extra emphasis to solid phase microextraction.

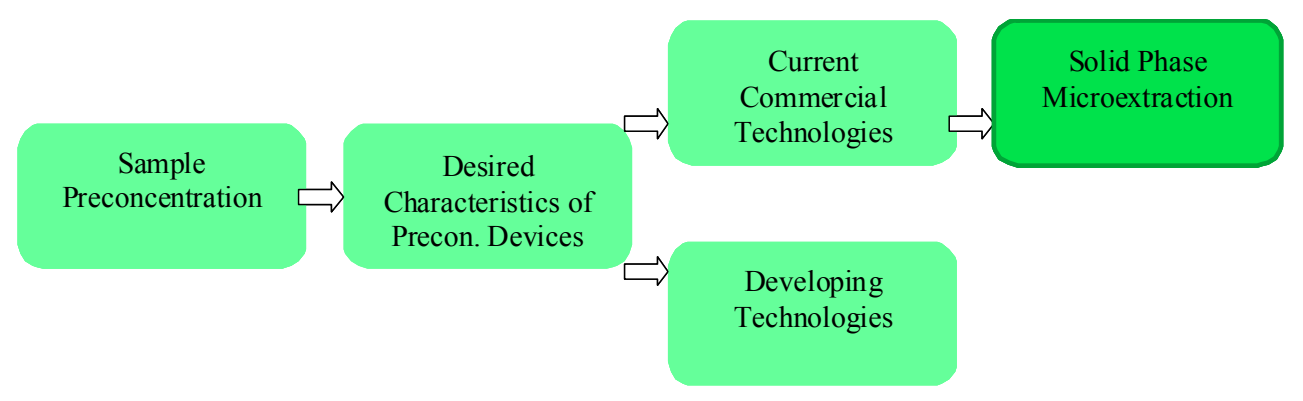

\subsection{Desired Characteristics of Preconcentration Devices}

Desirable performance characteristics of a preconcentrator utilized for dynamic air sampling include the capability to operate at high flow rates, fast thermal heating cycles 
(assuming thermal vaporization is the method for releasing the analyte), fast clearing times, high selectivity toward analytes of interest, and, most importantly, a high concentration factor.[58]

Preconcentration devices must have high mechanical strength in order to withstand high flow rates of air passing through them. This is necessary because the headspace concentration of analytes are usually minimal in large containers, which requires longer sampling time to draw large volume of air (between tens to thousands of liters) to collect enough analyte for analysis. Air flow rates required to pass through a preconcentration device varies from one technology to another. Depending on the opening area of the device, the perpendicular force can be very high and quickly deform the structure if it is not mechanically strong enough. For example, a design from Sandia National Laboratory has an inlet opening diameter of $5 \mathrm{~cm}$ that can allow air to pass through at $\sim 3000 \mathrm{~L} \cdot \mathrm{min}^{-1}$, achieving a concentration factor of $\sim 1400$.[57] With such a flow rate, a thin membrane preconcentrator design would withstand the forces associated with high flowrate.

The preconcentration device must be constructed from materials that can offer fast thermal heating and cooling cycles. As some detectors' total analysis time is on the order of seconds, the preconcentration device must quickly rise to optimum temperature in order to give a sharp desorption profile and completely release the trapped analytes into the analyzer. The preconcentrator must also cool down to sampling temperature in reasonable time in order to be ready for the next preconcentration and analysis cycle. The necessary thermal desorption temperature varies depending on the type of analytes and the preconcentrator being used, but usually ranges between $180{ }^{\circ} \mathrm{C}$ to $300{ }^{\circ} \mathrm{C}$.

Fast clearing time is desirable to ensure samples without carryover. Clearing times for current technologies usually take up to 1 minute, but can be as short as 5 seconds. 
In addition to a high concentration factor, selectivity of preconcentration devices makes them a crucial component at the front end of a detector. Preconcentration devices also act as a front line selective guard to only attract the analytes of interest for analysis, while allowing interference analytes to pass right through. This effect minimizes possible interference and competition within the detector, and improves the accuracy of the detection method. Depending on the objectives, the preconcentration device can be designed to select a specific characteristic of the analyte, i.e. molecular weight, and polarity. A well designed preconcentration technology can have a range of possible applications, given that the preconcentration substrate is specified for a particular group of analytes.

\subsection{Current Technologies}

Commonly used preconcentration methods are charcoal beds, membrane filters, or solid surfaces to trap analyte vapors. However, these preconcentrators offer little or no selectivity for explosives and are often mechanically too weak for high flow rate sampling.[59] Several preconcentrator designs with stronger mechanical properties are available for high volume air sampling, such as porous metallic filter meshes, woven wire meshes, or sheets of metallic felt.[57] The Hound (or Hound II) and the microHound preconcentrator is an example of the metal mesh technology that was developed at the Sandia National Laboratory.[57] Vapors as well as particles are collected and preconcentrated by flowing air through the device followed by thermal desorption for analysis by an IMS analyzer. Since low vapor pressure explosive molecules are sticky, they easily adsorb onto the wires of the mesh. However, high vapor pressure explosive molecules typically pass right through without sticking onto the wires, resulting in false negatives.[60, 61] 
Other preconcentrator designs with enhanced selectivity and sensitivity are those with a coated polymer phase. An example is the sorbent-coated micro-fabricated devices recently developed at the US Naval Research Laboratory (NRL). The NRL preconcentrator is based on a suspended array of micro hot plates designed using complementary metal-oxide-semiconductor (CMOS) technology and coated with a thin polymer layer. The temperature of the device can increase to $180^{\circ} \mathrm{C}$ in $40 \mathrm{~ms}$, allowing molecules to quickly be adsorbed or desorbed. The NRL preconcentrator has been used for a commercial IMS (VaporTracer 2, GE Iontrack), and is able to improve the signal intensity of TNT by an order of magnitude for a 10 second preconcentration time. $[58,62,63]$

A more mature technology based on coated polymer phase is solid phase microextraction (SPME), developed by the Pawliszyn research group in the 1990's. SPME is briefly introduced here, and further details on SPME theory are summarized in Section 6.2.1. SPME offers many advantages including fast extraction and desorption, field adaptability, high selectivity and sensitivity, and ease of use. SPME has been shown to offer many advantages for the extraction and preconcentration of volatile and semi-volatile components from the headspace of a sample.[64] SPME devices, however, suffer from limited capacity, they are easily broken or stripped, and no commercial interfaces to field detectors accept the GC inlet. By recognizing the later drawback, Perr et. al. developed an in-house design SPME interface that can be adapted to the front end of the GE Itemiser 2 IMS.[27] Although SPME is most commonly used with gas chromatography, SPME has also been used with mass spectrometer as well as in the second preconcentration stage after SS-MIMS (single-sided membrane introduction mass spectrometry). Cotte-Rodríguez et. al. showed that the SS-FI-MS (single-sided membrane fiber introduction mass spectrometry) combined technique improves sensitivity by factor of 23 over the FI-MS 
(fiber introduction mass spectrometry) alone (3.5).[59] Figure 8 shows the relative preconcentration factor of SPME devices with respect to other preconcentration devices.

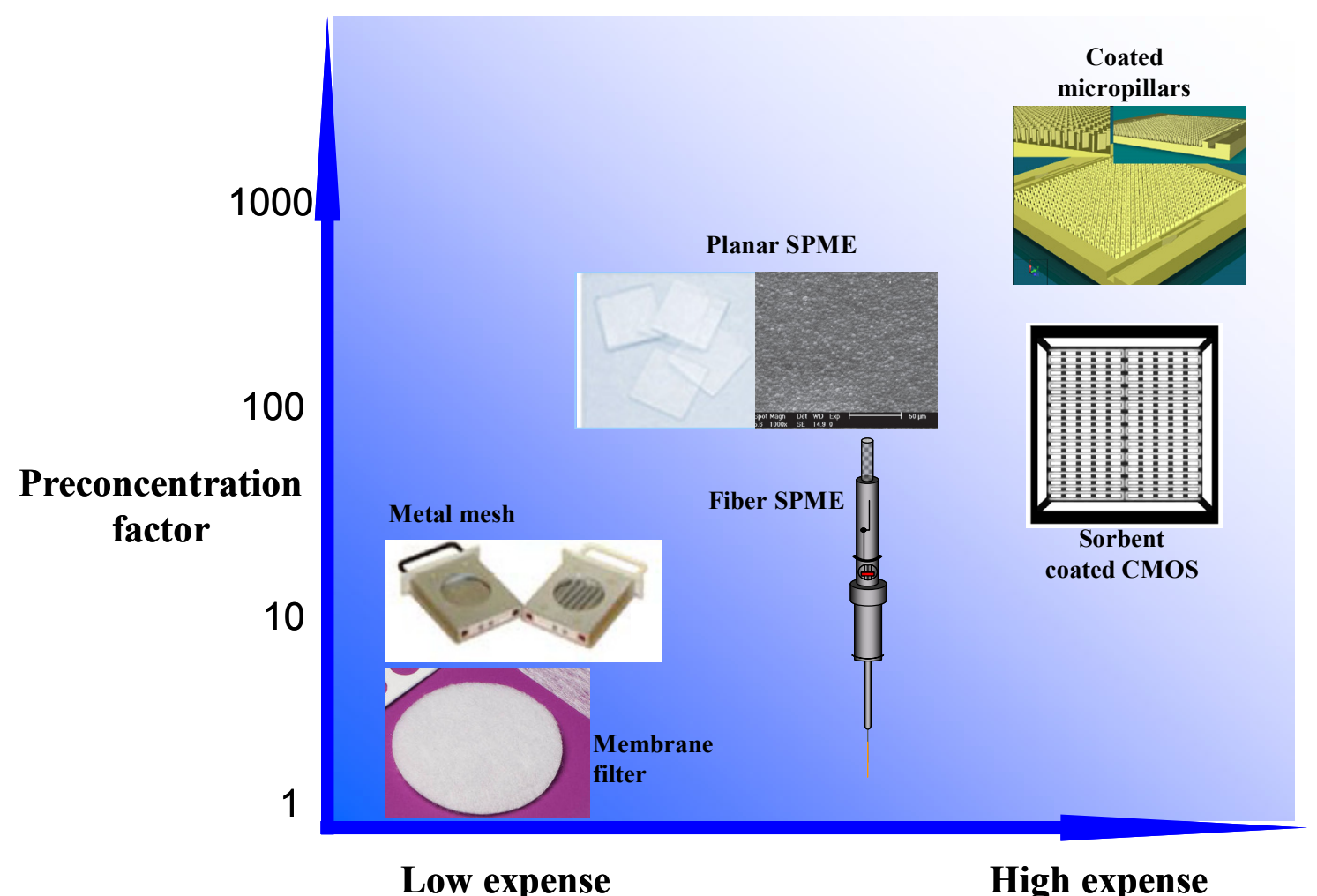

Figure 8. Relative performance of fiber SPME device compared to other devices based on preconcentration factor and cost of manufacture.

\subsubsection{Theory of Solid Phase Microextraction}

Solid phase microextraction is a well developed extraction and preconcentration technique, and is commonly used for both laboratory and field sampling of semi-volatile and volatile compounds. A SPME device is composed of the SPME syringe and the fiber assembly as shown in Figure 9. To sample a closed container, the SPME needle pierces through a septum of the container containing the sample, and the plunger is pushed into gear and locked at the "Z" slot 
to expose the SPME fiber coating. When the fiber coating is exposed to the sample matrix, the extraction process begins.

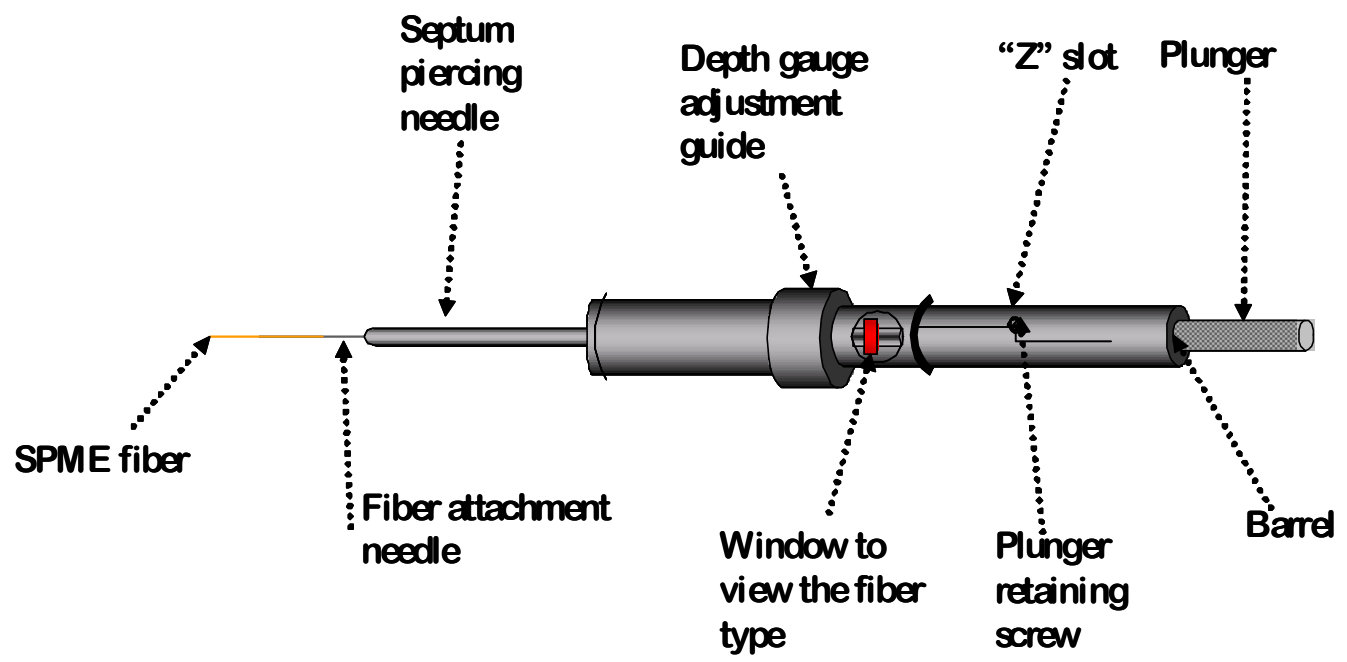

Figure 9. SPME syringe and fiber assembly.

During extraction, volatile or semi-volatile compounds are extracted either by absorption or adsorption onto a non-volatile polymeric coating or onto a solid sorbent phase coating until equilibrium is reached or until the fiber is withdrawn into the needle. Once sampling is completed, the analytes can be thermally desorbed into an inlet of an analytical instrument for analysis. The type and amount of analyte extracted from a sample matrix in a given time depends on the thermodynamic and kinetic properties of the whole system, and on the fiber's chemistry. The remainder of this section aims to give a brief background on the thermodynamics and kinetics of SPME, as well as the different available fiber coating chemistries.

\section{Thermodynamics}

Solid phase microextraction is a multiphase equilibrium process. For simplicity, only three phases will be considered: the SPME fiber coating, the gaseous phase above a sample, and the sample (in solid or liquid form). During extraction, if adequate time is given, sample 
molecules move between the three phases until equilibrium is reached. Mass is neither lost of created in a close system, so Equation 1 holds for the SPME system. Where $C_{0}$ is the initial concentration of the analyte in the sample matrix while $C_{f}^{\infty}, C_{h}^{\infty}$ and $C_{s}^{\infty}$ are the equilibrium concentrations of the analyte in the coating, the headspace gas and the sample matrix, respectively. $V_{f}, V_{h}$, and $V_{s}$ are the volumes of the SPME coating, the headspace gas, and the sample matrix, respectively.

$$
C_{0} V_{s}=C_{f}^{\infty} V_{f}+C_{h}^{\infty} V_{h}+C_{s}^{\infty} V_{s}
$$

Equation 1

The distribution constant between the fiber coating and the headspace gas can be defined as $K_{f h}=C_{f}^{\infty} / C_{h}^{\infty}$, and distribution constant between the headspace gas and the sample matrix as $K_{h s}=C_{h}^{\infty} / C_{s}^{\infty}$, and the mass of the analyte absorbed/adsorbed by the fiber coating as $n=C_{f}^{\infty} / V_{f}$, then the following equation is true for the mass extracted:

$$
n=\frac{K_{f h} K_{h s} V_{f} C_{0} V_{s}}{K_{f h} K_{h s} V_{f}+K_{h s} V_{h}+V_{s}}
$$

By establishing the connection between the distribution constants with the Henry's constants for each of the 3 distribution constants above, $K_{f h}$ can be written as $K_{f h}=K_{f s} / K_{h s}$. By substituting $K_{f h}$ into Equation 2, the mass of analyte on the fiber can be written as:

$$
n=\frac{K_{f s} V_{f} C_{0} V_{s}}{K_{f s} V_{f}+K_{h s} V_{h}+V_{s}}
$$

If the container holding the sample is assumed to be completely full, such that $V_{h}$ is very close to zero, then the second term in the denominator drops and Equation 3 can be simplified to: 


$$
n=\frac{K_{f s} V_{f} C_{0} V_{s}}{K_{f s} V_{f}+V_{s}}
$$

When the container is assumed to be mostly empty headspace, such that $V_{s}$, the volume of the sample is very close to zero, then the nominator Equation 3 becomes infinitely small and causes $n$ to approach zero. Thus, $n$ becomes dependent on the initial $V_{s}$ as $V_{s}$ becomes significantly smaller than $V_{h}$. This scenario is more correctly true for field sampling environments, where $V_{s}$ is infinitely small compared to $V_{h}$.

Parameters that can affect the extraction performance of SPME are: temperature, salting, $\mathrm{pH}$, thermal swelling, and polarity of coating material and of analyte, of which each can alter the $K_{f s}$ in Equation 4 to some degree. Details on these parameters (except for the last one) are not described in this dissertation because the SPME extraction experiments of explosives and drugs are performed under un-modified environment conditions for practical field application.

\section{Kinetics}

The rate of SPME extraction process depends on the mass transport phenomenon. The diffusion coefficient of the analyte between the phases is described by Fick's second law of diffusion. SPME extraction can either be direct extraction or headspace extraction. In the direct extraction setup, the SPME fiber coating is submerged into an aqueous sample, hence a boundary layer exists between the liquid and the fiber coating as shown in Figure 10 (left). The diffusion effect gives rise to the linear concentration profile at the boundary layer. In the headspace extraction setup, the SPME fiber sits in the headspace air of the container, above the sample matrix. The analyte undergoes a series of transportation processes, from the sample matrix to the 
gas phase, then from the gas phase into the SPME fiber coating. These processes continue until the system reaches equilibrium, at which point the concentrations of the analyte in the 3 phases are constant. In headspace extraction systems, the concentration profile of the analyte is different from the direct extraction systems. The concentration profile for the headspace technique is constant with distance, while the coating and the sample matrix phase have non-constant and nonlinear concentration profiles.[64]

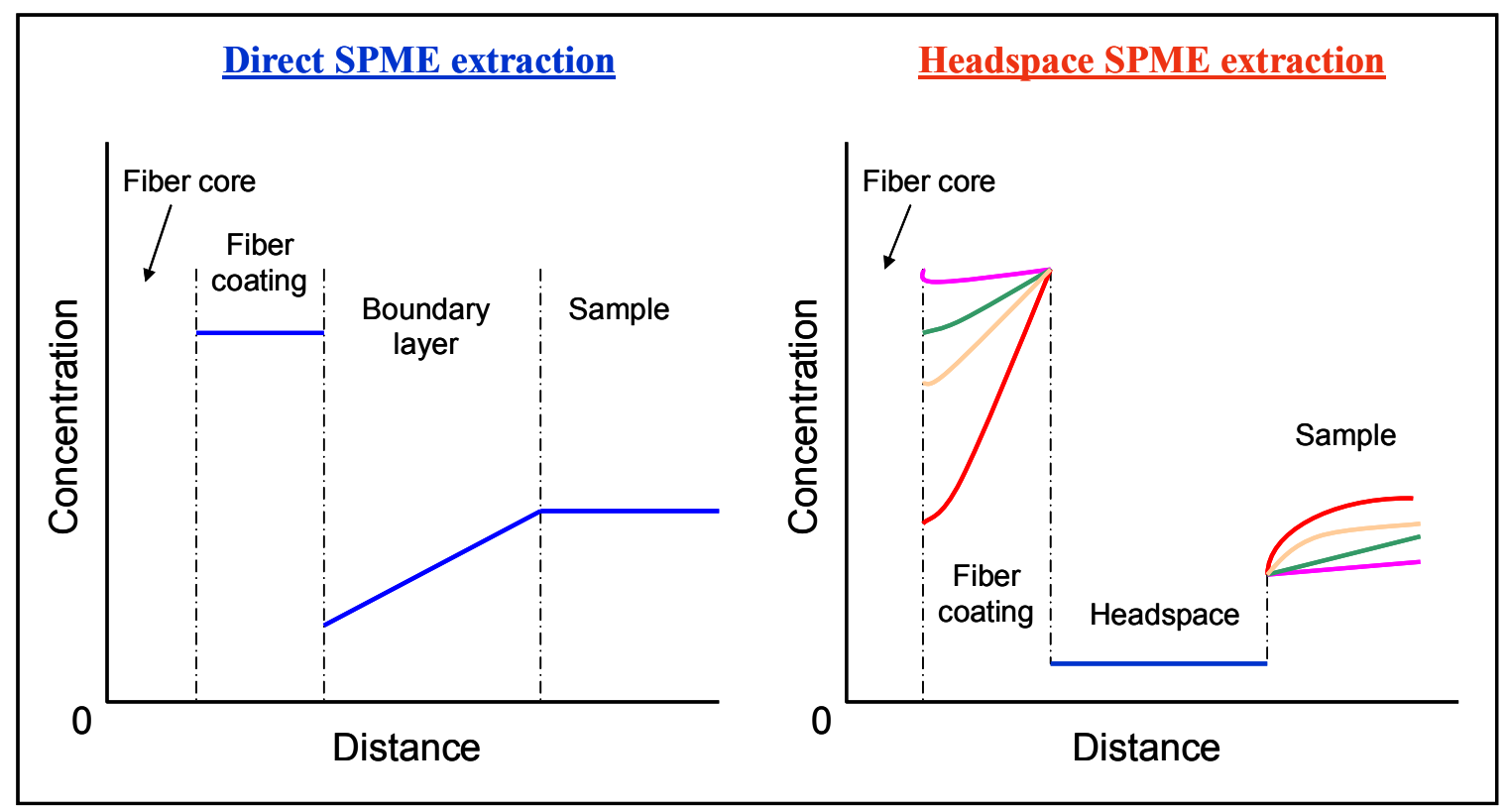

Figure 10. Headspace SPME extraction vs. Direct SPME extraction showing the concentration profiles of the analyte between phases with respect to distance.[64]

Direct extraction and headspace extraction can be performed both in a static environment (without gas/fluid flow) or in a dynamic environment (with gas/fluid flow). This dissertation was performed using static headspace extraction technique for all explosives and drugs samples since the aim of the study is to determine which analytes are extractable under the most simple, nonagitated conditions for IMS detection. Studies on improving extraction efficiency, and comparing 
static vs. dynamic SPME setups, are being conducted by other members of the Almirall research group.

\section{Fiber Coating Chemistry}

Depending on the nature of the analyte being extracted (i.e. polarity, molecule weight, and volatility), a given coating chemistry is preferred over the others. The extraction time and the sensitivity are function of both the coating thickness and the distribution constant of a specific analyte between the gas phase and the coating matrix. Poly(dimethylsiloxane) (PDMS) is the most popular coating to date because it is a very rugged liquid polymer coating, is not easily stripped, and is able to withstand high temperatures. Although PDMS is a non-polar phase (extremely effective for extracting non-polar analytes), it can also adequately extract slightly polar compounds. Poly(acrylate) or (PA) on the other hand is a solid polymer at room temperature, more suitable for polar compounds, but has lower diffusion coefficient compared to PDMS, and hence longer extraction times for volatile analytes. Carbowax $(\mathrm{CW})$ is another polar coating, a solid sorbent rather than liquid, thus it has smaller dynamic range and suffers from displacement effects.

Mixed phase coatings are also very common because they offer complementary properties compared to a single coating. For example, poly(divinylbenzene) (DVB) usually comes as porous particles embedded in a PDMS or Carbowax coating to create mixed phase chemistry. When the sample matrix involves a broad range of compounds, mixed phase coatings usually perform better than single phase coatings. Table 10 lists the fiber chemistries available for the extraction of the volatiles from drugs and explosives samples in this dissertation. Further experimentation with these different SPME fibers is necessary to compare the performance among the different chemistry coatings for the target analytes. 
Table 10. Commercially available SPME fiber coatings.

\begin{tabular}{|l|l|l|}
\hline \multicolumn{3}{|l|}{ Fiber type } \\
PDMS & Absorbent & Non-polar \\
\hline CAR/PDMS & Adsorbent & Bipolar \\
\hline PDMS/DVB & Adsorbent & Bipolar \\
\hline CW/DVB & Adsorbent & Polar \\
\hline DVB/CAR/PDMS & Adsorbent & Bipolar \\
\hline
\end{tabular}

\subsection{Developing Technologies}

The importance of effective sampling for low concentration vapors encourages the development of new preconcentration devices. Some of the developing preconcentration devices reported in recent literature are based on the following technologies: MEMS sorbent-coated micro-heaters with micro-valves, mass sensitive MEMS, micro-pillar inkjet printing deposits, nanoporous-carbon film deposits, and planar SPME. The following paragraphs briefly describe these emerging preconcentration devices.

Derived from the same CMOS technology, but different from the MEMS device from the US Naval Research Laboratory, Yeom et. al. took the sorbent-coated micro-heater MEMS preconcentration technology to the next level by monolithic integration of the micro-valves into the micro-heater of the device. Integration of the micro-valves right next to the inlet and outlet of the preconcentration chamber reduces the dead volume significantly and is expected to improve sensitivity of the method.[65] Thus far, no data have been presented on preconcentration of explosives and drugs using this method. 
Recently, a mass-sensitive MEMS preconcentrator for use in chemical trace detection was developed by Manginell et. al. The device is called a smart preconcentrator (SPC) because it not only preconcentrates analytes but also senses the mass collected as function of time, and notifies the system when sufficient analyte has been collected for detection by a downstream chemical microanalyzer. The SPC is constructed from a Lorentz-force-actuated pivot-plate resonator with an integrated heater, and is coated with an adsorbent. The frequency of operation varies inversely with the mass of collected analyte. The SPC has been shown to have a limit of detection of less than $50 \mathrm{ppb}$ for a nerve-gas stimulant, dimethyl-methyl-phosphonate (DMMP).[66]

Alfeeli et. al. used microfabrication technology to develop a microthermal preconcentrator device on a $7 \mathrm{~mm} \times 7 \mathrm{~mm}$ silicon-glass chip that hold 3500 micropillars coated with a polymer adsorbent film (deposited by inkjet printing technology). The device has been used with a GC, and PDMS and Tenax TA as adsorbent materials, for a hydrocarbon mixture. These configurations achieved concentration factors of 10 and 1000 respectively.[67]

Nanoporous-carbon films have been grown on various surfaces to any desired thickness, with precise morphology and density for preconcentration devices. Sandia National Laboratory used NPC films in a preconcentration device to adsorb dimethyl methylphosphonate (DMMP) for a gas chromatography sensor system. No literature on preconcentration of explosive and drug vapors using this device has been reported.[68]

As previously mentioned, one drawback of SPME fiber preconcentration devices is their limited absorption and adsorption capacity. To further improve the sensitivity of the SPME device, Guerra et. al. developed a planar SPME geometry, which provides a much larger surface 
area and capacity of absorption or adsorption. The planar geometry sol-gel PMDS SPME device, using IMS as detector, and TNT as test compound, was reported to have a 4 times improvement in sensitivity over commercially used cylindrical SPME fiber geometry. Other coating chemistries for planar SPME are under investigation.[28] The additional advantage of the planar SPME preconcentration device is that it can be used directly with current commercial IMS systems, without further modification to the front end of the analyzers or the addition of an interface.

This dissertation utilizes solid phase microextraction because the sampling and preconcentration capabilities of SPME are essential for comprehensive trace detection by improving the limit of detection of a range of volatile compounds that can be used in multi-channels IMS detection algorithms. This results in lowered false alarm rates and increased probability of detecting hidden explosives and illicit drugs. With the availability of the fiber SPME-IMS interface, different commercial SPME fibers can be evaluated to determine which coating meets the objective of extracting the target volatile signatures from the explosives and drugs in this study. 


\section{CHAPTER 7: THEORY AND BACKGROUND OF IMS}

CONTENT

7.1 IMS Detection Theory

7.2 Ion Mobility in Gases at Atmospheric Pressure

7.3 Operational Parameters Affecting IMS performance 76

7.4 IMS Limit of detection, Linear Dynamic Range, and \% RSD 82

7.5 Limitations and Future Developments of IMS

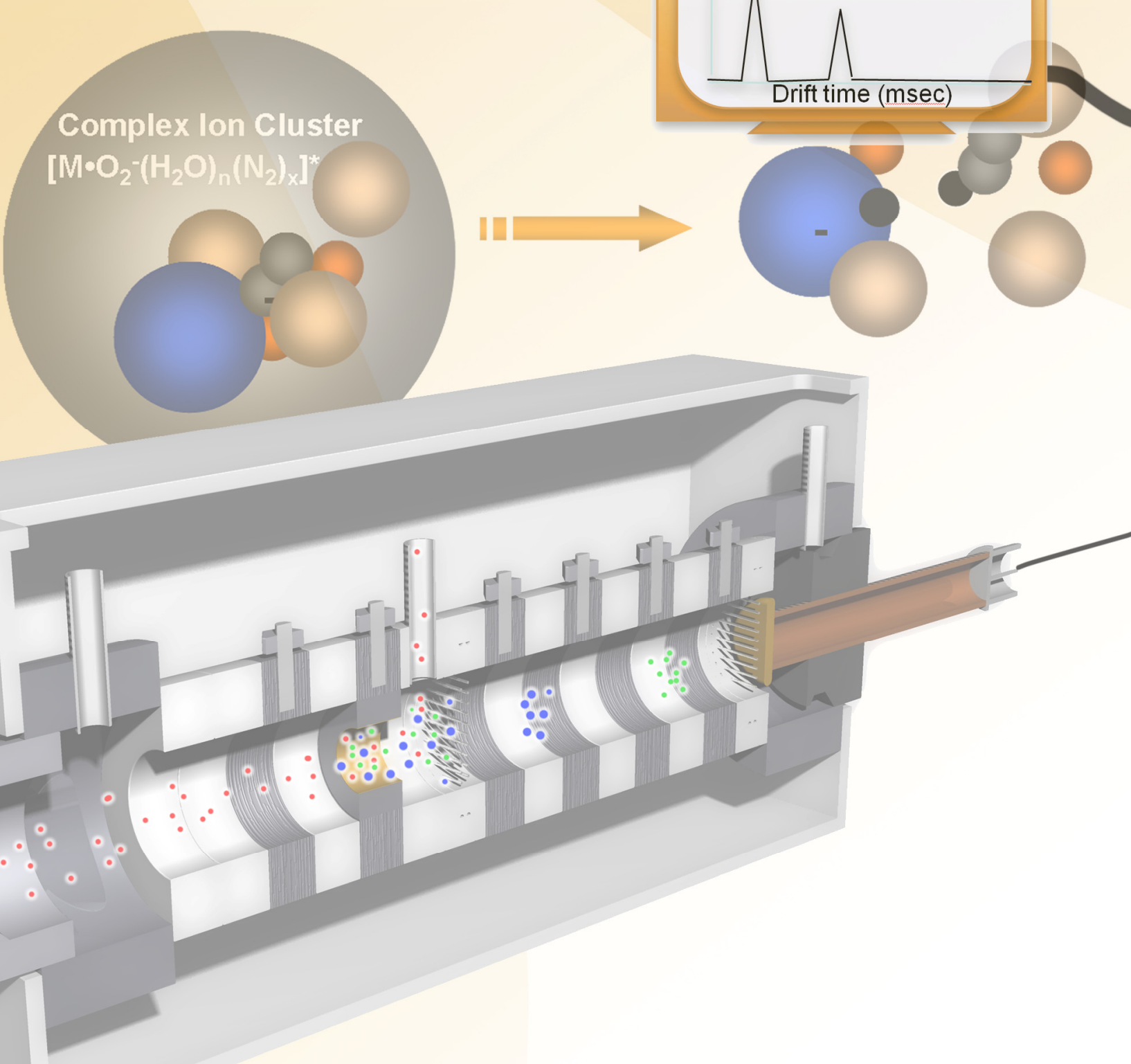


This chapter describes the background theory of ion mobility spectrometry, operational and instrumental design, ion mobility in gases at atmospheric pressure, IMS gas-phase ion chemistry, ion chemistry of explosives and drugs, operational parameters affecting IMS performance, and the future development of IMS. The chapter's structure is illustrated in the flowchart below.

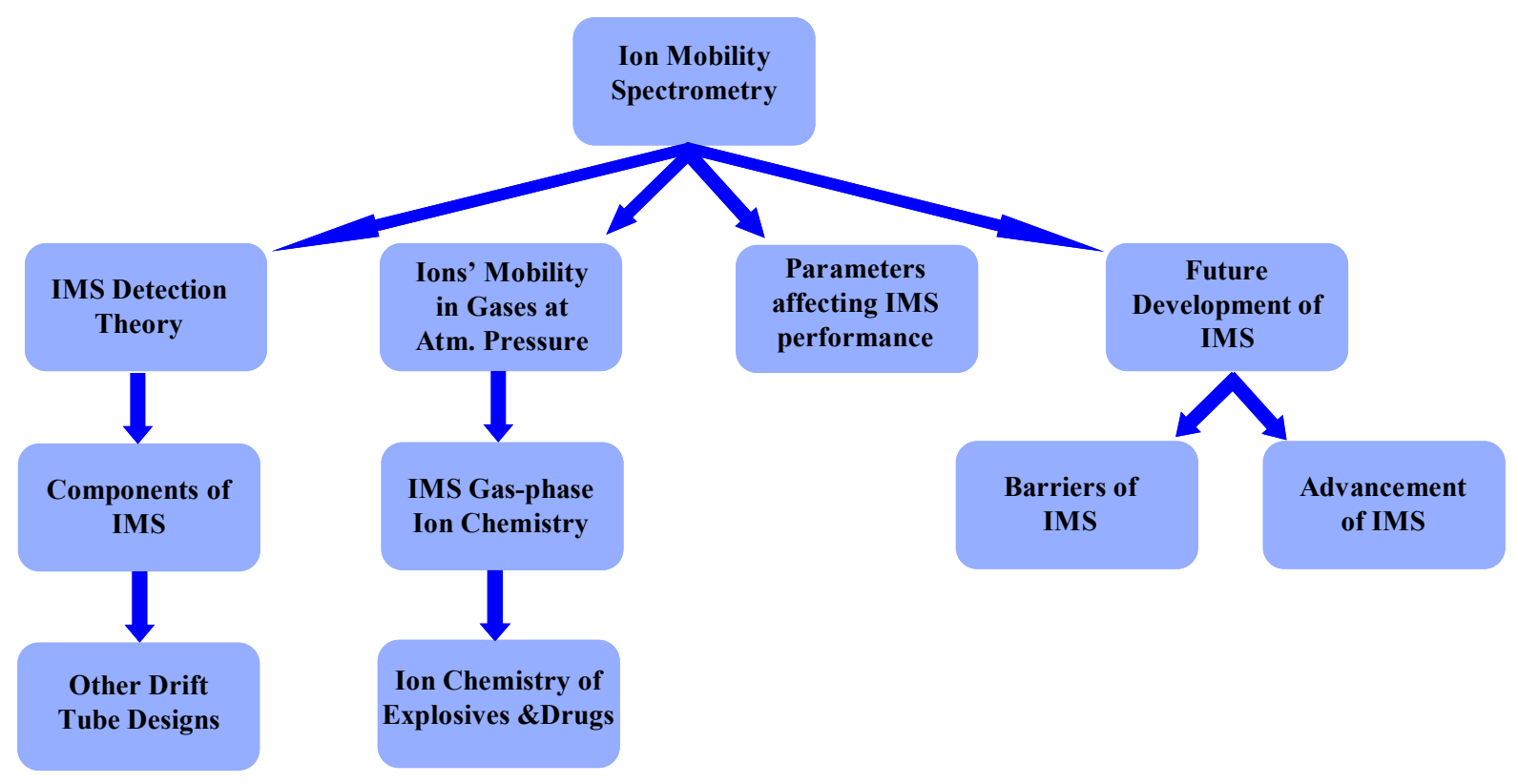

\subsection{IMS Detection Theory}

IMS is an analytical technique that is used for presumptive detection of a substance based on the mobility (drift time or reduced mobility) of the product ions inside an electric field. The analysis process occurs when the sample is brought to the front end of the IMS analyzer, vaporized, and then transported by a carrier gas to the reaction chamber where the gaseous molecules get ionized. The ions that are created are then pulsed into the drift, or separation, region through an ion shutter which acts like a gate. In the drift region the ion swarm travels 
toward the detector under the influence of a weak electric field of approximately $200 \mathrm{~V} \cdot \mathrm{cm}^{-1}$. As the ions drift toward the detector under the influence of this electric field, they are slowed down by collisions with the drift gas molecules traveling in the opposite direction. Ion species with lower reduced mass, smaller collision area, and higher charge would reach the detector faster. The ions' relative arrival times provide a characteristic spectrum used for presumptive identification of the substances present. Ions are detected as they collide with, and are neutralized by, a Faraday plate detector. The collisions produce a very small change in current, which is amplified by an external current amplifier. This signal is synchronized to the gate pulse yielding a mobility spectrum, which is a plot of ion current versus ion drift time as shown in Figure 11.[17]

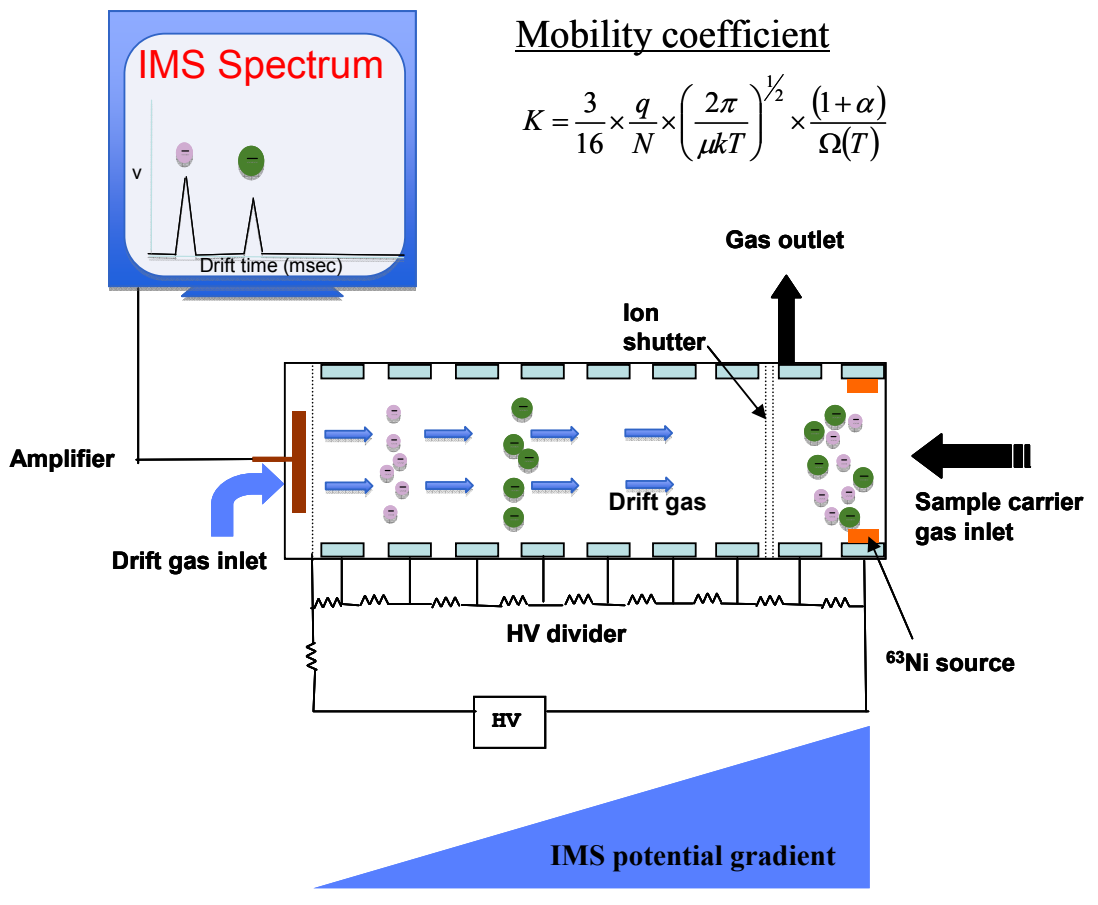

Figure 11. Schematic of a low field conventional stacked IMS and the ion mobility coefficient.

The characteristic average drift velocity $\mathrm{v}_{\mathrm{d}}\left(\mathrm{cm} \cdot \mathrm{s}^{-1}\right)$ of the ion species is governed by the electric field $\mathrm{E}\left(\mathrm{V} \cdot \mathrm{cm}^{-1}\right)$, and mobility coefficient (constant), $\mathrm{K}\left(\mathrm{cm}^{2} \cdot \mathrm{V}^{-1} \cdot \mathrm{s}^{-1}\right)$ through $\mathrm{v}_{\mathrm{d}}=\mathrm{K} \cdot \mathrm{E}$. 
When all the instrument parameters and chemistries inside the drift tube are controlled, the mobility coefficient $\mathrm{K}\left(\mathrm{cm}^{2} \cdot \mathrm{V}^{-1} \cdot \mathrm{s}^{-1}\right)$ is governed by the size-to-charge ratio and the reduced mass of the ion in the supporting atmosphere. Thus the distinction between different ion species is based on their mobility coefficient. The mobility coefficient can be expressed as the reduced mobility, $\mathrm{K}_{0}\left(\mathrm{~cm}^{2} \cdot \mathrm{V}^{-1} \cdot \mathrm{s}^{-1}\right)$ in Equation 5 observed at standard pressure and temperature.[17]

$$
K_{o}=K\left[\frac{273}{T}\right]\left[\frac{P}{760}\right]
$$

\subsubsection{IMS Components}

As illustrated in Figure 11 above, an IMS instrument is comprised of six major subcomponents: a sample inlet, an ionization source, an ion shutter, a drift region, an aperture grid, and a detector. Each of these components and the various applicable technologies are described in detail in Sections 7.1.2.1 to 7.1.2.6, respectively.

\subsubsection{Inlets}

There are several sample inlet designs available for use to deliver samples into the IMS drift tube.[17, 69] These include membrane-based inlets,[70] semi-permeable membrane inlets, spray and electro-spray, laser ablation vaporization, and thermal vaporization which is the most common technique in commercial IMS instruments.[18,71] Research instruments can be equipped with any of these inlets depending on the sample matrix and analytes of interest. An inlet design must satisfy three general requirements. Firstly it must be able to deliver the sample so that gas-phase ion can be produced. Secondly, it must interface well with the analyzer so that minimal sample loss occurs. Thirdly, it must not distort the chemical information of the sample analytes. 
Membrane-based inlets and semi-permeable membrane inlets are thin synthetic polymer films through which organic analytes can diffuse while moisture and dust particles are kept out. IMS instruments operated in complex field environments are usually equipped with a membrane inlet to keep the internal atmosphere of the analyzer isolated from the ambient air being sampled. However, the tradeoff for increased ruggedness is lower sensitivity because analyte detection is governed by the solubility of the analyte in the membrane. Hence the performance of a membrane-based inlet IMS can be affected by external factors such as temperature, vapor pressure of the analyte, and polarity of the membrane and the analyte.[17, 70, 72]

Spray and electrospray inlets can be used for introducing liquid samples into the IMS analyzer. The liquid must be volatilized into gas-phase molecules and subsequently ionized. Although this liquid spray is a simple concept to implement, the gas volume produced from a 1 $\mathrm{mL}$ liquid sample can quickly overload the analyzer. Therefore liquid spray inlets are not a very promising inlet solution. Electrospray inlets, on the contrary, can introduce liquid samples with a small liquid flow $\sim 50 \mathrm{uL} \cdot \mathrm{min}^{-1}$, nebulized through a syringe needle or a capillary opening. This nebulizer is placed at high voltage within a stream of inert gas, forming ion from the analyte molecule.[17] Thus, electrospray can serve both as a liquid sample inlet and also an ionization system. Electrospray is especially useful for nonvolatile and thermo liable compounds that are not suited for thermal vaporization, and has been described for IMS applications.[73-76]

Laser desorption introduces a solid sample into the IMS analyzer by using a laser to heat and vaporize the solid sample into gaseous molecules. Lasers at different energy levels can desorb as well as ionize analytes. IMS instruments equipped with a laser desorption and ionization system can be operated without an ion shutter since the laser can be pulsed to provide a reference for measuring the drift time of the ion swarms. Laser desorption is useful for ablating 
large, heat sensitive biological molecules where other sample inlet methods are not suitable. However, laser methods cause band broadening because of the prolonged reactions in the gas phase over the plume as well as the effect of Coulomb repulsion in a confined volume.[17]

Thermal vaporization is the most common method for explosive and drug detection applications. The desorber can rapidly heat the sample collected (solid particles) or spiked (aqueous solution) on a substrate, to high temperatures between $150{ }^{\circ} \mathrm{C}$ and $300{ }^{\circ} \mathrm{C}$ to form gaseous molecules, that are directed to the reaction chamber. Thermal vaporization inlets are well adapted to commercial IMS instruments because heated desorbers are simple to design, inexpensive to produce, and portable.[69] However, large and thermal liable compounds that are not easily vaporized without a risk of decomposing should be introduced using other techniques (e.g., electrospray).

\subsubsection{Ionization Sources}

Ionization of gaseous molecules in the reaction chamber on the IMS can be accomplished in a variety of ways, including radioactive bombardment, $[17]$ corona discharge, $[77,78]$ photoionization (UV, lamps, laser beam),[79, 80] electrospray ionization,[74-76] surface ionization,[81] and microplasma ionization. $[82,83]$ All of these methods are as effective as radiation bombardment, depending on the applications. The effectiveness of the ionization scheme determines the sensitivity and linear dynamic range (LDR) of the detection system. Table 11 lists the ionization sources that have been demonstrated for use with IMS instruments thus far. 
Table 11. Performance of various ionization sources for IMS detection systems.

\begin{tabular}{|c|c|c|c|c|}
\hline $\begin{array}{l}\text { Ionization } \\
\text { source }\end{array}$ & $\begin{array}{c}\text { Ions } \\
\text { (RIP, +/-) }\end{array}$ & LOD / LDR & Test compounds & Ref. \\
\hline${ }^{63} \mathrm{Ni}$ & RIP, +/- & $20 \mathrm{ppb} / 2$ orders & Explosives and drugs & [17] \\
\hline $\begin{array}{l}\text { Corona } \\
\text { discharge }\end{array}$ & $+/-$ & $2 \mathrm{ppb} / 4$ orders & TNT, PETN, RDX & {$[77,78]$} \\
\hline $\begin{array}{l}\text { Photoionization: } \\
\text { UV, Lamps } \\
\text { Laser }(\mu \mathrm{J}) \\
\text { SELDI }(\mu \mathrm{J})\end{array}$ & $\begin{array}{l}+ \\
\mathrm{MH}^{+} \text {or } \mathrm{MH}^{+} \\
\mathrm{MH}^{+}\end{array}$ & $\begin{array}{l}3 \mathrm{ppb} / 3 \text { orders } \\
100 \mathrm{ppb} / 1-3 \text { orders } \\
2 \mathrm{ppm} / \mathrm{NA}\end{array}$ & $\begin{array}{l}\text { Ketones, alcohols } \\
\text { Naphth., toluene, benzene } \\
\text { Verapimal }\end{array}$ & $\begin{array}{l}{[79,80]} \\
{[84,85]} \\
{[86]}\end{array}$ \\
\hline Electrospray & $\begin{array}{l}- \\
+\end{array}$ & $\begin{array}{l}15-200 \mathrm{ppb} / 2 \text { orders } \\
15-70 \mathrm{ppb} / 2-3 \text { orders }\end{array}$ & $\begin{array}{l}\text { Explosives } \\
\text { Amphetamines, Opiates }\end{array}$ & $\begin{array}{l}{[74]} \\
{[75,76]}\end{array}$ \\
\hline $\begin{array}{l}\text { Surface } \\
\text { ionization }\end{array}$ & + & $1 \mathrm{ppb} / 4-5$ orders & $\begin{array}{l}\text { Narcotics: Nitrogen- } \\
\text { containing organic bases }\end{array}$ & [81] \\
\hline Microplasma & RIP, +/- & $5 \mathrm{ppb} / 2$ orders & $\begin{array}{l}\text { 2-Et-1-Hexanol, 2- } \\
\text { Nonanone }\end{array}$ & [83] \\
\hline
\end{tabular}

$\mathrm{A}^{63} \mathrm{Ni}$ radiation source is the most common ionization source in commercial IMS instruments because it provides stable and reliable operation as well as consistency in ion chemistry. It is the best understood ionization source, hence it has a large library of detectable compounds and the corresponding ion chemistry. $\mathrm{A}^{63} \mathrm{Ni}$ source is a stand-alone component and does not require an external supply, moving parts or maintenance. However, there is an equivalent upkeep cost associated with periodic leak tests. The source is constructed by having ${ }^{63} \mathrm{Ni}$ radioactive material electroplated onto a nickel or gold metal foil, with activity between 10 to $15 \mathrm{mCi}$. The electrons emitted from the ${ }^{63} \mathrm{Ni}$ source carry an average energy of $17 \mathrm{keV}$. The electrons continuously ionize gaseous molecules in the supporting air to form reactant ions, which are then available to react with the sample gaseous molecules to form product ions. However, the rate of the reactant ion formation is much slower than the rate of consumption, which is evidenced by the low linear dynamic range of the ${ }^{63} \mathrm{Ni}$ source IMS ( 0 to $100 \mathrm{ng}$ ). It is common to dope the ${ }^{63} \mathrm{Ni}$ reaction region with a reactant gas other than air, which is of higher proton affinity or electronegativity to improve the selectivity of product ion formation. Further details on dopant 
gas are described in Section 7.2.1. Radioactive materials other than ${ }^{63} \mathrm{Ni}$ have been used, including tritium and ${ }^{241} \mathrm{Am}$, although the use of radioactive sources is discouraged today due to legal complications such as permits and licensing procedures.[17]

Corona discharge ionization sources can be constructed using a continuous or a pulsed source of DC power to create a potential difference between a sharp needle and an opposing electrode. The needle and the electrode are placed $\sim 2 \mathrm{~mm}$ apart at voltage difference of $\sim 2000 \mathrm{~V}$ to develop an electrical discharge in the gap, creating large number of electrons. $[17,77,78]$ In the supporting air atmosphere, both negative and positive ions can be formed. It has been reported that the total ion current produced is about 10 to 100 times greater than that of the ${ }^{63} \mathrm{Ni}$ source and that a lower detection limit and wider linear dynamic range (0 to 10,000 ng) are achieved.[77, 78] Corona discharge ionization sources have been considered valuable because there is no radioactivity, they are simple to construct, and they are especially useful for direct analysis of liquid samples. However, corona discharge sources are not favored in field IMS instruments because of several disadvantages including the need for an extra high voltage power supply, maintenance of the discharge surfaces, and off-gassing from corrosion affecting the ion chemistry.

Photoionization uses photo-discharge lamps that emit photons (at energies between 9 to $12 \mathrm{eV}$ ) to ionize neutral molecules at atmospheric pressure. Positive molecular ion is formed by losing an electron to become $\mathrm{M}^{+}$, while negative molecular ion is formed as a result of the free electron going through series of associated reactions. UV-photoionization is commonly used for analyzing aromatic hydrocarbons,[17] alcohols,[79] and ketones[80] in laboratory IMS instruments. Photoionization can also be achieved with lasers, where samples are vaporized during the laser desorption process, immediately followed by ionization. Matrix-assisted laser 
desorption ionization (MALDI) and surface enhanced laser desorption ionization (SELSI)[86] also fall under this category. Thus far, little work has been reported on the analysis of explosives and drugs using IMS with photoionization methods. This is mostly because of the cost of replacing the lamps is high, and the public safety concerns make field operations challenging.

Electrospray ionization has been previously mentioned in the sample introduction technique above. The formation of ion occurs when a liquid sample is sprayed from a needle tip under high voltage to form an aerosol. Electrospray ionization IMS is a great technique for laboratory analysis of environmental and biological liquid samples and has also been widely used for analysis of explosives and drugs.[74-76] However, ESI IMS suffers from low sample throughput because of the rinsing time required to flush out the previous fluid matrix to avoid carryover, limiting its suitability for IMS applications in high throughput field detection.[17]

Ionization in IMS can also be performed through surface ionization, where the source is made from a single crystal of molybdenum mixed with iridium. Analyte molecules can undergo electron transfer upon collision with the surface. The ionization efficiency of the source is analyte dependent, and while sources from other substances can be made, surface ionization sources are very effective at ionizing nitrogen-containing organic bases. However, they are not as universal compared to other ionization techniques.[17, 81]

Finally, dielectric barrier discharge (DBD), a method of forming microplasma has been recently used as ionization source for IMS instruments. The discharge probe is composed of a glass capillary, that provides a He gas flow, and silver wires wrapped around the capillary forming electrodes separated at a $12 \mathrm{~mm}$ distance. The discharge is initiated between the electrodes when a periodic positive voltage pulse $(5.5 \mathrm{kV}$ with a frequency of $33 \mathrm{kHz}$ and a pulse 
width of $2 \mu \mathrm{sec}$ ) is applied to one electrode while the other electrode is kept isolated. This discharge forms a plasma outside the capillary, and ions from the plasma initiate an ion-reaction chain leading to the formation of the same reactant ions that are available from the traditional ${ }^{63} \mathrm{Ni}$ ionization source; $\mathrm{H}^{+}\left(\mathrm{H}_{2} \mathrm{O}\right)_{n}$ and $\mathrm{O}_{2}^{-}\left(\mathrm{H}_{2} \mathrm{O}\right)_{n}$. The microplasma ionization source has a comparable linear dynamic range to the ${ }^{63} \mathrm{Ni}$ ( 2 order of magnitude), and $\sim 4$ times better detection limits.[82, 83]

\subsubsection{Ion shutters}

When a continuous ionization source is used in the IMS, an ion shutter (gate) must be in place to allow ion swarms to pass through at a fix interval. The triggering of the gate opening is synchronized with the start of the data acquisition process and is the basis for measuring drift times of the different ion species from the ion shutter to the detector. All commercial stacked drift tube IMS instruments that contain the ${ }^{63} \mathrm{Ni}$ continuous ionization source are equipped with the conventional Bradbury-Nielsen gate (BNG). The BNG is consists of two electrically isolated parallel sets of wires (as shown in Figure 12) held at the same potential $\mathrm{V}_{\text {ref }}$ when the gate is open, and switched to different potentials when the gate is closed. When the gate is closed a voltage offset is applied to each set of wires such that the new voltage for $\mathrm{W}_{1}$ is $\mathrm{V}_{\text {ref }}+\mathrm{V}_{\text {offset }}$, and the new voltage for $\mathrm{W}_{2}$ is $\mathrm{V}_{\text {ref }}-\mathrm{V}_{\text {offset }}$. The $\mathrm{V}_{\text {ref }}$ is kept fixed relative to the location of the gate

along the drift tube $\mathrm{HV}$ gradient, while the $\mathrm{V}_{\text {offset }}$ is applied from a separate power supply. $\mathrm{V}_{\text {offset }}$ is chosen to create a potential field between each alternating wire that is 2 to 3 times stronger than the drift tube field.[17] Alternatively, the performance of a BNG set at a given $\mathrm{V}_{\text {offset }}$ can be tested and observed in an ion optic simulation SIMION (described in CHAPTER 8:). 


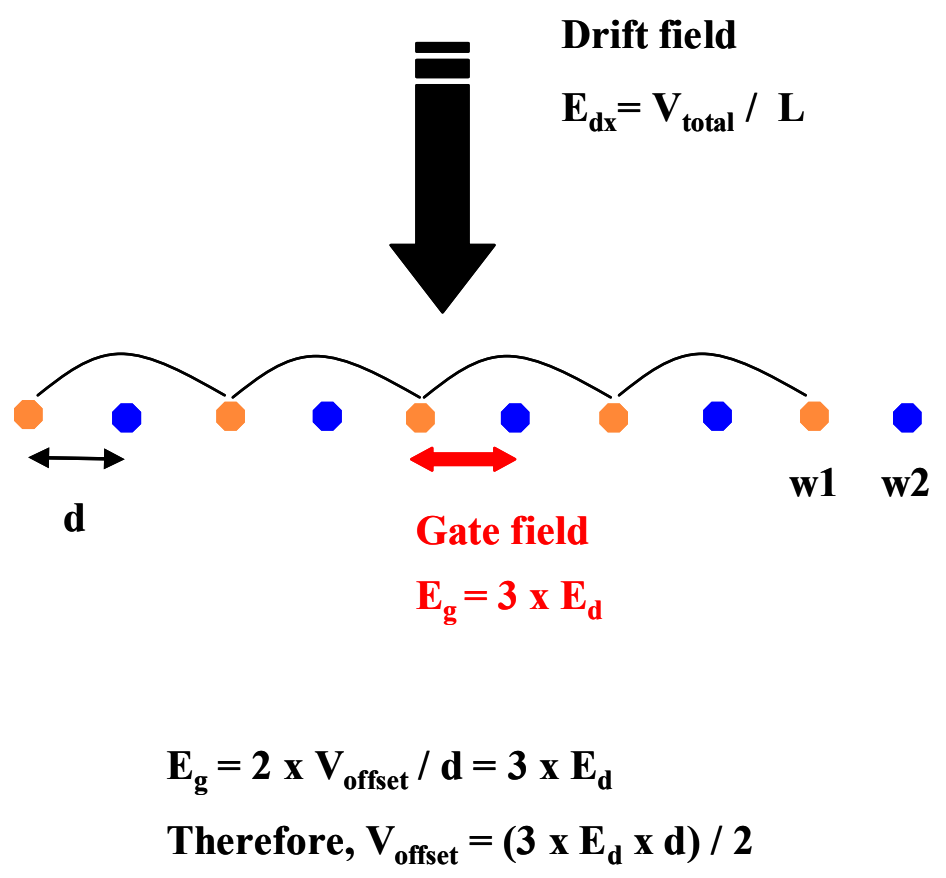

Figure 12. Electrical fields at the Bradbury-Nielsen gate created by the offset in voltage between the two sets of wire.

When the offset voltage is on, the gate field imposes a deflection angle on the ion's path, such that the ion is either directed toward the gate wires and neutralized or collide with the drift tube's wall before they reach the detector. Thus no ions get through when gate is closed. The deflection angle can be calculated from the equation shown in Figure 13, with $V_{0}$ being the $V_{\text {ref }}$, and $\mathrm{V}_{\mathrm{p}}$ is the $\mathrm{V}_{\text {offset }}$ or $\mathrm{V}_{\text {bias. }}$. If the voltage on the wires changes when ions are in the deflection region, the ions experience partial deflection, which leads to blurring of the deflection angles at the rising and falling edges of the voltage pulses. Better timing responses can be achieved by reducing the wire spacing (d) and thus the length of the deflection region. Reducing the wire spacing also decreases offset voltage necessary to achieve a sufficient gate field and deflection angle.[87] 


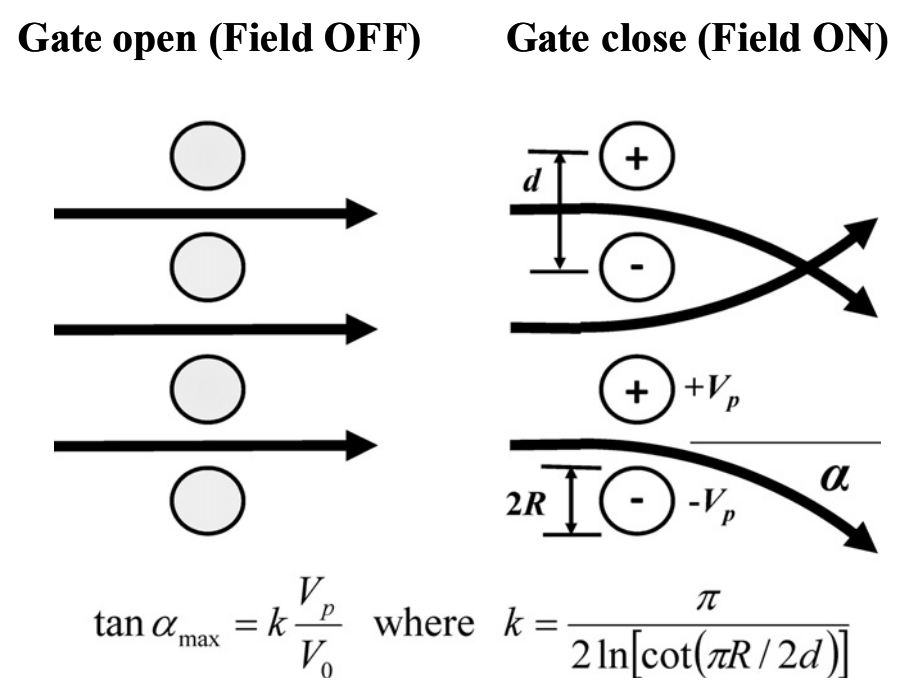

Figure 13. Deflection angle of ions in proximity of the Bradbury-Nielsen gate.[87]

The main disadvantage of reducing BNG wire spacing is the increased complexity of fabrication as well as decreasing passage for the ions. The main challenges in reducing the spacing are to precisely position the alternating set of wires, keep them electrically isolated, and maintaining tension in the wires to keep them wrinkle free.

There are many different ways to fabricate a BNG, with innovations focusing on improvements in the wiring method that reduce labor time from days to hours. Some of the earlier methods involved weaving against the threads on nylon bolts, etching a thin metal foil, and wirebonding to metal deposited on ceramic frame. The most common method is to weave wires into two sets of holes on an insulating frame with a wire spacing of $1 \mathrm{~mm}$.[88] Kimmel et al. developed a V-grooved gate fixture that is machined to have $0.1 \mathrm{~mm}$ wire spacing. The wires are threaded under a microscope in 3 hours time.[89] Recently, Zuleta et al. developed a microfabricated BNG with wire spacing as small as $15 \mu \mathrm{m}$. This microfabircation method takes a day since it uses deep reactive ion etching of silicon-on insulator substrates.[90] The microfabricated design is an important improvement because it reduces wire spacing, which is 
very valuable for miniaturized IMS where ion beams are only one millimeter in size and would otherwise pass through a $\mathrm{BN}$ gate with $1 \mathrm{~mm}$ wire spacing.

Bradbury-Nielsen gates are usually opened to allow ion swarms to pass through for 200 $\mu \mathrm{sec}$ in a $20 \mathrm{msec}$, a duty cycle of only $1 \%$. Short opening times limit the sensitivity of an IMS, but if the gate width increases, peak broadening would result in poor resolution. The limitation of small duty cycle encouraged the development of other gate designs differing from the BradburyNielsen gate. There are ion shutter designs such as the square waveform developed to improve the duty cycle of the gate, which led to the development of Fourier Transform-Ion Mobility Spectrometry. An IMS instrument operating in the Fourier Transform mode requires the following four features. (1) A gating signal generator which produces a binary (on, off) square wave. (2) An entrance and exit gate which are driven by the same gating signal so that the opening and closing time of the gates occurs simultaneously. Both gates are turned on $50 \%$ of the time and off $50 \%$ of the time, so the final duty cycle is $25 \%$. (3) A scanning cycle parameterized to match the square wave frequency. (4) A computer that can record the FT-IMS interferogram and perform a Fourier transformation of the data to the normal time domain which is then plotted as the drift time in ion mobility spectrum. One of the earliest FT-IMS instruments built was from Hill's group which improved the duty cycle to $25 \%$ compared to $1 \%$ by the BN-gate IMS.[91] Nearly a decade later, Sandia National Laboratory showed that it is possible to perform the function of the second gate outside of the drift tube in the electronics, with a simulated, external second gate. The advantages of the external second gate is that it retains the $50 \%$ duty cycle and results in a 7 fold increase in sensitivity.[92] To date, however, this design has not been adopted and commercialized, mostly because FT-IMS would require completely new signal acquisition software and electronics. Other than $\mathrm{BN}$ and square wave designs, no alternative ion shutter designs have been described thoroughly in literature.[17] 


\subsubsection{Drift region}

The drift region of the drift tube is the section between the ion shutter and the detector plate. In the conventional stacked IMS drift tube, this region is composed of a series of alternating conductive and insulating rings (thickness varies with different designs), stacked together to form a tube of any desirable length. It is within this region that different ion species are subjected to a weak electrical field, causing them to move toward the detector. Within the drift region, a drift gas is introduced and made to flow opposite to the direction of ion travel. Ion species thus continuously collide with the counter flow drift gas which causes them to slow down relative to each other depending on their collision cross sectional area. These two phenomena are the basis of ion separation inside the IMS drift tube. The longer the drift region, the more time is given for ion of different species to separate. This increases resolution, but causes a trade off in lower signal intensity. The performance features of an IMS such as resolution, signal to noise ratio, and durability are greatly affected by the materials used for the construction of the tube, and the linearity of the electric field applied to drive the ion swarms toward the detector.[17]

The drift tube conductive rings can be made from metal such as stainless steel, aluminum, brass or even copper. The difference in conductivity between these metals is not a significant design constraint, nor is their cost. Thus the material choice is usually made based on chemical inertness, durability, and ease of machining. Stainless steel is durable but also the hardest metal to machine. It would be most efficient to purchase stainless steel as pre-made rings rather than machine them from rod form. Aluminum is lighter in weight and softer to machine, however its high thermal expansion coefficient makes it unsuitable for IMS instruments that operate at elevated temperatures. Brass and copper are both soft metals, ideal for machining. However, 
brass may tarnish over time, and copper is extremely reactive and adsorptive to organic compounds.[17]

Similar to the conductive drift rings, the choice of an insulator material to make the insulation rings is important. The material chosen must be chemically inert, low cost, and easy to machine. In addition, the insulator must be capable of resisting up to $10^{16} \mathrm{Ohm} \cdot \mathrm{cm}^{-1}$. Some possible insulation materials which can be used for the insulator rings are glass, ceramic, mica and Teflon. Plastic and other materials that tend to off-gas are generally avoided. From the Teflon family of polymers, one of the best candidates is polytetrafluoroethylene (PTFE), which has excellent electrical properties and a high operating temperature of $260{ }^{\circ} \mathrm{C}$. However, PTFE has high thermal expansion coefficient, thus it is recommended the percentage of expansion after heating is accounted for before the rings are machined.

\subsubsection{Aperture grid}

The aperture grid is a simple and necessary component in IMS in order to prevent distortion in the mobility spectrum. The aperture grid creates an electrical shield so that the detector is blind from the induced current flow of the approaching ion swarms in the drift region until they pass though the aperture grid.[17] The grid can be constructed in the form of thin wire mesh or parallel wire grid that is placed approximately 0.5 to $2 \mathrm{~mm}$ away from the detector plate. The aperture grid is biased at a voltage such that the field between the aperture grid and the detector, $E_{a-d}$ is $\sim 2$ to 3 times higher than the drift tube field, $E_{d}$. This field is necessary because if the electrical field strength between the aperture grid and the detector is less than the drift tube field strength, distant ion swarms would induce a current flow that results in peak broadening and distortion. Both theoretical calculations and reported experimental data show that the ion peak 
full width at half maximum (FWHM) and the drift time decreases with increasing field strength between the aperture and the detector.[93] The detected ion current (signal intensity) has also been shown to increase as $\mathrm{E}_{\mathrm{a}-\mathrm{d}}$ increases.[94] The field between the aperture grid and the detector can be adjusted by shortening/lengthening the distance between the aperture grid and detector, or decreasing/increasing the bias voltage on the aperture grid.[17]

\subsubsection{Faraday Detector and Signal Acquisition}

The simplest and most commonly used ion collector for ion mobility spectrometer is the Faraday plate. It is a circular metal disc that is placed at the end of the drift tube directly facing the incoming ions. The disc is connected to the inverting input of a current amplifier. When ions collide and annihilate on the Faraday plate a current, between $\sim 10^{-10}$ to $10^{-11}$ amperes, is produced and amplified to a DC voltage of $\sim 1$ to $10 \mathrm{~V}$ by the current amplifier. The output DC voltage is the measure of ion signal intensity, and is considered to have arbitrary units because it varies between IMS instruments depending on the gain set on the current amplifier.[17]

Output from the detector of an IMS is an analog signal, which is digitized, stored as a text file, and plotted on an x-y graph as the characteristic ion mobility spectrum. The drift time of the ion species is plotted on the x-axis, starting at " 0 " (set corresponding to the time at which the ion shutter opens to allow ion swarms through), and the signal intensity in arbitrary units or DC voltage is plotted on the y-axis. Data from a single spectrum (scan) is usually too noisy for useful analysis, even on fine-shielded instruments. Hence, the ion mobility spectrum is actually obtained by averaging a number of individual scans. It was reported that by averaging " $n$ " similar scans, the signal to noise ratio would improve by a factor of square root of " $n$ ".[17] It is common to collect 100 to 400 individual scans for signal averaging, while others have shown up to 1000 scans. Increasing the number of scans is traded off against the total analysis time required. 


\subsubsection{Alternative Drift Tube Designs and Miniaturization}

Several groups have brought forth the advancements in IMS drift tube design over the past decade, with new configurations that could simplify the construction of drift tubes making mass production at low cost possible as well as improving sensitivity and resolution.

For the IMS using linear low-field drift tube designs, glass and resistive drift tubes have been shown as an alternative to conventional stacked rings drift tubes. Recent attempts at miniaturizing IMS for use in field applications put more constraints on drift tube design, because minimizing the drift tube generally results in reduced performance in terms of sensitivity and resolution. Sandia National Laboratories developed the $\mu$Hound $^{\mathrm{TM}}$ IMS with a miniaturized drift tube $10 \mathrm{~mm}$ OD and $54 \mathrm{~mm}$ in length, constructed from stack of MACOR ${ }^{\mathrm{TM}}$ ceramic dielectric rings with $0.50 \mathrm{~mm} \mathrm{Ni}$ electrodes separated by $0.25 \mathrm{~mm}$ sapphire washers.[95] However, the ion shutters, aperture grids and discrete drift rings used with linear low-field drift tube designs remain as barriers in both IMS miniaturization and mass production because of the high production and maintenance cost these components demand.

Besides the linear low-field IMS technology, there is another closely related technology namely field asymmetric waveform-ion mobility spectrometry (FAIMS), also known as differential mobility spectrometry (DMS). The DMS drift tube technology is based on fielddependent mobility. Ion swarms are transported to the detector via a carrier gas in the analyzer region, which is composed of two parallel plates or a pair of concentric cylinders. One electrode is held at ground, while an RF electrical field $\mathrm{E}(\mathrm{t})$ is applied to the other. Since this field is perpendicular to the direction of the carrier gas flow, ion is shifted toward one electrode at a net distance after the high-field and low-field portion of the waveform at the velocity of $v_{\perp}=\mathrm{K}(\mathrm{E})$ $\cdot E(t)$, where the ion's perpendicular mobility is the function of the electrical field. If its total 
longitudinal displacement after $\mathrm{n}$ periods of the waveform is less than the distance to one of the electrodes, the ion would eventually collide with one of the electrodes and neutralize before reaching the detector. Ion swarms of a given net perpendicular mobility $(\Delta \mathrm{K})$ can thus be kept from hitting electrode 1 by applying a low DC voltage (-20 to $20 \mathrm{~V})$, namely the compensation voltage to electrode 2 in order to draw the ion swarms away from colliding into electrode 1 . In the DMS analyzer only ions of a given $\Delta \mathrm{K}$, matching the offset distance causes by the compensation voltage, would successfully pass through the electrodes and be detected at the Faraday plate. Hence, the mobility spectrum is a plot of compensation voltage vs. signal intensity, where ions of different species are scanned within a range of compensation voltage and allowed to be detected.[17] By the late 1990's, at least two designs of high-field tubes were available. One was developed at the National Research Council in Canada based upon a concentric cylindrical geometry,[96] and another was based on a planar rectangular geometry developed in a

collaboration between Draper Laboratory and New Mexico State University.[97] The planar geometry is much more attractive for miniaturization due to the simplicity of the design for assembling the unit.

\subsection{Ion Mobility in Gases at Atmospheric Pressure}

The movement of ion swarms inside an IMS with a weak electrical field and neutral gas at atmospheric pressure is the combination of 1) the diffusion of the gaseous ion, 2) the field effect, and 3) the electrostatic interaction between the ions and the gas molecules. Ion diffusion in a supporting atmosphere (without electrical/magnetic fields, temperature gradients or Coulomb repulsion effects) is much like diffusion of gaseous molecules. Ions flow from a region of higher concentration to a region of lower concentration according to Fick's law. Diffusion continues until the ion concentration gradient becomes zero. When the ion is subjected to an electrical field, 
the motion of the ion is affected by the diffusion effect as well as the field. Ion gains kinetic energy from the electric field and accelerates along the field lines together with the superimposed diffusion movement. Hence, the ion drift velocity is given as $\mathrm{v}_{\mathrm{d}}=\mathrm{KE}$, where $\mathrm{K}$ is the mobility coefficient (like the diffusion coefficient, D), and $\mathrm{E}$ is the magnitude of the electrical field. In addition to the diffusive force and the electrical field force the ion experiences, there are electrostatic interactions between the ion and the molecule of the counter flow gas. It is the ionmolecule interactions that cause ion of different species to separate inside the drift tube. The electrostatic interactions occur because the electron cloud on the neutral drift gas molecules is polarized by the nearby ions, which induces a dipole moment in the neutral molecules. Gas molecules that have permanent dipole or quadrupole moments will also be attracted to the ions.[17] Several ion mobility models have been established to account for the overall effects of these three main forces, diffusion, electric field, and electrostatic interaction. These models are 1) the rigid sphere model, 2) the polarization limit model, and 3) the hard-core potential model.

In the rigid sphere model, the collision between an ion and a gas molecule is treated as that of rigid spheres in which the ion is equally likely to be scattered in any direction. The mean ion energy is $\left(1 / 2 \cdot \mathrm{mv}^{2}\right)$, which is a sum of the total energy incorporating the thermal energy acquired by the ion $\left(1 / 2 \cdot \mathrm{MV}^{2}\right)$, the energy gained by the ion from the electrical field $\left(1 / 2 \cdot \mathrm{mv}_{\mathrm{d}}{ }^{2}\right)$, and the energy gained from random motion of the neutral gas molecules $\left(1 / 2 \cdot \mathrm{Mv}_{\mathrm{d}}{ }^{2}\right)$. The rigid sphere model is based on consideration of the conservation of momentum and energy as the ion randomly collides with gas molecules, along with a few other assumptions. This model yields an expression of the mobility coefficient described in Equation 6. Where $e$ is the ion charge, $N$ is the drift gas density, $\mu$ is the reduced mass of the ion-neutral collision pair, $k$ is the Boltzmann constant, $T_{\text {eff }}$ is the effective temperature of the ion which is assumed to be equal to the temperature of the drift gas, and $Q_{D}$ is the collision cross section. 


$$
K=v_{d} / E=(3 e / 16 N)\left(2 \pi / \mu k T_{\text {eff }}\right)^{1 / 2}\left[1 / Q_{D}\right] \quad \text { Equation } 6
$$

The rigid sphere model defines $Q_{D}$ as equal to $\pi d^{2}$, where $d$ is the sum of the radii of the ion and neutral molecule. The mobility equation above suggests that the mobility coefficient is inversely proportional to the gas density, the collision cross section, and the square root of the reduced mass and the effective temperature.[17]

The polarization limit model is a refinement of the rigid sphere model which includes the ion-induced dipole interaction in the collisions. This interaction is a function of the polarization of the neutral gas molecule, and the ion mobility coefficient is dependent on the gas polarizability. As the temperature approaches zero Kelvin the model states that all mobility coefficients approach a common limit; namely the polarization limit $\left(K_{p o l}\right)$. The model also suggests that the mobility is essentially independent of the ion mass, which is fundamentally incorrect. Thus far, this model has not been widely used because it has been contradicted by experimental observations.

The hard-core potential model describes the ion molecule interaction as a combination between the attractive and repulsive potentials as the ion and the neutral molecule are in close proximity. The attractive forces increase ion-neutral interactions, and thus increase the resistance which leads to the decrease in the ions' mobility. Conversely, the repulsive forces do the opposite and increase the ions' mobility. This model is relatively in good agreement with the experimental observations. 


\subsubsection{IMS Gas-Phase Ion Chemistry}

The formation of ions in supporting atmosphere at ambient pressure occurs mostly in the reaction region of the IMS. The reactant ions are conventionally formed from the supporting atmosphere using a beta emitter ${ }^{63} \mathrm{Ni}$ source. The high energy electrons emitted are in the range of 0 to $67 \mathrm{keV}$ (17 keV on average) and collide with $\mathrm{N}_{2}$ or $\mathrm{O}_{2}$ in the air creating intermediate ions (Figure 14). These electrons go through a series of ion neutral reactions to form the principal positive reactant ions $\mathrm{H}^{+}\left(\mathrm{H}_{2} \mathrm{O}\right)_{n}$ and negative reactant ions $\mathrm{O}_{2}{ }^{-}\left(\mathrm{H}_{2} \mathrm{O}\right)_{n}$, where ' $\mathrm{n}$ ' ranges between 1 to 4 depending on the moisture level in the IMS. The reactant ions are then available to transfer their charge to the neutral analyte (M) to form positive and/or negative product ion clusters.[17]

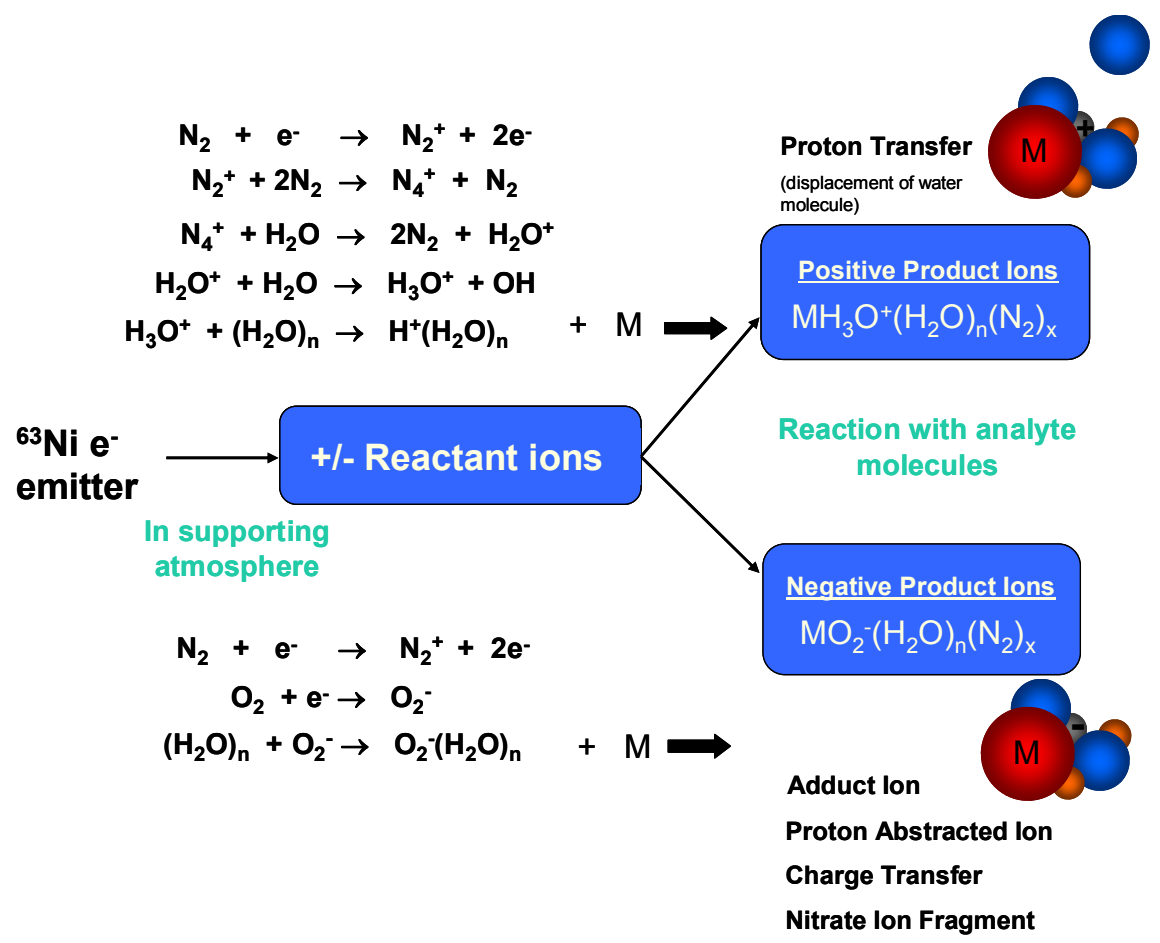

Figure 14. Formation of positive and negative product ions occur within the ${ }^{63} \mathrm{Ni}$ ionization source in supporting atmosphere at ambient pressure.

The successful formation of the positive product ion clusters depends on the proton affinity of the analyte molecule, because only analytes with higher proton affinity than the 
reactant gas can initiate the necessary reaction. In negative polarity, only analytes with higher electronegativity than the reactant gas can form negative product ion clusters. Shown in Figure 15 and Figure 16 are scales of the electronegativity [98] and the proton affinity,[17] for some selected chemical families. It is important to note that the scales given here are estimated for pure chemical compounds, which may deviate to some degree when the compounds exist in cluster form within the IMS environment. Nevertheless, these relationships provide a good guideline for choosing a dopant gas for increasing the selectivity of the IMS system.

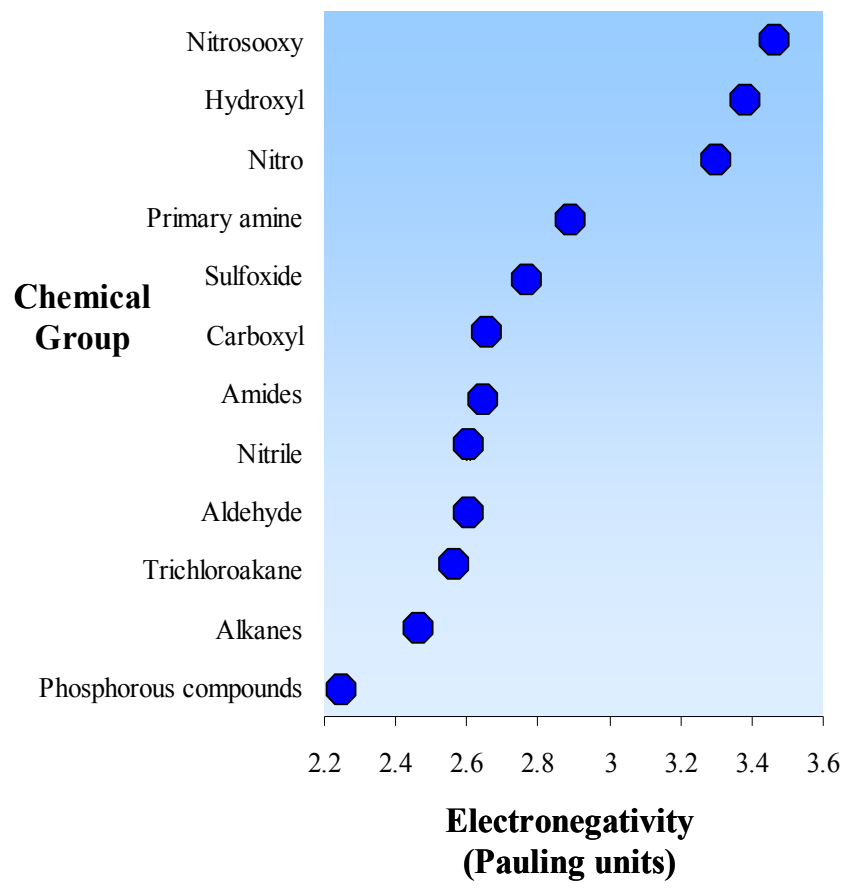

Figure 15. Electronegativity of some selected chemical classes. 


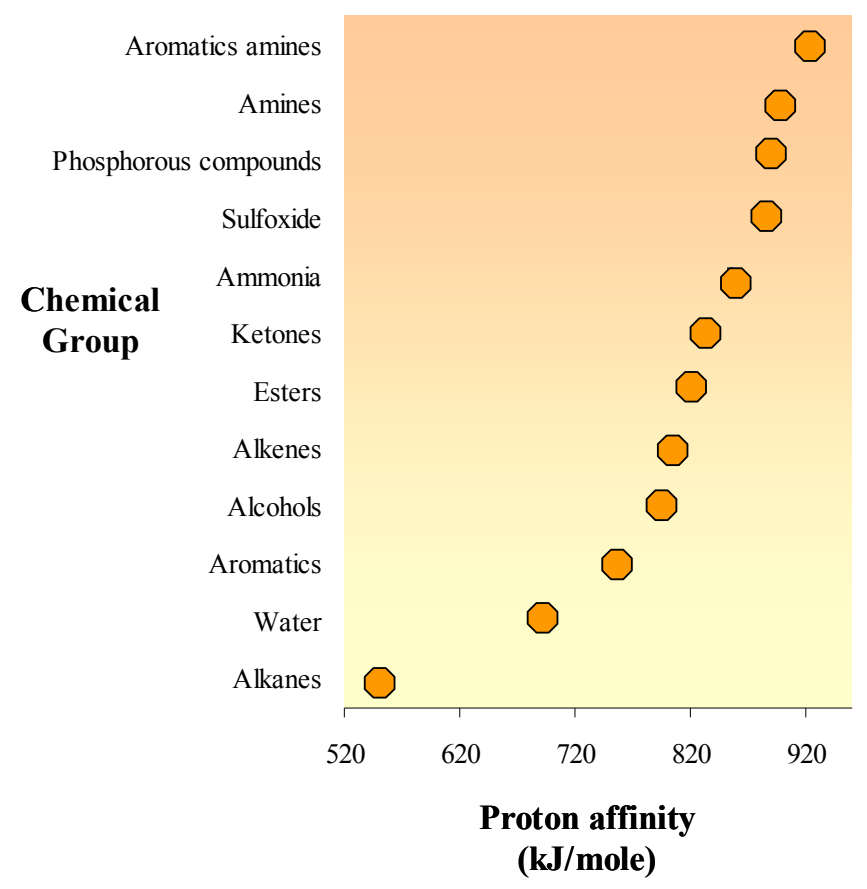

Figure 16. Proton affinity of some selected chemical classes.[17, 99]

When reactant ions are formed in clean air with trace levels of moisture, they are primarily formed as water ion clusters $\mathrm{H}^{+}\left(\mathrm{H}_{2} \mathrm{O}\right)_{n}$ and $\mathrm{O}_{2}{ }^{-}\left(\mathrm{H}_{2} \mathrm{O}\right)_{\mathrm{n}}$. The water molecule from the protonated water ion cluster $\mathrm{H}^{+}\left(\mathrm{H}_{2} \mathrm{O}\right)_{n}$ are very likely to be displaced by an analyte molecule because water molecule's proton affinity is the second lowest compared to other chemical families.[17] In air, under the right temperature and moisture conditions, most organic compounds can form positive product ions and be detected by the IMS and little selectivity is achieved. Such ease of ionization in the positive polarity makes it important to improve the IMS selectivity by doping the air inside the reaction chamber with a dopant gas (from a higher proton affinity family), so that the reactant ions created will only ionize some selective analytes of higher proton affinities rather than the reactant gas itself. In the negative polarity mode, however, the $\mathrm{O}_{2}{ }^{-}$ $\left(\mathrm{H}_{2} \mathrm{O}\right)_{n}$ reactant ion is only likely to ionize analyte molecules of higher electronegativity. Because of the dipole moment on the, $\mathrm{O}_{2}^{-}$reactant ion, neutral molecules are not likely to cluster with the 
negative ions. While increasing selectivity is not as necessary as in the positive polarity mode, dopant gas can still be used to further improve selectivity and sensitivity when IMS operates in the negative polarity mode.[17]

Once the ions are formed and have entered the drift region, they either retain their identity throughout the drift region or they undergo further chemical transformation with neutral molecules in the drift region to form ion-neutral clusters before arrival at the detector. The latter phenomenon occurs because under atmospheric pressure if moisture $\left(\mathrm{H}_{2} \mathrm{O}\right)$ is not consistently controlled and kept to the minimum inside the drift tube, or if neutral analytes are not swept away properly, they may re-enter the drift region to form clusters with the existing ions while traveling inside the drift region. Thus, the ion detected at the detector is no longer the same ion created initially in the reaction region. If such event occurred, it is apparent in the mobility spectrum both through observation of a shift in the product ion's drift time and through a sloping baseline in the mobility spectrum section between the reactant ion peak and the product ion peak. A sloping baseline indicates that some ion clusters are experiencing ion-neutral association or dissociation while inside the drift region.[100]

\subsubsection{Ion Chemistry of Explosives and Drugs}

The analysis of explosive compounds in IMS is mostly conducted in the negative polarity mode because the majority of the common explosives are from the nitro compound chemical class, one of the highest electronegativity groups. The nitro explosives form long-lived stable negative ions. Explosive product ions can be formed via one or multiple pathways (proton abstraction, charge transfer, adduct ion formation, and nitrate ion fragmentation) depending on the nature of the explosive and the reactant ions present. Proton abstraction is less common, but it 
has been reported for explosive TNT and PETN, where the $\mathrm{O}_{2}{ }^{-}$reactant ion takes a proton from the explosive compound, leaving the explosive in the form of (TNT-H) ${ }^{-}$ions. Charge transfer from the reactant ion to the explosive compound is usually followed by dissociation to form more stable fragment ion. An adduct ion is formed by the addition of a dopant gas such as dichloromethane,[35] which forms a well-defined chloride reactant ion that reacts with the analyte molecule (such as black gunpowder, RDX, NG, PETN) to form a chloride adduct ion $\left(\mathrm{M} \cdot \mathrm{Cl}^{-}\right)$. In the nitrate ion fragment pathway, nitro explosives such as EGMN and EGDN dissociate through charge transfer to form $\mathrm{NO}_{3}{ }^{-}$fragment ions and therefore are detectable. In other cases, a nitrate ion fragment may also re-associate with another analyte molecule to form a $\left(\mathrm{M} \cdot \mathrm{NO}_{3}^{-}\right)$adduct ion. This type of re-association occurs in NG, RDX, and PETN explosives. Table 12 lists the reduced mobility values for the nitro explosives and some improvised explosives found in the literature. The non-nitro explosives TATP[101, 102] and HMTD[102] are detected in positive polarity mode because of their lack of a high electronegativity functional group.[17]

Detection of illicit drugs is conducted in positive polarity mode because these compounds are more likely to form ion clusters with the hydrated proton $\left(\mathrm{H}^{+}\right)$than with the hydrated $\mathrm{O}_{2}^{-}$ reactant ion present in negative polarity mode. Some of the common illicit drugs with very high proton affinity, as shown above in Figure 16, are those that contain nitrogen in the form of amines (amphetamine, methamphetamine, MDA, MDMA, morphine, heroin etc.). High proton affinity makes them more likely to form the stable positive ion cluster $\mathrm{MH}^{+}\left(\mathrm{H}_{2} \mathrm{O}\right)_{\mathrm{n}-1}$, by displacing one water molecule from the hydrated proton reactant ion. Ammonia or nicotinamide gas can be doped to the reacting air to from $\mathrm{NH}_{4}^{+}$or $\left(\mathrm{C}_{6} \mathrm{H}_{6} \mathrm{~N}_{2} \mathrm{O}\right) \mathrm{H}^{+}$reactant ion respectively, in order to increase the selectivity of positive product ion formation. Only drugs with higher proton affinity than the reactant gas would be able to displace the $\mathrm{NH}_{3}$ or the $\left(\mathrm{C}_{6} \mathrm{H}_{6} \mathrm{~N}_{2} \mathrm{O}\right)$ molecules to form 
$\left(\mathrm{MH}^{+}\right)$. Cocaine and heroin have also been reported to from molecular ion $\left(\mathrm{M}^{+}\right)$as well as fragment ion $(\mathrm{M}-\mathrm{F})^{+}$. Listed in Table 12 are the reduced mobility values reported for some of the illicit drugs and control substances with nicotinamide dopant.[17]

Table 12. Reduced mobility values of some common explosives (detected in negative mode) and drugs (detected in positive mode).[17]

\begin{tabular}{|llll|}
\multicolumn{1}{|c}{ Explosives } & \multicolumn{1}{c}{$\mathbf{K}_{0}\left(\mathrm{~cm}^{2} \cdot \mathbf{V}^{-1} \cdot \mathrm{s}^{-1}\right)$} & \multicolumn{1}{c}{ Drugs } & \multicolumn{1}{c}{$\mathbf{K}_{\mathbf{0}}\left(\mathrm{cm}^{2} \cdot \mathbf{V}^{-1} \cdot \mathrm{s}^{-1}\right)$} \\
\hline MNT & $1.74,1.81,2.40$ & Amphetamine & 1.66 \\
2,4-DNT & $1.68,2.10$ & Butabarbital & $1.28,1.35$ \\
3,4-DNT & 1.54 & Cannabinol & 1.06 \\
$2,6-D N T$ & 1.67 & Cocaine & 1.16 \\
TNT & $1.45,1.49,1.54,1.59$ & Codeine & $1.18,1.21$ \\
Dynamite & $2.10,2.48$ & Diazepam & 1.21 \\
Nitroglycerine & $1.32,1.34,1.28$ & Heroin & $1.04,1.14$ \\
EGMN & 2.46 & LSD & 1.085 \\
EGDN & 2.46 & Methamphetamine & 1.63 \\
HMX & $1.30,1.25$ & MDA & 1.49 \\
RDX (dimer) & $1.48,1.39,1.31,(0.95)$ & MDMA & 1.47 \\
PETN & $1.48,1.21,1.15,1.10$ & Morphine & $1.22,1.26$ \\
Tetryl & $1.45,1.62$ & Opium & 1.55 \\
Comp B & $1.57,1.70,1.81$ & PCP & 1.27 \\
TATP* & 1.36 & Phenobarbital & 1.44 \\
HMTD* & 1.50 & THC & 1.05 \\
Black gunpowder & 1.88 & Thelaine & 1.14 \\
\hline
\end{tabular}

(*) Explosives detect in positive mode.

\subsection{Operational Parameters Affecting IMS performance}

Gas-ion chemistry under ambient pressure can be complicated in an uncontrolled environment. Temperature, moisture, pressure, electric field, gas flow rates, drift gas 
composition, and ion density are the essential factors in governing ion's identity, drift time, peak intensity, and resolving power. Reproducibility of the qualitative and quantitative response is achievable only when these parameters are controlled.[17] Optimizing these parameters is critical for the detection of new compounds of interest since some of their effects are compound dependent.

\subsubsection{Temperature}

Temperature has two major effects on the ion at fixed moisture level. At elevated temperatures, depending on individual analytes, dissociation of water molecules from the product ion cluster and fragmentation of the core product ions may occur to form smaller ions with faster mobility. Thus, the product ion detected at room temperature may not be the same ion detected at high temperature even for the same analyte. In addition, proton-bound dimer and proton-bound trimer (formed under high concentrations of analyte) of most compounds dissociate back to the monomer ion form if temperature is above 80 to $100{ }^{\circ} \mathrm{C}$ and -20 to $0{ }^{\circ} \mathrm{C}$, respectively.[100, 103] Hence their identities are never retained when an IMS analyzer is operated at elevated temperatures. Moreover, if the temperature of the IMS drift tube is not homogenous, a change of identity in product ion can occur while the ion swarms travel down the drift region. Hence, for valid comparison of mobility spectra between laboratories, it is critical to report the correct measured temperature of the IMS.

\subsubsection{Moisture}

Moisture has a large effect on the identity of the product ions because, depending on the moisture level inside the drift tube, the product ions may cluster with more than one water molecule or decluster the water molecules changing their effective masses and collision cross sections. Consequently, the ions' identity and drift time are changed. If moisture is reduced to a 
level below $10 \mathrm{ppm}$ the reactant ions formed in air may no longer be the hydrated protons, $\mathrm{H}^{+}\left(\mathrm{H}_{2} \mathrm{O}\right)_{\mathrm{n}}$. Instead other ions such as $\mathrm{H}_{2} \mathrm{O}^{+}, \mathrm{N}_{4}{ }^{+}$, and $\mathrm{N}_{2}{ }^{+}$are formed. As the result, ionization of neutral analytes will occur through charge transfer and dissociative charge transfer mechanisms rather than proton-transfer. $[17,103]$ Multiple reactant ion species may spread the response of a given analyte when compared to only one type of reactant ion. When the moisture level inside the drift tube drops to less than $1 \mathrm{ppm}$, regardless of the temperature, most analytes will form fragment ions.[104]

\subsubsection{Pressure}

Pressure has been shown to have an effect on peak resolving power and peak to peak resolution. However, there is no effect on the separation factors because the ion peaks shift linearly with pressure. Tabrizchi et al. shown that the resolving power and resolution decrease as pressure within the drift region decreases. The decrease in resolving power and resolution is most

likely the result of the increase in ion density at low pressure. Compensating for low pressure by decreasing the gate width and allowing fewer ion into the drift region has been shown to restore resolving power and resolution.[105]

\subsubsection{Electrical field gradient}

The electrical field within the IMS drift tube is usually optimized to achieve the best ion transport to the detector for improved ion peak intensity and resolving power. Inside the drift tube, there are four regions which have different electrical fields; 1) the field between the source and the first set of wires on the ion shutter $\left.\left(\mathrm{E}_{\mathrm{s}-\mathrm{g}}\right), 2\right)$ the field between the 2 alternating set of wires at the ion shutter $\left.\left(E_{g}\right), 3\right)$ the field between the ion shutter and the aperture grid, which is the drift 
field $\left(E_{d}\right)$, and 4) the field between the aperture grid and the detector $\left(E_{a-d}\right)$. Previous studies showed the effect of each of the above electrical fields on the IMS performance parameters such as, peak intensity, peak FWHM, baseline level, and drift time. The optimal values for each of these electrical fields vary from one drift tube to another.[94]

As the $\mathrm{E}_{\mathrm{s}-\mathrm{g}}$ increases, the ion swarms are directed out of the source region more successfully and an improvement in peak intensity is observed. Peak intensity reaches a plateau after the $\mathrm{E}_{\mathrm{s}-\mathrm{g}}$ exceeds $\sim 400 \mathrm{~V} \cdot \mathrm{cm}^{-1}$ (or 2 times the drift field).[94] In the traditional low-field IMS, the mobility coefficient $\left(\mathrm{K}_{0}\right)$ is considered to be independent of the applied drift electric field when the field is kept under a certain value, commonly at $300 \mathrm{~V} \cdot \mathrm{cm}^{-1}$ or below because at low field, the energy acquired by the ion from the electric field is considered negligible due to constant ion-gas molecule collisions. A stronger drift field $\mathrm{E}_{\mathrm{d}}$ usually results in higher peak intensity, smaller FWHM, and shorter drift time but as the field exceeds an upper limit, $\mathrm{K}_{0}$ will no longer be constant and will instead become dependent on the drift electric field. While it has been reported that IMS resolving power increases with the square root of the voltage applied for the drift field, in reality there exists an optimum voltage above which resolving power also decreases. This optimum voltage was suggested to be both compound and initial gate width dependent.[94]

If the drift field is strengthened and the gate field is not re-adjusted, then the baseline of the spectrum would rise because ions are allowed to leak through the ion shutter even when it is supposed to be closed. This leakage increases as the drift field increasingly penetrates the ion shutter region. Similarly, if the gate field $\left(E_{g}\right)$ is not kept at an optimal level with respect to the drift field, ions would penetrate causing an increase in the baseline.[94] 
Finally, the field between the aperture grid and the detector $\left(\mathrm{E}_{\mathrm{a}-\mathrm{d}}\right)$ has been shown to be optimal when at 2 to 3 times stronger than the drift field, but also has been shown at 10 times in other studies. If the field is lower than the optimal range, distortions in ion peaks are observed, and ions will be more likely to collide at the aperture grid rather than the detector.[94]

Field homogeneity and linearity is desired along the drift region of the drift tube because ions are then focused along the central axis with minimum loss to the drift wall during the separation process. Baumbach showed improvement in peak intensity and peak resolving power when the conductive drift rings' thickness is reduced to minimize the plateau section of the drift field in order to create a more linear and homogenous field gradient inside the drift region.[106]

\subsubsection{Gas flows}

The sample gas is introduced into the drift tube as a carrier gas to deliver the analyte to the ionization source as well as to provide the supporting atmosphere for ionization to occur within the ${ }^{63} \mathrm{Ni}$ ionization source. The drift gas is introduced to provide the environment for the ion swarm separation process. The gas flow rates and orientation must be carefully modeled and balanced to achieve optimal ion signal response. The sample flow rate and the drift gas flow rate are usually set at $500 \mathrm{~mL} \cdot \mathrm{min}^{-1}$ and $200 \mathrm{~mL} \cdot \mathrm{min}^{-1}$, respectively.[17, 107] It is critical not to have the sample gas carrying neutral analytes enter the drift region because the presence of neutral analyte molecules may form clusters with existing product ion swarms, changing their identity.

There are several methods through which the sample gas flow and the drift gas flow are introduced into the IMS drift tube. The most common method is to introduce the sample gas at the inlet of the drift tube, and drift gas at the end of the drift tube where the detector sits. These 
two gases are then meet and exit at the same outlet, which is located between the ionization source and the ion shutter. In other IMS designs, the outlet for these gases is located at the front of the drift tube, and sample gas is introduced at the side of the drift tube at a point after the ionization source.[17] It is also possible to have the sample gas exit at a different outlet than the drift gas, so that the two gases are never mixed.

\subsubsection{Drift gas composition}

Thus far, the parameters described above (temperature, moisture, electric field strength, and gas flow rates) effect all ion species in the same way or in relatively similar manner. Drift gas composition, on the other hand, causes different effects for different ion species under otherwise identical conditions. Interaction of the drift gas with individual ion species depends on the drift gas reduced mass, the drift gas polarizability, and the ion-gas collision cross section. When changing the drift gas from air to argon, the arrival of one ion species may speed up while a second ion species is delayed. The non-relative change makes it possible to resolve overlapped ion species that cannot otherwise be resolved under a particular drift gas environment.[17, 108]

\subsubsection{Ion density}

Achieving maximum ion response or low detection limits is always the goal of an analytical technique. Increasing the production of ion and the gate width usually results in higher sensitivity. However, when the ion density within the IMS exceeds a certain range, spatial dispersion occurs rapidly due to Coulomb repulsion. Mariano et al. reported the upper limit of ion density to be $\sim 19 \mathrm{pC} \cdot \mathrm{cm}^{-2},[109]$ and Tolmachev et. al. reported an upper limit when the ion population is greater than 10,000 elementary charges.[110] With excess ion density, Coulomb 
repulsion causes ion swarms to spread out, and ions are lost along the drift wall before they get a chance to reach the detector. The spread of ions leads to a decrease in signal response and poor resolving power. Restricting the ion density is more critical for the design and operation of miniaturized IMS instruments because the small drift tube limits the volume within which the optimal number of ion needs to reach the detector in order to provide a good response.

\subsection{IMS Limit of detection, Linear Dynamic Range, and \% RSD}

Despite the low duty cycle of the conventional BN-gate, which only allows $1 \%$ of the total ion created to be transported into the drift region, IMS detection limits for most compounds are still in the low nanogram range, and some are as low as the picogram level. As a trace analyzer, IMS has excellent detection limits, second only to fluorescence and conjugated polymer sensor technologies.[16] IMS is known to have a smaller linear dynamic range compared to other analytical techniques. A common IMS response curve for a compound ionized by a ${ }^{63} \mathrm{Ni}$ source shows the first linear dynamic range is between 10 to $1000 \mathrm{ng}$. At higher mass ranges, there exist a second and then a third dynamic range which are between 1000 to $2000 \mathrm{ng}$, and above $2000 \mathrm{ng}$, respectively. The multiple dynamic ranges behavior in IMS is explained in literature as being the result of the rate of reactant ion consumption being higher than the rate of reactant ion formation from the ${ }^{63} \mathrm{Ni}$ source. When an IMS instrument is operated under a controlled laboratory environment, the percent relative standard deviation (\% RSD) of signal intensity ranges between $2 \%$ to $25 \%$ for most compounds and decreases as the sample mass increases. However, the signal \% RSD may rise rapidly when changing location and environment in which the analysis takes place because of change in pressure, moisture, etc. 


\subsection{Limitations and Future Developments of IMS}

IMS is a niche technology serving critical needs in specific applications, such as the detection of illicit substances and volatile chemical components from environmental samples, etc. However, IMS is unlikely to be used the way mass spectrometry (MS) is utilized today to characterize unknown samples. A mass spectrum can be interpreted to match a particular ion fragmentation pattern to the molecular structure of a sample molecule, but the spectrum generated by IMS cannot provide such information. Thus far, there are no models that can link ion mobility to an ion's structure.[17] Therefore IMS are not yet utilized for general applications, but only for specific applications where mobility of a known ion has been programmed in the detection channels. Limited studies have shown the classification of mobility spectra by functional groups using information gained from fragment ion swarms and neural networks.[100, 104, 111] Further development in this area may further advance the capability of IMS technology for general applications. Finally, there is a strong need for the IMS community to agree on the use of standards, formalized spectral libraries, and unified operational practices so that results can be qualitatively and quantitatively compared between instruments and laboratories. 


\section{CHAPTER 8: COMPUTER SIMULATION FOR THE DESIGN OF ION MOBILITY SPECTROMETRY}

\section{CONTENT}

PAGE

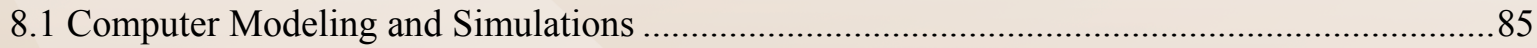

8.2 Challenges of Modeling Ion in Gas at Atmospheric Pressure …............................................86

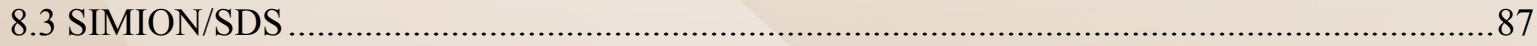
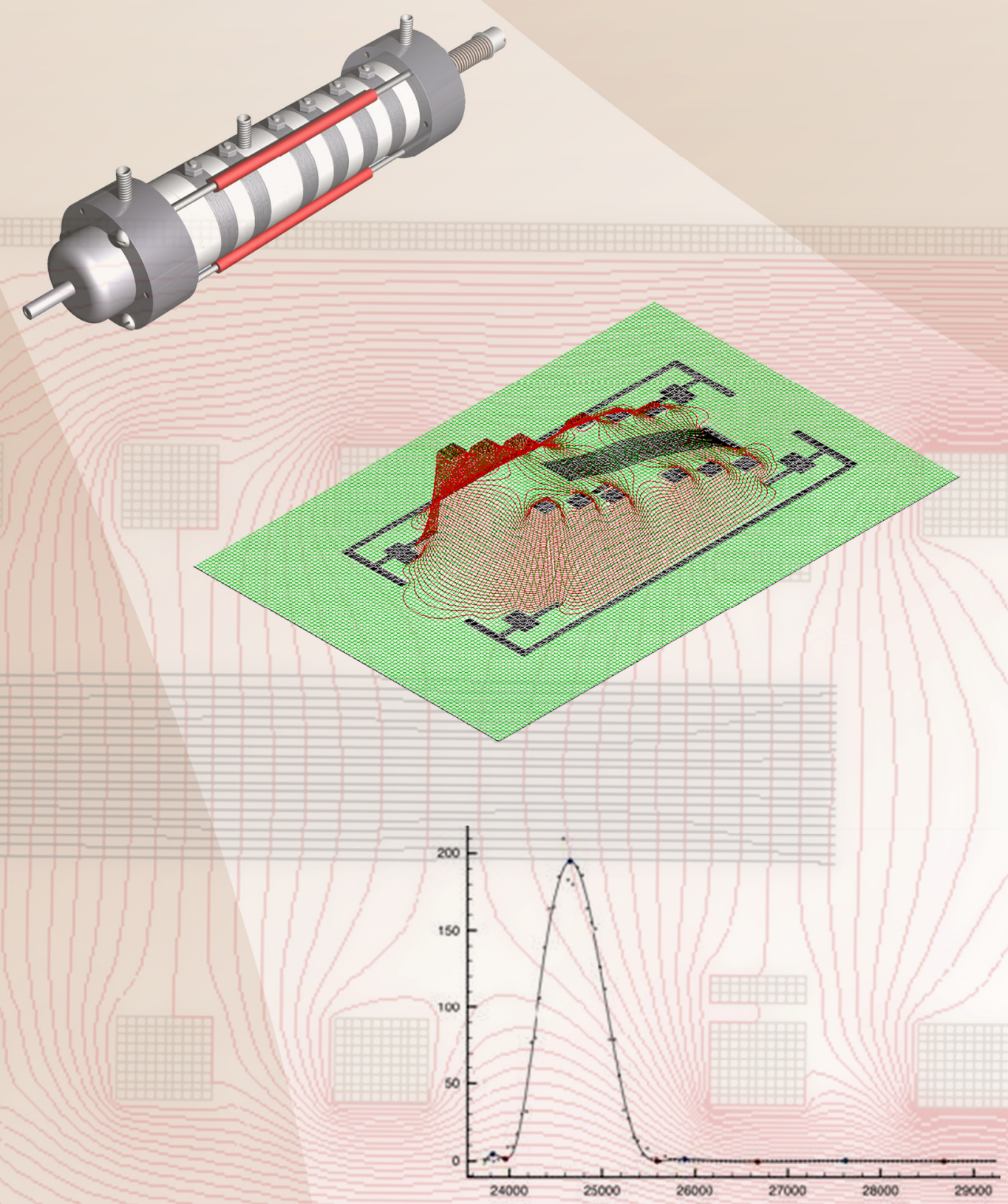


\subsection{Computer Modeling and Simulations}

Previously, the only computer modeling of ion mobility spectrometry (IMS) instruments has been either to evaluate the electrostatic fields of different drift tube designs $[94,106,112]$ or to simulate the drift gas and carrier gas flow characteristics within the drift tube.[113] As stated by Baumbach and coworkers, an evolutional simulation approach is needed in order to model ions in electrostatic fields within the IMS taking into account the flow conditions of the neutral gaseous molecules.[113] SIMION [114-116] revolutionized the ability to model instruments analyzing gas-phase ions in a vacuum, especially mass spectrometers.[117, 118] The recent introduction of the statistical diffusion simulation (SDS) user program for SIMION [119] for modeling ion trajectories in viscous (i.e., atmospheric pressure) regimes in electrostatic and magnetic fields [115] has opened the door to model IMS instruments. In 2005, Sandia National Laboratory reported the use of SIMION to simulate transportation of ion swarms through an IMS/MS interface.[120] The present study incorporated an external programming code that compiled within SIMION to take into account the collision effect of neutrals on the ion swarms; however, this programming did not account for the effect of diffusion, which is important in IMS as well as for IMS/MS interfaces.[120, 121]

Ion formation, separation, and detection are dictated by instrument operating parameters such as the type of ionization source, temperatures in various zones (e.g., drift tube), dopant gas type, carrier and drift gas compositions and flow rates, imposed electrical field, gate mechanism and timing, as well as the physical geometry of the drift tube.[17, 94, 122, 123] These parameters affect the overall selectivity, sensitivity, and peak resolving power of the instrument and can be optimized for a given analyte or class of compounds of interest.[30] Of the above listed parameters, drift tube temperature, gas flow rates, gas compositions, and gate width (i.e., duration 
the gate is open for ion passage) are readily changeable in commercial IMS instruments, while others are usually fixed by the manufacturers.

\subsection{Challenges of Modeling Ion in Gas at Atmospheric Pressure}

Unlike mass spectrometry, IMS operates at elevated and atmospheric pressures, and separates ions based on size and/or shape as opposed to mass. IMS was originally referred to as plasma chromatography [70, 123-126] because ions of the chemical analytes are characterized using gas-phase ion mobility determined by the arrival times of the ion clouds.[17, 126, 127] The separation between ions of different sizes and/or shapes occurs while traveling along an electrostatic gradient in a drift tube at atmospheric pressure. $[17,125,126]$ The thermal velocity of an ion in an IMS instrument is well over $100 \mathrm{~m} \cdot \mathrm{s}^{-1}$ because it is being bombarded by millions of collisions per second with the neutral gas molecules due to the gas kinetics at standard atmospheric conditions. These collisions create a sort of drunkard's walk (i.e., Brownian motion) that results in diffusion. The effect of the buffer gas reduced mass $(\mu)$ and collision cross section $\left(\Omega_{D}\right)$ is easily seen in

Equation 7: Where $(e)$ is the charge of an electron; $(\mathrm{N})$ is the number density of buffer gas molecules; $(\alpha)$ is the correction factor; $\left(\mathrm{T}_{\text {eff }}\right)$ is the effective temperature. $[17,126]$

$$
\mathrm{K}=\frac{3 e(2 \pi)^{1 / 2}(1+\alpha)}{16 \mathrm{~N}\left(\mu k \mathrm{~T}_{\text {eff }}\right)^{1 / 2} \Omega_{D}\left(\mathrm{~T}_{\text {eff }}\right)}
$$

Equation 7

To correctly model the ion trajectories inside an IMS instrument, the SDS user program must be incorporated into the SIMION ion optics modeling program to account for both the mobility and the longitudinal diffusion [123] terms which exist at atmospheric pressure.[120, 
121] A detailed description of the SDS algorithms and how they are integrated into the ion trajectory calculation in SIMION has been published previously.[119] Briefly, SDS treats viscous drift motion and diffusion as separate phenomena, even though they are not independent in reality. Viscous effects are treated with a relatively straightforward Stoke's Law model, while diffusion is emulated using a collection of tabulated collision statistics and randomized ion jumping to provide an unbiased approximation of diffusion over a wide range of ion mass to collision gas mass ratios.[119, 121]

\subsection{SIMION/SDS}

SIMION/SDS has been used to model ion behavior in viscous environments for comparison with ion motion in vacuum related to electrostatic refraction, influence of wire grids and electric fields, as well as magnetic fields and charge repulsion.[121] However, no attempt has been made to evaluate the predictive power of SIMION/SDS in simulating ion trajectories inside a complete IMS instrument nor has there been a comparison against experimental data for molecular ion of interest, such as 2,4,6-trinitrotoluene and cocaine. In this dissertation, SIMION/SDS was utilized to create simulation method for ion in the gas phase in a complete IMS, and presents insights into simulation of an actual commercial IMS, the challenges of using SIMION/SDS, and the comparison of the model with experimental data under various IMS instrumental conditions. The operating parameters studied were drift:carrier gas flow rates, drift gas composition, and ion gate width. It should also be noted, however, that incorporating the effect of chemical reactions and their effect on IMS spectra is beyond the capabilities of the SIMION/SDS program. 


\section{PART III: MATERIALS AND METHODS}

$\begin{array}{ll}\text { CONTENT } & \text { PAGE }\end{array}$

CHAPTER 9: Method Development for Detection of Drugs and Explosives ..............................89

CHAPTER 10: Method Development for IMS Modeling and Simulations................................106

CHAPTER 11: Construction of an in-house Designed IMS .................................................117 


\subsection{Instrumentation}

A General Electric Ion Track (Wilmington, MA) Itemiser 2 Ion Mobility Spectrometer was used to detect the compounds of interest. The GE Itemiser 2 front end was coupled with a SPME interface designed by the Almirall research group (see Figure 17). Details on the IMSSPME set up and the SPME interface design have been reported elsewhere.[27] A Smiths Detection IonScan (Mississauga, ON, Canada) 400B Ion Mobility Spectrometer was used to determine the reduced mobilities for the compounds detected.

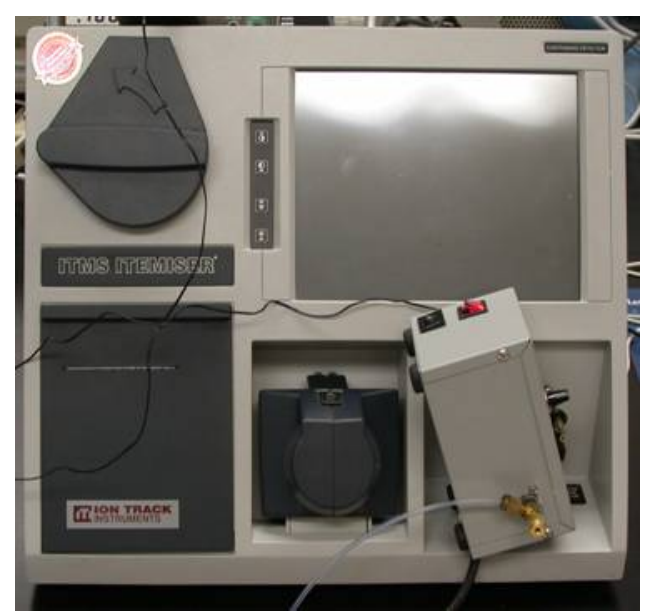

Figure 17. SPME-IMS interface and GE Itemizer 2.

\subsection{IMS Optimization for New Compounds of Interest}

The current operating conditions of IMS devices are optimized by manufacturers for a list of compounds (listed in Figure 18) which do not include the volatile signature compounds mentioned above. Thus, new instrumental parameters were necessary in order to detect these compounds. 


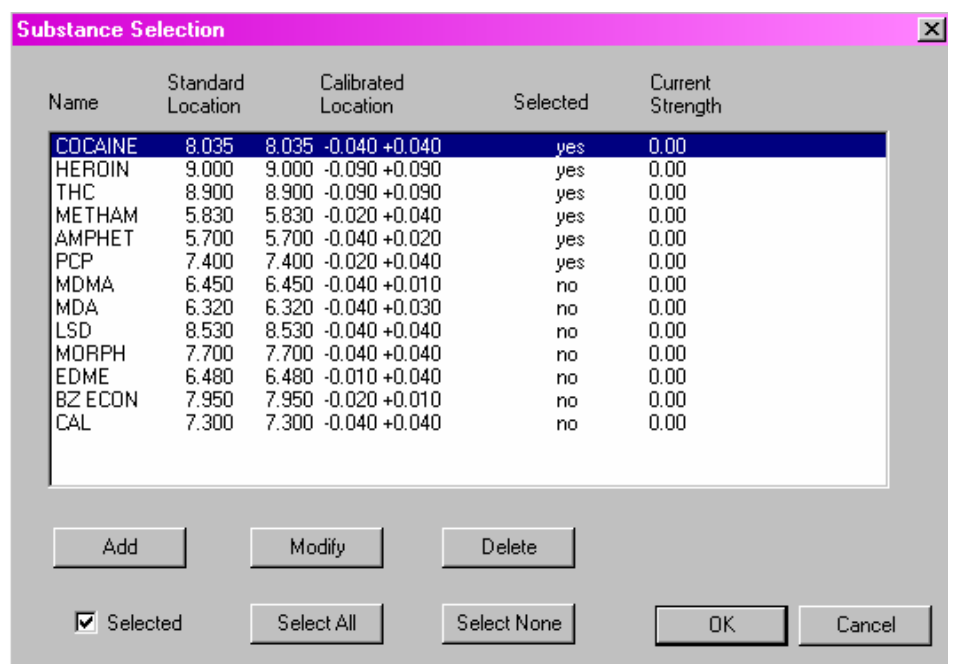

Figure 18. Substance window in nartcotic mode (GE Itemiser 2).

When performing systematic optimization, it is important to choose an appropriate optimization algorithm that is compatible with the analytical system utilized. Since variations in the operating conditions (with several variables having significant effects) can affect the final response of the IMS system in a non-linear manner, industry standard algorithms that depend on continuous (or linear) objective functions are not suitable for finding the true globally optimal conditions. A genetic algorithm (GA) was selected as the most suitable method of optimization for IMS instruments for the purposes of this study.

\subsubsection{Systematic Optimization of IMS instrumental parameters}

Genetic algorithms (GA) are a particular class of evolutionary algorithm that use techniques inspired by evolutionary biology to generate the next generation of the test population (set of configurations) based on the traits and performance of the previous population [128] as shown in Figure 19. 


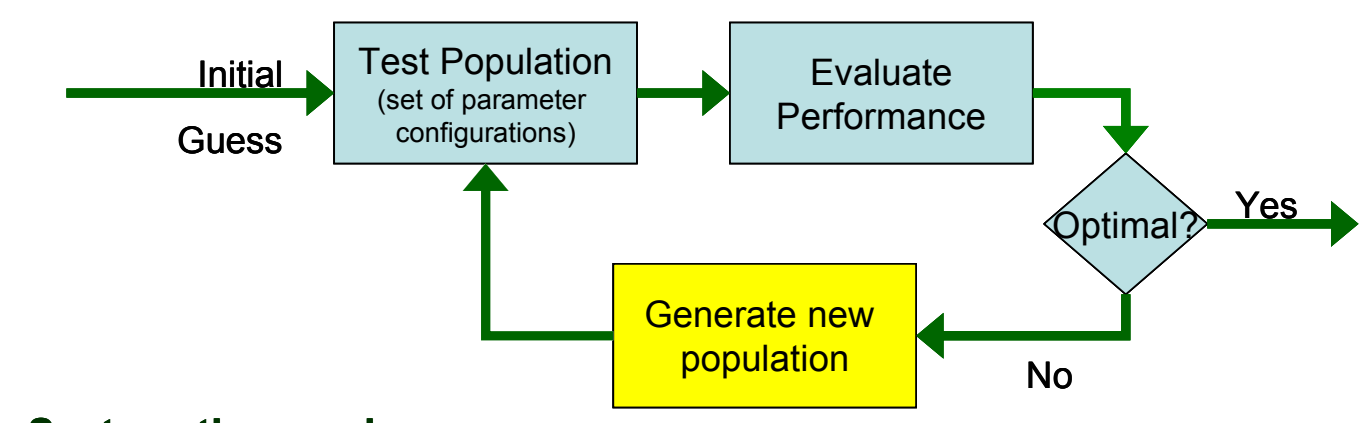

Systematic search:

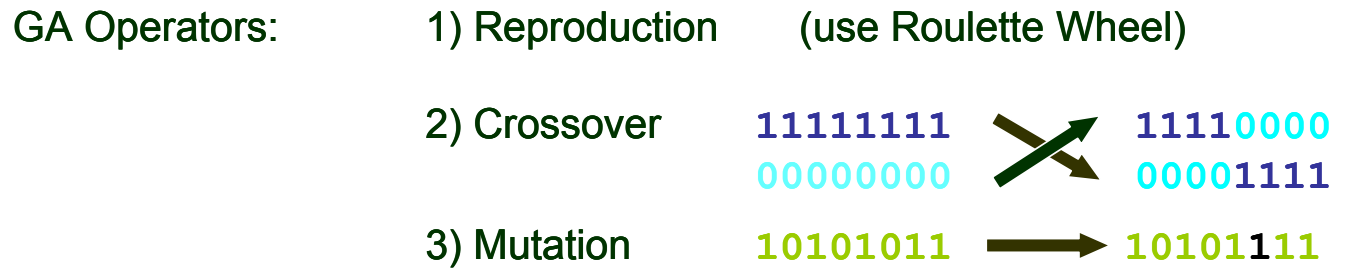

Figure 19. Schematic of optimization using a Genetic Algorithm.

The best performing configurations can bias the next test population towards even better results.[128-130] Three genetic algorithm operators, reproduction, crossover, and mutation, are used in the computation code to create the next generation. Reproduction operates according to the fitness of the members (in this case, the intensity value) such that only the fittest members will be selected into the mating pool. The crossover operator selects any two members from the mating pool and randomly exchanges some portion of the genetic codes to create two new offspring (new configurations). Finally, the mutation operator alters one or more genes on the new offspring with a probability equals to the randomized mutation rate.[128]

\subsubsection{Genetic Algorithms (GA) Procedure}

There are 8 steps in the process of performing GA operation on an IMS system:

1. Identify significant variables in the IMS system. 
2. Define the operating ranges for each.

3. Assign each of the settings a genetic code represented by binary numbers.

4. Create the first generation of the population using a random generator, then determine the IMS response (in this case, the intensity value).

5. Use the information from the first generation to produce the next generation off-spring.

6. Determine the off-spring's response in the IMS, then add them into the new population.

7. Repeat steps $5-7$ until the average responses of the newer generations have converged.

8. Report the highest response member as the "current optimum condition" after implementation of $\mathrm{n}$ generations.

\subsubsection{GA Binary Number Representation}

Table 13 reports the instrumental parameters optimized in this study. The range and increments for each of the parameters were decided prior to the optimization experiment and each setting was coded using binary numbers. For example, the operating mode has two settings, therefore could be represented with 1 bit. A " 0 " is for negative ionization mode, and a " 1 " is for positive ionization mode. The reagent gas has 4 possible settings ( 4 reagent gases), therefore could be represented with 2 bits such that " 00 " for Air, " 01 " for nicotinamide, " 10 " for ammonia, and " 11 " for $\mathrm{CH}_{2} \mathrm{Cl}_{2}$. Once all IMS parameters and settings were coded, they were combined in series to represent a particular instrument configuration, such as 01001011101 . This representation of the parameter settings is understood by genetic algorithm (GA) operators. 
Table 13. Experimental setup and encoding of IMS settings for binary representation.

\begin{tabular}{|l|lc|}
\hline IMS Parameters & \multicolumn{1}{c|}{ Range, Increment } & \# of settings \\
\hline Temperature & $\begin{array}{l}40 \text { to } 190{ }^{\circ} \mathrm{C}, \\
\text { increments of } 10^{\circ} \mathrm{C}\end{array}$ & 16 \\
\hline Detector Flow & $\begin{array}{l}50 \mathrm{~mL} \cdot \mathrm{min}^{-1} \text { and } 350 \mathrm{~mL} \cdot \mathrm{min}^{-1}, \\
\text { increments of } 1000 \mathrm{~mL} \cdot \mathrm{min}^{-1}\end{array}$ & 4 \\
\hline Sample Flow & $\begin{array}{l}500 \mathrm{~mL} \cdot \mathrm{min}^{-1} \text { and } 3500 \mathrm{~mL} \cdot \mathrm{min}^{-1}, \\
\text { increments of } 1000 \mathrm{~mL} \cdot \mathrm{min}^{-1}\end{array}$ & 4 \\
\hline Reagent Gas & Air, Nicotinamide, Ammonia, $\mathrm{CH}_{2} \mathrm{Cl}_{2}$ & 4 \\
\hline Operating Mode & Negative or Positive Ionization & 2 \\
\hline
\end{tabular}

\subsubsection{GA Convergent at Optimal Operating Conditions}

GA optimization was considered to have converged when there was no longer a significant difference between the average responses of three consecutive generations. In the first generation, the population had 20 random members, and the subsequent generations each had 20 members of which 10 members were new off-spring. The average and the maximum response of each of the generations were plotted to demonstrate the convergence of GA for a model compound DMNB after evaluating 6 GA generations.

\subsubsection{Comparison of $G A$ to Other Optimization Techniques}

To demonstrate the advantage of using this GA optimization technique, it was compared to conventional and random optimization techniques. The performance (intensity) of the 80 members from 6 GA generations were recorded along with 80 new random members generated from using random function in macro excel. The 80 members from each method were plotted for comparison of the members' intensity. Another well known optimization technique tested was SIMPLEX. The SIMPLEX optimization method is very sensitive to the investigator's choice of starting point, and so it is more suitable for performing the fine tuning step in the optimization 
process rather than as a stand alone technique. This study evaluated 10 members of SIMPLEX using the GA's best configuration as SIMPLEX's reference starting point.

\subsection{Chemicals and Drug Samples}

The volatile signature compounds piperonal and methyl benzoate were obtained from Aldrich Chemical Company, Inc. (Milwaukee, WI). (1S)-(-)-alpha-Pinene, (1S)-(-)-beta-Pinene, and limonene were obtained from Acros Organics (New Jersey). Dynacal Permeation devices containing ammonia, and methylene chloride used as dopants were obtained from VICI Metronics Inc. (Poulsbo, WA) with a permeation rate of 3,100 $\mathrm{ng} \cdot \mathrm{min}^{-1}+/-10 \%$ at $30^{\circ} \mathrm{C}$ and 460 ng. $\min ^{-1}+/-15 \%$ at $30{ }^{\circ} \mathrm{C}$, respectively. The other dopants nicotinamide (permeation rate of 1495 $\mathrm{ng} \cdot \mathrm{min}^{-1}+/-10 \%$ ) and methylene bromide were obtained from Acros Organics (New Jersey).

The IMS analyses of cocaine $\mathrm{HCl}$ and free base, MDMA tablets, and marijuana (including hydroponically grown) samples were performed at the Miami-Dade Police Department Crime Laboratory Bureau. These drugs were typical exhibits obtained from evidence seized in drug cases within Miami-Dade County. Cocaine and marijuana samples were contained inside fitted Ziploc bags, while MDMA tablets were placed in aluminum weighing boats and then placed inside quart cans. The household consumable items used in the interference studies such as rosemary, oregano, sesame seeds, and black pepper were purchased from a local grocery market. Green tea leaves, and dried mushrooms were import products from China. A typical hemp rope was also tested for possible interference peaks. 


\subsubsection{Preparation of Drug Samples}

To generate an equilibrium headspace concentration, known amounts of the sample analytes were placed in $15 \mathrm{~mL}$ glass vials manufactured by Supelco, Bellefonte, PA. The vials were allowed to sit until equilibrium was reached prior to sampling. To check for equilibrium establishment, the headspace concentration of each vial was regularly monitored over days and weeks. Background spectra of the empty glass vials are also collected to ensure no contamination. In other cases, quart metal cans were used as container. The equilibrium headspace concentrations [conc. $]_{H S}$ of analytes in air were estimated using the ideal gas law, with pressure being the vapor pressure of the analytes $\left(P_{\text {vap }}\right)$ as shown in Equation 8. For direct injection of headspace gas into the IMS, the amount of mass $(m)$ delivered was calculated for a known volume injection $\left(V_{\text {injection }}\right)$ using Equation 9. The vapor pressure of the different analytes is given in Table 14 .

$$
\begin{array}{cc}
P_{\text {vap }} V_{H S}=n R T-->[\text { conc. }]_{H S}=\frac{n \times M W}{V_{H S}}=\frac{P_{v a p}}{R T} & \text { Equation } 8 \\
m=[\text { conc. }]_{H S} \times V_{\text {injection }} & \text { Equation } 9
\end{array}
$$

Table 14. Dominant headspace components and corresponding vapor pressures of the parent

\begin{tabular}{|c|c|c|c|}
\hline Parent Compound & Vapor Pressure (torr) & $\begin{array}{c}\text { Associated Headspace } \\
\text { Components }\end{array}$ & $\begin{array}{c}\text { Vapor Pressure } \\
\text { (torr) }\end{array}$ \\
\hline $\mathrm{RDX}$ in $\mathrm{C} 4$ & $1.1 \times 10^{-9} @ 25^{\circ} \mathrm{C}$ & $\begin{array}{l}\text { 2,3-dimethyl- } \\
\text { 2,3dinitrobutane }\end{array}$ & $2.1 \times 10^{-3} @ 25^{\circ} \mathrm{C}$ \\
\hline Cocaine & $1.2 \times 10^{-7} @ 25^{\circ} \mathrm{C}$ & Methyl Benzoate & $0.28 @ 20^{\circ} \mathrm{C}$ \\
\hline $\begin{array}{l}\text { 3,4 Methylenedioxy } \\
\text { methamphetamine }\end{array}$ & 90mmHg@70F & Piperonal & $1.0 @ 87^{\circ} \mathrm{C}$ \\
\hline THC in Marijuana & $\mathrm{N} / \mathrm{A}$ & $\begin{array}{l}\text { Alpha Pinene } \\
\text { Beta Pinene } \\
\text { Limonene }\end{array}$ & $\begin{array}{l}3.0 @ 20^{\circ} \mathrm{C} \\
2.0 @ 20^{\circ} \mathrm{C} \\
1.3 @ 20^{\circ} \mathrm{C}\end{array}$ \\
\hline
\end{tabular}
compounds of interest. 


\subsubsection{Preparation of Interference Samples}

Each sample of the 7 household consumable items contained in the quart cans weighed approximately 0.5 grams. Each set of cans was tested at three different IMS settings (cocaine, MDMA and marijuana). The extractions were performed using the same fiber chemistry described previously, and with an extraction time of 30 minutes for all cans. This work was performed to test if the volatile components from the household consumable items form product ions with drift times similar to those of the volatile components of drugs. In the mixture experiments, the analysis was performed with the drugs added to each of the household consumable items cans to examine the sampling extraction competition. Three sets of 7 household consumable items were prepared, one set for each drug. The first set of cans each contained about $0.5 \mathrm{~g}$ of household consumable item samples and $0.5 \mathrm{~g}$ of powder cocaine in separate Ziploc bags. The second set of cans each contained $0.5 \mathrm{~g}$ of household consumable item samples and $2.3 \mathrm{~g}$ (10 tablets) of MDMA. Lastly, the third set of cans each contained $0.5 \mathrm{~g}$ of household consumable item samples and $1.4 \mathrm{~g}$ of marijuana sample. Each set of cans was also extracted for 30 minutes using the same fiber chemistry. The SPME fibers containing the extracted analytes were then desorbed, and the analytes were introduced into the IMS for analysis.

\subsubsection{Preparation of MDMA for the Blind Test Study}

For the blind test study, 10 cans were prepared and the contents were only reviewed after the analysis. Some of the cans were filled with the interference commodities, some were filled with MDMA tablets alone and some with the MDMA + interferences, and some were left blank. The MDMA were fresh tablets seized by the Florida Highway Patrol (exact date is unknown) and were loaned to FIU for the study. All cans were sealed as shown in Figure 20 and were locked up overnight for next morning sampling. The cans were individually sampled with SPME fiber 
(PDMS) for a $0.5 \mathrm{hr}$ extraction time and followed by IMS analysis at the optimal instrumental setting for piperonal.

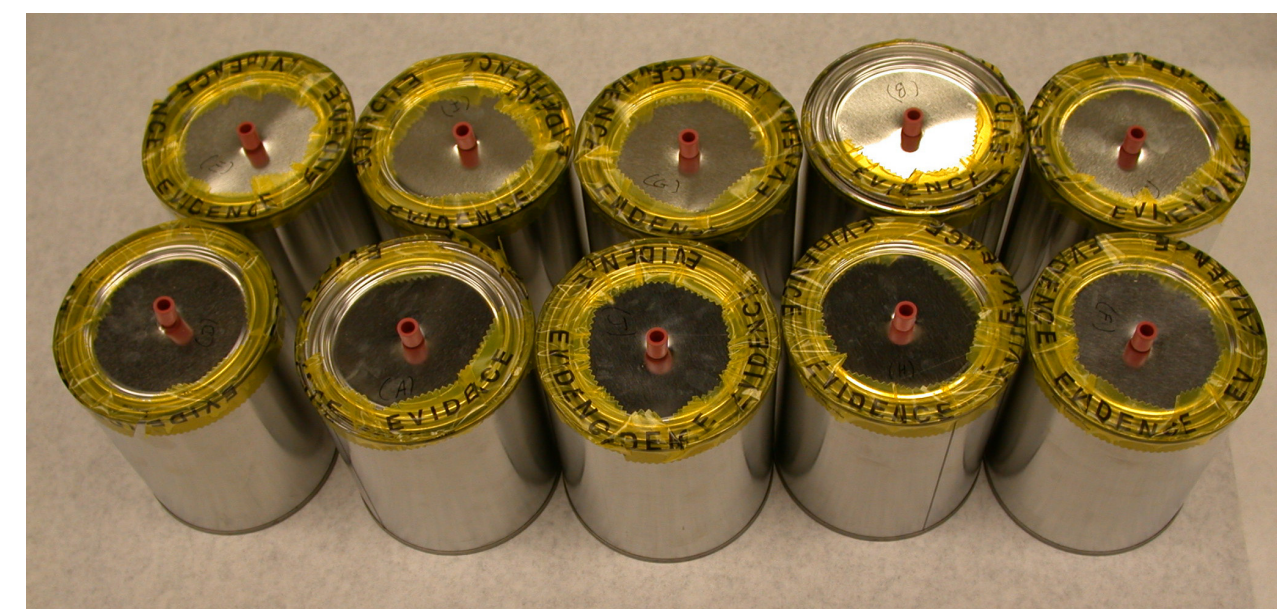

Figure 20. 10 cans sealed for MDMA blind study of which some contain MDMA as well as interference products.

\subsection{SPME Extraction for Volatile Components of Drugs}

\subsubsection{Determination of Headspace Volatile Components}

The analytes were extracted from the headspace of a $15 \mathrm{~mL}$ vial containing the sample and pre-concentrated on a PDMS SPME fiber for known time intervals. Figure 21 shows a schematic of a typical headspace SPME extraction setup for standard, drug or explosive samples. A Varian CP-3800 gas chromatography/Saturn 2000 ion trap mass spectrometry (GC/MS) was used to confirm the presence and quantity of the pre-concentrated analytes on the PDMS fiber. The PDMS fibers were purchased from Supelco, Bellefonte, PA, and the Tuff Syringe field portable SPME fiber holders with Teflon seals were purchased from Field Forensics, St. Petersburg, FL. 

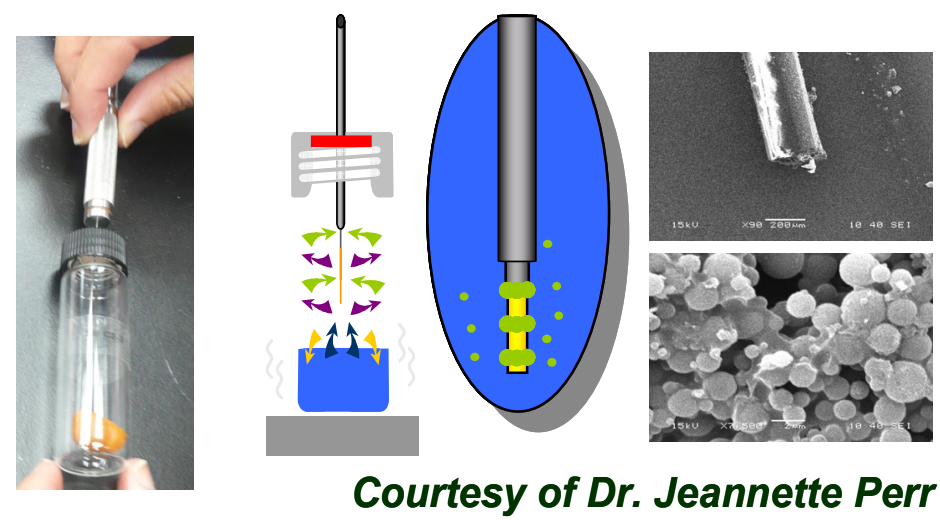

Figure 21. Schematic of headspace SPME extraction setup.

\subsubsection{Calibration Curves for Volatile Components by GC/MS}

The PDMS SPME fiber was exposed to the headspace inside a glass vial containing the sample for a known amount of time. The amount extracted material on the fiber was then analyzed using GC/MS calibration curves to determine the mass extracted. The same extraction procedure was repeated and the fiber was desorbed into the sample introduction area of an IMS to generate a response curve indicating mass vs. intensity.

\subsubsection{SPME Headspace Extraction Limit of Detection by SPME-IMS}

The mass of analytes extracted from the drugs' headspace in the quart cans and the glass vial onto the SPME fiber for each extraction time was calculated from the intensity responses obtained from the IMS analysis using calibration curves. The SPME-IMS calibration curves for each of the volatile components of the drugs in this study were previously determined from standards and are as follows: $y=227.55 x+307.17$ for methyl benzoate (cocaine); $y=372.56 x$ +452.61 piperonal (MDMA); and $y=495.94 \mathrm{x}+1137.2$ for $\alpha / \beta$-pinene and limonene (marijuana). The unit for ' $x$ ' is in nanograms (ng), and ' $y$ ' is in millivolts (mV). A Varian 
(Walnut Creek, CA) CP-3800 gas chromatography/Saturn 2000 ion trap mass spectrometer (GC/MS) was used to confirm the pre-concentrated analytes on the SPME fiber were the volatile constituents of the drugs and not the drug particles or vapors themselves.

\subsection{IMS Reduced Mobility $\left(\mathrm{K}_{0}\right)$ Determination}

Reduced mobilities of the compounds were determined using Smiths Detection IonScan (Mississauga, ON, Canada) 400B Ion Mobility Spectrometer. The instrument was set at optimal operating conditions for the compound of interest and $K_{0(\text { Calibrant })}$ was re-calculated using Equation 10. Diazepam was used as a reference standard material with $K_{0}$ value of $1.21 \mathrm{~cm}^{2} \mathrm{~V}^{-1} \mathrm{~s}^{-1}$.[17] Once the instrument was calibrated, compounds of interest were analyzed and their reduced mobility values were provided by the software. A screen shot of the $K_{0}$ determination by the Smiths software is shown in Figure 22.

$$
K_{0(\text { Analyte })}=K_{0(\text { Calibrant })} \frac{t_{d(\text { Calibrant })}}{t_{d(\text { Analyte })}} \quad \text { Equation } 10
$$
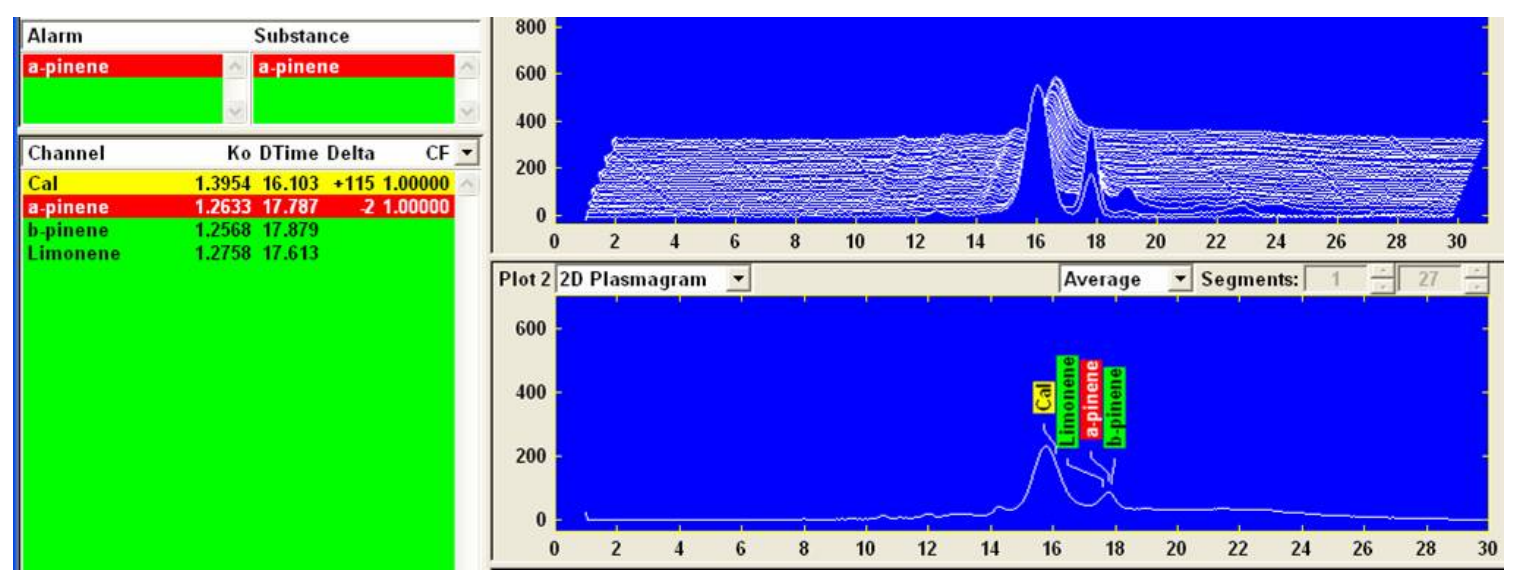

Figure 22. $\mathrm{K}_{0}$ determination from an analysis of the standard using the Smiths IMS. 


\subsection{Mass Quantification for SPME-IMS Response}

\subsubsection{SPME Extraction and Analysis by GC/MS and IMS}

The SPME PDMS fiber was exposed to the headspace inside a glass vial or metal quart can, containing the sample for a known amount of time. The amount extracted material on the fiber was then analyzed using GC/MS calibration curves to determine the mass extracted. The same extraction procedure was repeated and the fiber was desorbed into the sample introduction area of an IMS to generate a response curve indicating mass vs. intensity.

\subsubsection{SPME-IMS LoDs and LDRS}

The limit of detection for each of the compounds was calculated from its response curve. The equation generated from the best fit line of the curve is in the form of $y=m x+b$, where the concentration, " $x$ " can be determined by substituting " $y$ " as the sum of the mean blank signal plus 3 times the standard deviation of the blank. Response curves with data points containing the error bars are based on 3 replicate measurements. The linear dynamic range was determined by identifying the largest range of points on the response curve where there is a linear correlation between mass and response. The student's t-test was used to test for significant correlation on the r-value. Where correlations were found, the best-fit line was plotted and the linear dynamic range was determined.

\subsubsection{Drug Equilibrium Extraction Time Profiles}

All drug samples and household consumable samples were sealed for at least 2 days inside quart sized cans (metal lid with septum hole) to allow the volatile components to reach the 
equilibrium headspace concentration prior to extraction. The can containing 0.5 gram of cocaine $\mathrm{HCl}$ was extracted using a $100 \mu \mathrm{m}$ polydimethyl siloxane (PDMS) SPME fiber (Supelco, Bellefonte, PA) for extraction times of 2, 5, 10, 15, and 30 minutes in triplicates with blank runs in between. A 30 minute waiting period was allowed between each extraction to avoid diminishing the headspace concentrations. The can containing 5 grams of marijuana was extracted for $1,2,3,5$, and 10 minutes. The $50 \mathrm{~mL}$ glass vial containing 0.92 gram of MDMA (4 tablets) was extracted for $10,30,40,60,120$, and 150 minutes in triplicates. The cans were previously baked in the oven, and background spectra of the empty cans were also collected as a blank. All SPME extractions were performed at room temperature and atmospheric pressure. The intensity values corresponding to each extraction time were plotted to form the equilibrium extraction time profile for each of the drugs.

\subsection{Chemicals and Explosive Samples}

Prior to the detection by IMS, standard chemicals were used to calibrate the instrument to determine the expected drift time of corresponding product ion peaks. The following standards were purchased; N-butyl acetate (Acros Organics, New Jersey), cyclohexanone (Fisher Scientific, New Jersey), and DMNB (Aldrich Chemical Company, Wisconsin). Nicotinamide was used as a dopant gas for the analyses in positive operating mode. Empty permeation tubes purchased from VICI Metronics Inc. (Poulsbo, WA) were filled with nicotinamide obtained from Acros Organics (New Jersey). Methanol was used as a solvent for GC/MS liquid calibration. The SPME-GC/MS and SPME-IMS analyses of plastic explosives Composition-4 (C-4), Detasheet, and Semtex H samples were performed at a law enforcement laboratory. 


\subsubsection{Preparation of Explosive Samples}

There were 3 cases of Detasheet explosive provided for this study. Two (2) were Flex X untagged explosives that originated from the same lot but different cuts, and the other was Primasheet 1000 tagged explosive. Three cases of Semtex H were also provided, of which all 3 were tagged; the first two cases were different cuts from the same lot and the third case was from a different lot. $1.0 \mathrm{~g} \pm 0.02$ of these explosives were cut out from the center of the bulk to avoid surface contamination. The $1 \mathrm{~g}$ samples were contained in a $15 \mathrm{~mL}$ glass vial (Supelco, Bellefonte PA) and sealed 1 week prior to analysis. One set of vials was sealed $24 \mathrm{hr}$ prior to analysis. See Table 15 for details on the explosives' origin and years of manufacture.

Table 15. Details of the plastic explosive samples.

\begin{tabular}{|l|l|c|c|}
\hline \multicolumn{1}{|c|}{ EXPLOSIVES } & \multicolumn{1}{|c|}{ Make/Year/Lot \# } & Mass & Seal time \\
\hline Flex X, untagged (Case 1) & $\begin{array}{l}\text { Dupont /1968/ } \\
\text { DU1210268 }\end{array}$ & $1 \mathrm{~g}$ & 7 days \\
\hline Flex X, untagged (Case 2) & $\begin{array}{l}\text { Dupont /1968/ } \\
\text { DU1210268 }\end{array}$ & $1 \mathrm{~g}$ & 7 days \\
\hline PrimaSheet1000, tagged (Case 3) & $\begin{array}{l}\text { Ensign-Brickford/Unknown/ } \\
\text { 04ALL08G1 }\end{array}$ & $1 \mathrm{~g}$ & 7 days \\
\hline Semtex H, tagged (Case 1) & $\begin{array}{l}\text { Explosia Czech } \\
\text { Republic/Unknown/Unknown }\end{array}$ & $1 \mathrm{~g}$ & 7 days \\
\hline Semtex H, tagged (Case 2) & $\begin{array}{l}\text { Explosia Czech } \\
\text { Republic/Unknown/Unknown }\end{array}$ & $1 \mathrm{~g}$ & 7 days \\
\hline Semtex H, tagged (Case 3) & $\begin{array}{l}\text { Explosia Czech } \\
\text { Republic/2002/Unknown }\end{array}$ & $0.8 \mathrm{~g}$ & $7+$ days \\
\hline
\end{tabular}

\subsubsection{SPME Fiber Chemistry Study}

Five different fiber chemistries were used in the study to extract volatiles from the headspace of each of the explosives, Detasheet (untagged, case 2), Semtex H (tagged, case 2), and C-4 (tagged), followed by analysis using GC/MS. The analysis method is presented in Table 16 
Carboxen/Polydimethylsiloxane (CAR/PDMS, StableFlex, $85 \mu \mathrm{m})$, Polydimethylsiloxane (PDMS, $100 \mu \mathrm{m}$ ), Divinylbenzene/Carboxen/ Polydimethylsiloxane (DVB/CAR/PDMS, StableFlex, 50/30 $\mu \mathrm{m})$, Carbowax/ Divinylbenzene (CW/DVB, $70 \mu \mathrm{m})$, and Polydimethylsiloxane/Divinylbenzene (PDMS/DVB, $65 \mu \mathrm{m}$ ) were the chemistry coatings used in the C-4 study, 3 replicates each with a blank run in between. The CW/DVB fiber was replaced with PDMS/DVB (StableFlex $65 \mu \mathrm{m}$ ) in the Detasheet and Semtex H studies because the CW/DVB coating was very easily damaged. Only 1 run with a blank in between samples was performed on each of these two explosives.

Table 16. Operating conditions of IMS, SPME interface, and GC/MS.

\begin{tabular}{|c|c|}
\hline \multicolumn{2}{|l|}{ (i) GE Itemiser 2 Operating Conditions } \\
\hline Interface Temperature $\left({ }^{\circ} \mathrm{C}\right)$ & 60 \\
\hline Drift Tube Temperature $\left({ }^{\circ} \mathrm{C}\right)$ & 50 \\
\hline Sample Flow $\left(\mathrm{mL} \cdot \mathrm{min}^{-1}\right)$ & 1000 \\
\hline Detector Flow $\left(\mathrm{mL} \cdot \mathrm{min}^{-1}\right)$ & 250 \\
\hline Polarity & + \\
\hline Reagent Gas (dopant) & Nicotinamide \\
\hline \multicolumn{2}{|c|}{ (ii) SPME-IMS Interface Operating Conditions } \\
\hline Warm up Time (hr) & 1 \\
\hline Interface Temperature $\left({ }^{\circ} \mathrm{C}\right)$ & $200 \pm 1$ \\
\hline \multicolumn{2}{|l|}{ (iii) GC/MS Operating Conditions } \\
\hline $\begin{array}{l}\text { Agilent } 6890 \mathrm{GC}, 30 \mathrm{~m}, 0.32 \mathrm{~mm} \text { ID, } 1.5 \\
\mu \mathrm{m} \text { df RTX-200 column }\end{array}$ & $\begin{array}{l}50{ }^{\circ} \mathrm{C} \text {, hold } 1 \mathrm{~min} \text {, ramp rate } 10 \\
{ }^{\circ} \mathrm{C} \cdot \mathrm{min}^{-1} \text { to final temp of } 2400^{\circ} \mathrm{C} \text {, } \\
\text { hold } 2 \mathrm{~min} . \text { He } 1.5 \mathrm{~mL} \cdot \mathrm{min}^{-1} \text {. } \\
\text { Injector temp. } 180{ }^{\circ} \mathrm{C} \text {, transfer } \\
\text { line } 180^{\circ} \mathrm{C} \text {. SPME splitless. }\end{array}$ \\
\hline $\begin{array}{l}\text { Agilent } 5973 \text { MSD, with software MSD } \\
\text { ChemStation D01.00 Build } 75\end{array}$ & $\begin{array}{l}\text { Source temp. } 230{ }^{\circ} \mathrm{C} \text {, Quad temp. } \\
150{ }^{\circ} \mathrm{C} \text { EI } 70 \mathrm{eV} \text {, mass range } 40- \\
400 \mathrm{amu} \text {, scan rate } 0.25 \mathrm{~s} / \mathrm{scan}\end{array}$ \\
\hline
\end{tabular}

\subsubsection{SPME extraction quantification and confirmation}

The mass of analytes extracted on the SPME fiber from the headspace of the glass vial containing the explosives for each extraction time was calculated from the intensity responses 
obtained from the IMS analysis using response curves of the standards. The SPME-IMS response curves for the volatile compounds of interest in this study were determined using standards. The response equations are as follows: $y=14 x+1064, R^{2}=0.945$ for $n$-butyl acetate; $y=9 x+344$, $\mathrm{R}^{2}=0.983$ for DMNB, and $\mathrm{y}=55 \mathrm{x}+945, \mathrm{R}^{2}=0.919$ for cyclohexanone. The unit for ' $\mathrm{x}$ ' is in nanograms (ng), and ' $y$ ' is in millivolts $(\mathrm{mV})$. In order to confirm the pre-concentrated analytes on the SPME fiber were the volatile constituents of the explosives and not the explosive particles GC/MS analysis was performed.

\subsubsection{SPME extraction equilibrium}

Vials containing Detasheet Flex X explosive from Case 2 (untagged) were used in the SPME-IMS extraction time experiment for sampling times of $5 \mathrm{sec}, 10 \mathrm{sec}, 20 \mathrm{sec}, 1 \mathrm{~min}, 5 \mathrm{~min}$, $20 \mathrm{~min}$, and $30 \mathrm{~min}$. A minimum waiting period of $30 \mathrm{~min}$ was allowed between each extraction to avoid diminishing the headspace concentrations of the vials in use. The vials containing Semtex H explosive from Case 2 were used for sampling times of $2 \mathrm{~min}, 5,1015,23,60$, and $120 \mathrm{~min}$. For C-4 explosive, sampling times were $10 \mathrm{sec}, 30 \mathrm{sec}, 1 \mathrm{~min}, 2,3,4$, 5, and $10 \mathrm{~min}$. The sampling time was measured from the start of the extraction, when SPME needle punctured the septum of the glass vial and the fiber was exposed, up until the fiber was withdrawn. The mass of analytes recovered corresponding to each extraction time were plotted to form the equilibrium extraction time profile for each of the explosives. All SPME extractions were performed at room temperature, using PDMS/DVB fiber for C-4 and Detasheet Flex X explosives, and PDMS/DVB StableFlex fiber for Semtex H. 


\section{CHAPTER 10: METHOD DEVELOPMENT FOR IMS MODELING AND SIMULATIONS}

CONTENT

10.1 Materials and Instrumentation 106

10.2 Simulation Methodology. 108

10.3 Evaluation of Simulation Method: Empirical vs. Simulation Experiments 114

\subsection{Materials and Instrumentation}

\subsubsection{Chemical and Instrumental Conditions: Empirical vs. Simulation}

The test compounds used in this study were standard explosives and drugs, selected to serve the purpose of collecting data for both positive and negative modes. Explosive standard 2,4,6-trinitrotoluene (TNT) was obtained from Accu Standard Inc. (New Haven, Connecticut). Cocaine standard was obtained from Cerilliant (Round Wrap, TX); 2,7-dinitrofluorene (DNF) was obtained from Aldrich Chem. Co. (Mil, WI). Dynacal permeation devices containing ammonia, and methylene chloride used as dopants were obtained from VICI Metronics Inc.

(Poulsbo, WA) with a permeation rate of $3,100 \mathrm{ng} \cdot \mathrm{min}^{-1} \pm 10 \%$ at $30{ }^{\circ} \mathrm{C}$ and $460 \mathrm{ng} \cdot \mathrm{min}^{-1} \pm 15 \%$ at $30{ }^{\circ} \mathrm{C}$, respectively. The solvents used to prepare test solutions were HPLC grade acetone from Fisher Scientific (New Jersey, NJ) and biotech grade acetonitrile from Sigma Aldrich (St. Luis, $\mathrm{MO})$.

The PCP Phemto-Chem Ion Mobility Spectrometer Model 110 (West Palm Beach, FL) shown in Figure 23 was used in this study to obtain empirical (experimental) data for the compounds of interest. The PCP IMS can operate in both positive and negative mode by varying 
instrumental conditions. The standard or default operating conditions of the PCP IMS 110 were an operating drift tube temperature set at $200{ }^{\circ} \mathrm{C}$, an inlet temperature at $250^{\circ} \mathrm{C}$, and gas-flow rates of 150 and $500 \mathrm{ml} \cdot \mathrm{min}-1 \mathrm{f}$ or carrier gas and drift gas, respectively. All experiments were conducted at the Idaho National Laboratory (INL) in Idaho Falls, ID (elevation $4700 \mathrm{ft}$ ) at atmospheric pressure, which varied from 635 to $650 \mathrm{mmHg}$ for the PCP IMS 110.[131]

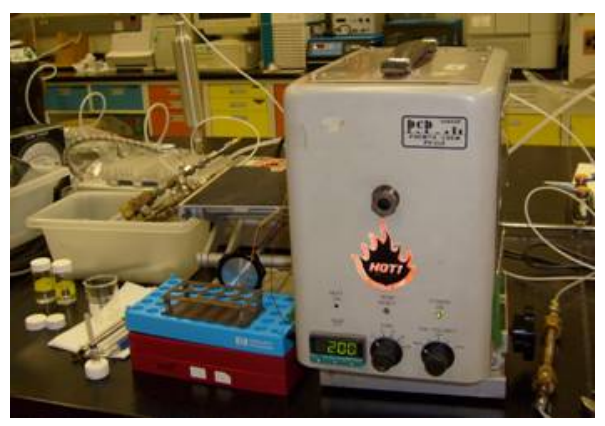

Figure 23. PCP-IMS at Idaho National Laboratory.

Typically, air was used for both carrier and drift gas flows with methylene chloride added to the carrier gas in negative mode, while ammonia dopant was added to the carrier gas in positive mode. The gate width was set at $250 \mu \mathrm{sec}$ with a cycling rate of 40 cycles per second. Standard parameters were used except as noted in Table 17 for Tests 1-11 for both experiments and simulations (unless stated otherwise) to study; 1) drift gas flow rate, 2) drift gas composition, and 3) gate width. The first study of the drift gas flow rate used the test compounds TNT and cocaine. The second study of the drift gas composition used TNT in the single component test, followed by a mixture test using both TNT and DNF. The third study of the ion gate width used TNT as the test compound. For Tests 1 to 11 , experiments were run in triplicate. 
Table 17. IMS conditions for gas flow rate, drift gas composition, and gate width study.

\begin{tabular}{|lcccc|}
\hline $\begin{array}{c}\text { Drift Gas Flow Rate } \\
\left(\mathbf{m l} \cdot \mathbf{m i n}^{-1}\right)\end{array}$ & $\begin{array}{c}\text { Carrier Gas* Flow } \\
\text { Rate }\left(\mathbf{m l} \cdot \mathbf{m i n}^{-1}\right)\end{array}$ & $\begin{array}{c}\text { Drift Gas } \\
\text { Composition }\end{array}$ & $\begin{array}{c}\text { Gate width } \\
(\boldsymbol{\mu s e c})\end{array}$ \\
\hline Gas Flow Rate Study & 500 & 150 & Air & 250 \\
\hline Test 1 & 100 & 100 & Air & 250 \\
\hline Test 2 & 0 & 150 & Air & 250 \\
\hline Test 3 & 500 & 56 & Air & 250 \\
\hline Test 4 & & & \\
\hline Drift Gas Composition Study & 500 & 150 & Air & 250 \\
\hline Test 5 & 500 & 150 & Helium & 250 \\
\hline Test 6 & 500 & 150 & Argon & 250 \\
\hline Test 7 & & & Air & 50 \\
\hline Gate Width Study & 150 & Air & 100 \\
\hline Test 8 & 500 & 150 & Air & 250 \\
\hline Test 9 & 500 & 150 & Air & 500 \\
\hline Test 10 & 500 & 150 & & \\
\hline Test 11 & 500 & & & \\
\hline
\end{tabular}

*Air was used as the carrier gas for all experiments.

\subsubsection{Sample Introduction: Empirical vs. Simulation}

Explosive standard 2,4,6-trinitrotoluene was diluted in acetone solvent to 5ppm. Cocaine and 2,7-dinitrofluorene standards were diluted in acetonitrile to $50 \mathrm{ppm}$. Each sample was introduced by direct liquid injection at the inlet by depositing a $1 \mu \mathrm{L}$ aliquot of the sample solution into the quartz sample tube inside the Teflon ${ }^{\circledR}$ cap, which was then inserted into the hot quartz showerhead tube of the PCP IMS 110. The sample solution in the tube was heated, desorbed, and carried into the IMS reaction region. For simulation, sample introduction was simply the birth of different species of ion without the complication of reactant ion.

\subsection{Simulation Methodology}

\subsubsection{Simulation Strategy and Approach}

The strategy for simulating ion trajectories within the PCP IMS 110 instrument utilized SIMION 7.0 (SIS, Ringoes, NJ) to model the electrostatic fields with the SDS user program [119] 
to account for the viscous pressure affects. The SDS user program can account for gas flow if the $\mathrm{x}, \mathrm{y}$, and $\mathrm{z}$ velocity vectors are provided in separate '.dat' files. Therefore the fluid dynamics program COSMOSFloWorks $^{\circledR}$, in association with SolidWorks 2007 (Concord, MA), was used to determine the gas flow velocity vectors for importing into SDS. Because of the need to superimpose the carrier and/or drift gas flow vectors generated by COSMOSFloWorks onto the grid used by SIMION, the dimensions of the SolidWorks model had to be identical to those of the model in SIMION. In addition, care was taken to locate the origin at a common position in both models to avoid having to make corrections to register the coordinates between the SIMION and SolidWorks models.

The SIMION and SolidWorks computer models of the PCP IMS instrument shown in Figure 24 were constructed to the best possible exactness based on the information available in the manufacturer's manual. Estimations made during the construction of the PCP IMS computer model include some minor details in the drift tube gate dimensions, not shown on the 2D schematic Figure 25. 


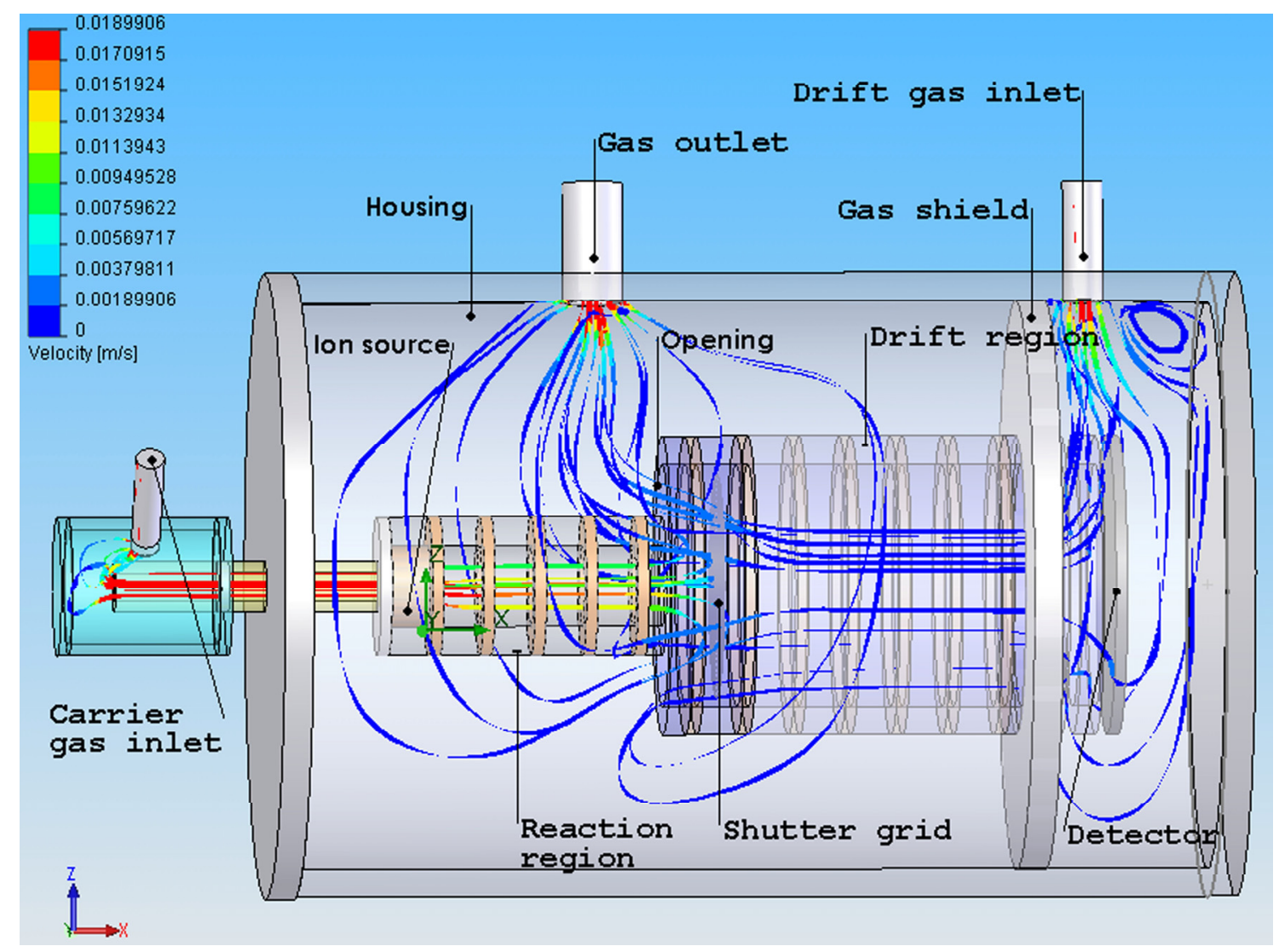

Figure 24. 3D computer model of PCP IMS showing the gas trajectories from the Fluid Dynamic Simulation.

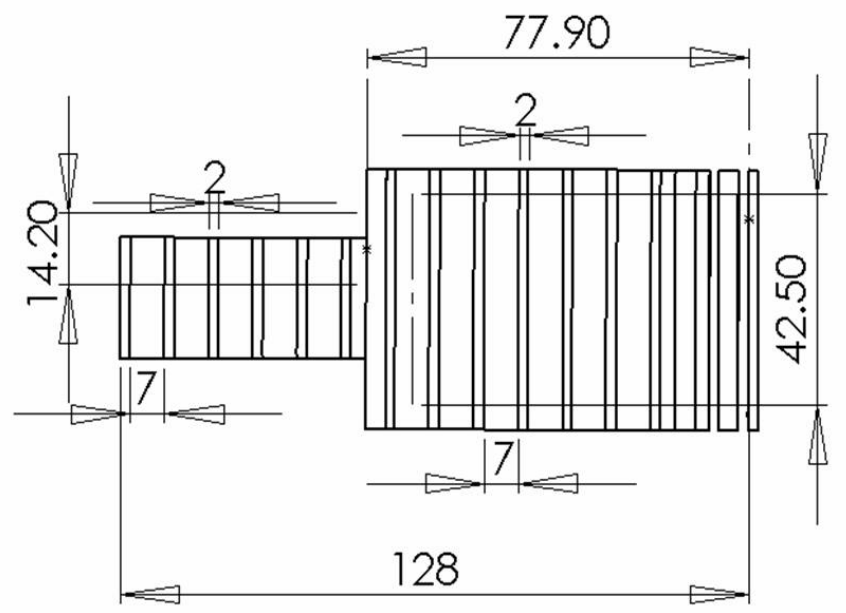

Figure 25. PCP IMS's dimensions (in mm) on 2D drawing extracted from SodidWorks. 


\subsubsection{Assumptions of Computer Model}

Assumptions made in the computer model include the estimates for the geometry of the inlet area, the surrounding gas chamber, and the gate. Of these, the gate geometry and the estimated voltage applied on the gate wires are the features that would have the greatest impact on the accuracy of the simulations. In the real PCP IMS instrument, the gate used to release ions from the ionization region into the drift tube consists of coplanar alternating parallel wires, essentially a Bradbury-Nielson shutter-grid gate.[132] Bradbury-Nielson gates have been described as difficult to fabricate.[17] The difficulty associated with modeling this gate type is that it would require $400 \mathrm{M}$ grid units within SIMION to form a complete 3D model of the instrument because the symmetry of the gate (planar) is not the same as the cylindrical symmetry of the ionization and drift tubes of the PCP IMS instrument and the thin wires require 2 to 4 grid units to model so that they appear as real wires and not an ideal grid.[121] The symmetry options available in SIMION were exploited in order to reduce the computation time by decreasing the number of grid points by modifying the gate geometry. Instead of using alternating gate wires placed next to one another in a planar, rod-like configuration, the gate was created as a circular configuration of alternating wires, which is even more difficult to construct in reality compared to a coplanar Bradbury-Nielson gate. Simple SIMION tests of the two gate configurations revealed that the blocking and releasing of ions should be very similar. This variation of the gate geometry simplified the SIMION model of the complete PCP IMS by allowing the use of symmetry about the central axis and made the computational time manageable at $\sim 2$ hours per simulation using a 3.06 GHz personal computer with $640 \mathrm{MB}$ of RAM.

\subsubsection{Fluid Dynamic Simulations}

SolidWorks and the fluid dynamics COSMOSFloWorks software were used to obtain the flow velocities required by SIMION/SDS to simulate the flow of carrier and drift gases for the 
PCP simulations. Within COSMOSFloWorks, the gas composition and flow rates for the carrier and drift gases at their respective inlets were set according to the conditions for each simulation as given in Table 17. The two gases exit at the same outlet, which was set at atmospheric pressure. After completion of each simulation, the $\mathrm{x}, \mathrm{y}$, and $\mathrm{z}$ flow vectors that corresponded to the SIMION grid positions were extracted from the COSMOSFloWorks results and transposed into individual $\mathrm{x}, \mathrm{y}$, and $\mathrm{z}$ velocity '.dat' files for use by SDS.

\subsubsection{Ion Trajectory Simulations}

SIMION 7.0 software was used to model the PCP IMS ionization reaction region, the drift tube containing the alternating solid electrodes and insulator rings, the gate, and the detector (Figure 26(A)). Figure 26(B) shows an isometric view of the potential energy field for an overall electrical field gradient of $200 \mathrm{~V} \cdot \mathrm{cm}^{-1}$ within the PCP IMS. Ion properties (e.g., charge, mass, and initial kinetic energy) and the total number of ions were defined within SIMION. The $\mathrm{K}_{0}$ values for specific masses were incorporated in the M_DEFS.dat file associated with SDS. While SDS will calculate $\mathrm{K}_{0}$ values for a mass if not provided by the user, these estimated $\mathrm{K}_{0}$ values may be quite different than the actual values because SDS assumes a spherical geometry. The calculated drift times calculated with an SDS estimated $\mathrm{K}_{0}$ can be off by more than $40 \%$ compared to experimental values. 


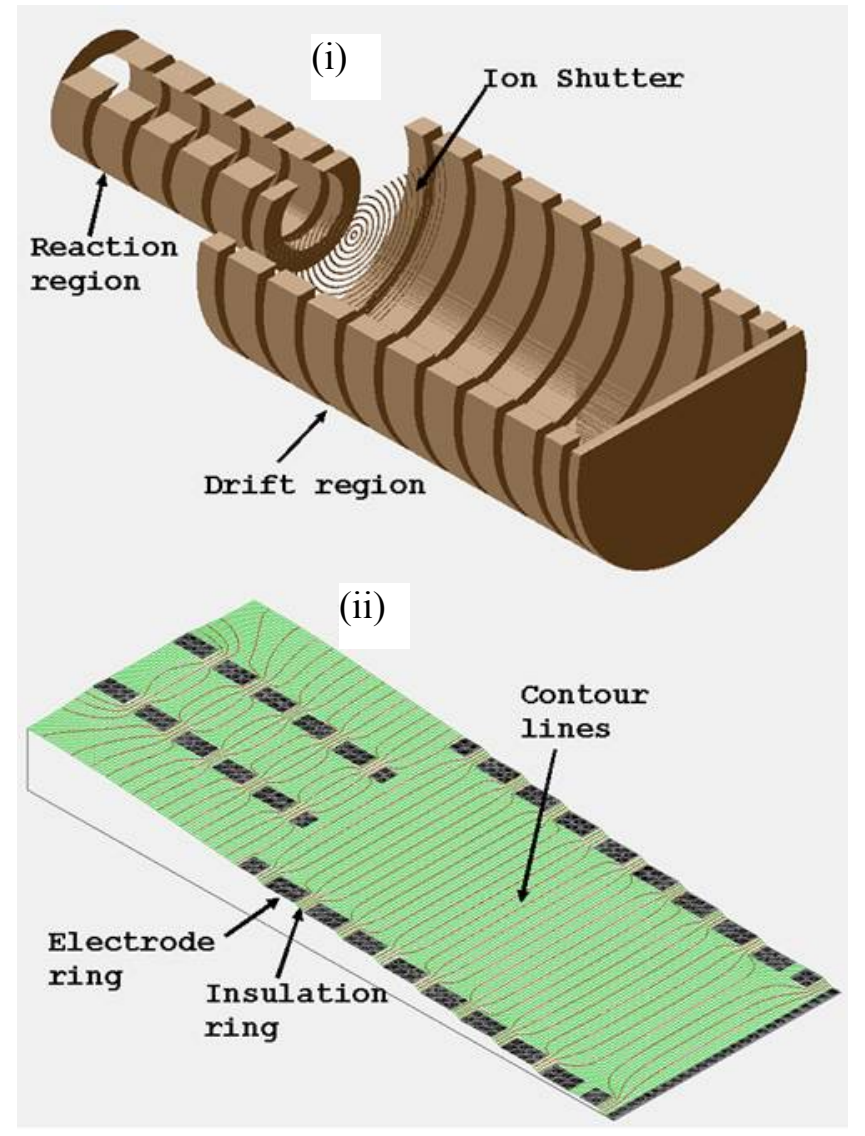

Figure 26. SIMION isometric view (i), and SIMION potential energy field view (ii) of the PCP IMS.

While SIMION allows the initial kinetic energy of the ion to be defined, it should be noted that this is not very important in atmospheric calculations because the ion quickly loses any of its initial kinetic energy to collisions.[121] Space-charge effects, which are available in SIMION, were not included because they are computationally intensive and would be revealed as an increase in effective diffusion.[121] The source code of the SDS user program file was modified to include the change in voltage on the alternating gate wires to control the gate opening/closing mechanism, as well as the birth of ion swarms at random locations within the reaction chamber to simulate the continuous ionization source, ${ }^{63} \mathrm{Ni}$, used in the PCP IMS. The drift tube temperature and pressure, collision gas mass and diameter, and gating parameters were 
all defined in the SDS user program. The ions were then prompted to fly down the model under the influence of the electrical field and the carrier gas flow, pass through the gate, and be subjected to the drift gas. The ions' trajectories and flight times (from birth to the detector) were recorded as output files.

With a $30 \mathrm{msec}$ cycle for a typical IMS, the gate opened for $250 \mu \mathrm{sec}$ and closed for the remaining time, allowing only $0.8 \%$ of the total ions generated to enter the drift region. Four thousands ions were introduced in the ionization chamber during each cycle. If $0.8 \%$ of the ions passed through the gate, only 40 ions would reach the detector. This number of ions was deemed insufficient to generate a smooth histogram. To allow more ions to reach the detector, instead of modeling the exact cycle time of the experimental PCP IMS, the gate closing time for the SIMION PCP model was reduced from 30 to $1.0 \mathrm{msec}$, while the gate open time remained the same at $250 \mu \mathrm{sec}$. This allowed approximately 1000 ions to pass through the gate. In general, the rest of the parameters for the simulations were the same as the respective experiments as noted in Table 17 except as noted for the drift gas composition study.

\subsubsection{Plotting of Simulated Peak}

A recorded output file was saved in text format and later translated into a histogram (spectrum) by plotting only the ions that struck the detector electrode. The histogram was plotted with the $\mathrm{x}$-axis representing the drift time $(\mu \mathrm{sec})$ and the y-axis representing the relative intensity (number of ions that hit the detector within each histogram bin) using a bin size of $50 \mu \mathrm{sec}$. A sample histogram is shown in Figure 27. 


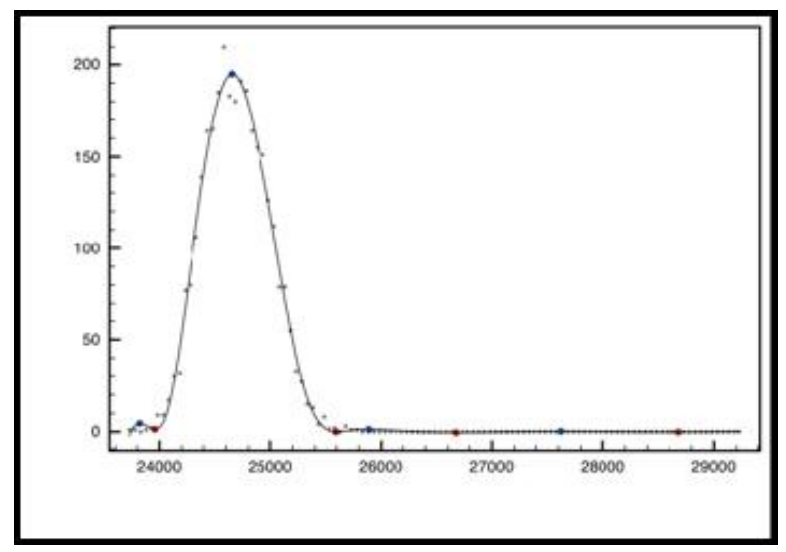

Figure 27. Simulated ion peak from histogram after spline-fitted.

\subsection{Evaluation of Simulation Method: Empirical vs. Simulation Experiments}

\subsubsection{Gas Flow Rates Study}

Simulations 1, 2, 3, and 4 were designed to study the flow rate ratio between the drift and carrier gas at 500:150, 100:100, 0:150, and 500:56 $\mathrm{ml} \cdot \mathrm{min}^{-1}$, respectively. In the various tests of the gas flow rate study, all parameters in SIMION/SDS were kept the same. Only the gas flow vector '.dat' files from COSMOSFloWorks were replaced in SDS for the respective flow rate ratios. The test compound used in the negative mode was TNT, while cocaine was used for the positive mode.

\subsubsection{Drift Gas Composition Study}

In Simulations 5, 6, and 7, the drift gases used were air, helium, and argon, respectively. In the actual experiments, the carrier gas was air. While COSMOSFloWorks can accommodate multiple gases introduced at various inlets, SDS cannot. In SDS, only one type of collision gas can be defined; therefore, this was set as the drift gas. Because a change in the drift gas also changed the carrier gas, the gate timing had to be adjusted for each gas. In air, the time taken for 
the ion swarms to reach the gate was approximately $8700 \mu \mathrm{sec}$, thus the gate was prompted to open with this timing. For helium and argon the gate timing was changed to 2700 and $7000 \mu \mathrm{sec}$, respectively. Two groups of ion species were created, (TNT-H) ${ }^{-}$and (DNF-H)', with 400 ions and 4000 ions, respectively, to mimic the 5:50 ppm aliquot used in the experimental analysis. The two ion swarms were spawned simultaneously. As expected, the two ion swarms separated as they migrated down the drift tube and the resolution between these two adjacent peaks was calculated for each drift gas type using Equation 11, where $\left(t_{d}\right)$ is the ion drift time and $\left(w_{b}\right)$ is the peak full width at base (in $\mu \mathrm{sec})$. $[105,133,134]$ The peak full width at base and full width at half maximum were measured based on standard analytical method, which can be found in the literature.

$$
R_{p p}=\frac{2\left(t_{d 2}-t_{d 1}\right)}{w_{b 1}+w_{b 2}}
$$

\subsubsection{Gate Width Study}

Simulations $8,9,10$, and 11 were set at gate widths of $50,100,250$, and $500 \mu \mathrm{sec}$ respectively. All SDS parameters except for the gate width were kept at the default conditions. The simulations were performed in negative mode with TNT as test compound to mimic the experiments. 
CHAPTER 11: CONSTRUCTION OF AN IN-HOUSE DESIGNED IMS

CONTENT

PAGE

11.1 IMS Physical Components....................................... 118

11.2 Electrical connections..........................................125

11.3 IMS Hardware Components....................................127

11.4 IMS Software Components......................................132

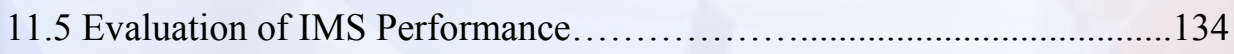
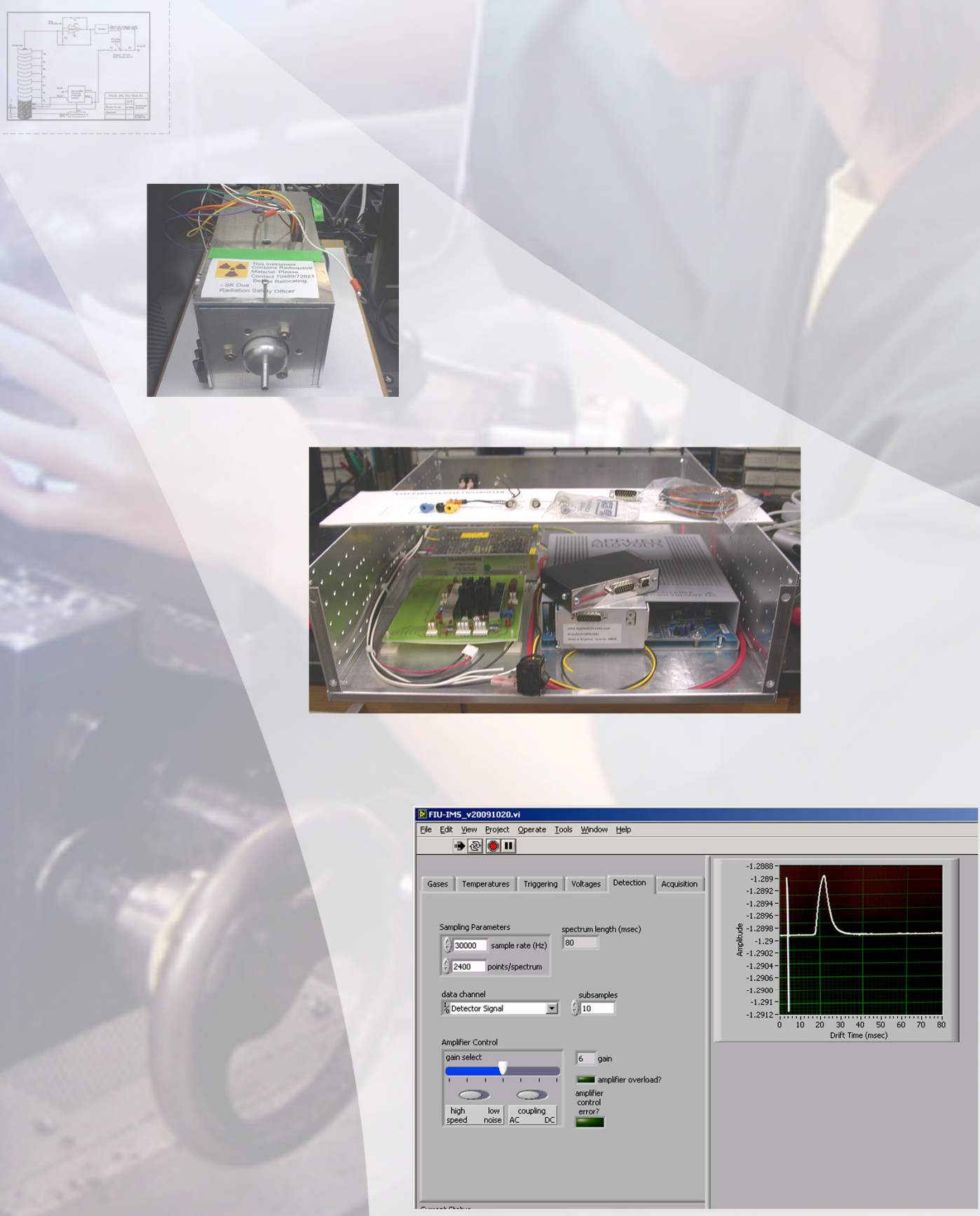


\subsection{IMS Physical Components}

The main components that make up the IMS drift tube consist of 1) the sample inlet, 2) the ${ }^{63} \mathrm{Ni}$ ionization source, 3) conductive rings and insulation rings, 4) the Bradbury-Neilson gate and aperture grid, 5) Faraday detector and shield cup, 6) the HV divider, 7) drift tube metal fixture and enclosure, and 8) Ferrite drift tube design. These components are annotated and shown in Figure 28.

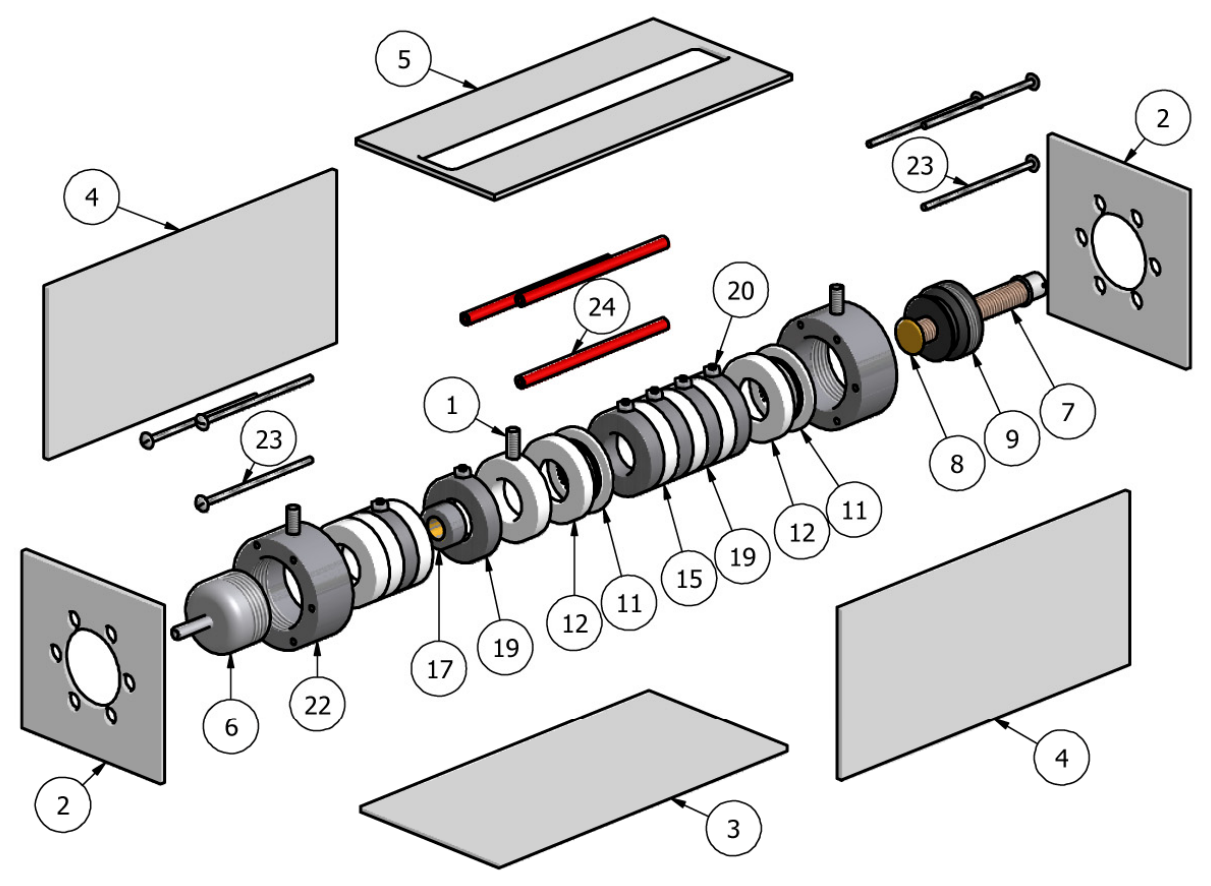

\begin{tabular}{|c|c|l|}
\hline \multicolumn{3}{|c|}{ PARTS LIST } \\
\hline ITEM & QTY & \multicolumn{1}{|c|}{ PART NUMBER } \\
\hline 1 & 3 & Gas Line Connection Tube \\
\hline 2 & 2 & Box End \\
\hline 3 & 1 & Box Floor \\
\hline 4 & 2 & Box Side \\
\hline 5 & 1 & Box Top \\
\hline 6 & 1 & Inlet Nozzle \\
\hline 7 & 1 & Detector Rod \\
\hline 8 & 1 & Detector Plate \\
\hline 9 & 1 & Anious \\
\hline 10 & 1 & BNC Connector \\
\hline 11 & 2 & Gate Ring (Male) \\
\hline 12 & 2 & Gate Ring (Female) \\
\hline
\end{tabular}

\begin{tabular}{|c|c|l|}
\hline \multicolumn{3}{|c|}{ PARTS LIST } \\
\hline ITEM & QTY & \multicolumn{1}{|c|}{ PART NUMBER } \\
\hline 13 & 1 & Gate Wires \\
\hline 14 & 1 & Aperture Grid Wires \\
\hline 15 & 6 & Insulating Ring \\
\hline 16 & 1 & Gas Outlet Ring \\
\hline 17 & 1 & Ionization Source Plug \\
\hline 18 & 1 & Ionization Source 63NI \\
\hline 19 & 6 & Conductive Ring \\
\hline 20 & 6 & High Voltage Connector \\
\hline 21 & 6 & High Voltage Connector Nut \\
\hline 22 & 2 & End Cap \\
\hline 23 & 6 & Rod Bolt \\
\hline 24 & 3 & Ceramic Rod \\
\hline
\end{tabular}

Figure 28. 3D Autodesk Inventor exploded-view of the IMS physical components assembly 


\subsubsection{Sample Inlet}

The sample inlet, shown in Figure 29, was manufactured at the FIU Engineering Manufacturing Lab (Miami, FL) using 6061 Aluminum. The nozzle is threaded for simple screw assembly onto the front of drift tube holder. The extended tube length and diameter were sized with a slip on fit for adaptation to the SPME-IMS interface and the GE heated desorber device as shown in Figure 30.
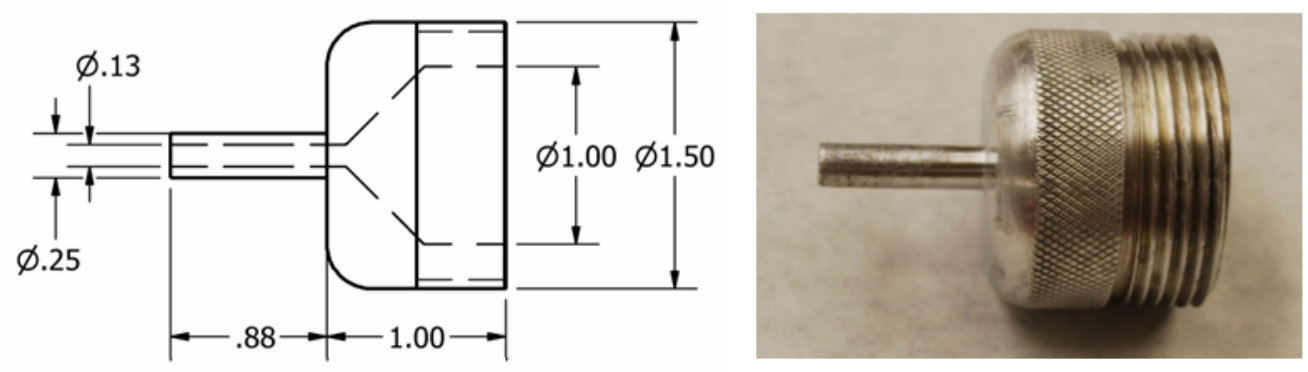

Figure 29. FIU-IMS aluminum inlet.

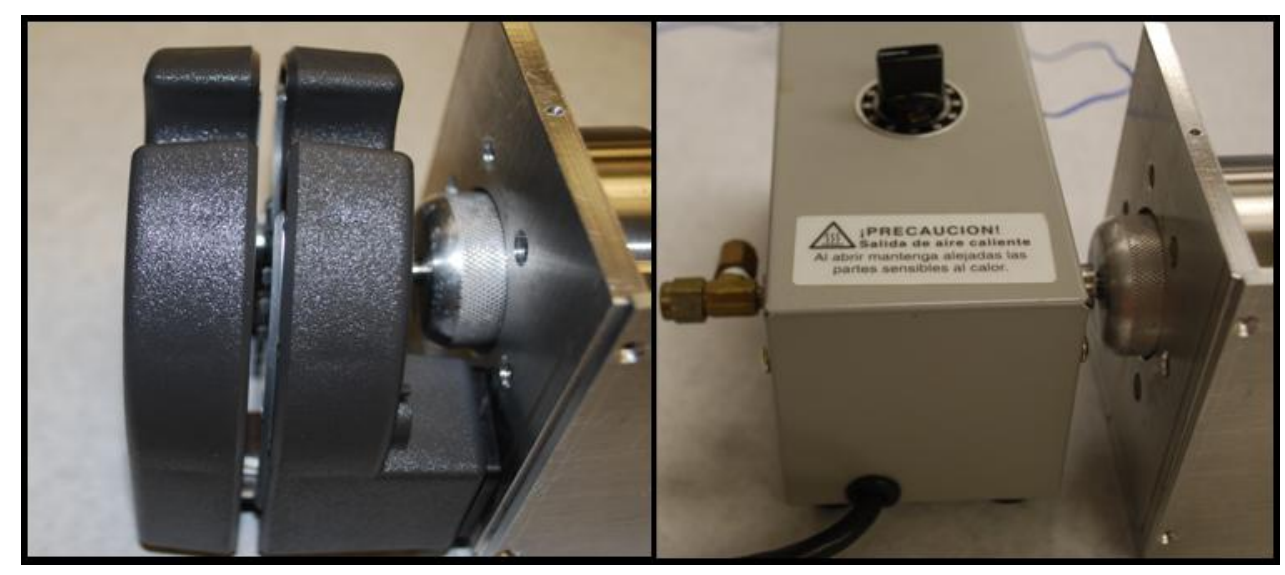

Figure 30. GE desorber and SPME interface with aluminum inlet. 


\subsubsection{Ionization Source}

The ${ }^{63} \mathrm{Ni}$ ionization source, shown in Figure 31 was custom ordered from Eckert \& Ziegler Isotope Products (Valencia, CA). A $15 \mathrm{mCi}$ activity ${ }^{63} \mathrm{Ni}$ radioactive material was plated onto one side of a thin Nickel foil, with the dimensions shown in Figure 31 (left). The foil was inserted into a stainless steel plug (without adhesive material). The plug was machined for a press-fit into one of the concentric conductive rings, insulation rings, or ferrite rings of the drift tube. The use of the plug allowed the source foil to be repositioned in alternative drift tube ring designs without physically handling the foil. The actual source shipped to FIU is shown in Figure 31 (right).
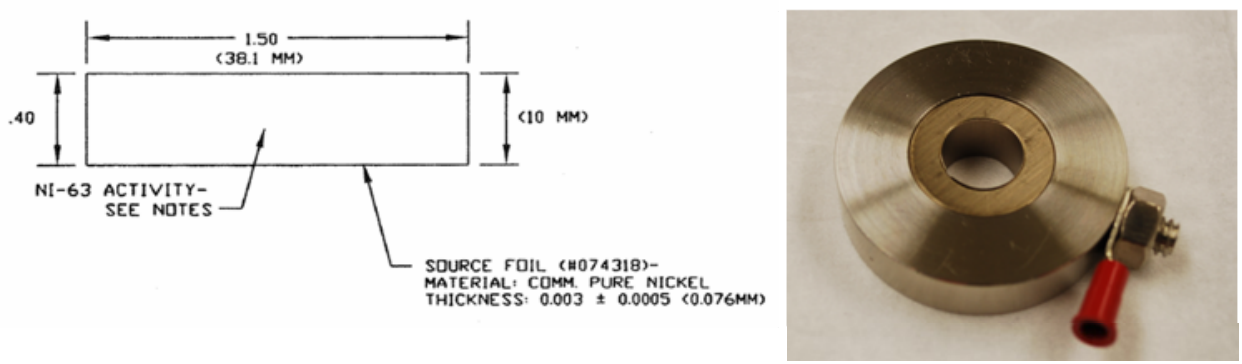

Figure $31 .{ }^{63} \mathrm{Ni}$ ionization source (Nickel foil dimension) inserted inside a conductive ring.

\subsubsection{Conductive and Insulation Rings}

The conductive rings and insulation rings used in the stacked IMS drift tube design are shown in Figure 32. A series of these rings stacked together make up the length of the drift tube. The conductive rings and the insulator rings were machined at the FIU machine shops (Miami, FL) to have the same dimensions of $13 / 4$ " OD, 7/8" ID, and 0.4 " thickness. The conductive rings were machined from 304 stainless steel tube, and the insulation rings were made from Teflon ${ }^{\circledR}$ 
(polytetrafluoroethylene, PTFE) rod. Both materials were purchased from McMaster-Carr (Atlanta, GA).

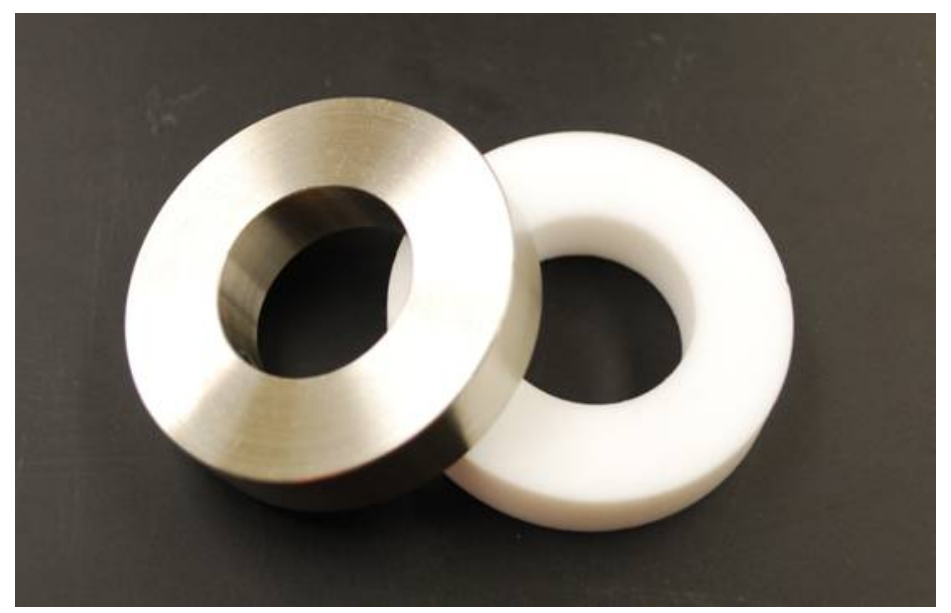

Figure 32. Drift tube conductive and insulation rings.

\subsubsection{Ion Shutter and Aperture Grid}

The Bradbury-Neilson gate (ion shutter), shown in Figure 33, was placed at desirable location along the drift tube, one or two rings after the ${ }^{63} \mathrm{Ni}$ ionization source. The gate was built by wiring two sets of 0.01 " diameter Nickel wire (purchased from McMaster- Carr, Atlanta, GA) through the two rows of holes on a Teflon fixture. The Teflon fixture was machined at the FIU Engineering Manufacturing Lab having the same dimensions as the drift tube rings. The first row contains 10 holes, and the second row contains 10 holes. The holes are 0.025 " in diameter with hole centers 0.075 " apart. The distance between the first row (wire 1) and the second row (wire 2) is 0.058 " or $1.45 \mathrm{~mm}$. The 2 wires were threaded through the holes carefully without kinks, and are free of contact at all points. A separate Teflon piece was machined to cap the Teflon gate fixture so that the gate ring would not allow gas to flow out of the holes through which the wires go. 


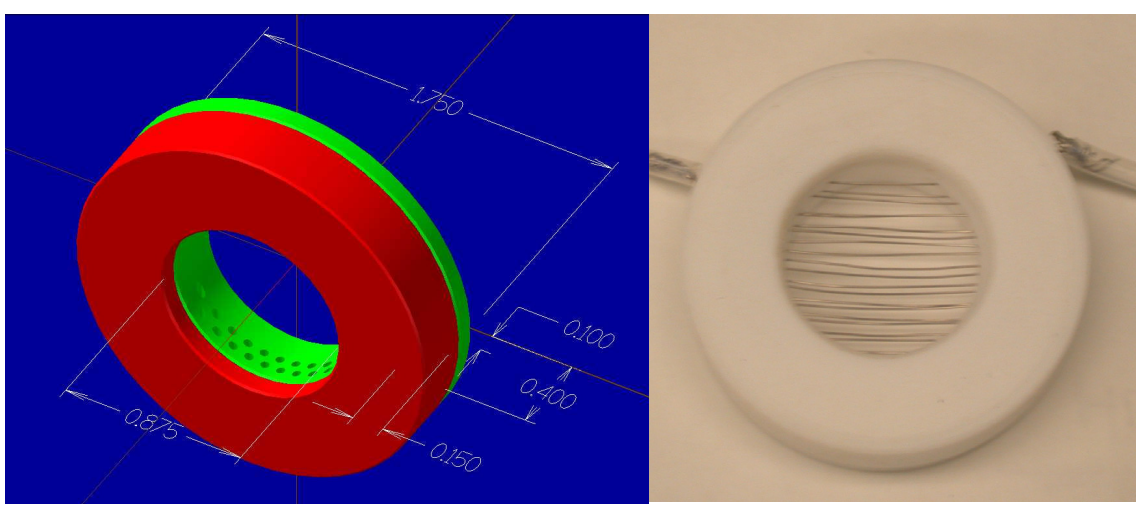

Figure 33. Badbury-Neilson Gate.

The aperture grid ring was machined identically to the $\mathrm{BN}$-gate ring. However, only one set of wires was threaded through one row of holes because the aperture grid sits at only one voltage.

\subsubsection{Faraday Plate and Shield Cup}

The Faraday detector, shown in Figure 34, was made from a simple brass plate with a small hole in the middle. The brass plate was made to connect to the SHV (shielded high voltage) connector using a 20 Gauge enamel coated copper electrical wire (purchased from Small Parts, Miramar FL). The enamel was scraped off on the two ends of the wire for good electrical contact. One end of the wire was soldered (non-leaded) onto the center of the brass plate, and the other end was threaded through a high temperature resistant 3" long threaded rod. The copper wire was cut to the length of the rod and soldered to the connection pin on the SHV connector. The threaded rod was placed through the anious end cap to allow easy adjustment of the detector plate position along the inside the drift tube. The anious (end cap) where the threaded rod screws in place was made of Vespel polyemit, purchased and machined at INL. The outer face of the round anious was threaded for a screw fit onto the back end of the drift tube. The geometry of the anious 
was optimized to improve homogeneous distribution of the inlet drift gas flow coming from the back of the detector. The assembled Faraday detector is shown in Figure 34.

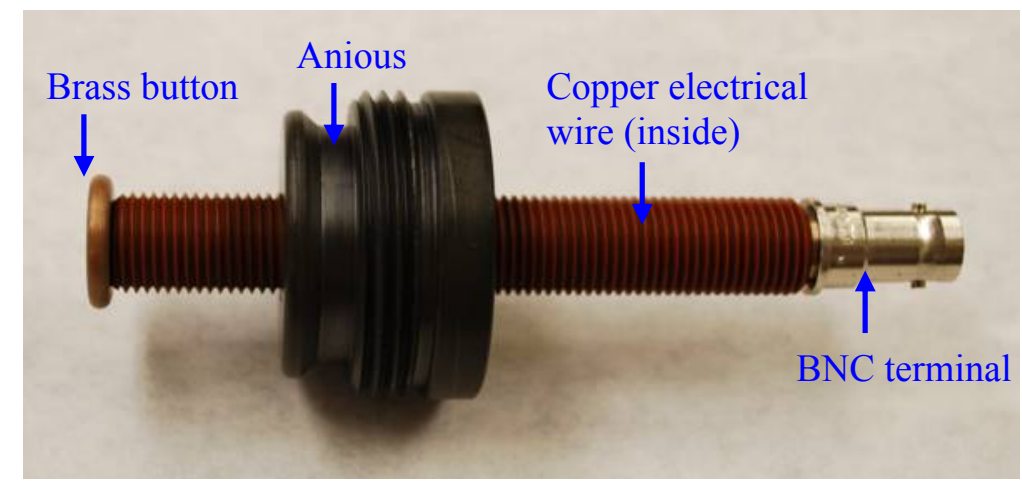

Figure 34. Faraday plate and end cap assembly.

The faraday shield cup, shown in Figure 35, was made from copper metal mesh wire for shielding the Faraday plate and SHV connection from electrical noises in the surrounding and background. This component was found to be essential to improve $\mathrm{S} / \mathrm{N}$ ratio in the mobility spectrum.

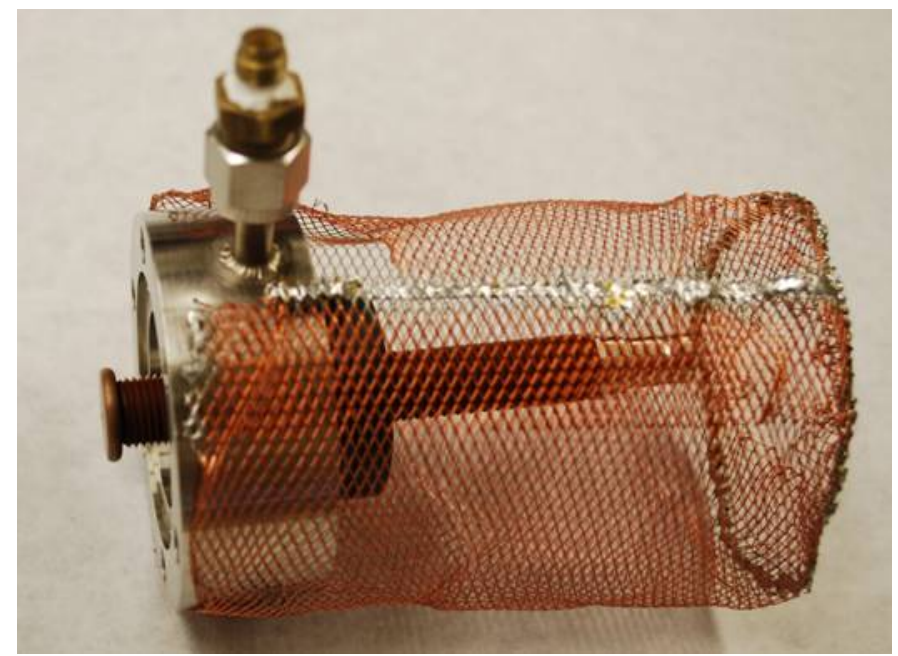

Figure 35. Detector with Faraday shield cup. 
The high voltage divider, shown in Figure 36, was constructed to provide the voltage gradient to the conductive rings. It also provided the voltage for the gate $\mathrm{V}_{\text {ref }}$ and the voltage for the aperture grid. It was made from a series (no. of resistors depending on the no. of conductive rings) of fixed resistors, $\mathrm{R}_{\mathrm{F}}(680 \mathrm{k} \Omega, 1 \%$ tolerance, 2 Watt), and 2 adjustable resistors (500 and $1000 \mathrm{k} \Omega, 2$ Watt) purchased from Digi-Key (Thief River Falls, MN). The reference voltage for the gate $\mathrm{V}_{\text {ref }}$ was taken at point $\mathrm{R}_{\mathrm{A} 1}$, and the voltage for the aperture grid was tapped at $\mathrm{R}_{\mathrm{A} 2}$. The last drift ring was not grounded because that would cause ions to be attracted to the ring instead of the floating Faraday plate. The HV divider was situated outside of the drift tube housing because the resistors are not heat resistant.

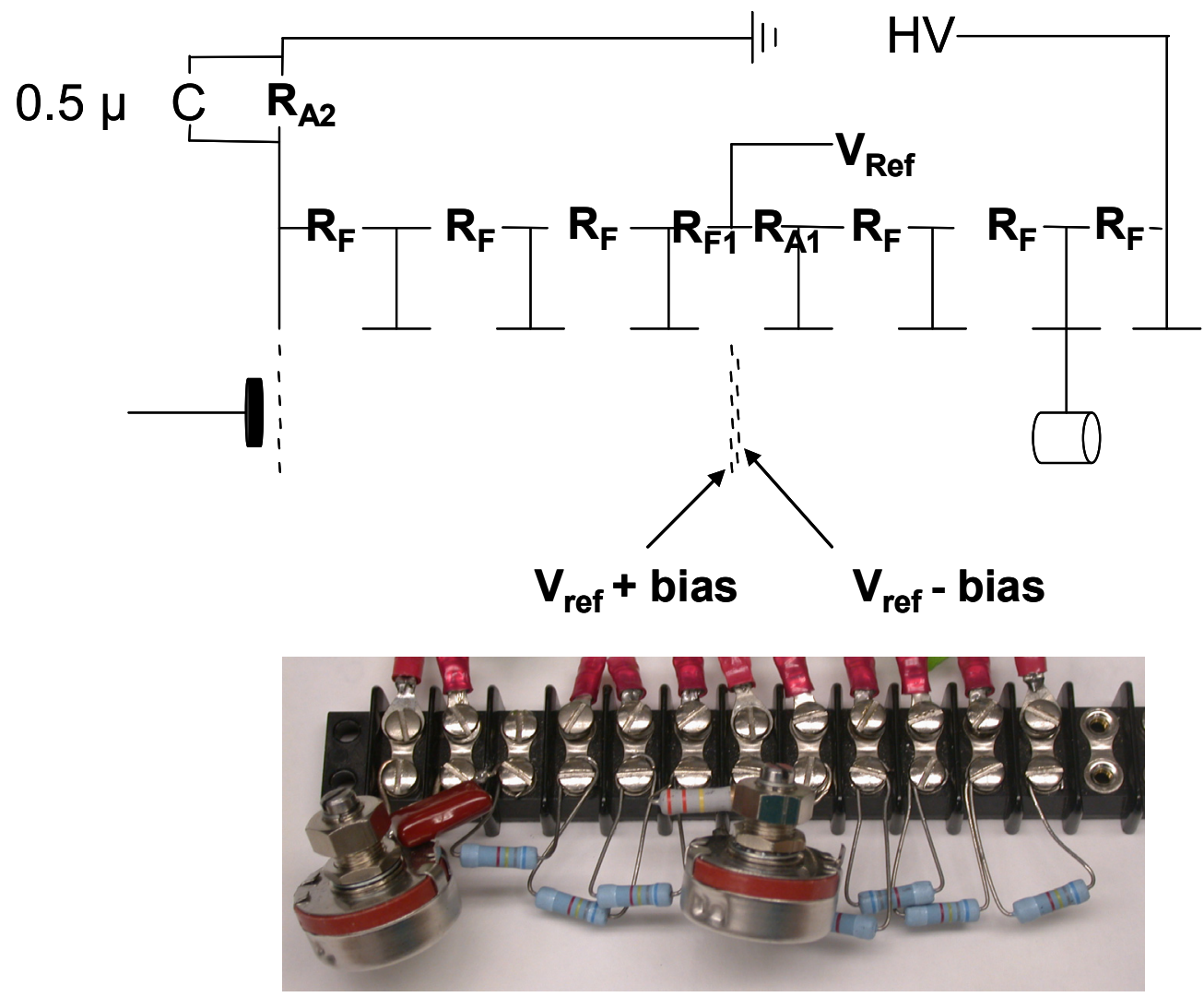

Figure 36. High voltage divider. 


\subsubsection{Assembled Drift Tube and Enclosure}

The drift tube, shown in Figure 37, was assembled by stacking an insulation ring between every pair of conductive rings, with both ends being an insulation ring. The stacked drift tube was held together in a rigid assembly with the help of the two round stainless steel ends and 3 sets of ceramic rods with threaded screw for adjustable tube length (machined at FIU). An Aluminum casing was machined to hold the entire drift tube assembly with extra space for the heating tape and glass wool for insulation.

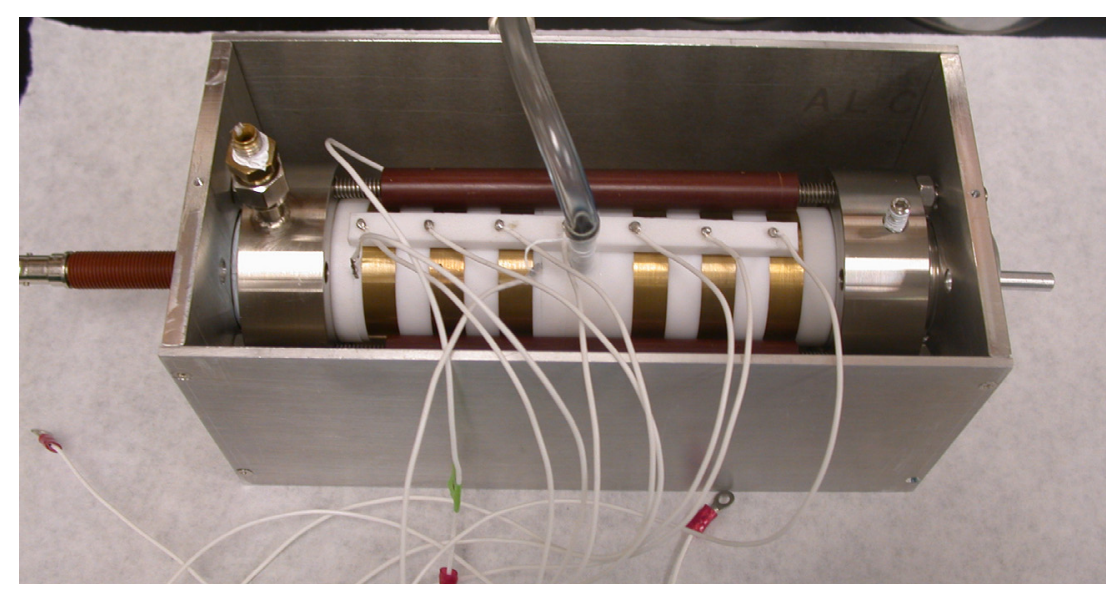

Figure 37. Drift tube in housing after assembly.

\subsection{Electrical connections}

All of the physical components in the IMS that are made of metal, except the Faraday plate, were connected either to earth ground, the HV divider, or the power box outputs. These electrical connections included 1) the HV output to the HV divider which supplies the voltage gradient for the conductive rings, 2) the aperture grid, 3) the BN gate, and 4) drift tube metal fixture and enclosure. 
The HV terminal was connected to the first resistor on the voltage divider and to the first conductive ring of the drift tube (looking from the inlet end). The ground terminal was connected to the last resistor $\left(\mathrm{R}_{\mathrm{A} 2}\right)$ after the aperture grid. Teflon ${ }^{\circledR}$ insulated electrical wire was used to connect each of the conductive rings to their corresponding take-off points on the high voltage divider because some portion of the wire is required to sit at high temperature within the tube housing.

The aperture grid was connected to the HV divider before the last resistor $\left(\mathrm{R}_{\mathrm{A} 2}\right)$. The two terminals on the $\mathrm{BN}$ gate $\mathrm{W}_{1}$ and $\mathrm{W}_{2}$ were connected to the output gate bias voltage connections taken from the main power box.

All the remaining metal pieces such as metal ends, threaded rods, aluminum housing, aluminum inlet, and the Faraday shield cup were made to be connected to reference ground so that they do not distort the field lines within the actual drift tube. To protect the electrical components, prior to supplying high voltage to the tube, several continuity tests between components were made using a standard handheld multi-meter.

Components that were in electrical contact are; 1) each of the conductive rings and its respective position on the HV divider, 2) the aperture grid and its position on the HV divider, 3) the $63 \mathrm{Ni}$ tube and the source conductive ring, and 4) the grounded components.

Components were not in electrical contact which each other are; 1) the aperture grid and the detector plate, 2) the two gate wires, $\mathrm{W}_{1}$ and $\mathrm{W}_{2}, 3$ ) the conductive rings amongst themselves, and 4) the grounded components and the HV components. 


\subsection{IMS Hardware Components}

The hardware components are the accessories that are not part of the IMS physical drift tube but are all essential components in the operation of an IMS detector. These include 1) the current amplifier, 2) the temperature and controller systems, 3) the flow controller systems, 4) the main power supply and the gate switch, and 5) the data acquisition card.

\subsubsection{Current Amplifier}

The current amplifier is used to amplify a very small current signal to a readable DC voltage. The in-house IMS was equipped with a variable gain $\left(10^{3}\right.$ to $\left.10^{11}\right)$ low noise current amplifier model DLPCA-200 (FEMTO, Berlin Germany). The amplifier was integrated into the IMS LabView software program to allow remote control of its settings. The Keithley current amplifier model 428 (Cleveland, OH) was also available for the IMS set up. It operates for gain setting from $10^{3}$ to $10^{11}$ with DC input resistance ranging from $0.6 \Omega$ to $100 \mathrm{k} \Omega$, and within $\mu \mathrm{sec}$ rise time. The actual ion current (in ampere) hitting the detector can was calculated from the output DC voltage observed after amplification at a selected gain value as shown in the sample calculations below. Assuming the observed peak intensity (DC voltage) is $1 \mathrm{~V}$, the gain on the current amplifier was set at $10^{6}$, and the corresponding DC input resistance was $10 \Omega$. Then the ion current was calculated to be $10^{-7}$ Amperes.

$$
I=\frac{V}{R}=\frac{V_{\text {observed }} / \text { Gain }}{\text { Input } \operatorname{Re} \operatorname{sis} \tan c e}=\frac{1 \text { Volt } / 10^{6}}{10 \Omega}=10^{-7} \mathrm{~A}
$$




\subsubsection{Heating System}

The heating and temperature controller system is composed of 5 components purchased from Omega Engineering (Stamford, CT); a I16 controller (part \# CNI16D43-EI), a solid state relay (part \# SSRL240DC10), a finned heat sink (part \# FHS-2), fiberglass heavy heating tape (part \# FGH051-080), and a thermocouple type TJ (part \# KMTSS-062U-6). The in-house IMS was equipped with 2 software controlled versions of this system. One is used for heating and maintaining the temperature of the drift tube, and the other is used for pre-heating the sample and drift gas lines. The thermocouples were inserted directly into the gas phase for accurate measurement of the temperature. The maximum temperature of the heating systems is $\sim 400{ }^{\circ} \mathrm{C}$, which is well above the requirement for the IMS analysis temperature $\left(30^{\circ} \mathrm{C}\right.$ to $\left.200{ }^{\circ} \mathrm{C}\right)$. To protect the heating tapes from high voltage despite wrapping the drift tube, a layer of electrical insulation was placed in between such that the heating tapes do not come into electrical contact with the high voltage rings at any point.

\subsubsection{Gas Flow Controller System}

Two FMA-2605A flow controller systems were purchased from Omega Engineering to control and monitor the sample gas flow and the drift gas flow within the IMS. These systems operate for flow rates up to $2 \mathrm{~L} / \mathrm{min}$, with $\pm 0.8 \%$ reading accuracy, and a $100 \mathrm{msec}$ response time. Both systems were integrated for remote control by the IMS software, but could also be controlled manually. The gas flow system diagram in Figure 38 shows that clean air is directed from a gas cylinder through the drierite container and splits into two gas lines at a t-connector. One gas line is for the sample carrier gas, which passes through flow controller \#1, is preheated, and passes through the dopant container before entering the inlet of the drift tube. The second gas line is for the drift gas, which passes through flow controller \#2 and is preheated prior to entering 
the drift tube from the detector end. The two gases meet at the same outlet situated after the ionization source and are directed through a scrubber unit and then out to the environment. Metal tubing (purchased from Swaglok) was bent into coils and wrapped with heating tape to preheat the drift gas and sample gas, while other connections were made with Teflon tubing.

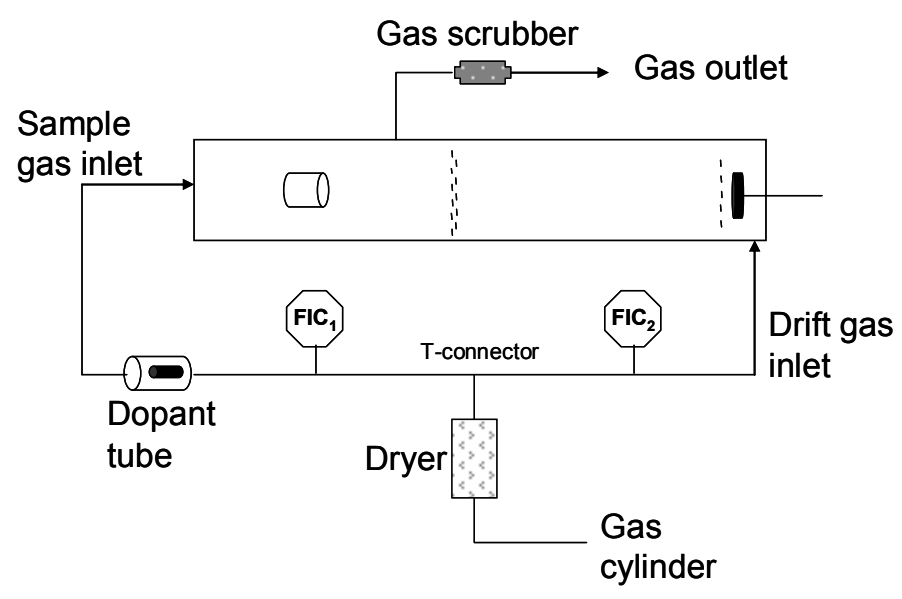

Figure 38. Gas flow diagram of the FIU IMS system.

\subsubsection{Power Supply and Gate Switch}

The IMS power supply box, drawn and pictured in Figure 39, consists of; 1) the high voltage (HV) power supply to provide the electrical field of the drift tube, 2) the bias power supply for the offset voltage on the gate wires, 3) the floating hot deck to float the gate wire voltage at a $\mathrm{HV}$ reference to the drift tube, and 4) the gate switch circuit board. The $20 \mathrm{kV}$ reversible power supply (HP020RAA025) was purchased from Applied Kilovolts (Timonium, MD) and was put together at the FIU physics department electronic shop. Two standard power supplies (24 V and $12 \mathrm{~V} \mathrm{DC)} \mathrm{were} \mathrm{purchased} \mathrm{from} \mathrm{Transitronix} \mathrm{(Ronkonkoma,} \mathrm{NY)} \mathrm{to} \mathrm{supply}$ power to the HV box and the floating hot deck, respectively. The hot deck (12FL-12W-I/O-RB) 
was purchased from Ultravolt. Aside from being a floating ground, it also provides $+12 \mathrm{VDC}+/-$ 5\%@up to 1A, -12VDC +/-10\%@up to 10mA, and +5.6VDC +/-5\% @ up to 10mA. Powering the gate bias uses up a significant portion of the available $+12 \mathrm{~V}$ DC power from the hot deck, and the gate switches were powered from the +5.6 VDC supply. All of the above components were contained inside the metal rack shown in Figure 39.
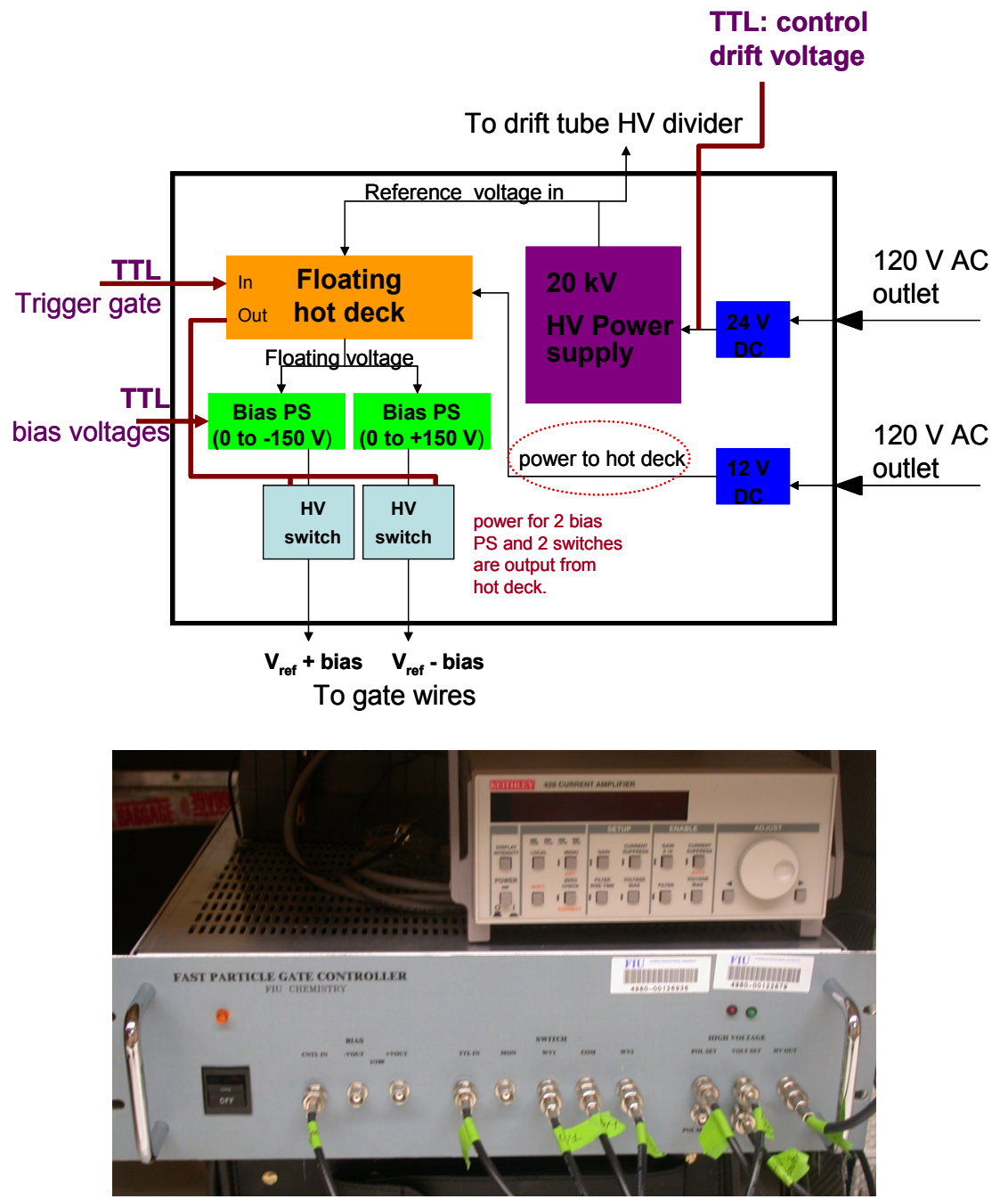

Figure 39. HV drift tube power supply/ion shutter switch box and Keithley amplifier.

The circuit driving the gate switch was floated on the hot deck, where the TTL

(transistor-transistor logic) controls were referenced to ground. On the high voltage side there 
were three inputs (the reference voltage from the HV divider, and the two bias voltages $\pm 150 \mathrm{~V}$ ), and two outputs (the $\mathrm{V}_{\text {ref }}+\mathrm{V}_{\text {bias }}$ and $\mathrm{V}_{\text {ref }}-\mathrm{V}_{\text {bias }}$ ) which were directed to the gate wires as shown in Figure 39 . The gate bias voltages were expected to be operated in the 50 to $150 \mathrm{~V}$ range, thus the gate switch electronics were optimized for operation at $150 \mathrm{~V}$. The gate switch was custom built at the FIU electronic shop, achieved a switching speed of $10 \mu \mathrm{sec}$. The switch needed to be controlled remotely by the IMS software. Its ON/OFF state was driven by the polarity of a TTL signal.

\subsubsection{Data Acquisition Hardware}

The data acquisition hardware consists of 3 components that were purchased from National Instruments (Austin, TX); 1) the PCI-6289 M Series DAQ card (32 Analog Inputs, 2) 48 Digital I/O, 4 Analog Outputs) with NI-DAQmx driver software (installed in the IMS computer), and 3) the BNC-2100 shielded connector blocks (SCC-68 I/O Connector) with signal-labeled BNC connectors. The BNC block is connected to the DAQ card on the IMS computer through a 1 meter long SHC68-68-EPM Shielded Cable (68-Pos. D-Type to 68-Pos. VHDCI offset) shown in Figure 40 .

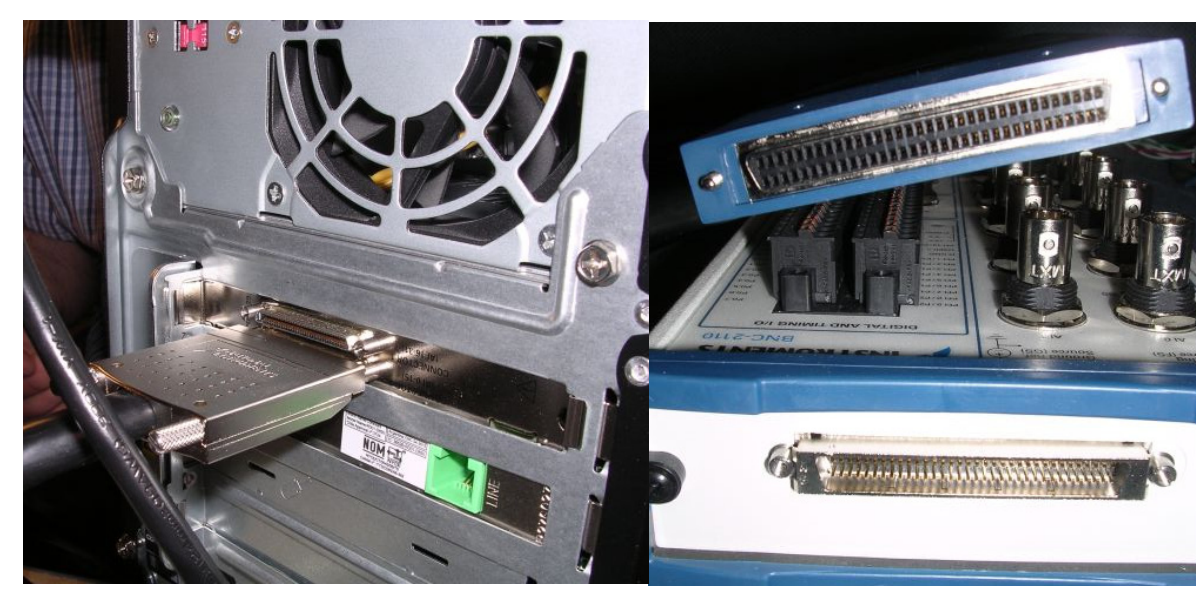

Figure 40. BNC connector box to DAQ card on IMS computer. 


\subsection{IMS Software Components}

The specification of the IMS software was laid out and was provided to Argon Street Laboratories LLC (Columbia, MO), which was contracted to write the code using LabView.

\subsubsection{Hardware Integration and Control of Operating Parameters}

The generalized diagram in Figure 41 shows the information flow in the IMS software, with blocks representing the various subsystems and lines representing the flow of control signals and read-backs, data acquisition, and data file formatting and storage.

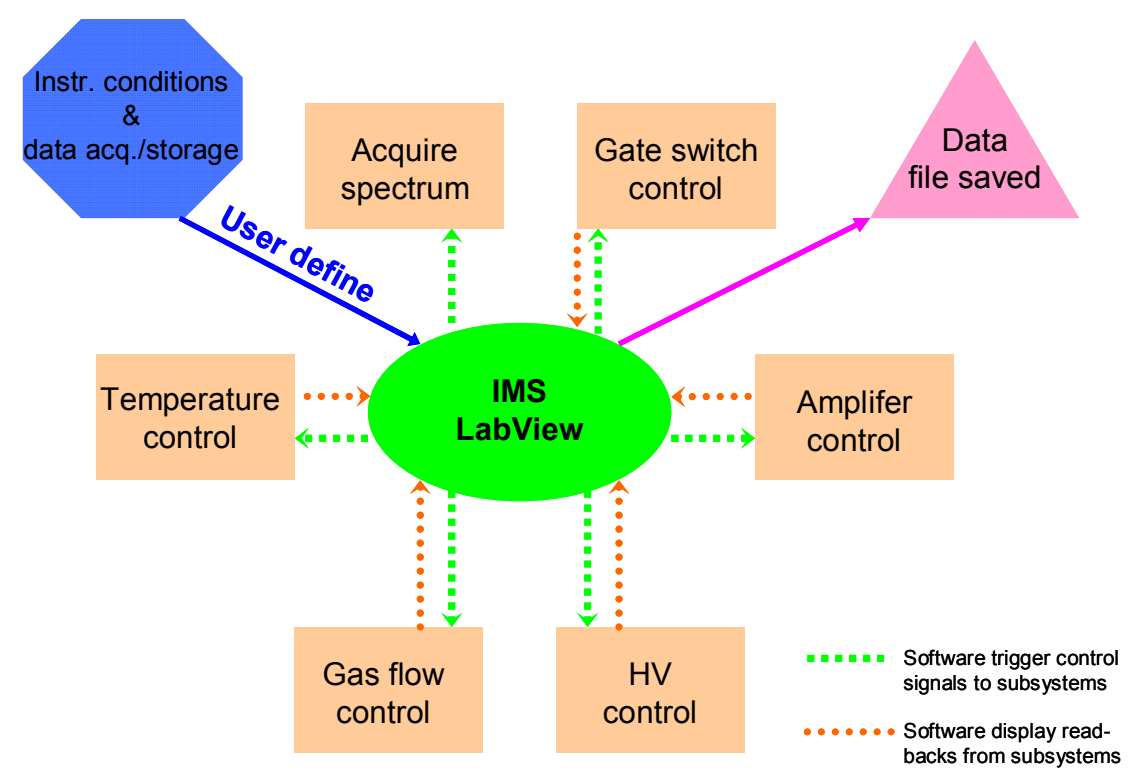

Figure 41. IMS software controls of various operating systems.

The software has multiple independent control loops, which are started and stopped in the proper sequence, and each of which control the function of one or more subsystems in the instrument. The various subsystems include temperature control and measurement, gas flow control and measurement, voltage control and measurement, control of the amplifier, timing of data acquisition, other data acquisition parameters, etc. The user is able to control instrument settings and the parameters governing data acquisition and storage via the software interface. 


\subsubsection{Signal Averaging and Data Acquisition}

Signal averaging for a single spectrum can be set to any desirable number of scans (subsamples) to improve the $\mathrm{S} / \mathrm{N}$ ratio, with a corresponding increase in total analysis time. For example, when using a scan cycle (drift time cycle) of $40 \mathrm{msec}$ and acquiring 100 scans to construct one single spectrum for signal averaging, then the total analysis time was $4 \mathrm{sec}$. If an analyte desorption profile is desired, multiple spectra are usually collected to plot a $3 \mathrm{D}$ plasmagram $(x, y, z) \rightarrow$ (drift time, signal intensity, real time from each single spectrum). Hence, if the $3 \mathrm{D}$ plasmagram required 50 spectra to be collected, then the total analysis time would be $200 \mathrm{sec}(4 \mathrm{sec} \times 50)$. Such long analysis time is not practical since analytes are usually desorbed within the first 10 to 20 seconds when the sampling substrate is introduced to the heated desorber. To achieve a reasonable number of subsamples for the studies performed, the subsample rate was set between 10 and 30 as required.

The data acquisition component of the IMS software was built to be very flexible. There were several ways to trigger the start and stop of the data acquisition when perform the IMS analysis. Triggering can be started manually by clicking the "START" button, or started at a specified date and time. Similarly, data acquisition can continue for as long as desired until the "STOP" button is pressed, until a specified time elapsed, or a specified number of spectra are acquired. Data is written to file as discussed in section 11.4.3 Data File Format below.

\subsubsection{Data File Format}

Each averaged spectrum was saved in a single text file that was exported to Microsoft Excel for data analysis. The $1^{\text {st }}$ column contains the $\mathrm{x}$-axis drift time (msec) data points, and the $2^{\text {nd }}$ column contains the $y$-axis signal intensity (DC voltage). The number of data points collected 
per scan, which can be specified by the user, normally ranged between 2000 to 3000 points. Alternatively, multiple averaged spectra can be saved in one single text file where each of the averaged spectra takes up two columns and a blank column is left in between to keep data sets separated.

\subsection{Evaluation of IMS Performance}

\subsubsection{Reactant Ion Peak Monitoring}

Once the drift tube physical components were assembled and all electrical connections were made, the first experiment performed was to evaluate the signal intensity and shape of the reactant ion peak (RIP). Operating parameters such as electrical fields, gas flow rates, temperature, moisture, gate width, and source location were varied and the IMS performance was recorded for a range of settings. The electrical fields within the IMS were divided into 4 regions, 1) the field between the ${ }^{63} \mathrm{Ni}$ source and the gate $\left(\mathrm{E}_{\mathrm{s}-\mathrm{g}}\right)$ which was varied between $100 \mathrm{~V} \cdot \mathrm{cm}^{-1}$ to $\left.300 \mathrm{~V} \cdot \mathrm{cm}^{-1}, 2\right)$ the gate field $\left(\mathrm{E}_{\mathrm{g}}\right)$ between the two gate wires which was varied between 300 $\mathrm{V} \cdot \mathrm{cm}^{-1}$ to $\left.600 \mathrm{~V} \cdot \mathrm{cm}^{-1}, 3\right)$ the drift field $\left(\mathrm{E}_{\mathrm{g}-\mathrm{a}}\right)$ between the gate and the aperture grid which was varied between $150 \mathrm{~V} \cdot \mathrm{cm}^{-1}$ to $235 \mathrm{~V} \cdot \mathrm{cm}^{-1}$, and 4) the field between the aperture grid and the detector $\left(E_{\mathrm{a}-\mathrm{d}}\right)$ which was varied between $300 \mathrm{~V} \cdot \mathrm{cm}^{-1}$ to $1000 \mathrm{~V} \cdot \mathrm{cm}^{-1}$. The gate width was varied between $100 \mu \mathrm{sec}$ to $1000 \mu \mathrm{sec}$. The peak intensity, background baseline, and drift time were recorded as each of the above conditions was varied.

\subsubsection{Evaluation of IMS ion Separation Capability}

The ion separation capabilities of an IMS are quantified using either the resolving power $\left(R_{p}\right)$ or the resolution $\left(R_{p p}\right)$. IMS resolving power has been defined as the drift time $\left(t_{d}\right)$ of the ion 
divided by a single peak's full width at half maximum height (FWHM or $\left.w_{0.5}\right)$. This relationship is given in Equation 12:

$$
R_{p}=\frac{t_{d}}{w_{0.5}}
$$

The resolution is the measure of separation between pairs of adjacent peaks, as defined in Equation 13 where $t_{d 2}$ and $t_{d 1}$ are the species' drift times, and $w_{b 1}$ and $w_{b 2}$ are the peaks' respective widths at baseline.

$$
R_{p p}=\frac{2\left(t_{d 2}-t_{d 1}\right)}{w_{b 1}+w_{b 2}}=\frac{\Delta t_{d}}{w_{b(a v g)}}
$$

Resolving power $\left(R_{p}\right)$ and resolution $\left(R_{p p}\right)$ can be related to one another by the separation factor $(\alpha)$ described in Equation 14, where $(\alpha)$ is defined for two adjacent peaks as the ratio of their corresponding drift times $\left(t_{d 2} / t_{d 1}\right)$.

$$
R_{p p}=0.589 R_{p} \frac{\alpha-1}{\alpha}
$$

A comparison of peak resolving power $\left(R_{p}\right)$ and peak to peak resolution $\left(R_{p p}\right)$ for in-house IMS instrument vs. commercial GE Itemizer 2 was performed for 2,4-DNT, 2,4,6-TNT, RDX, and PETN in negative mode, and MA, MDA, Benzoylecgonine and Cocaine in positive mode. 


\section{PART IV: RESULTS AND DISCUSSION}

\section{CHAPTER 12: DETECTION OF VOLATILE COMPONENTS OF ILLICIT DRUGS BY SPME-IMS}

CONTENT

PAGE

12.1 Plasmagrams of the newly detected compounds 136

12.2 Detection Limits, Repeatability and Linear Dynamic Range of SPME-IMS. 141

12.3 SPME Extraction Profile at Equilibrium for model compound DMNB 144

12.4 Optimization of IMS conditions using a Genetic Algorithm 146

12.5 Headspace sampling of cocaine, MDMA, and marijuana.

*Note: Section 12.1 to 12.4 have been pre-peer reviewed from a version of the following article, "Analysis of volatile components of drugs and explosives by solid phase microextraction-ion mobility spectrometry, Hanh Lai, Patricia Guerra, Monica Joshi, José R. Almirall, Journal of Separation Science 31(2): 402 - 412" Copyright Wiley-VCH Verlag GmbH \& Co. KGaA. Reproduced with permission.

\subsection{Plasmagrams of the newly detected compounds}

The compounds which were detected are methyl benzoate, piperonal, limonene, and $\alpha$ and $\beta$-pinene. Their detection with IMS is of interest because they are volatile chemical markers of cocaine, MDMA and marijuana respectively.[32, 45, 135] Methyl benzoate (MB) is more difficult to ionize regardless of the IMS polarity. MB was detected in positive mode in the absence of a dopant in this study. Finally, limonene, $\alpha$-pinene and $\beta$-pinene are very similar compounds, and hence have a propensity to be detected under the same conditions with almost the same sensitivity and similar reduced mobilities $\left(\mathrm{K}_{0}\right)$ (see Table 18) The reduced mobility values of limonene, $\alpha$-pinene and $\beta$-pinene were determined to be $1.276,1.263$, and $1.257 \mathrm{~cm}^{2} \cdot \mathrm{V}^{-}$ ${ }^{1} \cdot \mathrm{s}^{-1}$ respectively by the Smiths IonScan $400 \mathrm{~B}$. These $\mathrm{K}_{0}$ values have no significant differences, 
and so are not resolved in one single spectrum with current commercial IMS instruments (experimented using both GE Itemizer 2 IMS and Smiths IonScan IMS). The reduced mobilities of piperonal, and methyl benzoate were calculated from the GE Itemizer 2 (using the estimated drift tube length of $5 \mathrm{~cm}$ and drift electric field of $250 \mathrm{~V} \cdot \mathrm{cm}^{-1}$ ) to be 1.51 , and $1.55 \mathrm{~cm}^{2} \cdot \mathrm{V}^{-1} \cdot \mathrm{s}^{-1}$. Figure 42 and Figure 43 show the spectra obtained for these compounds with their $\mathrm{K}_{0 \text { (RIP) }}$ and $\mathrm{K}_{0 \text { (Analytes) }}$ labeled. The spectra shown were obtained using the GE IMS with one single scan at the moment when the signal produced by the product ions is at its maximum intensity rather than using the cumulative or average intensity.

Table 18. Reduced mobilities of volatile components at the corresponding operating condition.

\begin{tabular}{|c|c|c|c|c|c|c|c|}
\hline Compounds & $\begin{array}{l}\text { Molecular } \\
\text { Structure }\end{array}$ & $\mathbf{K}_{0}$ & $\frac{\text { Temp. }}{\left({ }^{\circ} \mathrm{C}\right)}$ & $\frac{\text { Drift Flow }}{\left(\mathrm{ml}^{\prime} \mathrm{min}^{-1}\right)}$ & $\frac{\text { S. Flow }}{\left(\mathrm{ml} \cdot \mathrm{min}^{-1}\right)}$ & Dopant & Mode \\
\hline Piperonal & & 1.51 & 80 & 350 & 500 & $\begin{array}{l}\text { Nicotin } \\
\text {-amide }\end{array}$ & $(+)$ \\
\hline $\begin{array}{l}\text { Methyl } \\
\text { Benzoate }\end{array}$ & & 1.55 & 190 & 250 & 1000 & Air & $(+)$ \\
\hline Limonene & & 1.26 & & & & & \\
\hline$\alpha$-Pinene & & 1.28 & 110 & 50 & 1000 & $\begin{array}{l}\text { Nicotin } \\
\text {-amide }\end{array}$ & $(+)$ \\
\hline$\beta$-Pinene & & 1.26 & & & & & \\
\hline
\end{tabular}



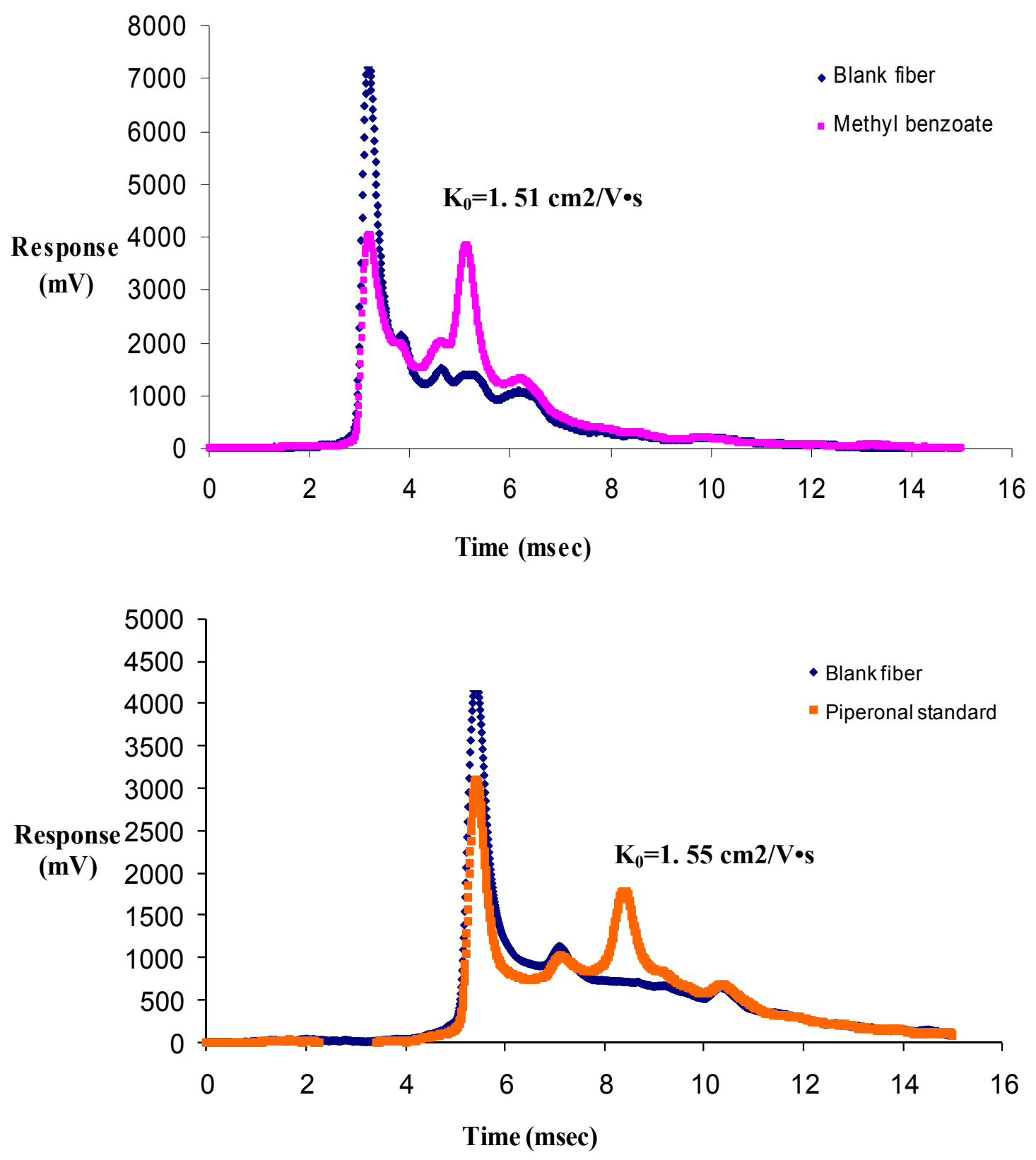

Figure 42. IMS spectra of piperonal, and methyl benzoate overlayed on blank fiber extraction at optimized operating condition. 

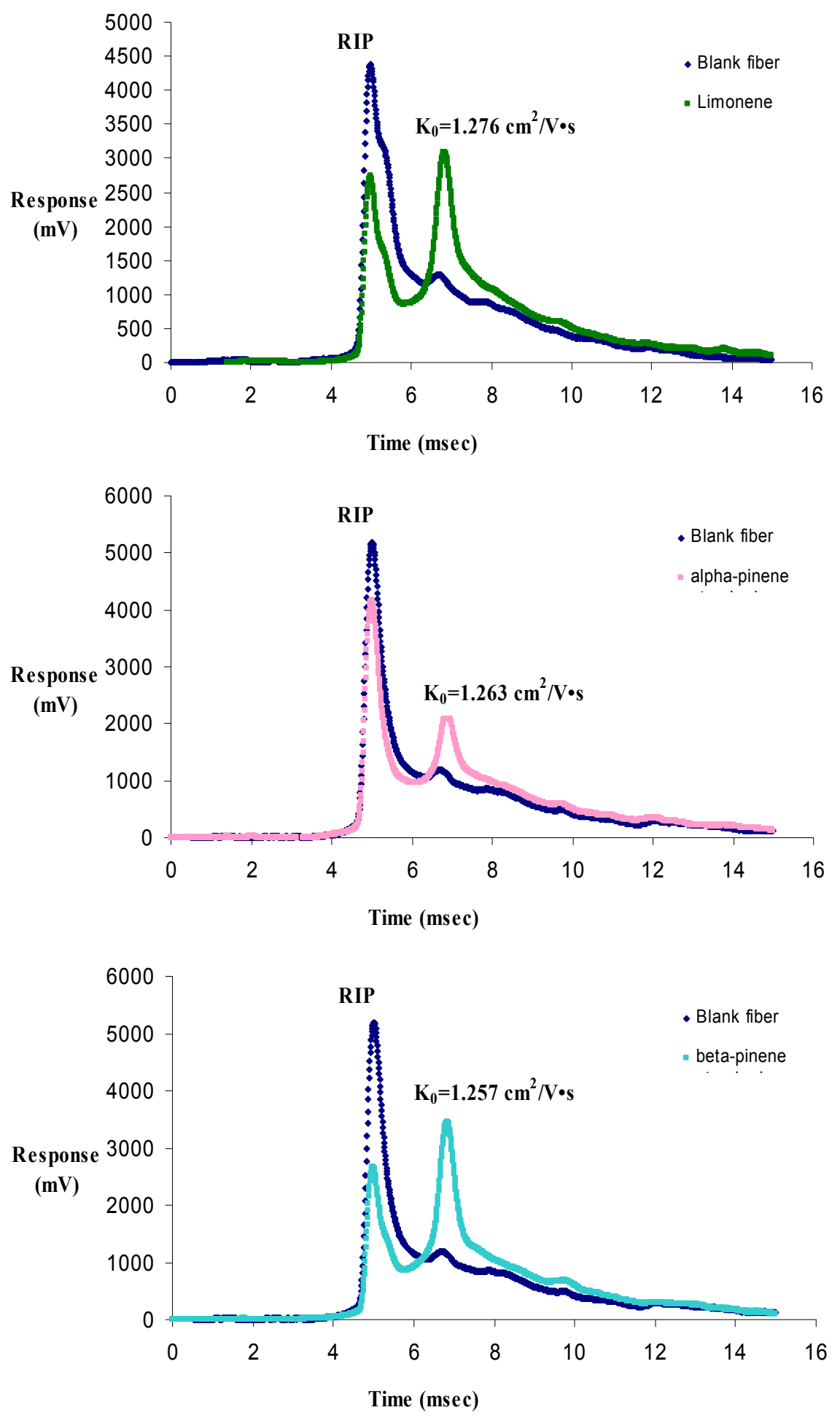

Figure 43. IMS spectra of limonene, $\alpha$-pinene, and $\beta$-pinene overlayed on blank fiber extraction at optimized condition. 
Another important finding is the formation of proton-bound dimer in the analysis of limonene, $\alpha$ - and $\beta$-pinene. Figure 44 shows the plasmagram of $\beta$-pinene as an example of such process occurring. The initial decrease in the peak intensity of the reactant ion corresponds with the formation of the protonated monomer. The appearance of a third peak and a corresponding decrease in the monomer peak is evidence of the formation of a proton-bound dimer.[17] This process is typically encountered on IMS analyzers when the amount of analyte molecules is high, as was the case in this example. The mass introduced from a SPME fiber was approximately 200 ng. This proton-bound dimer formation was only observed in SPME-IMS experiments where the analytes were pre-concentrated, but not in the direct gas sample analysis experiments.

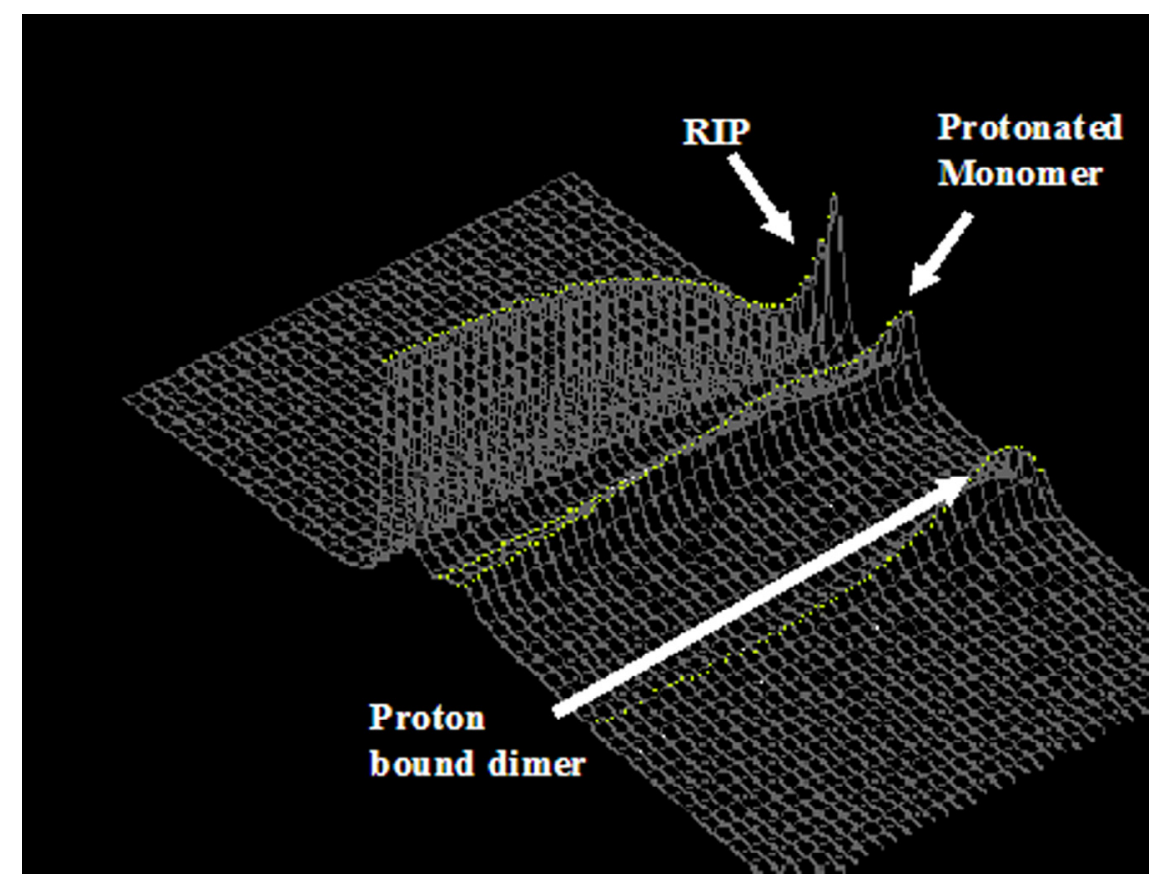

Figure 44. 3D plasmagram, plot of $\beta$-pinene's monomer and proton bound dimer. 


\subsection{Detection Limits, Repeatability and Linear Dynamic Range of SPME-IMS}

The analytes in this study were delivered to the instrument as discrete samples from solid-phase microextraction fibers and as headspace gas volume inside syringes. For this reason detection limits are expressed in mass units. The limits of detection in Table 19 compare SPMEIMS versus the stand-alone IMS for the compounds in this study, which was calculated using the response curves shown in Figure 45 and Figure 46, respectively. Low nanogram detection limits were achieved through both sampling setups. SPME-IMS detection limit for methyl benzoate and limonene were 1.4 and 4 times lower than stand-alone IMS. These results may be attributed to the ion shutter in a commercial instrument opening for only 0.1 to $0.3 \mathrm{~ms}$ to allow the ion swarms to enter the drift region.[17] Thus, SPME-IMS can achieve a lower limit of detection because the sample is pre-concentrated on the fiber and is introduced into the IMS in a tight plug, meaning higher ion density in a $20 \mathrm{~ms}$ duty cycle. This equates to higher ion flux, $\mathrm{J}$ (ions $\cdot \mathrm{cm}^{-2} \cdot \mathrm{s}^{-1}$ ) crossing the ion gate for detection. In the case of $\alpha$-pinene, the lower limit of detection is achieved by IMS direct gas injection (0.03 ng) as compared to SPME-IMS (4.29 ng). This may be the result of SPME PDMS fiber chemistry which does not favor the sharp desorption profile for $\alpha$-pinene.

Improved detection limits in an IMS system are generally the result of increases in efficiency of chemical ionization, ion's stability, and more efficient conversion of ion-molecule collisions into an activated intermediate.[17] The improvements realized via SPME-IMS are small compared to the impact of these three limiting characteristics of IMS systems. However, SPME-IMS has a pronounced effect in real case scenarios where only limited analytes are present in the headspace for sampling. Thus, the pre-concentration of an analyte on a SPME fiber increases the available mass for detection by IMS. In addition, the repeatability of the IMS response based on 25 replicates (within a 1 week period) improved from 7\% RSD for gas sampling to 3\% RSD for SPME sampling for the model compound DMNB. 

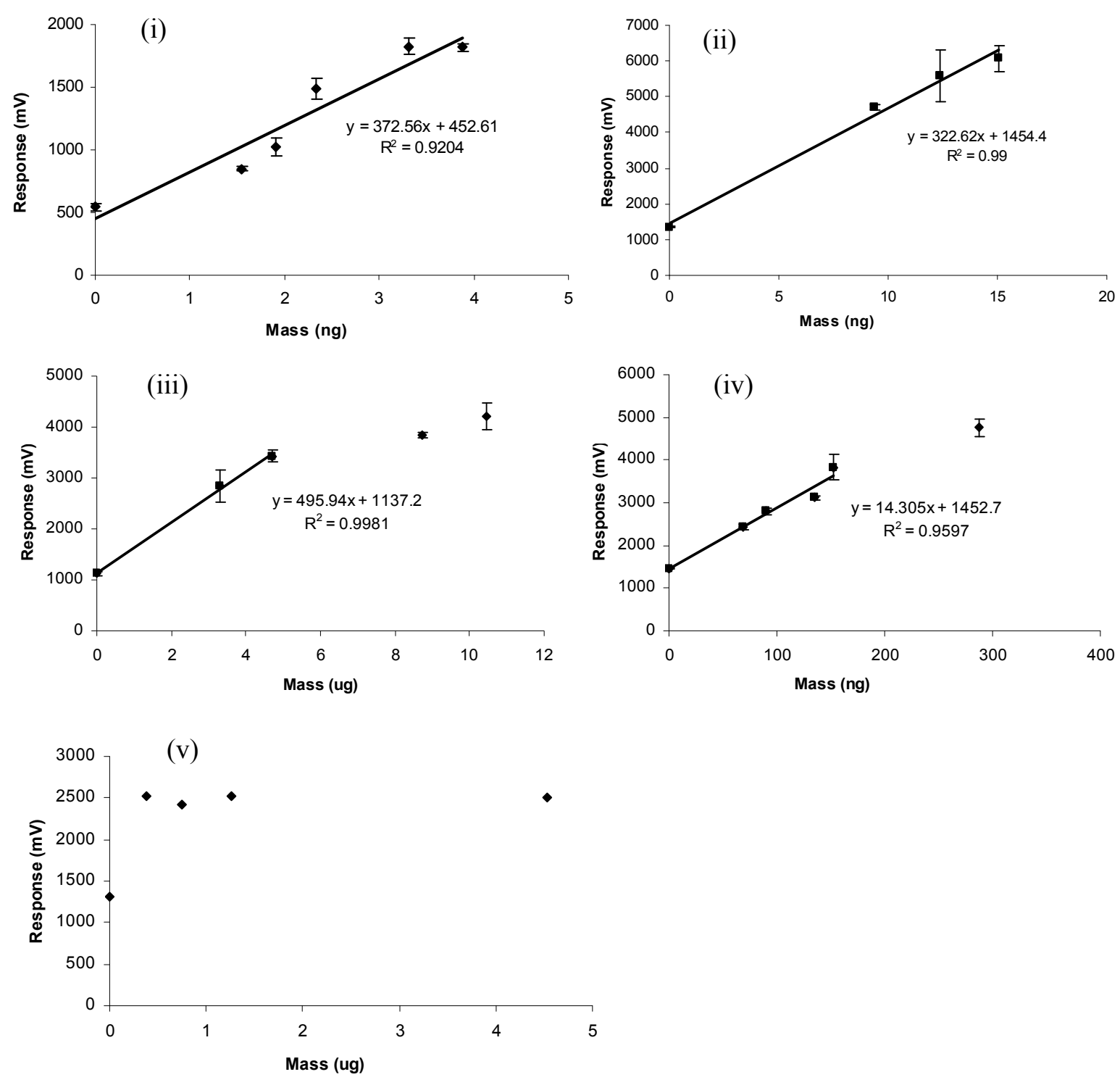

Figure 45. SPME-IMS response curve of (i) Piperonal, (ii) Methyl Benzoate, (iii) Limonene, (iv) $\alpha$-pinene, (v) $\beta$-pinene at individually optimized IMS condition. 

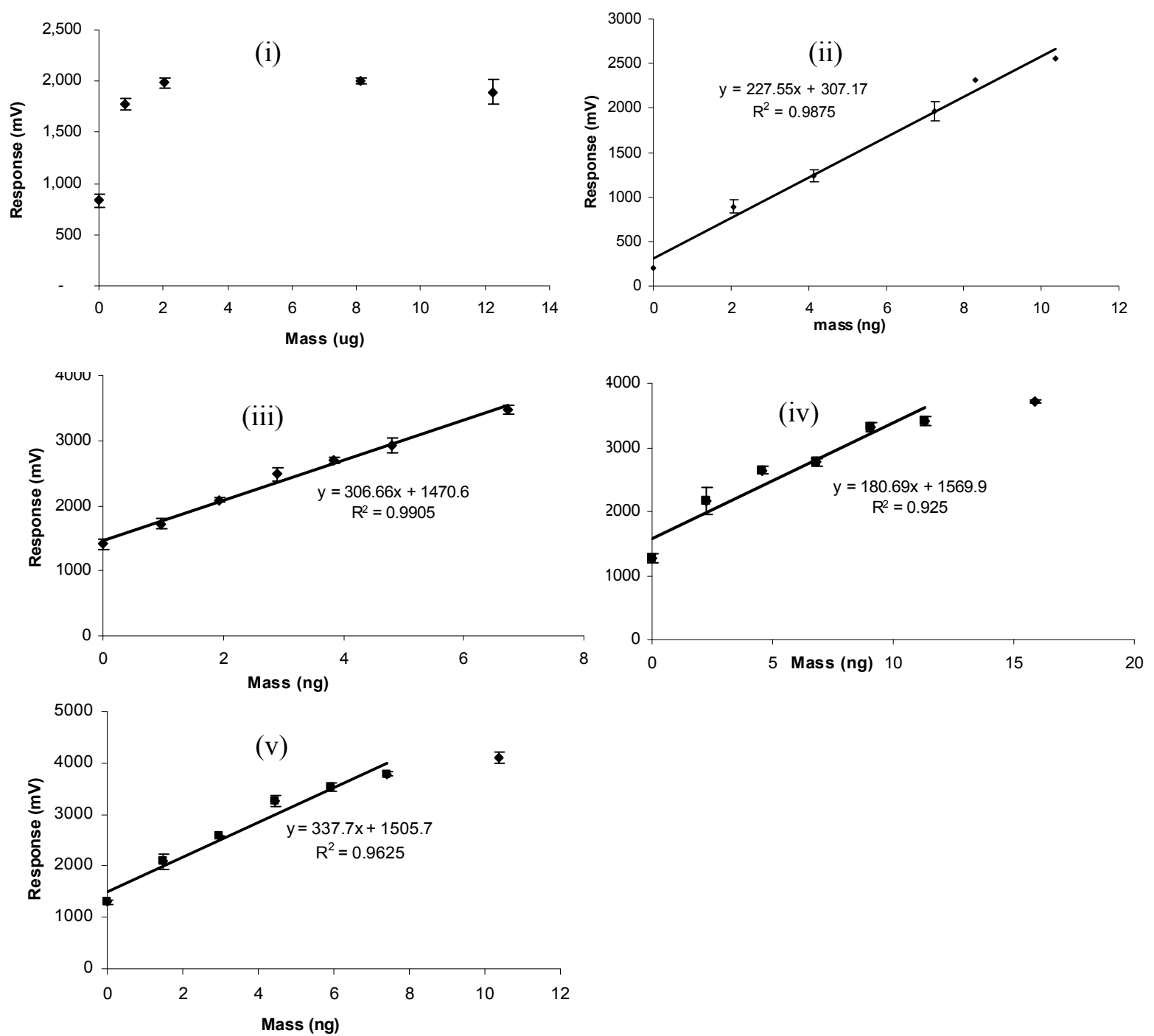

Figure 46. Response Curves by direct headspace volume injection into IMS. (i): Piperonal, (ii): Methyl Benzoate, (iii): Limonene, (iv): $\alpha$-pinene, (v): $\beta$-pinene at individually optimized IMS condition.

Table 19 reports the absolute mass of compounds introduced along with the corresponding response for each mass. Linear ranges are commonly expected to be between 10 and $100 \mathrm{ng}$ [17] with $\alpha$-pinene exhibiting slightly larger linear ranges (80-300 ng). The linear dynamic range for piperonal is expected to be significantly below $10 \mathrm{ng}$ with low amounts of piperonal saturating the detector. Because of the high concentration of piperonal in the headspace, 
to introduce less than $10 \mathrm{ng}$ of piperonal, a different experimental approach to deliver gas at very low mass loading was needed. SPME-IMS was able to achieve smaller quantity of mass loading may be explained by the minimum time required for partition to start. For the terpenes, SPME favors the extraction of the compounds onto the fiber and results in much higher mass loading. To reduce the mass extracted on the SPME fiber, a $30 \mu \mathrm{m}$ PDMS fiber can be used instead of the $100 \mu \mathrm{m}$ employed in this study.

Table 19. LODs and LDRs calculated for SPME-IMS and direct IMS from their corresponding response curve.

\begin{tabular}{|c|c|c|c|c|}
\hline Compounds & LOD by IMS & $\begin{array}{c}\text { LOD by } \\
\text { SPME-IMS }\end{array}$ & $\begin{array}{l}\text { LDR by direct } \\
\text { injection }\end{array}$ & $\begin{array}{c}\text { LDR by SPME } \\
\text { injection }\end{array}$ \\
\hline Piperonal & N/A & $0.45 \mathrm{ng}$ & NA & (1-4+) ng \\
\hline Methyl benzoate & $0.33 \mathrm{ng}$ & $0.23 \mathrm{ng}$ & $(2-10+) n g$ & $(10-15+) \mathrm{ng}$ \\
\hline Limonene & $0.57 \mathrm{ng}$ & $0.14 \mathrm{ng}$ & $(1-7+) n g$ & $(3-10+) \mu \mathrm{g}$ \\
\hline A-pinene & $0.03 \mathrm{ng}$ & $4.29 \mathrm{ng}$ & $(2-15+) n g$ & $(80-300+) \mathrm{ng}$ \\
\hline B-pinene & $0.13 \mathrm{ng}$ & N/A & $(2-11+) n g$ & N/A \\
\hline
\end{tabular}

Table 19 details the differences in both limit of detection (LOD) and linear dynamic range (LDR) for the two sampling setups. This strengthens the case for targeting high vapor pressure compounds associated with the parent explosives and drugs. The linear dynamic ranges should be similar for both the SPME-IMS set up and the stand alone IMS although this is not reflected in Table 19 due to variation in sampling ranges.

\subsection{SPME Extraction Profile at Equilibrium for model compound DMNB}

The amount (mass) of a particular compound that was extracted by the SPME is represented in Figure 47 as the IMS response in $\mathrm{mV}$ plotted against the extraction time. The minimum amount of exposure time needed, under these conditions to generate a signal equivalent to a mass of $\sim 2 \mathrm{ng}$ (instrument response of $400 \mathrm{mV}$ ) is less than 1 second for DMNB. The 
extraction curve shown in Figure 47 (i) also suggest that an equilibrium response (related to an equilibrium loading on the fiber) was reached after about 200 seconds of fiber exposure for the DMNB extraction. The equilibrium amount on the fiber was approximately $150 \mathrm{ng}$ (instrument response of $2200 \mathrm{mV}$ ) of DMNB under these conditions.
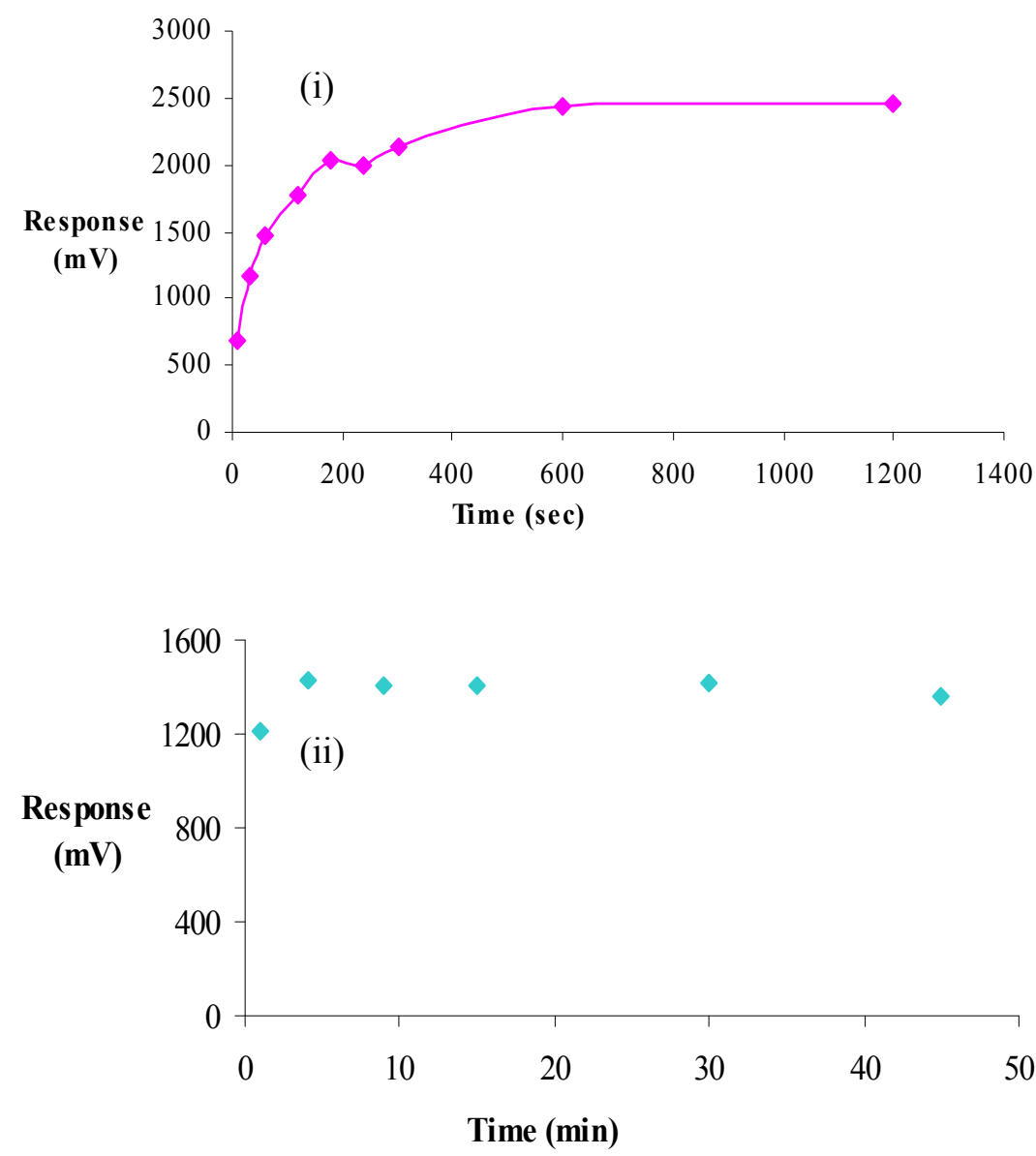

Figure 47. (i) SPME-IMS extraction equilibrium time curve, (ii) headspace equilibrium time for DMNB model compound.

These data suggest that SPME extraction and preconcentration at equilibrium can be achieved in relatively short times but it is important to bear in mind that during real-world scenarios involving large cargo containers, the time required for the container to reach headspace equilibrium will be longer than the time required for the small vial used in this study. Hence, 
unless extraction begins when the headspace concentration is already at equilibrium, otherwise extraction time can be as long as a few hours to collect sufficient mass for detection. Figure 47 (ii) illustrates the headspace equilibrium curve for DMNB developed from a $15 \mathrm{~mL}$ vial containing approximately 0.1 gram of DMNB solids. The response suggests that either the IMS saturation point is reached or the headspace equilibrium is reached almost instantaneously after the sample is sealed for DMNB $\left(\mathrm{P}_{\mathrm{vap}}=10^{-3}\right.$ torr $)$ as compared to $>30$ minutes for TNT $\left(10^{-6}\right.$ torr $)$ under the same laboratory conditions. The remaining compounds in this study have vapor pressures even higher than DMNB, and thus reached equilibrium even more quickly.

\subsection{Optimization of IMS conditions using a Genetic Algorithm}

To optimize the IMS conditions to detect the compounds in the most efficient manner, a Genetic Algorithm (GA) was used as a quick and reliable systematic optimization tool for finding optimal IMS operating conditions for the new compounds of interest in this study. Moreover, a GA programming code that is IMS specific was developed because the recognized need of many research laboratories that are trying to optimize compounds that are not currently detected by the IMS at the manufacturer's setting.

It is sometimes beneficial to perform a random search of operating parameters when trying to simply achieve a response for a compound that has not previously been detected by IMS. Random optimization, however, is risky and does not guarantee an instrument response after some effort spent in evaluating the different configurations.[130] Figure 48 (i) and (ii) compare the response of 80 configurations produced by GA versus 80 configurations generated using a randomizing code. It is evident that the GA has far less configurations with a "zero" response as well as a trend of improvement toward the later members. 


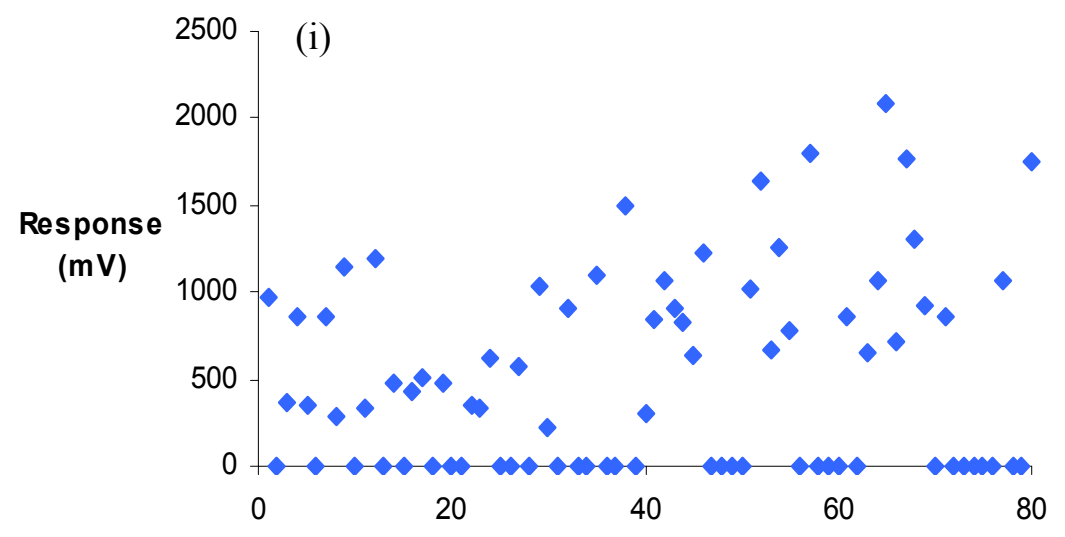

GA Member \#

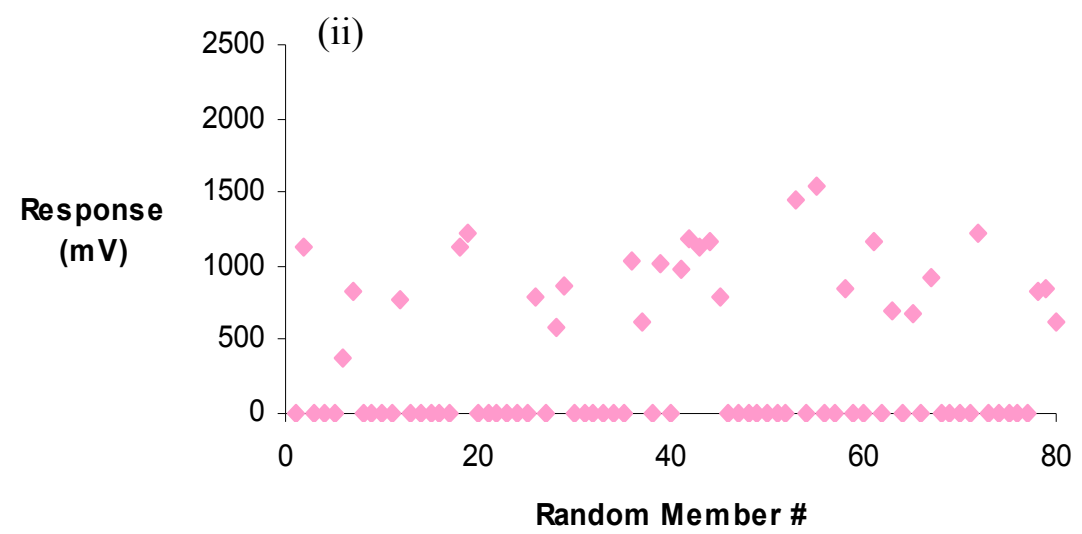

(iii)

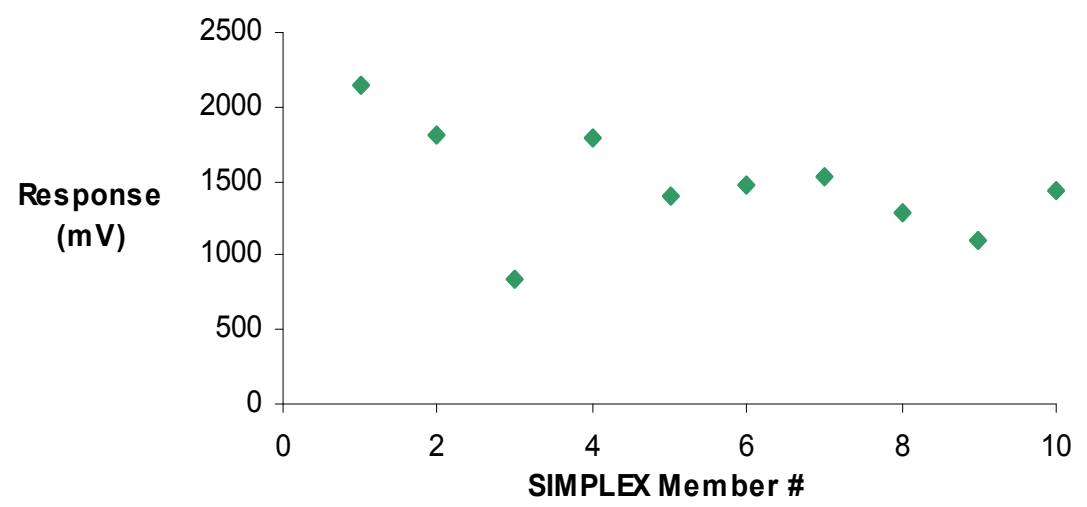

Figure 48. Comparison of IMS configurations' responses obtained by GA (i) versus random (ii), and GA as precursor to SIMPLEX (ii) for DMNB. 
Genetic algorithms (GAs) are suitable for IMS systems because GAs are effective for non-linear optimization.[128] Another reason for the selection of a GA over other optimization techniques such as SIMPLEX optimization is that GAs are less sensitive to the investigator's choice of starting point.[136, 137] This is a desired capability for the initial selection of the operating parameters for the detection of compounds where no previous information regarding the detection parameters are available. In addition, unlike SIMPLEX, GAs have built-in randomness which prevent the algorithm from being fixed at a local optimum.[128-130] SIMPLEX, on the other hand, is suitable for fine tuning the optimization of the operating parameters.[136] It is therefore recommended to first optimize the parameters using a GA to find a configuration near the global optimum, and then to use SIMPLEX to further optimize the conditions. Figure 48 (iii) illustrates the results obtained using GA as a precursor of SIMPLEX by using GA's best configuration as SIMPLEX's reference starting point.[138] The results obtained in the present study did not demonstrate the benefit of using SIMPLEX as we expected. However, this may be due to the limitation of GE IonTrack Itemiser 2 ability to finely adjust the operating parameters.

This present study considered the GA to have converged when the average responses of three consecutive generations had no significant difference. This behavior is represented in Figure 49 for the model compound DMNB after the $6^{\text {th }}$ GA generations. 


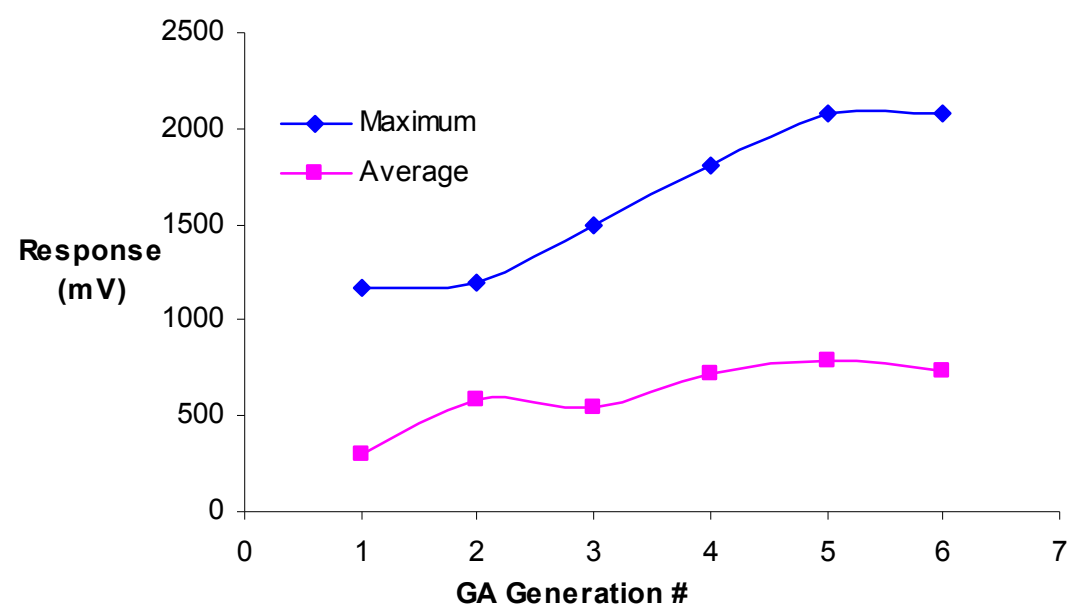

Figure 49. Convergence of GA optimization search after six generations for DMNB test compound.

\subsection{Headspace sampling of cocaine, MDMA, and marijuana}

SPME-IMS was demonstrated as a rapid and reliable trace detection technique for noninvasive headspace sampling of air inside a quart can container. Cocaine $\mathrm{HCl}$ and free base, MDMA tablets, and marijuana samples were successfully detected for the first time using IMS by means of headspace sampling, with detection algorithms based on the volatile chemical markers associated with the targeted drugs. Figure 50 (i) shows the response for a 5-minute SPME extraction in the headspace of a $15 \mathrm{~mL}$ glass vial containing $0.05 \mathrm{~g}$ of cocaine $\mathrm{HCl}$. The compound detected was methyl benzoate, presumptively based on the peak with a drift time of $5.23 \mathrm{msec}$ (reduced mobility $\left(\mathrm{K}_{0}\right)=1.55 \mathrm{~cm}^{2} \cdot \mathrm{V}^{-1} \cdot \mathrm{s}^{-1}$ in air). When SPME extraction was performed for 30 minutes or more, an additional peak at drift time of $6.75 \mathrm{msec}\left(\mathrm{K}_{0}=1.20 \mathrm{~cm}^{2} \cdot \mathrm{V}^{-}\right.$ ${ }^{1} \cdot \mathrm{s}^{-1}$ in air) was observed. This additional peak is not the result of the proton-bound dimer from the first compound because the peak rises simultaneously with the first peak as shown in Figure 51. 

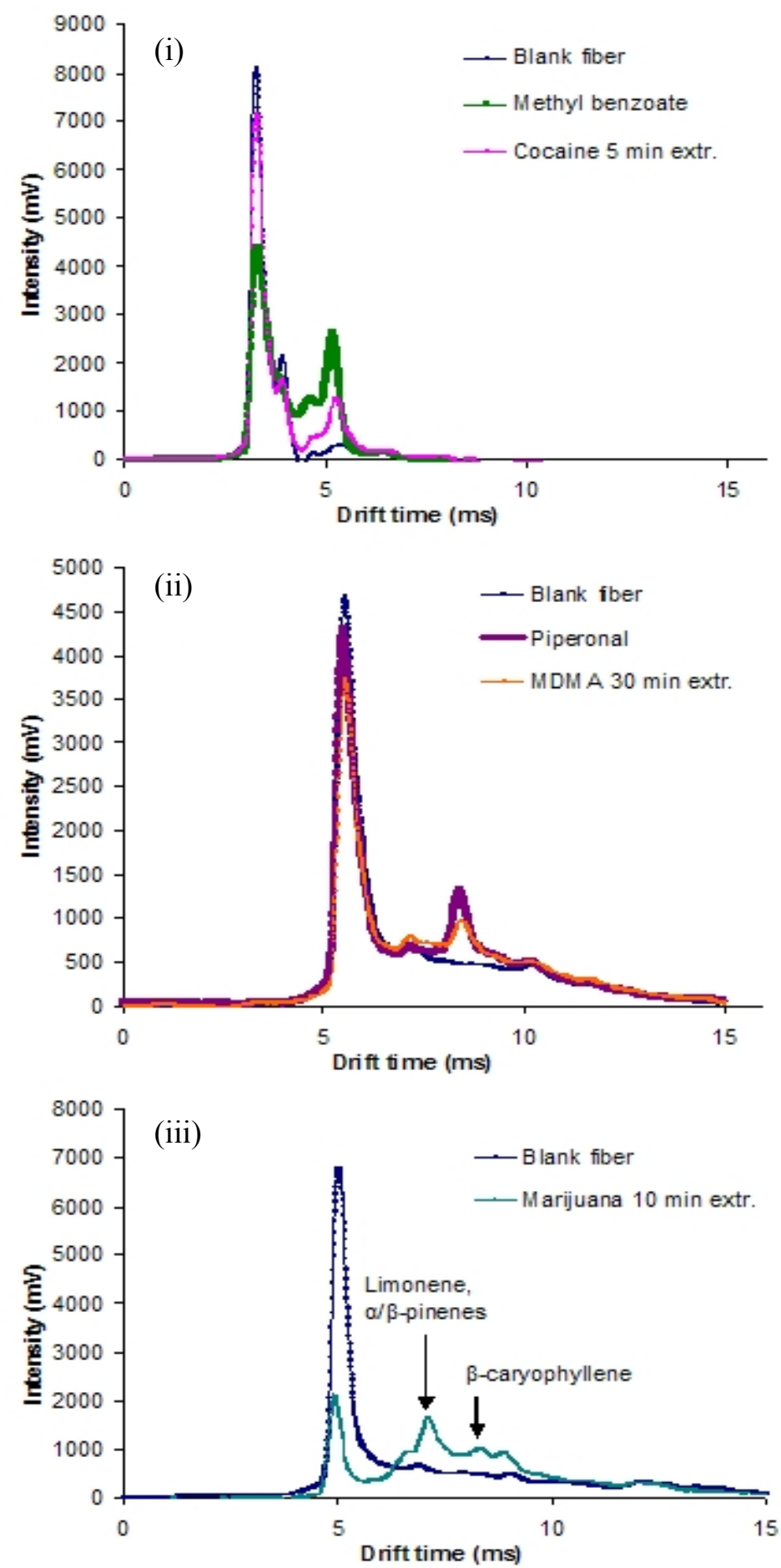

Figure 50. Overlay spectrum of a five-minute SPME headspace extraction of standards vs. actual drugs; (i) cocaine $\mathrm{HCl}$, (ii) MDMA, and (iii) marijuana. 


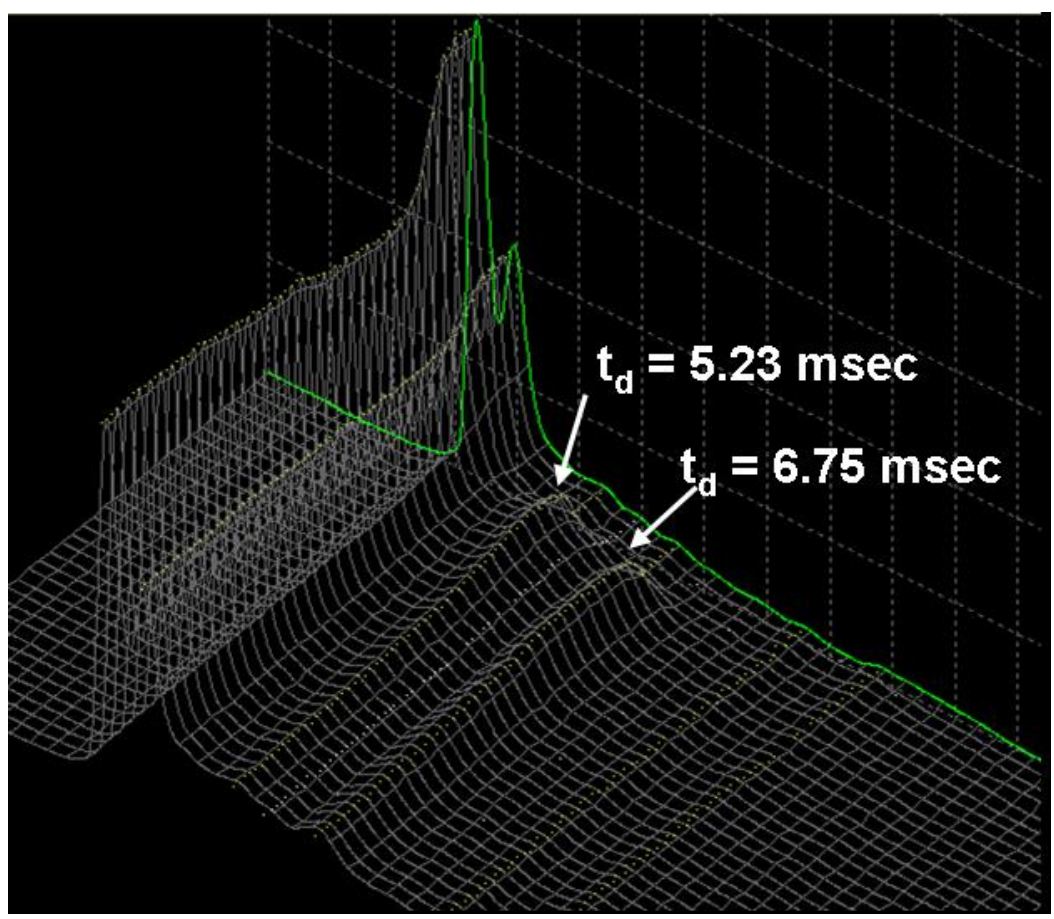

Figure 51. 3D plasmagram of a 30-minute SPME headspace extraction of cocaine $\mathrm{HCl}$.

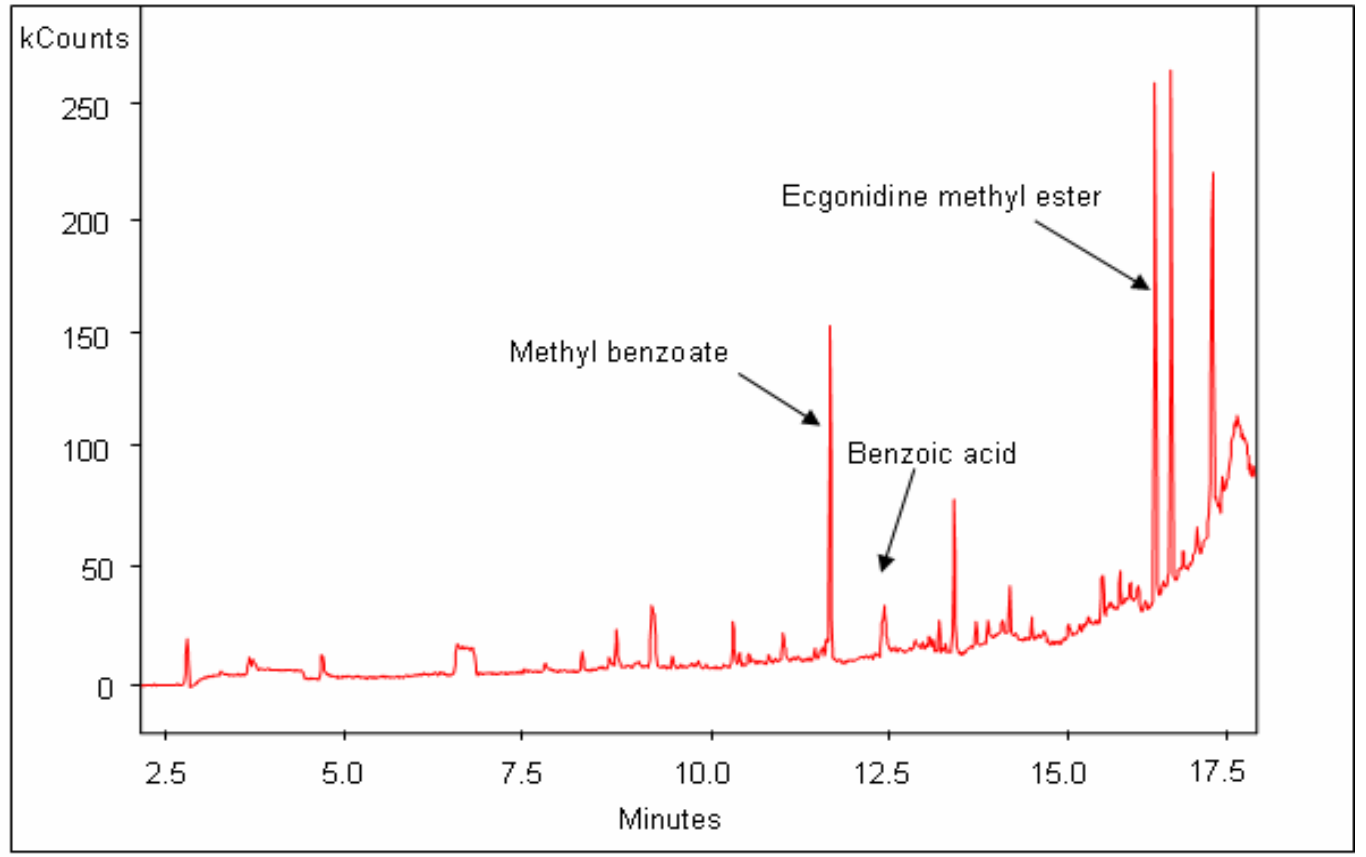

Figure 52. Gas chromatogram of a 30-minute SPME extraction of cocaine $\mathrm{HCl}$ sample inside a glass vial. 
The chromatogram shown in Figure 52 was obtained from a duplicate SPME extraction of 30 minutes on the same sample indicated that in addition to methyl benzoate, ecgonidine methyl ester (EDME) was also extracted onto the fiber. The formation of these two decomposition compounds is shown in Figure 53. The vapor pressure of EDME is reported to be 5 orders of magnitude greater than the vapor pressure of cocaine, [51] ensuring that it is readily present in the headspace. Although methyl benzoate has a higher vapor pressure than EDME, EDME consistently gave higher response in GC/MS for extraction of more than 30 minutes. The formation of methyl benzoate requires two steps; the hydrolysis of both ester groups to form benzoic acid and methanol (as shown in path a of Figure 53) followed by the acid catalyzed esterification of benzoic acid and methanol to form methyl benzoate.[50] The formation of EDME is more thermodynamically favored as it requires only the single elimination of benzoic acid as shown in path $\mathbf{b}$ of Figure 53.

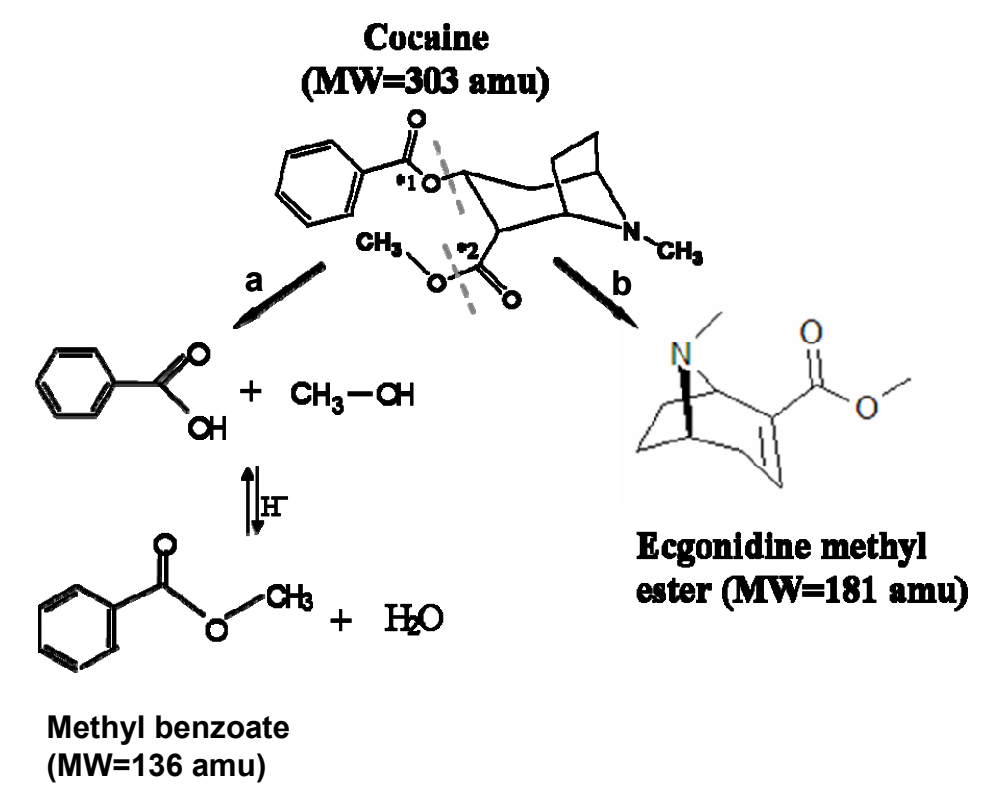

Figure 53. Formation of methyl benzoate (path a) and ecgonidine methyl ester (path b) from cocaine decomposition. 
EDME has been reported from air sampling of cocaine followed by detection using an IMS operated in the positive mode at the manufacturer's settings.[139] The simultaneous detection of both methyl benzoate and EDME by IMS, however, has not been previously reported. The methyl benzoate product ion can only be formed along with the EDME product ion under optimized conditions in air. The exact identity of the product ions formed must be confirmed using an IMS-MS,[140] work that is ongoing in the Almirall laboratory.

The detection of volatile chemical markers present in the headspace of a $50 \mathrm{~mL}$ glass vial containing $0.94 \mathrm{~g}$ MDMA (4 tablets) is illustrated in Figure 50 (ii) for a 30 minute SPME extraction. The compound detected was piperonal at a drift time of $8.45 \mathrm{msec}\left(\mathrm{K}_{0}=1.51 \mathrm{~cm}^{2} \cdot \mathrm{V}^{-}\right.$ ${ }^{1} \cdot \mathrm{s}^{-1}$ in nicotinamide). The compounds extracted on the SPME fiber were confirmed by GC/MS to be piperonal, methyl piperonal ketone, and methylenedioxyphenylacetic acid.

Marijuana contains a large variety of volatile organic compounds resulting in very strong odors composed of at least 5 major volatile compounds dominating the sample headspace. The most abundant compounds in the headspace are identified as limonene, $\alpha / \beta$-pinene, $\beta$-myrcene, $\beta$ ocimene, and $\beta$-caryophyllene.[141] These compounds serve as excellent potential volatile chemical markers for the detection of marijuana by SPME-IMS. A 5 minute SPME headspace extraction of the quart can containing 5 grams of marijuana sample yielded a set of peaks, shown in the spectrum Figure 50 (iii), which can be used as multiple detection channels. The first product ion peak at the average drift time of $7.15 \mathrm{msec}$ corresponds to limonene, $\alpha / \beta$-pinene, and $\beta$-myrcene ( $\mathrm{K}_{0}$ between 1.26 and $1.28 \mathrm{~cm}^{2} \cdot \mathrm{V}^{-1} \cdot \mathrm{s}^{-1}$ in nicotinamide). The product ions from these compounds have very similar reduced mobility values $\left(\mathrm{K}_{0}\right)$, and therefore cannot be resolved with current IMS resolutions. Literature also reported the reduced mobilities of limonene, $\alpha$-pinene to be the same $\left(1.66 \mathrm{~cm}^{2} \cdot \mathrm{V}^{-1} \cdot \mathrm{s}^{-1}\right.$ in air).[142] The peak at drift time of $8.33 \mathrm{msec}\left(\mathrm{K}_{0}=1.10 \mathrm{~cm}^{2} \cdot \mathrm{V}^{-}\right.$ 
${ }^{1} \cdot \mathrm{s}^{-1}$ in nicotinamide) was determined to be $\beta$-caryophyllene, confirmed both by using single component standards in the IMS and by identification using GC/MS. The SPME-GC/MS analysis indicated $\beta$-linalool, cis- $\beta$-terpineol, and trans-2-pinanol were also present on the fiber.

Current commercial IMS detection algorithms are based on one single peak detection channel, which is acceptable for explosive detection in negative operating mode where false positives are less common (and produce less of an operational problem) than false negatives. However, detection of illicit drugs by IMS has a much higher false positive alarm rate due to the ease of ionization of gaseous molecules in the positive mode. The rate of false positive alarms can be reduced for IMS (positive ionization mode) if the detection algorithms are based on multiple target peaks. Multiple channel detection algorithms can be implemented for cocaine and marijuana detection since the headspace of these two illicit substances contains 2 or more components which were ionizeable and detectable by IMS. In the case of cocaine, two headspace components that produce peaks at drift times of $5.23 \mathrm{msec}\left(\mathrm{K}_{0}=1.55 \mathrm{~cm}^{2} \cdot \mathrm{V}^{-1} \cdot \mathrm{s}^{-1}\right.$ in air $)$ and 6.75 $\operatorname{msec}\left(\mathrm{K}_{0}=1.20 \mathrm{~cm}^{2} \cdot \mathrm{V}^{-1} \cdot \mathrm{s}^{-1}\right.$ in air $)$ can be programmed for the positive alarm of cocaine. Similarly, detection of marijuana can be programmed using two sets of marker compounds at peak drift times of $7.15 \mathrm{msec}\left(\mathrm{K}_{0}=1.27 \mathrm{~cm}^{2} \cdot \mathrm{V}^{-1} \cdot \mathrm{s}^{-1}\right.$ in nicotinamide $)$ and $8.33 \mathrm{msec}\left(\mathrm{K}_{0}=1.10\right.$ $\mathrm{cm}^{2} \cdot \mathrm{V}^{-1} \cdot \mathrm{s}^{-1}$ in nicotinamide).

\subsubsection{SPME extraction time (mass) for positive alarm}

Shown on Figure 54 (i), (ii) and (iii) are the SPME extraction time profiles for cocaine, MDMA, and marijuana samples, respectively. Marijuana quickly reached the equilibrium extraction time after 10 minutes. After this equilibrium time, extended SPME sampling time no longer increased the intensity significantly. On the other hand, cocaine and MDMA took much longer to achieve SPME extraction time equilibrium, 0.5 hour and 2.5 hour, respectively. It was 
also observed that when MDMA sampling time was greater than 2.5 hours, proton-bound dimers started to form as the result of the excess piperonal molecules available. The masses of analytes labeled next to each data points correspond to each of the extraction times. After a 2 minute SPME headspace extraction of the quart can containing $0.5 \mathrm{~g}$ of cocaine $\mathrm{HCl}$, the fiber recovered $1.50 \mathrm{ng}$ of methyl benzoate for IMS analysis. After a 1 minute SPME headspace extraction of the quart can containing $5 \mathrm{~g}$ of marijuana sample, the fiber recovered a total mass of $1.26 \mathrm{ng}$ for limonene, $\alpha / \beta$-pinene, and $\beta$-myrcene. These mass loadings on the fibers are well above the reported limits of detection for IMS. Sampling times of 1 and 2 minutes were sufficient for the detection of marijuana and cocaine volatile markers. However, in the case of MDMA, a 10 minute SPME headspace extraction of the $50 \mathrm{~mL}$ glass vial containing $0.94 \mathrm{~g}$ of MDMA (4 tablets) only recovered $0.49 \mathrm{ng}$ of piperonal, which is close to the limit of detection for IMS reported for this compound.

Therefore, to extract sufficient mass of analytes for successful detection of illicit drugs in cargo containers without impeding the throughput of the inspection process, further improvement in SPME extraction efficiency is necessary to further reduce the sampling time to a maximum of 1 minute. Furthermore, air sampling in standard cargo containers is expected to be much more challenging because of the large volume, which increases the time required for the headspace concentration to reach equilibrium. Therefore, instead of a 2-day seal, a standard cargo container may take longer, depending on air circulation and temperature, to allow for the concentrations of volatile components to reach equilibrium in the headspace. 

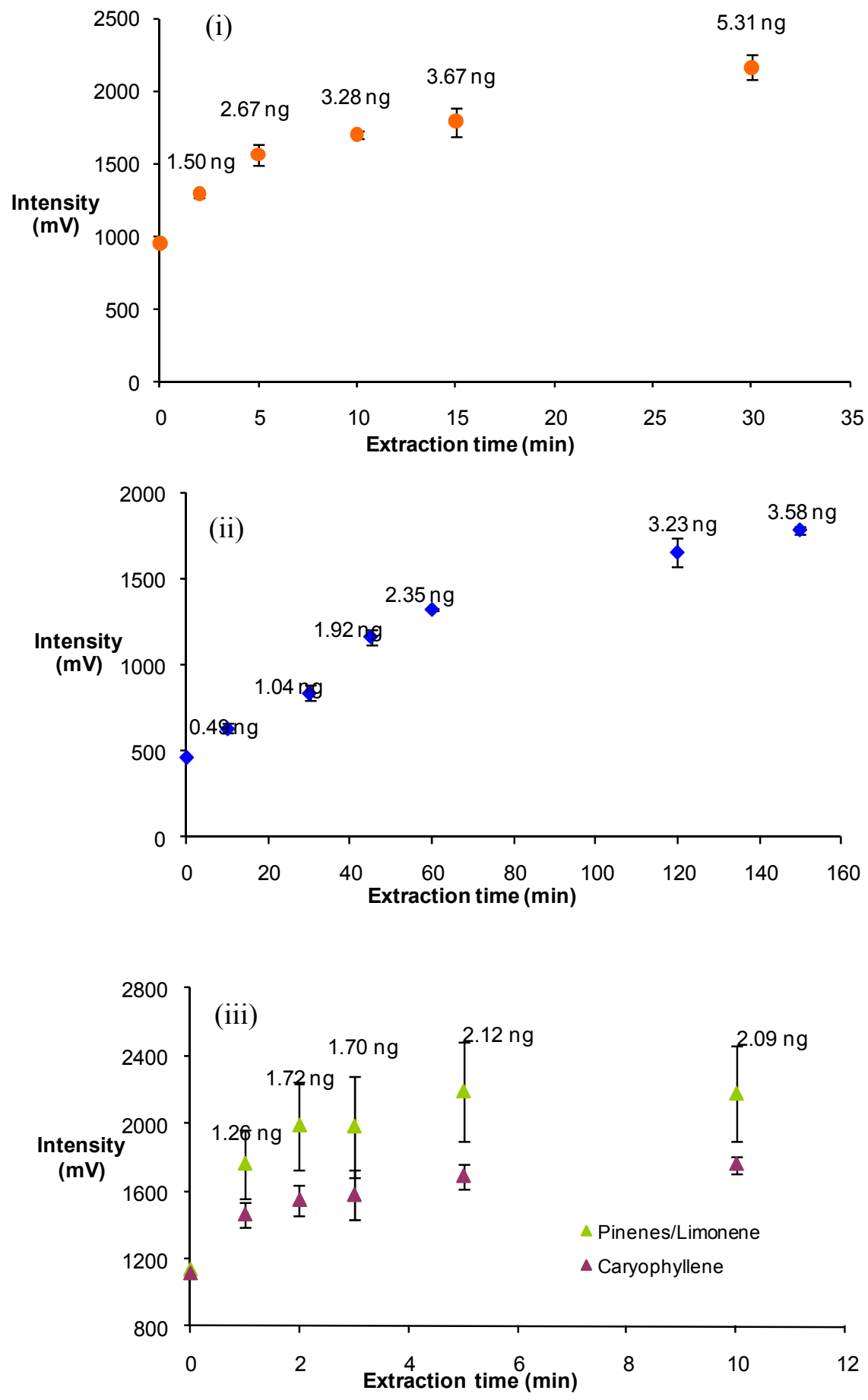

Figure 54. SPME-IMS extraction time profiles for (i) cocaine, (ii) MDMA, and (iii) marijuana. Compounds detected are methyl benzoate, piperonal and terpenes/caryophyllene, respectively at individually optimized condition. 


\subsubsection{Interference and Blind Studies}

Table 20, Table 21, and Table 22 summarize the results obtained from the interference studies of cocaine, MDMA and marijuana, respectively. The first set of experiments was performed with cans containing only the potentially interfering household consumable items. The second set of experiments was performed with cans containing a mixture of the consumable items and drug samples. Of the 7 potential interference products tested, 4 products do not contain volatile components that can be adsorbed / absorbed onto the SPME fiber or can be ionized and detected in the IMS at the settings for cocaine detection. Oregano and black pepper contained volatile component(s) that ionized and formed peaks at different drift times away from the cocaine target peak (methyl benzoate). Green tea produced two peaks, one of which was at a similar drift time as one of the cocaine target peaks (5.45 msec calibrated using MB standard on the day of the experiment). Hence, if the cocaine detection algorithm is solely based on the methyl benzoate peak, then green tea can initiate a false positive alarm.

Table 20. Location of the interference peaks with respect to methyl benzoate for samples containing cocaine and interference product.

\begin{tabular}{|c|c|c|}
\hline Household Items & Without Cocaine & With Cocaine \\
\hline Green tea leaves & $\begin{array}{l}2096 @ 5.120 \mathrm{msec} \\
1550 @ 5.435 \mathrm{msec}\end{array}$ & $\begin{array}{l}2652 @ 5.105 \mathrm{msec} \\
2588 @ 5.456 \mathrm{msec}\end{array}$ \\
\hline Rosemary & NONE & $2099 @$ \\
\hline Oregano & $1682 @ 5.678 \mathrm{msec}$ & N/A \\
\hline Hemp rope & NONE & 2129@ \\
\hline Dried mushroom & NONE & $2103 @$ \\
\hline Sesame seeds & NONE & 2256@ \\
\hline Black pepper & $2049 @ 6.688 \mathrm{msec}$ & $\begin{array}{l}2080 @ “ \\
2512 @ 6.737 \mathrm{msec}\end{array}$ \\
\hline
\end{tabular}


Table 21. Location of the interference peaks with respect to piperonal for samples containing MDMA and interference product.

\begin{tabular}{|c|c|c|}
\hline Household Items & Without MDMA & With MDMA \\
\hline Green tea leaves & $1470 @ 7.338 \mathrm{msec}$ & $\begin{array}{l}1849 @ 7.369 \mathrm{msec} \\
1056 @ 8.577 \mathrm{msec}\end{array}$ \\
\hline Rosemary & NONE & $1347 @ \quad “$ \\
\hline Oregano & $1741 @ 7.860 \mathrm{msec}$ & $\begin{array}{l}1387 @ 7.900 \mathrm{msec} \\
1353 @ 8.577 \mathrm{msec}\end{array}$ \\
\hline Hemp rope & NONE & $922 @ \quad “$ \\
\hline Dried mushroom & $1141 @ 7.081 \mathrm{msec}$ & 1294@ \\
\hline Sesame seeds & $1310 @ 7.351 \mathrm{msec}$ & $1361 @$ \\
\hline Black pepper & NONE & $1300 @$ \\
\hline
\end{tabular}

At the IMS setting for MDMA detection, 4 of the 7 tested household consumable items produced ion peaks, but none at a similar drift time as the MDMA target peak (piperonal).

Therefore, the 7 items tested pose no risks of false positive or negative alarms. In most mixtures, the piperonal peak intensity response was approximately $1300 \mathrm{mV}$, except for the can containing the mixture of MDMA and hemp (piperonal detected $922 \mathrm{mV}$ ), and the can containing the mixture of MDMA and green tea (piperonal detected $1056 \mathrm{mV}$ ). In the case of the MDMA / green tea mixture, the decrease in signal intensity may be as the result of the presence of the green tea volatile component(s) capable of forming ions by competing with piperonal for the reactant ions at the ionization source. In the case of the MDMA / hemp mixture, there was a decrease in signal intensity even when there were no additional ion swarms formed. This phenomenon may be the result of SPME site competition rather than ionization competition.

Finally, at the IMS setting for marijuana detection, 4 of the 7 tested items produced ion peaks. Similar to the MDMA study, no peaks interfered with the locations of the two targeted peaks of marijuana (limonene, $\alpha / \beta$-pinene, and $\beta$-myrcene), and $\beta$-caryophyllene. 
Table 22. Location of the interference peaks with respect to (limonene, $\alpha / \beta$-pinene) for samples containing marijuana and interference product..

\begin{tabular}{|c|c|c|}
\hline Household Items & Without Marijuana & With Marijuana \\
\hline Green tea leaves & $1526 @ 6.803 \mathrm{msec}$ & $\begin{array}{l}1141 @ 6.775 \mathrm{msec} \\
3116 @ 7.149 \mathrm{msec}\end{array}$ \\
\hline Rosemary & $1358 @ 6.825 \mathrm{msec}$ & $\begin{array}{l}1209 @ 6.774 \mathrm{msec} \\
3322 @ 7.149 \mathrm{msec}\end{array}$ \\
\hline Oregano & $1283 @ 7.263 \mathrm{msec}$ & $3003 @ \quad “$ \\
\hline Hemp rope & NONE & $3199 @$ \\
\hline Dried mushroom & NONE & $3281 @$ \\
\hline Sesame seeds & NONE & $3138 @$ \\
\hline Black pepper & $2280 @ 8.306$ msec & N/A \\
\hline
\end{tabular}

The blind study of MDMA tablets was conducted where 10 cans where prepared unknown to the analyst, and headspace SPME sampling was performed the next day for a 30 minute extraction. The results, shown in Figure 55, were positive alarms for cans A, B, and C. These were labeled as containing MDMA based on the positive peak detected at the drift time corresponding to the product ion peak of piperonal. Cans D, E, F, G, H, I, and J did not produce a product ion peak at the piperonal corresponding drift time, and were thus labeled negative for MDMA. The detection results were later compared with known results and confirmed to be correctly alarmed. 


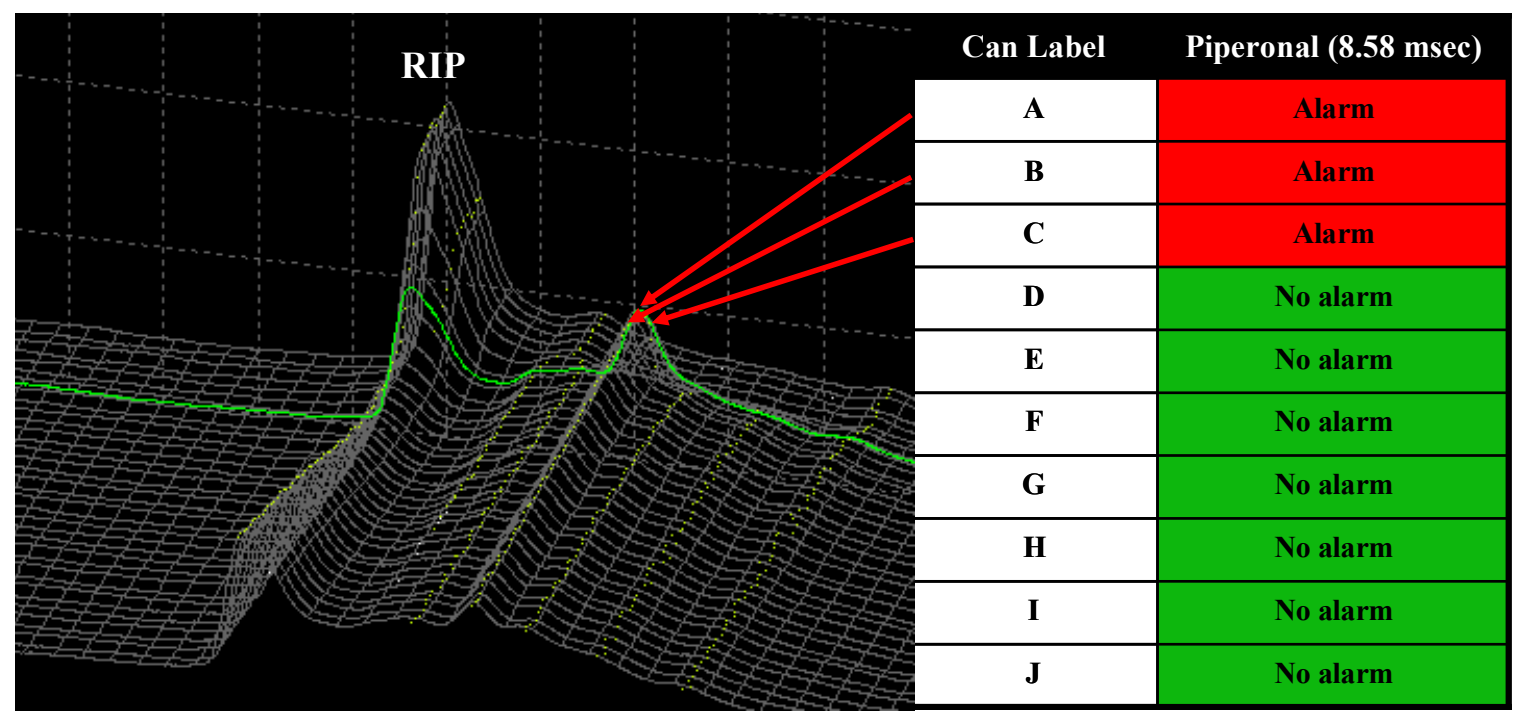

Figure 55. Piperonal alarm result for blind study of MDMA and interference products.

The results obtained from the interference and the blind studies are considered preliminary. Since the IMS settings used were newly optimized for the volatile signature compounds of the drugs in studied, further interference studies with a more enhanced list of commodities should be tested. In addition, a blind study should also be performed on cocaine and marijuana when sampling opportunities become available. 


\section{CHAPTER 13: DETECTION OF VOLATILE COMPONENTS OF EXPLOSIVES BY SPME-IMS}

CONTENT

PAGE

13.1 Headspace SPME-GC/MS analysis of Detasheet, Semtex H, and C-4 ..................162

13.2 Headspace SPME-IMS analysis of Detasheet, Semtex H, and C-4 168

\section{Suspected military explosive}

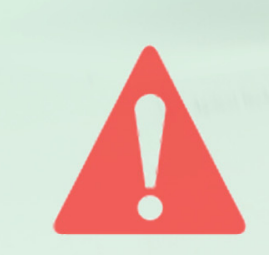

Detasheet explosive

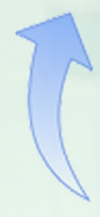

IMS detection

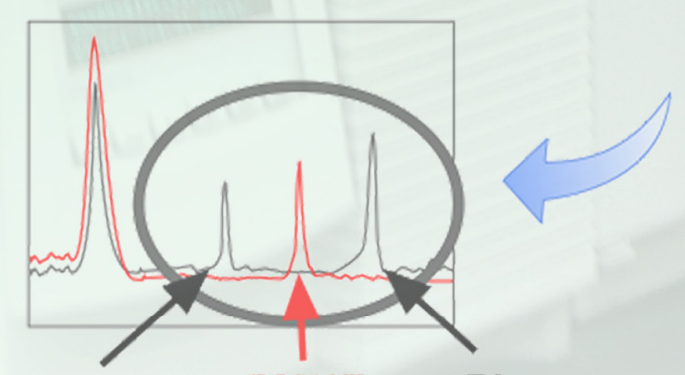

Monomer

n-Butyl acetate

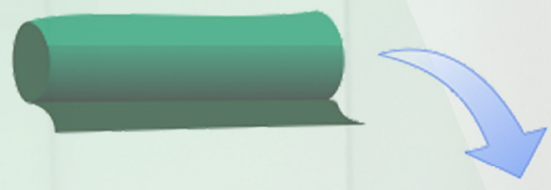

\section{SPME sampling}


The following sections present results on the detection of plastic explosives by IMS. Data are shown for the SPME extraction and identification using GC/MS, followed by detection using IMS at new optimal operating conditions for the Detasheet, Semtex H, and C-4 from different sources.

\subsection{Headspace SPME-GC/MS analysis of Detasheet, Semtex H, and C-4}

In order to target the non-energetic signatures from plastic explosives for headspace air sampling, SPME fibers were used to extract and preconcentrate analytes that are readily present in the headspace of the explosive samples under atmospheric conditions, followed by thermal desorption into a GC/MS. Signature compounds are those that can be associated with the target compound of interest, such as a degradation product, a starting material or an impurity. There are some considerations when choosing a signature to presumptively detect a target substance. The signature compounds must be differentiated from the background and present in high enough concentration to be detected by the analytical instrument.

For untagged Flex X explosives, the results in Figure 56 show (1) acetic acid, (2) 1butanol, (3) toluene, and (4) n-butyl acetate as being the most dominant peaks detected by SPMEGC/MS. Analysis of tagged Primasheet 1000 results in the detection of 3 compounds (acetic acid, toluene, and 1-butanol), along with (5) DMNB as the taggant, however, n-butyl acetate was not detectable and only a very small peak for toluene was detected. 


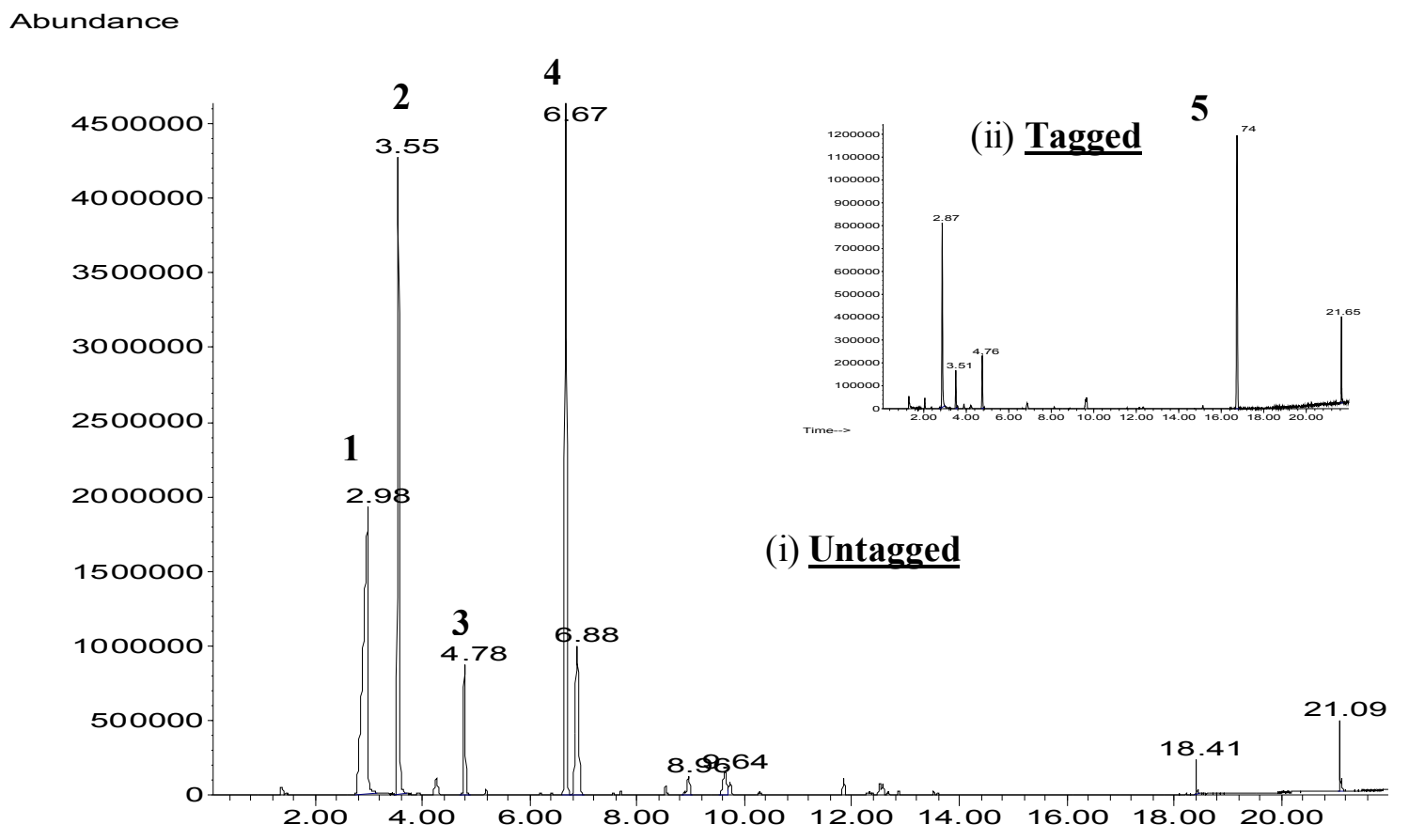

Time-->

Figure 56. Chromatogram of (i) untagged Detasheet, and (ii) tagged Primasheet explosives. (1) acetic acid; (2) 1-butanol; (3) toluene; (4) n-butyl acetate; (5) taggant DMNB.

It is possible that when the DMNB taggant is present, it competes with other trace volatile components in the headspace of the explosive. An experiment was performed to confirm the competition theory. Neat standards (to avoid solvent effects) of DMNB, and n-butyl acetate were placed into a gallon-sized metal can until headspace equilibrium was reached and extracted using fiber SPME followed by GC/MS analysis. For a short extraction time (1 min), the intensity of n-butyl acetate was 3 times higher than that of DMNB. While for a longer extraction time (20 min, as in the case of the real explosive study), the DMNB intensity was 3 times higher than nbutyl acetate. These observations suggest that, the n-butyl acetate may be present in the tagged Primasheet explosive in very low concentrations and not detectable when DMNB displacement effects occur. Although toluene were present in all Detasheet explosive samples, it was not chosen because of its common use as industrial solvent therefore provide little discrimination power as a signature compound.[143, 144] Thus, in the cases where Detasheet explosives were 
manufactured without the addition of the DMNB taggant,[17] an analytical technique such as IMS can aim to detect n-butyl acetate. Future experiments should also investigate Detasheet explosive samples that were manufactured more recently but without taggant added in order to determine if $n$-butyl acetate is introduced in the explosive from the manufacturing process or formed from the reaction between acetic acid and 1-butanol as the explosive sample ages.

For Semtex H explosives, samples from case 1 and 2 showed acetone, undecane, dodecane, and DMNB as the 4 most dominant peaks extracted and detected by GC/MS. In case 3, undecane and dodecane were not present, and could be eliminated as possible candidate signatures for Semtex H explosive. Case 3 was from a different lot of explosive than case 1 and case 2 . Although acetone was consistently present along with DMNB, it does not serve well as a signature compound since it is ubiquitous in commodities. That leaves only DMNB as a good target compound. Future sampling of aged and untagged Semtex and new Semtex samples should be studied to identify other possible volatile signatures.

For C-4 explosives, cyclohexanone, DMNB, and 2-ethyl-1-hexanol were the 3 most dominant compounds extracted. Cyclohexanone is a solvent that is used in the production of RDX explosive but is primarily used in the production of nylon. 2-ethyl-1-hexanol is primarily used in the manufacture of the diester bis(2-ethylhexyl) phthalate (DEHP) plasticizer, which are present in plastics at up to $40 \%$ by mass. Both cyclohexanone and 2-ethyl-1-hexanol can serve as candidate signature compounds for the detection of C-4 explosive. However, the latter is more likely to produce false positives because plastics are widely used in everyday commodities. Hence, if detection of C-4 can be based on both compounds in the case of untagged explosive and one of the two compounds along with DMNB taggant in the case of tagged explosives, the false 
alarm rate can be reduced. A summary of the compounds detected for each of the explosive cases by GC/MS is presented in Table 23 .

Table 23. Summary of the volatile components detected by SPME-GC/MS(x) \& SPME-IMS at one universal setting (a).

\begin{tabular}{|c|c|c|c|c|c|}
\hline EXPLOSIVES & $\begin{array}{l}\text { Acetic } \\
\text { acid }\end{array}$ & 1-butanol & Toluene & $\begin{array}{l}\text { n-butyl } \\
\text { acetate }\end{array}$ & DMNB \\
\hline Flex X, untagged (Case 1) & $\mathrm{x}$ & $\mathrm{x}$ & $\mathrm{x}$ & $\alpha$ & ---- \\
\hline Flex X, untagged (Case 2) & $\mathrm{x}$ & $\mathrm{x}$ & $\mathrm{x}$ & $a$ & ---- \\
\hline PrimaSheet1000, (Case 3) & $\mathrm{x}$ & $\mathrm{x}$ & $\mathrm{x}$ & ---- & $a$ \\
\hline EXPLOSIVES & Acetone & Undecane & Dodecane & & DMNB \\
\hline Semtex H, tagged (Case 1) & $\mathrm{x}$ & $\mathrm{x}$ & $\mathrm{x}$ & & a \\
\hline Semtex H, tagged (Case 2) & $\mathrm{x}$ & $\mathrm{x}$ & $\mathrm{x}$ & & $\alpha$ \\
\hline Semtex H, tagged (Case 3) & $\mathrm{x}$ & ---- & ---- & & $a$ \\
\hline EXPLOSIVES & \multicolumn{2}{|c|}{ Cyclohexanone } & \multicolumn{2}{|c|}{ 2-E-1-hexanol } & DMNB \\
\hline Composition C-4 & $a$ & & $\mathrm{x}$ & & $a$ \\
\hline
\end{tabular}

\subsubsection{Fiber Chemistry Study by GC/MS}

Positive product ion formation within the IMS analyzer is based on the ability to compete for the reactant ion, which mostly depends on the compound's proton affinity,[17] but the initial mass of the compound available is also critical. Therefore, different SPME fiber chemistries were tested to determine which would provide the best extracted mass ratio for compounds of interest in the detection of the target explosive. The objective was to detect multiple peaks representing multiple target compounds, because the capability to detect multiple signatures would provide a second dimension for detection, thus lowering the false alarm rate.

Figure 57 shows the comparison of 5 different fiber chemistries, CAR/PDMS, PDMS, DVB/CAR/PDMS (StableFlex), PDMS/DVB (StableFlex), and PDMS/DVB by GC/MS for the extraction of the explosives. Figure 57(i) is the result for the extraction of Flex X untagged explosive. The fiber chemistry study was performed on the untagged explosive rather than the 
tagged explosive to ensure that the extraction of n-butyl acetate was optimized because it is readily apparent from Figure 57(ii) and (iii) that DMNB is very well extracted by all of the 5 fiber chemistries. The best chemistry of choice for the extraction of Flex X untagged explosive was determined to be PDMS/DVB since more of n-butyl acetate was detected than acetic acid and 1butanol (which can also be from the breakdown of n-butyl acetate in the GC column). All the fiber coating chemistries other than PDMS provided very high extraction efficiency for n-butyl acetate. For Semtex H explosive shown in Figure 57(ii) both PDMS/DVB and PDMS/DVB (StableFlex) provided better extraction ratios between DMNB and unwanted volatiles compared to the other 3 fiber chemistries. The extraction ratio between cyclohexanone, 2-ethyl-1-hexanol and DMNB from C-4 explosive was almost equivalent for 5 types of fiber chemistry as shown in Figure 57(iii); hence any of the fiber chemistries can be used except for PDMS, which has slightly lower total extraction efficiency. 

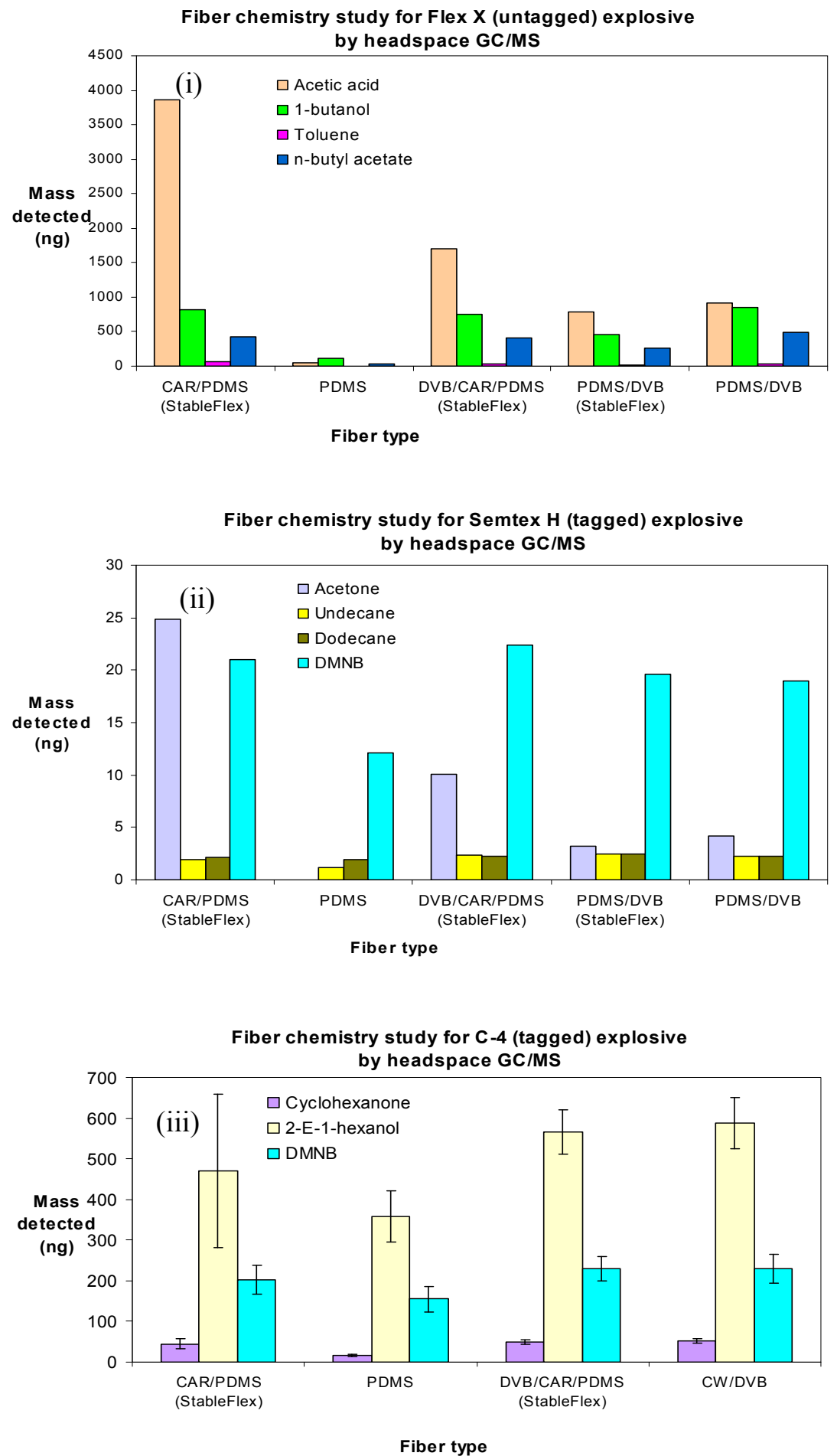

Figure 57. Comparison of different fiber chemistry for headspace extraction of (i) Flex X, 20 min. (ii) Semtex H, 20 min., and (iii) C-4, 5 min by GC/MS. 


\subsection{Headspace SPME-IMS analysis of Detasheet, Semtex H, and C-4}

Vapors from Detasheet, Semtex H, and C-4 explosive samples were successfully detected using IMS by means of headspace air sampling, with detection algorithms based on the volatile chemical signatures associated with the targeted explosives. Figure 59 (i) shows the overlaid spectra of a 20 minute SPME extraction in the headspace of a $15 \mathrm{~mL}$ glass vial containing $1 \mathrm{~g}$ of Flex X explosive and n-butyl acetate standard. Two peaks were presumptively identified as monomer and dimer of n-butyl acetate at the drift time $8.7 \mathrm{msec}$ and $11.4 \mathrm{msec}$, respectively. Multiple peak detection resembling multiple compounds from monomer and dimer formation would provide higher discrimination power for the detection of the target substance. Figure 59 (ii) shows the overlaid spectra of a 20 minute SPME extraction of Semtex H, where DMNB was detected at drift time of $10.2 \mathrm{msec}$. The explosive C-4 was tested at a temperature $30{ }^{\circ} \mathrm{C}$ higher than the universal drift tube temperature determined in this study. The associated DMNB peak was still detectable, but at a shorter drift time, $8.3 \mathrm{msec}$ as shown in Figure 59 (iii). A 5 minute extraction of C-4 explosive yielded a strong peak for DMNB, and a very small peak for cyclohexanone at $7.6 \mathrm{msec}$. Table 23 above also shows the summary of the compounds detected by IMS from each of the explosive cases, and Table 24 below reports the limits of detection for each of the compounds on the GE Itemiser 2 in the positive operating mode, and their reduced mobilities $\left(\mathrm{K}_{0}\right)$ determined using the Smith IonScan 400B. 


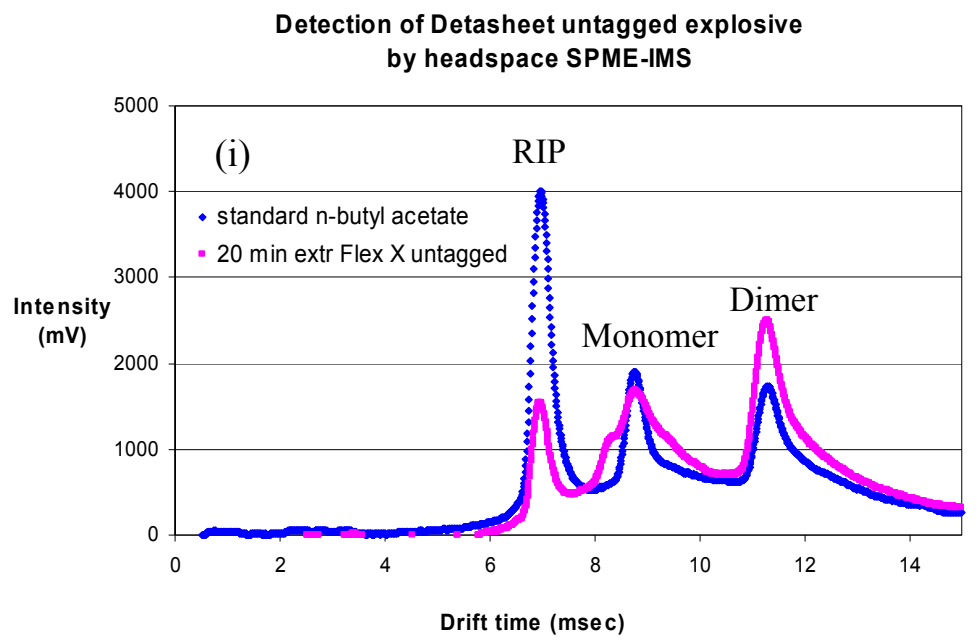

Detection of Semtex H (tagged) explosive by headspace SPME-IMS

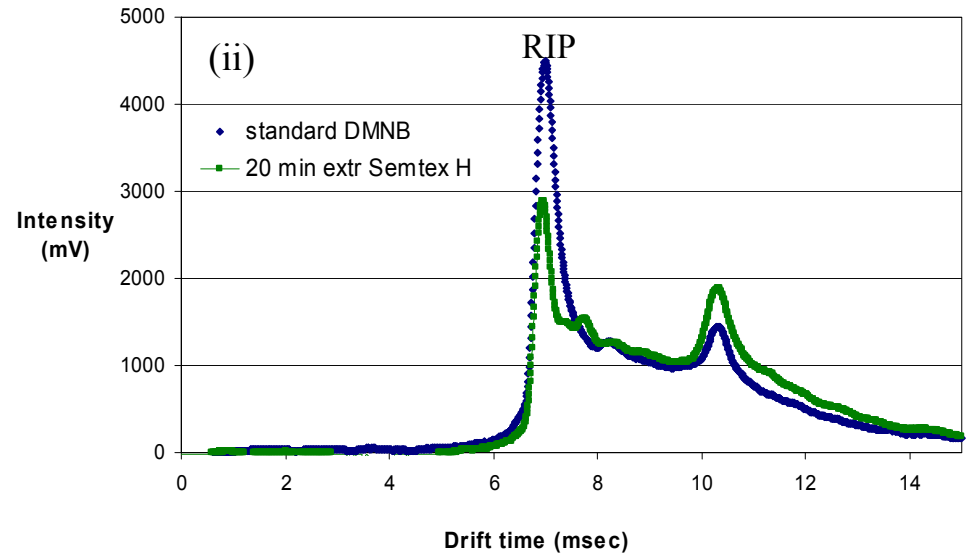

Detection of $\mathrm{C}-4$ (tagged) explosive

by headspace SPME-IMS

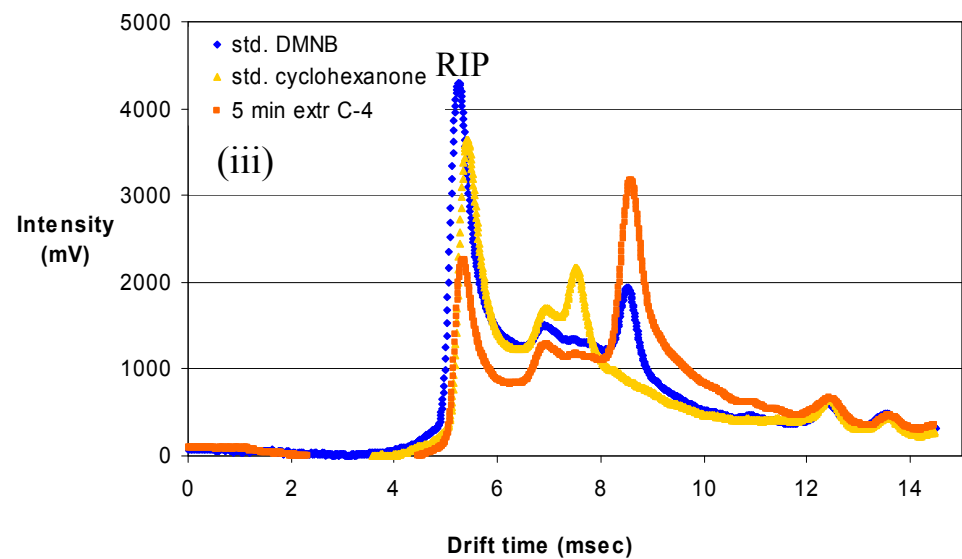

Figure 59. SPME-IMS extraction time curves of (i) Flex X, (ii) Semtex H, and (iii) C-4 at optimized condition. 
Table 24. Reduced mobility values $\left(\mathrm{K}_{0}\right)$ and Limits of Detection by IMS.

\begin{tabular}{|l|c|c|} 
Compounds & $\mathrm{K}_{0}\left(\mathrm{~cm}^{2} \cdot \mathrm{V}^{-1} \mathrm{~s}^{-1}\right)$ & LOD by SPME-IMS \\
\hline DMNB & 1.40 & $1.6 \mathrm{ng}$ \\
\hline Cyclohexanone & $1.85,1.52$ & $2.6 \mathrm{ng}$ \\
\hline n-butyl acetate & 1.72 & $2.2 \mathrm{ng}$ \\
\hline
\end{tabular}

The positive operating mode in IMS is prone to higher false positive alarm rates compared to the negative operating mode because of the ease with which neutral molecules cluster with $\mathrm{H}^{+}\left(\mathrm{H}_{2} \mathrm{O}\right)_{n}$ as compared to $\mathrm{O}_{2}^{-}\left(\mathrm{H}_{2} \mathrm{O}\right)_{n}$ reactant ions.[17] The rate of false positive alarms can be reduced for IMS in positive polarity if the detection algorithms can be based on multiple target peaks. Because the headspace of the two plastic explosives in this study contains compound(s) that produced 2 or more peaks in the IMS, multiple channel detection algorithms can be implemented on Flex X, and C-4,.

\subsubsection{Minimum SPME extraction time (mass) for positive alarm}

The SPME extraction time profiles shown in Figure 59 (i), (ii) and (iii) are for Flex X, Semtex H, and C-4 explosives, respectively. The extraction from the headspace of Flex X, and C-4 explosive quickly reached the equilibrium extraction time after 5 , and 4 minutes, respectively. After this equilibrium time, extended SPME sampling time no longer increased the intensity in the IMS. It was also observed that instantaneous ( $<1 \mathrm{sec}$ of fiber exposure) extraction of Flex X explosive already extracted enough n-butyl acetate to form dimer product ion swarms. On the other hand, the Semtex H extraction took much longer ( $1 \mathrm{hr})$ to achieve SPME extraction time equilibrium. 
SPME IMS extraction time curve for Flex X (untagged) explosive

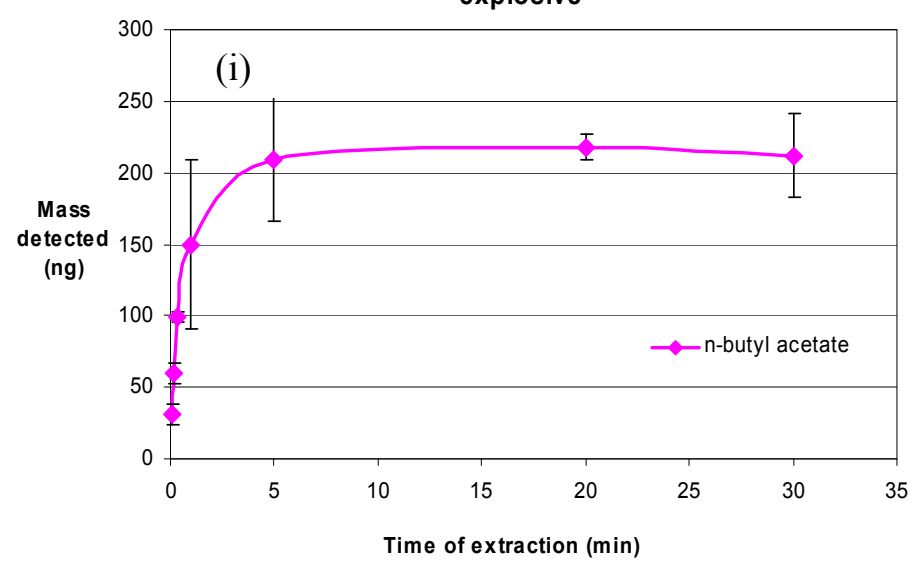

SPME IMS extraction time curve for Semtex H (tagged) explosive

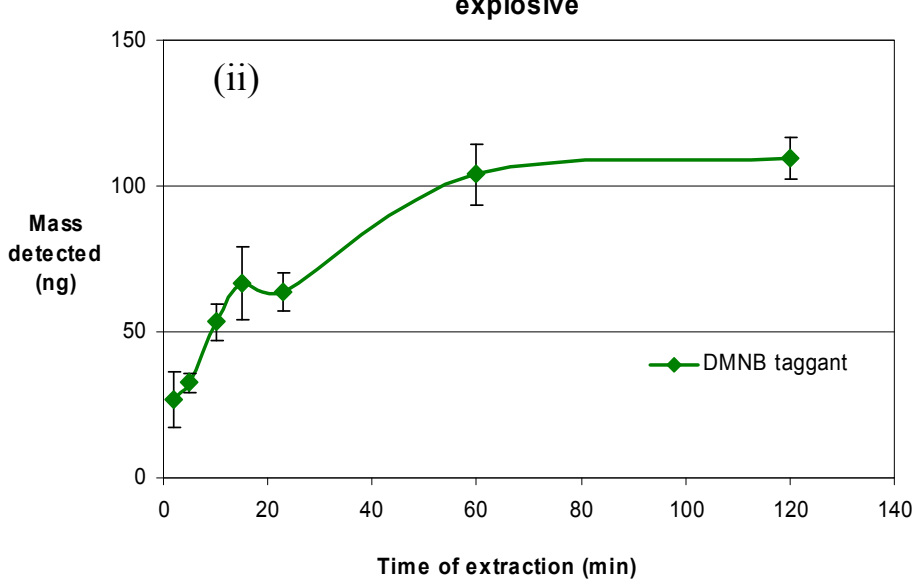

SPME IMS extraction time curve for $\mathrm{C}-4$ (tagged) explosive

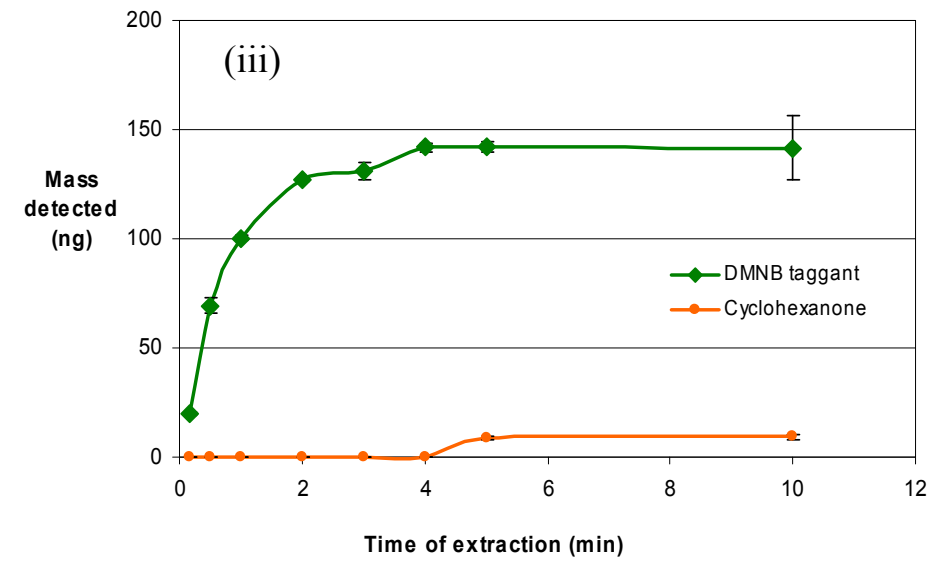

Figure 60. SPME-IMS extraction time curves of (i) Flex X, (ii) Semtex H, and (iii) C-4 at optimized condition. 
The minimum SPME sampling time required for a true positive alarm from a sample containing $1 \mathrm{~g}$ of explosives sealed for 1 week in a $15 \mathrm{~mL}$ glass was also determined, to provide a reference point for future researchers when conducting field sampling of real sample sizes. The headspace of Flex X untagged explosive is saturated with n-butyl acetate. $25 \mathrm{ng}$ of n-butyl acetate was detected with a sampling time of only $5 \mathrm{sec}$, which is 11 times the LOD of the IMS instrument. For C-4 explosive, $20 \mathrm{ng}$ of DMNB was detected with a $10 \mathrm{sec}$ sampling time, which is 12 times the detection limit. Semtex $\mathrm{H}$ explosive required a slightly longer sampling time; 2 minutes were required to detect $25 \mathrm{ng}$ of DMNB. These same explosives were also sealed for a shorter time period of $24 \mathrm{hr}$ instead of 1 week. The peak intensities observed were similar in both cases, which suggest shorter sealing times are possible because the headspace concentrations of the volatile signatures being targeted build up quickly. Air sampling in open environments is expected to be much more challenging because of the large volume and fast diffusion of volatiles in the air. Compared to a sealed environment, the headspace concentrations of the volatiles under such conditions would be much more dilute as, even at positions close to the emitting source. It is expected that longer extraction times than the times reported in this study may be necessary. Improvements in SPME capacity may also be needed to further increase extraction efficiency of the analytes. 


\section{CHAPTER 14: EVALUATION OF SIMION/SDS SIMULATION METHOD OF MODELING IMS}

CONTENT

PAGE

14.1 Drift Gas Flow Rate Effects 173

14.2 Drift Gas Composition Effects 176

14.3 Gate Width Effects 179

To evaluate the predictive power of the SIMION/SDS modeling software, several parameters of the PCP IMS were varied to compare the effects they have on the spectrum drift time and peak shape. The drift and carrier gas flow rates were chosen to demonstrate how drift time, peak shape (i.e., full width at half maximum (FWHM)), and signal intensity change as flow rates were varied. The drift gas composition was varied to illustrate the effect on the resolution between two adjacent peaks. Finally, the ion gate width was varied to illustrate changes in ion intensity and peak broadening. The simulated spectra were compared to the experimental spectra to identify trends in the data because each of the changes caused by varying the operating parameters. In general, it was expected that SIMION/SDS would correctly predict the trends related to the changes rather than the exact values of the experimental outcomes. How well the simulation data coincide with the observed data from the real instrument is dependent on how closely the computational models and methodology mimic the characteristics of the PCP IMS instrument.

\subsection{Drift Gas Flow Rate Effects}

In Simulation 1 the drift:carrier gas flow rates were set to the manufacturer suggested settings of 500:150 $\mathrm{ml} \cdot \mathrm{min}^{-1}$. In Simulation 2 the two flow rates at the inlets were set to equal 
each other $\left(100: 100 \mathrm{ml} \cdot \mathrm{min}^{-1}\right)$; however, because the diameter of the ionization region is less than the diameter of the drift tube, the carrier and drift gas velocities were not equal. When the carrier gas velocity was higher than the drift gas velocity, as in Simulation 2, the carrier gas penetrated through the gate before turning around and exiting the outlet with the drift gas as shown by the flow trajectories in Figure 24. In Simulation 3 the inlet flow rates were set at $\left(0: 150 \mathrm{ml} \bullet \mathrm{min}^{-1}\right)$, where the drift flow was completely shut off. Lastly, in Simulation 4, the flow rates were set at $500: 56 \mathrm{ml} \cdot \mathrm{min}^{-1}$ to achieve equal gas velocities at the point where the two gases met. Three output values; drift time, peak intensity, and FWHM were recorded to assess ability of SIMION/SDS to simulate and detect changes due to variation in the flow conditions.

Figure 61 (i) shows the effects of flow rates on the drift time of TNT in negative operating mode. Detection algorithms for commercial IMS instruments usually allow a variability window of $\sim 50 \mu \mathrm{sec}$ for positive peak detection. Thus, the change reported for TNT drift times from $9.702 \mathrm{msec}$ in Experiment 1 and $9.625 \mathrm{msec}$ for Experiment 3 is significant and reflects faster travel of the ion swarms when the drift gas is completely shut off. Simulation 1 and 3 reported 10.69 and $10.67 \mathrm{msec}$, respectively. While trends for the experimental and simulated drift times were similar, the simulated drift times predicted were within $10 \%$ of the experimental values.

Additionally, both the experimental and the simulated results showed no significant effect of flow rates on the FWHM for TNT, which is in agreement with experimental data reported by Eiceman for benzene, toluene, naphthalene, and anthracene.[107] The peak intensity was highest at $771 \mathrm{mV}$ for Experiment 3 without drift flow as compared to $418 \mathrm{mV}$ when the flow rates were at the default settings in Experiment 1. There are examples in the literature that report the change in product ion intensity when the drift gas [107] or carrier gas [122] flow rates are varied. The 
simulated data, however, did not project such significant differences in peak intensity among the four simulations. The simulations lacked this peak intensity change because the flow conditions in the model allowed ions to pass through the gate at a constant flux. As a consequence of the gate width being held constant for all the four simulation conditions, each simulation allowed approximately the same number of ions to pass into the drift region.
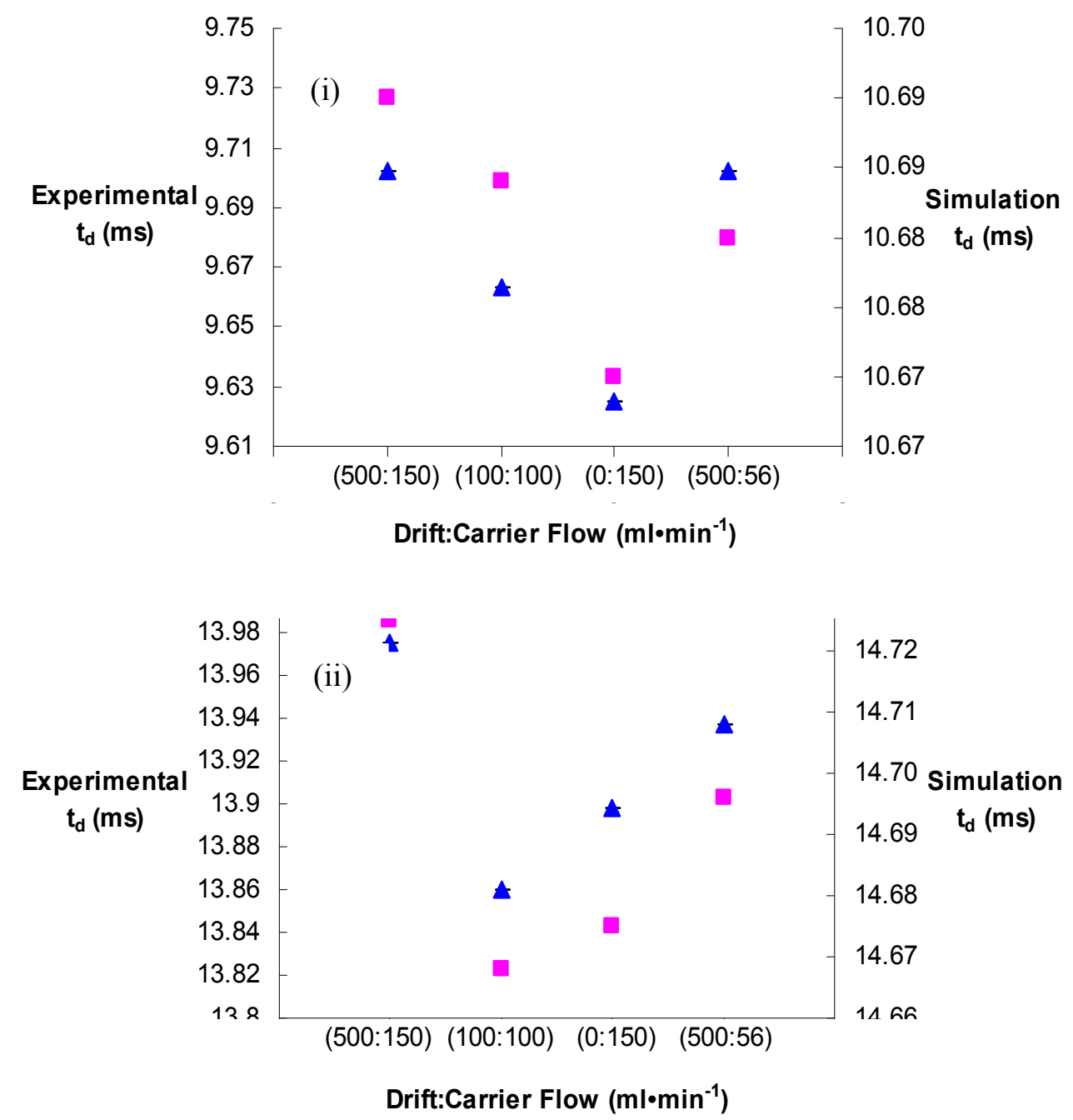

Figure 61. Comparison of experimental and simulated drift times for (i) TNT and (ii) cocaine at various flow-rate conditions. Experimental ( $\boldsymbol{\Delta})$ and simulated ( $\square$ ) data. 
Cocaine was used as the test compound for flow rate experiments and simulations in the positive operating mode. The simulated drift times predicted by the model were within $6 \%$ of the empirical experiments. The trends of ion drift time predicted by the simulations tracked the experimentally determined trends (Figure 61 (ii)). The effect of different flow conditions on cocaine drift time was most noticeable between Experiment $1(13.975 \mathrm{msec})$ and Experiment 2 (13.86 msec). Simulations 1 and 2 reported 14.725 and $14.668 \mathrm{msec}$, respectively. Lastly, the FWHM of cocaine peak was also not particularly affected by the flow rate conditions as observed in the experimental as well as the simulation results. The peak intensity in Experiment 1 was 856 $\mathrm{mV}$ and decreased to $771 \mathrm{mV}$ in Experiment 3. Similar to TNT, varying drift gas flow rates in the simulations did not predict significant changes in peak intensity.

\subsection{Drift Gas Composition Effects}

Both commercial and in-house built IMS instruments usually utilize ambient air as a drift gas. The use of SIMION/SDS in this study to predicted the resolution between two adjacent compounds, TNT and DNF, in air. The study produced excellent correlation between the simulation model and the experimental results (Figure 62). The shoulder peaks seen in Figure 62 (ii) are artifacts of the 'spline fit' function used to plot the simulated spectrum. They could be minimized if more ions could be modeled in the simulation. The experimental and simulated resolutions obtained in air were 1.392 and 1.436, respectively (a difference of $3 \%$ ). This result was achieved despite the simulation and experimental drift times of the individual peaks not matching exactly because of assumptions made related to the instrument dimensions. However, these assumptions should affect all ion species in the same manner, so it is not surprising that the SIMION/SDS simulation software correctly predicted the resolution within the PCP IMS instrument when air is used as the drift gas. The capability to predict the resolution of an IMS 
instrument design is a powerful tool for designing future instruments because it can reduce the costs of the trial-and-error approach.
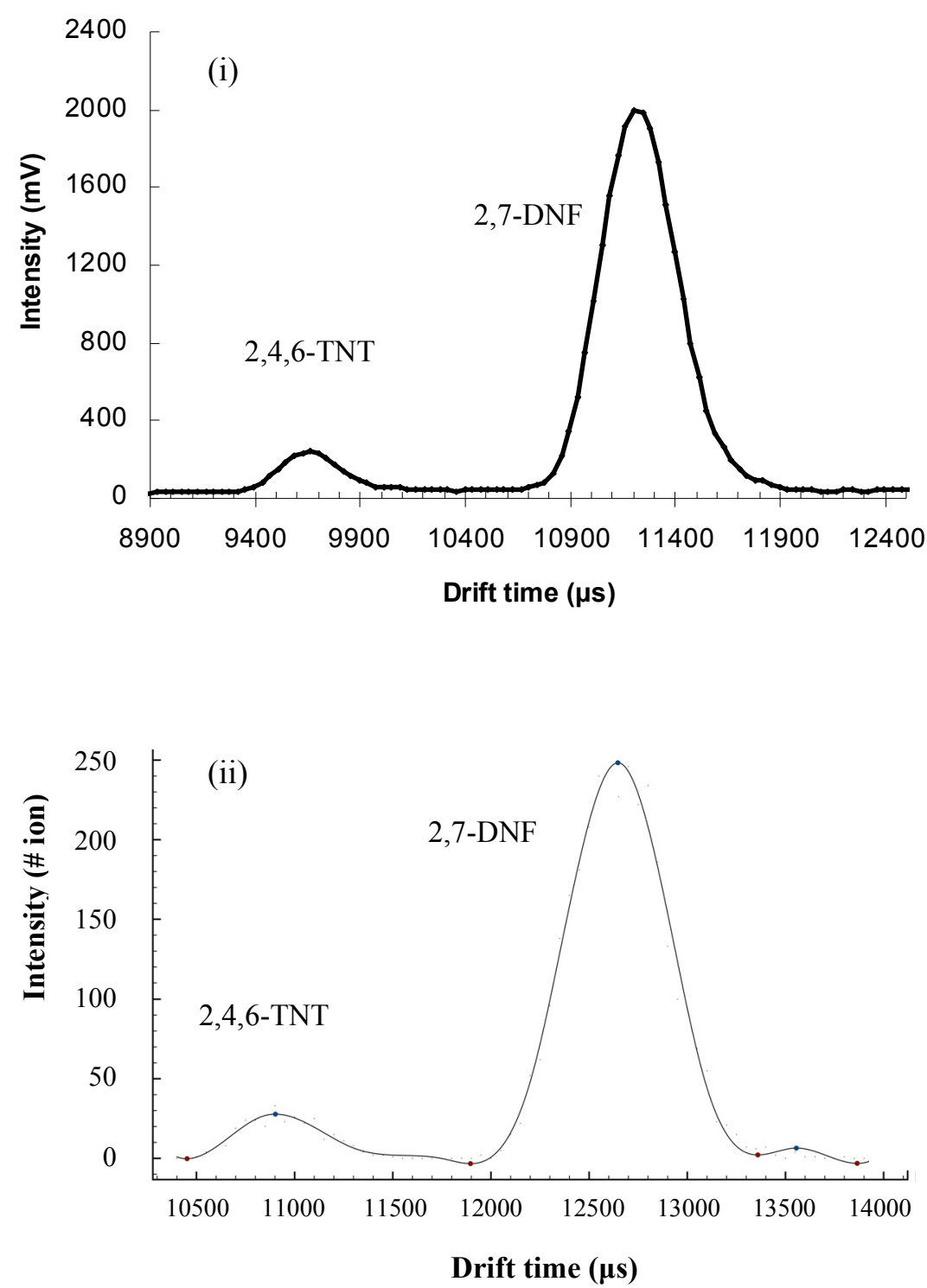

Figure 62. (i) Experimental and (ii) simulated spectra of 2,4,6-TNT and 2,7-DNF with air as both carrier and drift gas.

Since $\mathrm{K}$ and the drift time reflect the collision cross section and mass of the buffer gas, as noted in earlier in the IMS theory section, it is possible to resolve two peaks by changing the drift 
gas because the effect of the collision gas on drift time of the two ion species may be different.[108] Citations in the literature report that, in general, ion travel faster in drift gases that are less massive and less polarizable.[17, 108] The polarizability of helium gas is very low $(0.205$ x $\left.10^{-24} \mathrm{~cm}^{3}\right)$ when compared to argon $\left(1.641 \times 10^{-24} \mathrm{~cm}^{3}\right)$, and He is also less massive.[17, 108] It was expected and observed experimentally that the resolution between two peaks would be lower when using helium and higher when using argon as the drift gas. Shown in Figure 63, when the drift gas was changed from air to helium, the experimental resolution for TNT and DNF decreased to 1.296 from 1.392. When the drift gas was changed to argon, the experimental resolution increased to 1.501 .

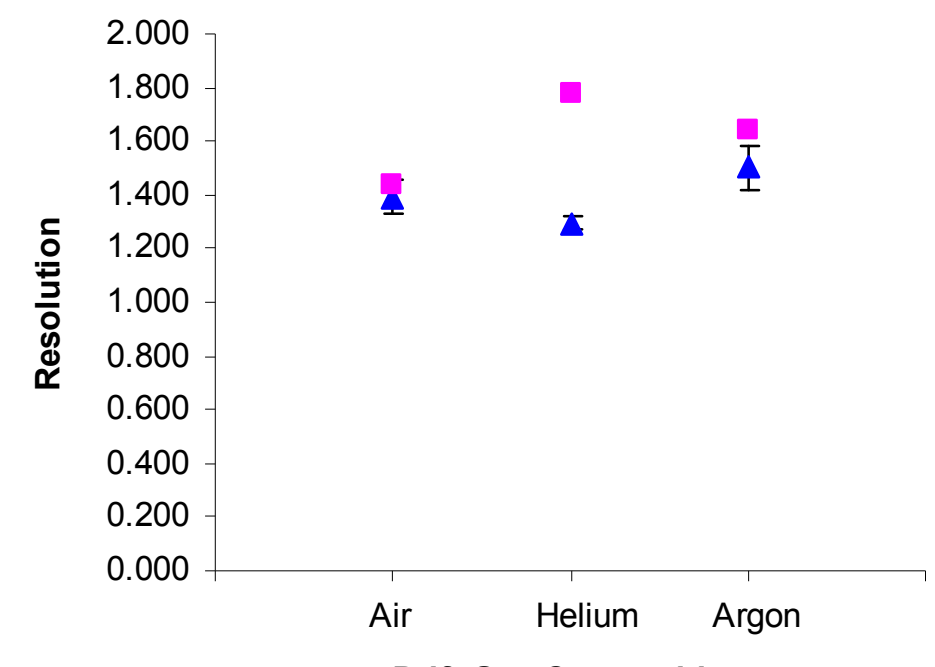

Figure 63. Comparison of experimental versus simulated resolution between 2,4,6-TNT and 2,7DNF in different drift gases. Experimental $(\boldsymbol{\Delta})$ and simulated ( $\square$ ) data.

In a study by Asbury and Hill, improvement in resolution between chloroaniline and iodoaniline was experimentally observed when argon was used as drift gas.[108] However, another study by Hill's research group reported the opposite trend for two other compounds, lorazepam and diazepam, where helium drift gas provided better resolution.[145] While the 
simulations predicted resolution in air within $3 \%$ accuracy, the simulated resolution trends and values for helium and argon mediums were not as accurate. As shown in Figure 63, the simulations predicted an increasing trend from air to helium, instead of decreasing trend as observed in the experimental data.

For argon, the simulation predicted a much higher resolution than was observed experimentally. The lack of agreement between the simulation and experimental values in helium and argon drift gas is primarily due to a limitation of SDS, as it currently cannot accommodate two different gases introduced at different locations. Therefore, the helium and argon simulations had to use the same gas (He or Ar) for both the carrier and drift gas, which is not a perfect match for the experimental conditions. Attempts to experimentally change the carrier gas from air to $\mathrm{He}$ or Ar were made, but as expected, no ion swarms were produced because the PCP IMS uses a

${ }^{63} \mathrm{Ni}$ source that requires air or nitrogen to assist the ionization process. In addition, the current SDS user program calculates the ion gas molecule interactions based on a hard sphere model, which has been shown in a previous study by Jarrold and co-workers to be less accurate, especially for gases other than air.[146] Methods to use more advanced features of SIMION to work around this SDS limitation should be addressed in future work.

\subsection{Gate Width Effects}

The ion shutter or gate employed in most commercial IMS instruments is made of coplanar arrays of closely spaced, parallel thin wires.[17, 94, 132] The voltages on alternating wires are changed to open the gate and allow a pulse of ions to enter the drift region. Typically, ions from the reaction region are pulsed into the drift region for only $300 \mu$ sec every 20 to 30 msec; thus, only $\sim 1 \%$ of all ions are available for measurement.[17] The ion gate width is an 
important variable in establishing peak shape and intensity in the mobility spectrum. $[94,123$, 127] The minimum pulse that is practical is between 10 to $100 \mu \mathrm{sec}$, which is determined by the minimum time required for the ion swarms to move through the wire structure from the reaction region side of the gate into the drift region side.[17]

Almost all commercial IMS instruments have a duty cycle not exceeding 1\%.[17] Other ion shutter designs such as the sinusoidal waveform were developed to improve the duty cycle of the gate for better sensitivity.[92] The capability of SIMION/SDS to simulate the ion behavior passing through the gate would give important insights to the development of new gating mechanisms. This study performed a simple evaluation of the SIMION/SDS simulation software by observing the peak intensity and peak broadening as the gate width varied from 50, 100, 250, and $500 \mu \mathrm{sec}$. Peak broadening is the result of several mechanisms that occur within IMS instruments: gate width, diffusion, charge repulsion, ion-molecule reaction, and inhomogeneity in the electric field. $[94,105,123]$ The two first mechanisms, gate width and diffusion, are the major factors determining the shape and the width of the IMS peaks.[105, 108] There are also previous reports in the literature that confirm that an increase in the gate width should result in increased peak intensities and peak widths.[94, 123] 

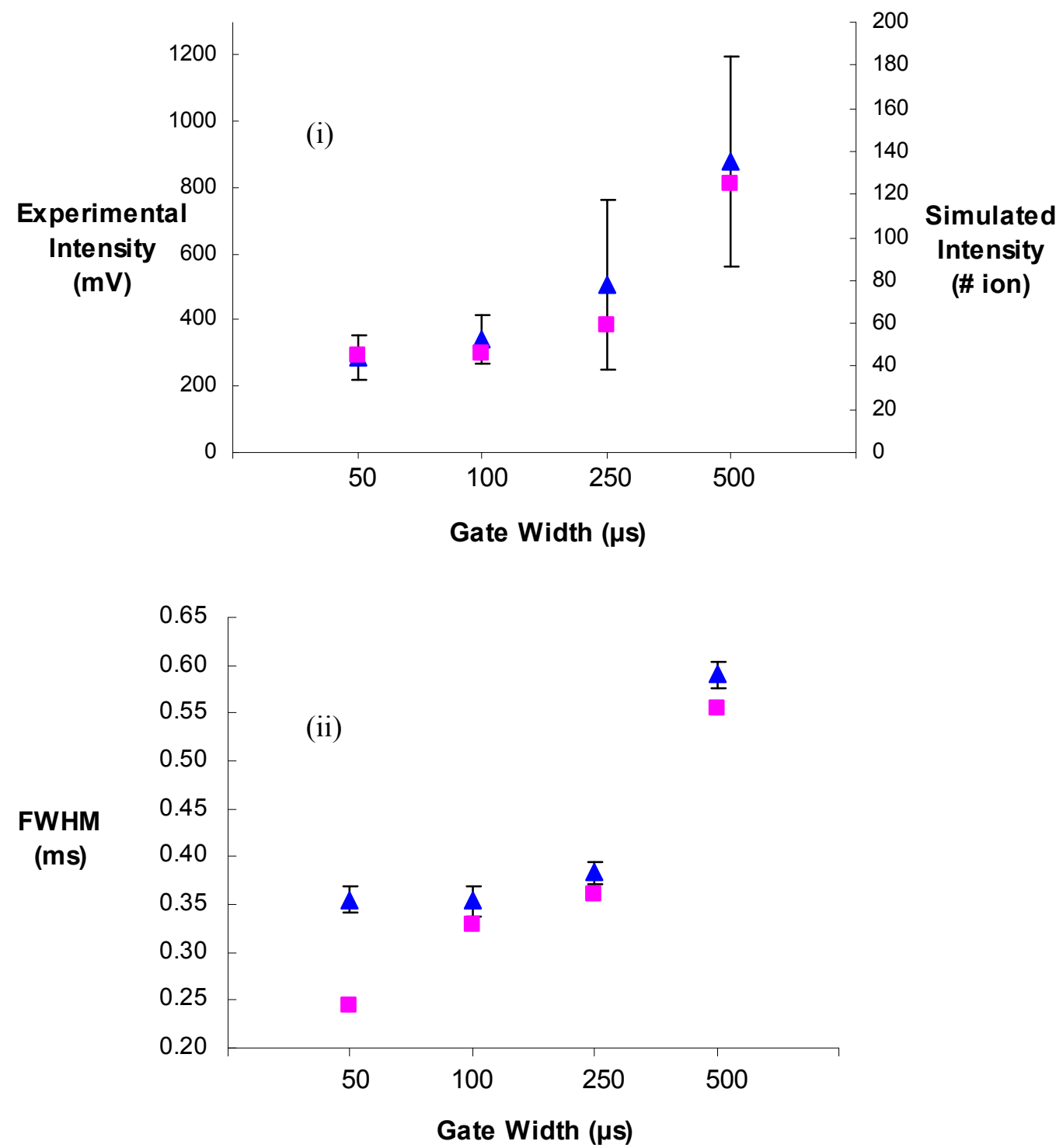

Figure 64. Comparison of experimental versus simulated (i) peak intensity, and (ii) FWHM at various gate width timings. Experimental $(\boldsymbol{\Delta})$ and simulated $(\boldsymbol{\square})$ data.

The results for peak intensity, shown in Figure 64 (i), and for FWHM, shown in Figure 64 (ii), illustrate these expectations. Figure 64 (ii) shows averages of 3 replicates, and the difference between the experimental and simulated full width at half maximum was $\sim 6 \%$. The FWHM predicted were within 6-7\% of the experimental FWHM values, except for Experiment 
8. The experimental FWHMs did not vary significantly when the gate width decreased from 100 $\mu \mathrm{sec}$ to $50 \mu \mathrm{sec}$, while the simulation predicted a decrease in FWHM. This difference may be due to the inability to accurately control the gate width down to $50 \mu \mathrm{sec}$ (which may be true for an instrument that was built in the 80 's), whereas virtual instruments will predict the ideal case. The high error bars in the experimental intensities at gate widths of 250 and $500 \mu \mathrm{sec}$ may be the result of inconsistencies in sample introduction within the PCP instrument. In Experiment 8, when the gate was opened for $50 \mu \mathrm{sec}$, not many ions passed through the gate resulting in a peak intensity of only $284 \mathrm{mV}$ and a FWHM of $355 \mu \mathrm{sec}$. At a gate width of $500 \mu \mathrm{sec}$, the peak intensity increased to $878 \mathrm{mV}$ and the peak shape was much broader (FWHM $=590 \mu \mathrm{s})$ as compared to the other three gate settings. As shown in Figure 64 (i), and (ii), SIMION/SDS generally predicted the trends for both the peak intensity and peak broadening (FWHM). The trends observed are also in agreement with the experimental trends reported by Spangler et. al.[123] and Eiceman et. al.[94]

In addition, a visual observation was made when the simulated ion swarms were flying through the gate as the voltage on the wires switched from 'open' to 'closed'. The changes in voltage on the alternating wires caused some ion swarms that just recently passed through the gate to slow down before they re-accelerate and continued down the drift tube. Because ions in viscous environments follow the electric field gradients, changes of electric field gradients can easily alter ion trajectories, especially around wires or grids where electric fields associated with electrostatic refraction can be complicated.[121] However, under the conditions of these experiments, this effect was minor and did not cause significant peak-tailing effects in either the experimental or simulated data. 


\section{CHAPTER 15: DETECTION FIGURES OF MERIT FOR IN-HOUSE FABRICATED IMS}

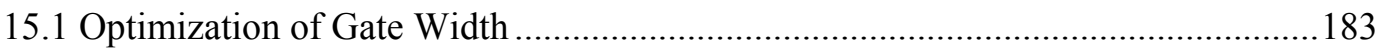

15.2 Optimization of Electric Field at Different Region of IMS....................................184

15.3 IMS Responses of Common Explosives and Illicit Drugs ….................................189

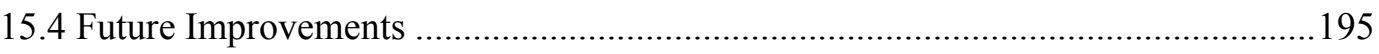

\subsection{Optimization of Gate Width}

It is well understood that an increase in the gate width improves signal intensity because more ions are allowed to enter the drift region. However, a wider initial pulse and the increased space charge effect as the result of more ions translates into a deterioration of the peak resolving power. Figure 65 shows the results obtained for gate width varied between 100 to $1000 \mu$ sec on the FIU IMS at a 10 gain $\left(\mathrm{G}^{\wedge} 10\right)$. The peak intensity and the FWHM varied almost linearly as the gate width increased.

The current BN gate installed on the FIU IMS is not yet an optimal design, therefore a gate width of lower than $100 \mu \mathrm{sec}$ does not allow enough ions to enter the drift region. The analysis of explosives and drug samples using the FIU IMS were conducted using a $250 \mu \mathrm{sec}$ gate width and a 9 gain $\left(\mathrm{G}^{\wedge} 9\right)$. Note that gate width is commonly reduced to improve peak resolving power with a tradeoff in lower signal intensity. Commercial IMS instruments with optimal gate design and electrical shielding can afford to operate at higher gain and, hence, gate widths smaller than $100 \mu$ sec are sufficient for good signal to noise ratio. 

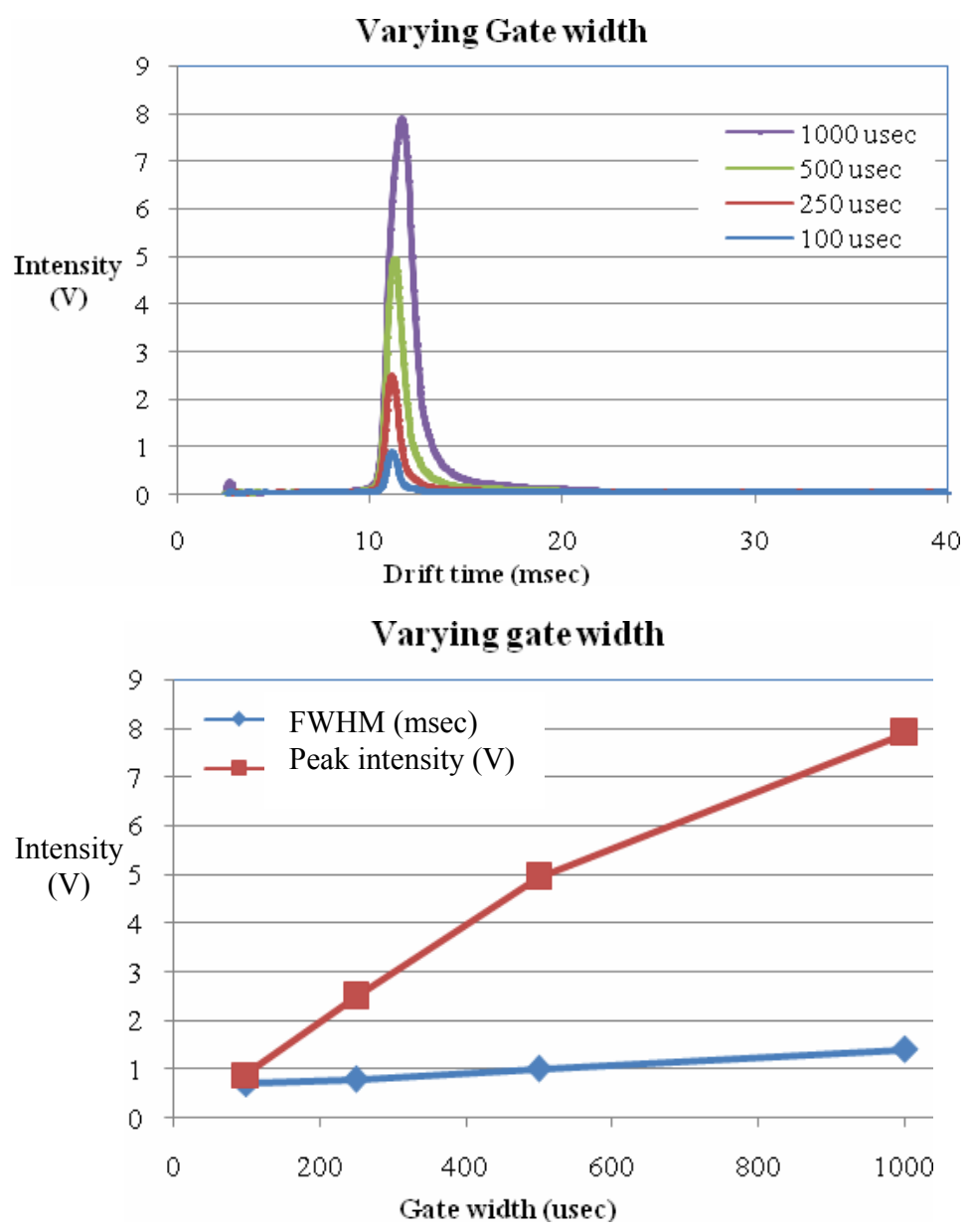

Figure 65. Response of RIP at different gate width for the FIU IMS.

\subsection{Optimization of Electric Field at Different Region of IMS}

The IMS electric field reported in most of the literature is the drift electric field which is the field supplied at the drift region between the gate and the aperture grid ( $\sim 3 \mathrm{~mm}$ from the detector). Very few studies reported the optimization of their IMS electric field at other regions such as between the source and the gate, and between the aperture grid and the detector. In this work, the electric field at all 3 regions of the newly built FIU IMS drift tube were varied to show its effect on the peak intensity and FWHM of the negative reactant ion, $\mathrm{O}_{2}{ }^{-}\left(\mathrm{H}_{2} \mathrm{O}\right)_{n}$. 


\subsubsection{E-field at region between ion source and ion gate}

Optimization of the electric field between the source and the gate region is critical because ions produced in the cylindrical shell need to be transported to the gate region successfully with minimal loss to the wall. Depending on the location of the source, the overall electric field should not be smaller than the field in the drift region. Figure 66 shows the effects of E-field $(\mathrm{s-g})$ on RIP shape and intensity as it was varied from 170 to $320 \mathrm{~V} / \mathrm{cm}$, at a constant gate width $(250 \mu \mathrm{sec})$. The peak intensity (at $\left.\mathrm{G}^{\wedge} 10\right)$ increases linearly and levels out when the $\mathrm{E}$ field $_{(\mathrm{s}-\mathrm{g})}$ reaches $290 \mathrm{~V} / \mathrm{cm}$ (with respect to $235 \mathrm{~V} / \mathrm{cm}$ drift field). The FWHM at all E-field $\mathrm{s}_{(\mathrm{s}) \mathrm{g}}$ settings did not vary significantly $(0.9 \mathrm{msec})$ because the gate width was held constant.
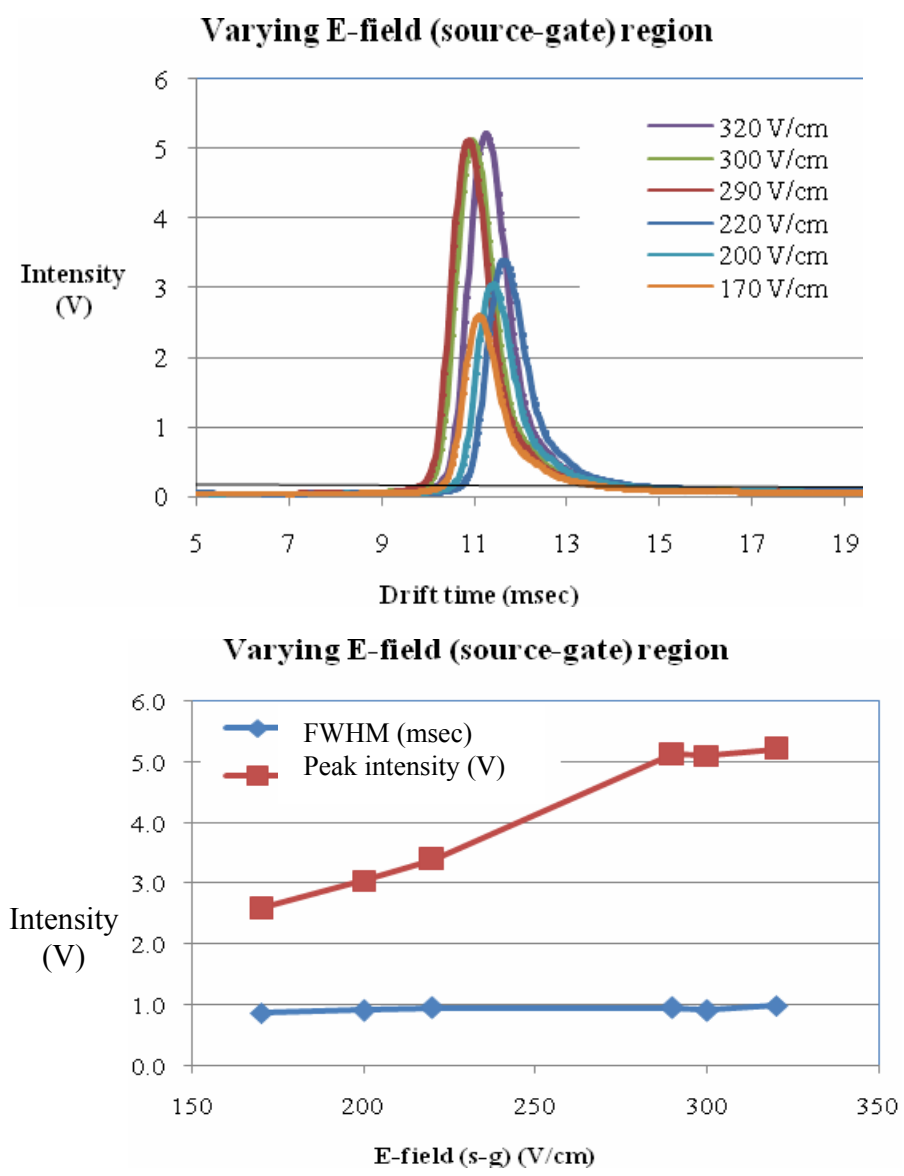

Figure 66. Effects of electric field at source-gate region on the reactant ion's peak response and peak shape. 


\subsubsection{Drift E-field at region between gate and aperture grid}

The drift region is the longest region of the drift tube, where ions of different species are separated. Longer drift times allow ion swarms to diffuse, and some portion of the total ion population is lost to the tube wall as they travel toward the detector. Drift tubes with higher drift electric fields move ions faster and hence leave less time for ion diffusion to take place. This normally results in higher signal intensity and smaller peak width as shown in

Figure 67. When the drift electric field increased from $150 \mathrm{~V} / \mathrm{cm}$ to $235 \mathrm{~V} / \mathrm{cm}$, the ions' drift time decreased from $17.16 \mathrm{msec}$ to $11.28 \mathrm{msec}$. Consequently, the ion intensity improved $\sim 5$ fold, and the FWHM improved by $30 \%$. Depending on the power supply specifications and electrical insulation between drift tube components, drift electric field in some commercial instruments can go as high as $700 \mathrm{~V} / \mathrm{cm}$ to achieve the best possible signal intensity and peak shape. Varying the drift electric field necessitates also optimizing the offset voltage between the alternate gate wires to effectively close the gate. If these voltages are not optimized, the strong drift electric field tends to pull ions past the gate causing a lift in baseline during the gate close cycle. This phenomenon was observed in the FIU IMS when the drift electric field was raised to higher than $400 \mathrm{~V} / \mathrm{cm}$ while the gate field was at $\sim 500 \mathrm{~V} / \mathrm{cm}\left(100 \mathrm{~V}\right.$ offset between $\mathrm{W}_{1}$ and $\mathrm{W}_{2}$ at $2 \mathrm{~mm}$ apart center to center). 
Varying E-field of Drift Region

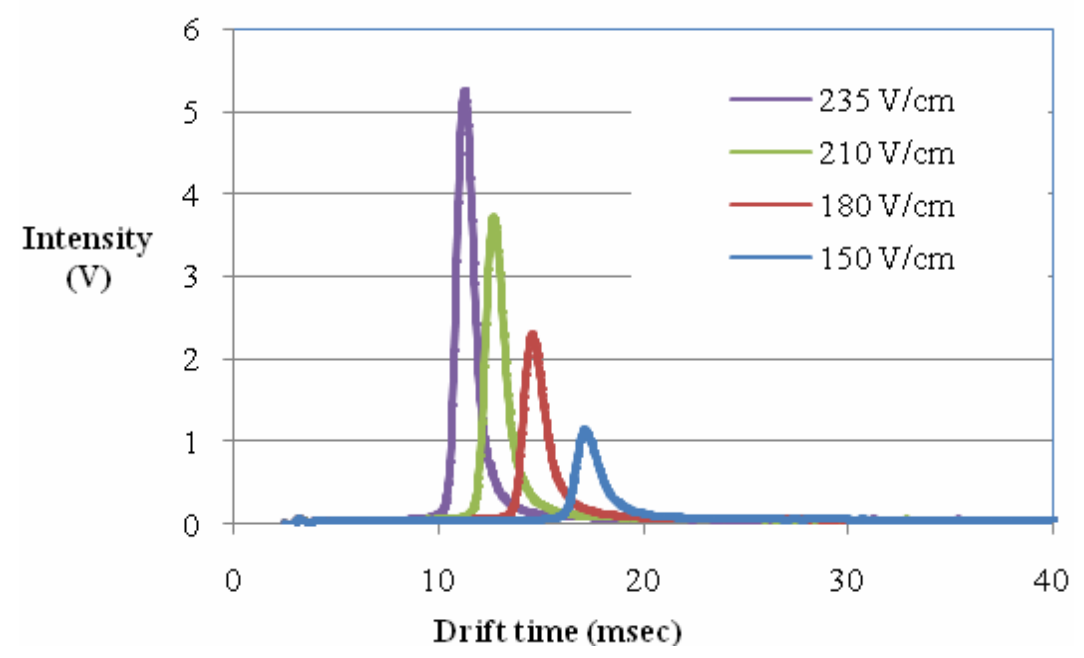

Varying E-field of Drift Region

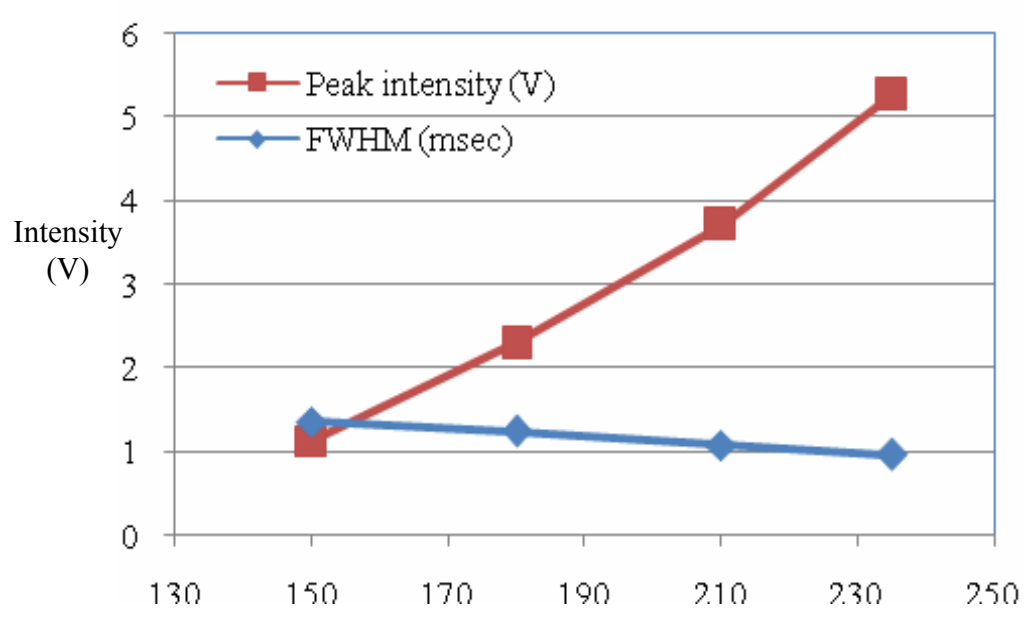


Figure 67. Effects of drift electric field on the reactant ion's peak response and peak shape.

\subsubsection{E-field at region between aperture grid and detector}

As mentioned in chapter 7, the region between the aperture grid and the detector is extremely short $(2-3 \mathrm{~mm})$ but the electrical field in this region is essential to shield the approaching ion swarm from the detector. Insufficient E-field ${ }_{(\mathrm{a}-\mathrm{d})}$ would result in ions lost to the aperture grid and poor peak shape. This effect was observed in the FIU IMS as the E-field $(\mathrm{a-d})$ varied from $200 \mathrm{~V} / \mathrm{cm}$ to $1000 \mathrm{~V} / \mathrm{cm}$. As seen in Figure 68 the peak intensity increases significantly and starts to level out after $820 \mathrm{~V} / \mathrm{cm}$ (for drift electric field fixed at $235 \mathrm{~V} / \mathrm{cm}$ ). As

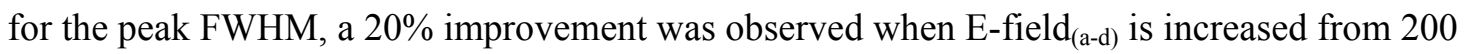
$\mathrm{V} / \mathrm{cm}$ to $600 \mathrm{~V} / \mathrm{cm}$. Thereafter, further increase in field strength does not improve peak shape.
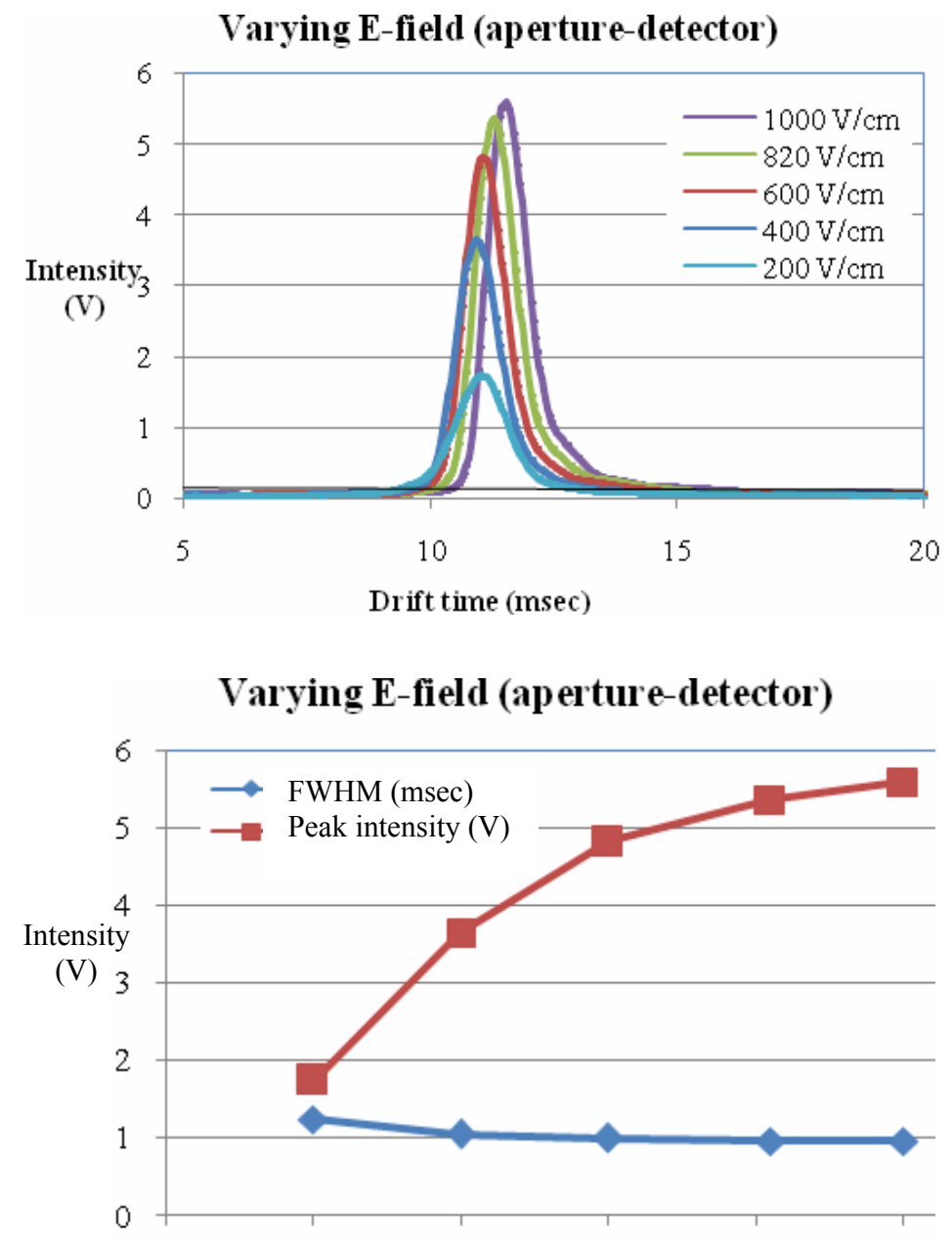
Figure 68. Effects of electric field at aperture-detector region on the reactant ion's peak response and peak shape. 


\subsection{FIU IMS Responses of Common Explosives and Illicit Drugs}

Figure 69 shows the IMS response of some common explosives and their corresponding response curve in negative operating mode, with $\mathrm{CH}_{2} \mathrm{Cl}_{2}$ dopant, at $180{ }^{\circ} \mathrm{C}$, sample flow and drift flow rate at 300 and $500 \mathrm{~mL} \cdot \mathrm{min}^{-1}$, respectively. Product ions of DNT, TNT and RDX were formed and detected at $6.03,6.51$, and $6.78 \mathrm{msec}$, respectively, with the chloride reactant ion $\left(\mathrm{Cl}^{-}\right)$ detected at $3.66 \mathrm{msec}$. Figure 70 shows the IMS response of some common drugs and their corresponding response curve in positive operating mode, with $\mathrm{NH}_{3}$ dopant and at similar drift tube conditions as the explosive analyses above. Product ions of MDA, MA, and Benzoylecgonine were formed and detected at $8.76,8.22$, and $10.95 \mathrm{msec}$, respectively with the reactant ion $\left(\mathrm{NH}^{+}\right)$detected at $5.4 \mathrm{msec}$. 
DNT response

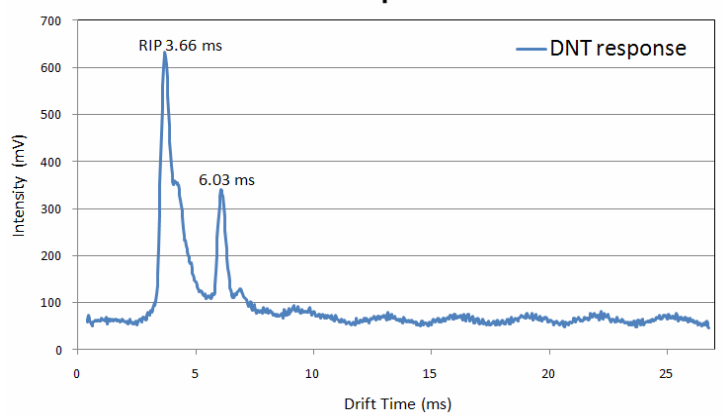

TNT response

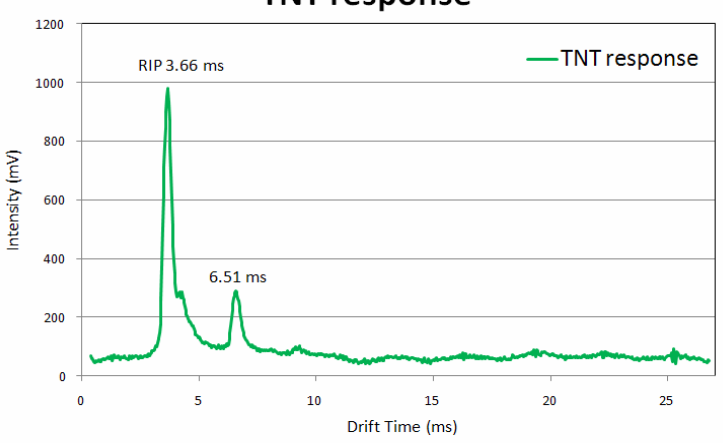

RDX response

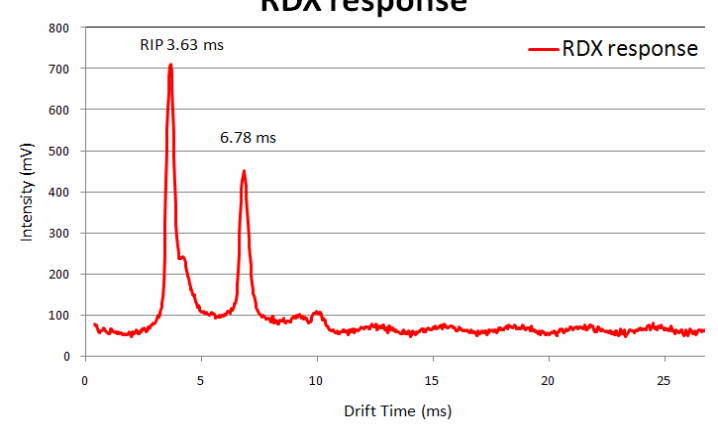

DNT Response Curve

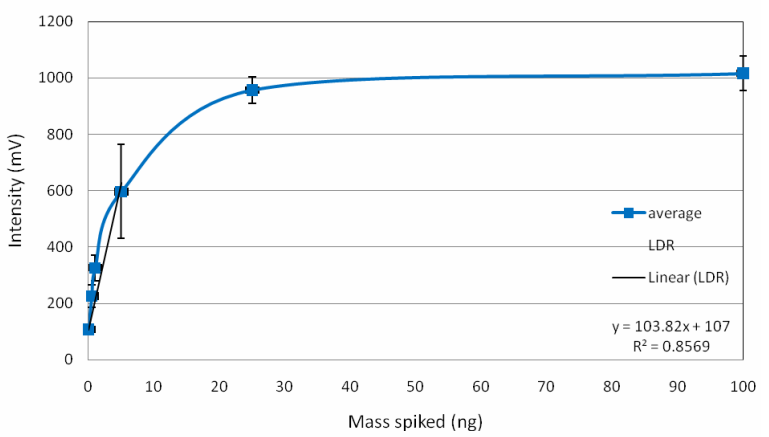

TNT Response Curve

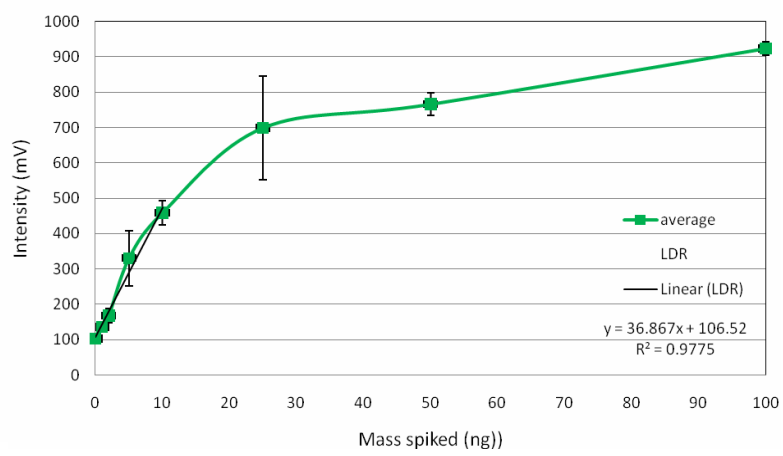

RDX Response Curve

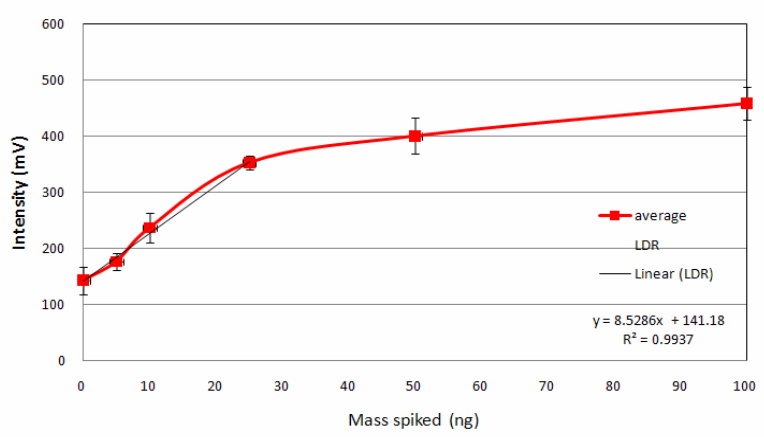

Figure 69. (Left) IMS response of a $1 \mu \mathrm{L}$ spike of 2 ppm 2,4-DNT, 5 ppm 2,4,6-TNT, and 100 ppm RDX in MeOH solution. (Right) IMS response curve of 2,4-DNT, 2,4,6-TNT, and RDX. 

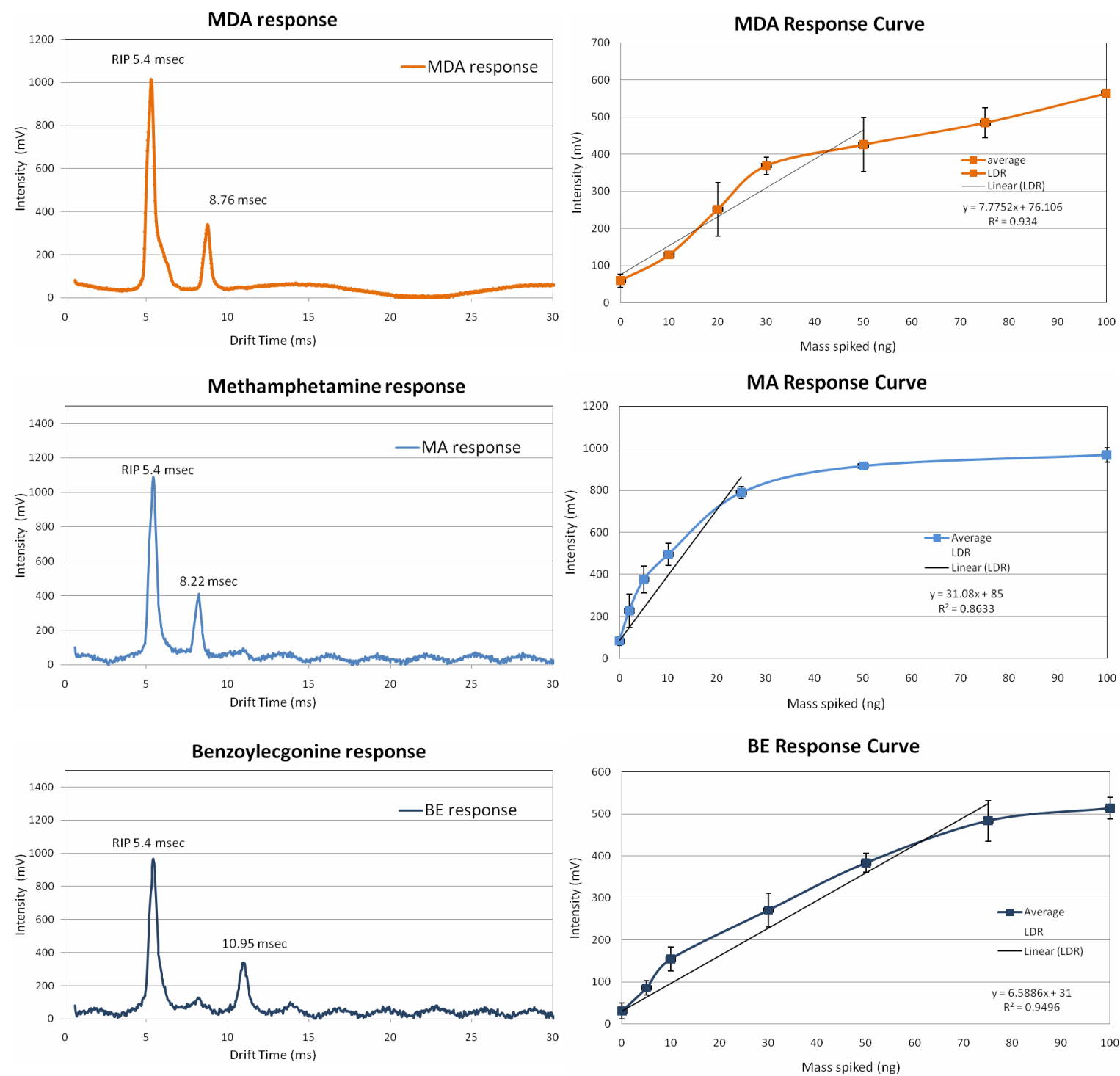

Figure 70. (Left) IMS response from a $1 \mu \mathrm{L}$ spike of $30 \mathrm{ppm}$ MDA, $5 \mathrm{ppm} \mathrm{MA}$, and $30 \mathrm{ppm}$ BE in $\mathrm{MeOH}$ solution, (Right) IMS response curve of MDA, MA, and BE.

The $\mathrm{K}_{0}$ values for each of the product ions from explosives were calculated using PETN as the calibrant compound, which was detected at $8.28 \mathrm{msec}$ (literature $\mathrm{K}_{0}$ of $1.35 \mathrm{~cm}^{2} \cdot \mathrm{V}^{-1} \mathrm{~s}^{-1}$, $\mathrm{CH}_{2} \mathrm{Cl}_{2}$ dopant) shown in Figure 71. For the drug product ions, MDMA was used as the calibrant, 
which has a $\mathrm{K}_{0}$ value of $1.36 \mathrm{~cm}^{2} \cdot \mathrm{V}^{-1} \mathrm{~s}^{-1}$ in ammonia dopant, was detected at $8.91 \mathrm{msec}$ shown in Figure 72.

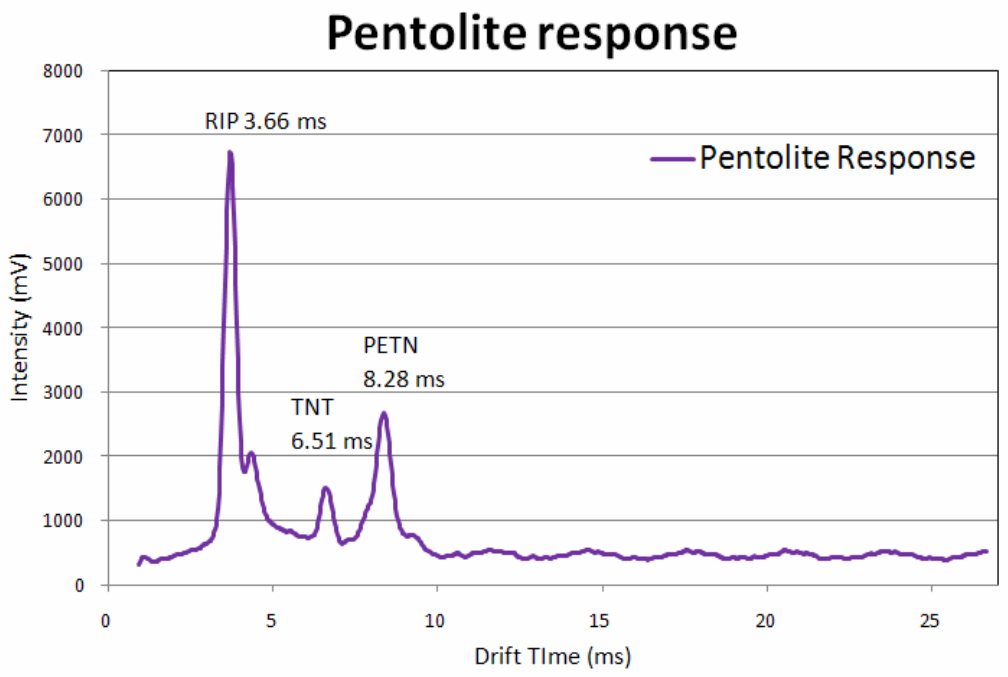

GE IMS Response of Pentolite

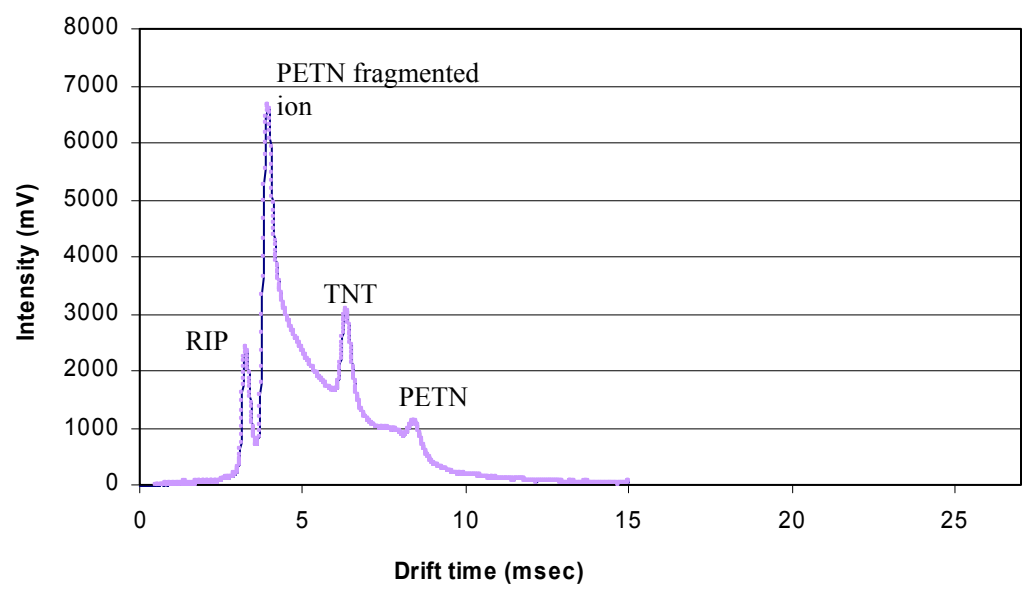

Figure 71. (Top) FIU IMS response (at $\mathrm{G}^{\wedge} 10$ ), and (Bottom) GE IMS response of a $1 \mu \mathrm{L}$ spike of 5 ppm Pentolite explosive in ACN solution. 


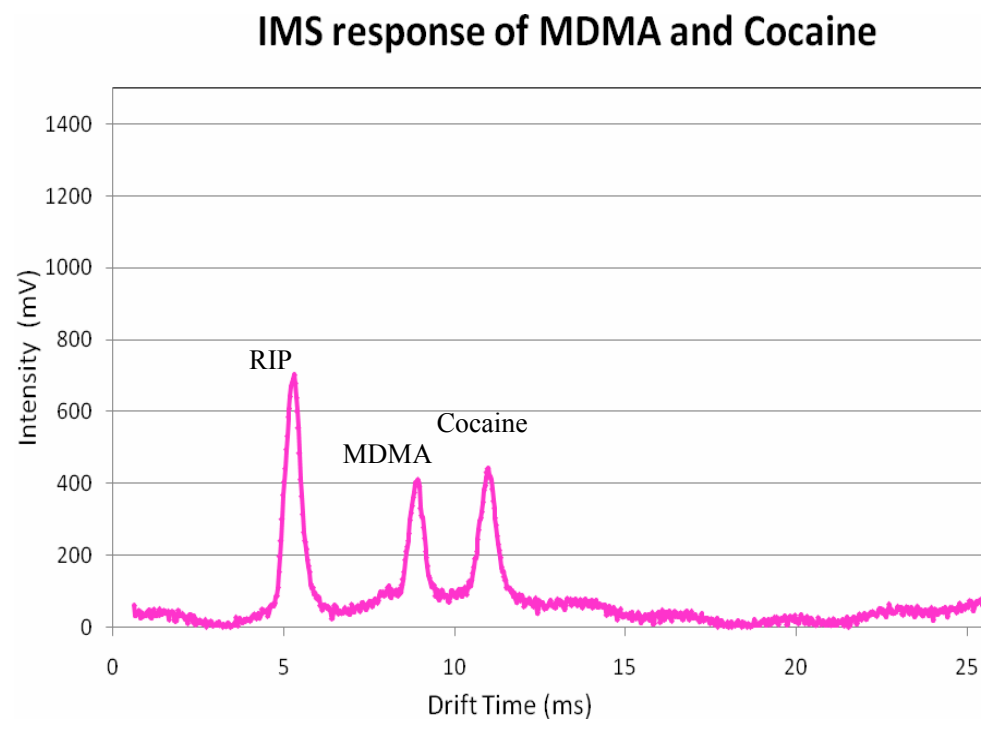

GE IMS Response of MDMA \& Cocaine

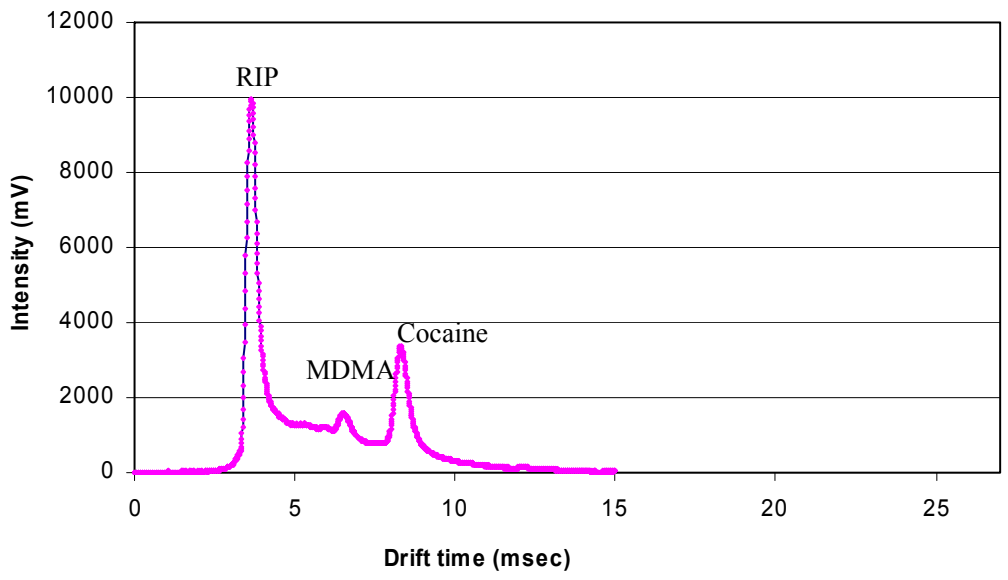

Figure 72. (Left) FIU IMS (at $\mathrm{G}^{\wedge} 9$ ) response of a $1 \mu \mathrm{L}$ spike of $10 \mathrm{ppm}$ MDMA and Cocaine in $\mathrm{MeOH}$ solution. (Right) GE IMS response of a $1 \mu \mathrm{L}$ spike of $1 \mathrm{ppm}$ MDMA and $10 \mathrm{ppm}$ Cocaine in $\mathrm{MeOH}$ solution.

The $\mathrm{K}_{0}$ values calculated for the explosives and drugs analyzed are reported in Table 25, as are the IMS performance measurements such as resolving power $\left(\mathrm{R}_{\mathrm{p}}\right)$, resolution $\left(\mathrm{R}_{\mathrm{pp}}\right)$, limit of detection (LOD), and linear dynamic range (LDR) determined for the FIU IMS. Resolving power 
of the product ion peak formed from each of the explosive and drug was also calculated. As seen in the table, the resolving power for the explosives and drugs obtained from the FIU IMS on average is $\sim 15.3$ and 11 , respectively, very close to the values obtained from a commercial IMS GE Itemizer 2 (average is 14 and 10, respectively). The resolution between TNT and PETN (Figure 71) on the FIU IMS was calculated to be 2.7 and the resolution achieved by the GE IMS was 3.8. In the positive mode, the resolution of MDMA and cocaine (Figure 72) on the FIU IMS was comparable to the GE IMS, (3.0 and 3.1, respectively). The peak to peak resolutions calculated for each of the explosives and drugs with respect to the reactant ion peak (RIP) on the FIU IMS range between 4 and 8 . The limit of detection determined from the above explosive response curves (in Figure 69) for DNT, TNT, and RDX are $1.2 \mathrm{ng}, 0.7 \mathrm{ng}$, and $2.5 \mathrm{ng}$ respectively, and for MDA, MA, and Benzoylecgonine are $5 \mathrm{ng}, 2 \mathrm{ng}$, and $8 \mathrm{ng}$, respectively. In general, it is expected that IMS instruments have a picogram to low nanogram detection limit and the FIU IMS instrument sits well within this range. The linear dynamic range however is lower than desired ( $\sim 1$ to $30 \mathrm{ng}$ for most compounds except Benzoylecgonine). Further optimization of the IMS instrument is necessary for improved LDR performance. Different areas of improvement are discussed in the following section (15.4).

Table 25. FIU-IMS performance summary of the analysis of explosives ( $180{ }^{\circ} \mathrm{C} \mathrm{CH}_{2} \mathrm{Cl}_{2}$ dopant), and illicit drugs $\left(180{ }^{\circ} \mathrm{C} \mathrm{NH}\right.$ dopant).

\begin{tabular}{|c|c|c|c|c|c|c|c|}
\hline Explosives & $\begin{array}{c}\text { Drift time } \\
\text { (msec) }\end{array}$ & $\begin{array}{c}\mathbf{K}_{0} \\
\left(\mathrm{~cm}^{2} \cdot \mathrm{V}^{-1} \mathrm{~s}^{-1}\right)\end{array}$ & $\begin{array}{c}\mathbf{R}_{\mathbf{p}} \\
\text { (GE IMS) }\end{array}$ & $\mathbf{R}_{\mathrm{p}}$ & $\begin{array}{c}\mathbf{R}_{\mathrm{pp}} \\
\text { (with RIP) }\end{array}$ & $\begin{array}{l}\text { LOD } \\
\text { (ng) }\end{array}$ & $\begin{array}{c}\text { LDR } \\
\text { (ng) }\end{array}$ \\
\hline 2,4-DNT & 6.03 & 1.57 & 14 & 15 & 6.2 & 3 & $3-25$ \\
\hline 2,4,6-TNT & 6.51 & 1.46 & 16 & 15 & 7.1 & 0.7 & $1-25$ \\
\hline RDX & 6.81 & 1.40 & 12 & 16 & 7.9 & 9 & $9-30$ \\
\hline Illicit Drugs & $\begin{array}{c}\text { Drift time } \\
\text { (msec) }\end{array}$ & $\frac{\mathbf{K}_{0}}{\left(\mathrm{~cm}^{2} \cdot \mathrm{V}^{-1} \mathrm{~s}^{-1}\right)}$ & $\begin{array}{c}\mathbf{R}_{\mathbf{p}} \\
\text { (GE IMS) }\end{array}$ & $\mathbf{R}_{\mathbf{p}}$ & $\mid \begin{array}{c}\mathbf{R}_{\mathrm{pp}} \\
\text { (with RIP) }\end{array}$ & $\begin{array}{l}\text { LOD } \\
\text { (ng) }\end{array}$ & $\begin{array}{l}\text { LDR } \\
\text { (ng) }\end{array}$ \\
\hline MDA & 8.76 & 1.39 & 11 & 15 & 6.7 & 5 & $5-50$ \\
\hline МА & 8.22 & 1.47 & 12 & 12 & 4.0 & 2 & $2-25$ \\
\hline BE & 10.95 & 1.10 & 12 & 11 & 5.6 & 8 & $8-75$ \\
\hline
\end{tabular}




\subsection{Future Improvements}

The results reported above illustrate the performance of the FIU IMS. There are several areas in which improvement on this first generation instrument is possible. The main components include the sample inlet nozzle, ion gate, and electronic shielding.

\subsubsection{Sample inlet for better transport of analytes}

The sample inlet made of aluminum is inexpensive, fast and easy to machine, but aluminum is not an ideal material if it is subjected to a surface deactivation process. The lack of deactivation results in a slow clearing cycle when analysis is performed on sticky analytes such as RDX, PETN, cocaine, etc. The FIU IMS requires 5-10 minutes to clear the RDX when the mass introduced is greater than $50 \mathrm{ng}$. A second generation inlet nozzle should be machined using stainless steel and the inner wall should be deactivated to avoid adsorption of the sample analytes on the surface.

\subsubsection{Ion gate to improve sensitivity and resolution}

The ion gate is one of the major components that determine the ion peak intensity and peak shape. There are many methods to which the $\mathrm{BN}$ gate can be constructed with varying cost and time. A BN gate can be designed to achieve best performance when; 1) the physical wire is small (fewer ions lost), 2) the distance between the alternating wires is minimal (lower offset voltage required for a sufficient gate electric field), and 3) the gate switching mechanism is fast (reduced peak tailing effect and ion loss).

The BN gate of the FIU IMS was constructed using a relatively simple approach to lower cost and time. In an improved version, a smaller wire can be used (i.e. reducing wire diameter from 0.01 " to 0.005 "). Wire and gate fixture materials must be selected to ensure the gate fixture 
and the gate wire has compatible expansion rates when heated to high temperature. The distance between wires can also be reduced, but if thin wires are used, they must always be in tension to avoid contact with the neighboring wires. An alternative fixture may be required to achieve such constraints.

The gate switch was inexpensively built (less than \$200) at the FIU electronic shop, and has a total rise and fall time of $10 \mu \mathrm{sec}$. Its switch rate is comparable to some older commercial IMS instruments. To further improve resolving power and sensitivity, a gate switch can be built to respond faster $(\sim 1 \mu \mathrm{sec})$ at higher cost. Alternatively, commercially available fast switch (nsec) can be purchased at a price of $\$ 2000$. Faster gate switching can afford analysis using smaller gate width of $\sim 50 \mu \mathrm{sec}$, which is a necessary specification for a high resolution IMS instrument.

\subsubsection{Electrical Shielding to further lower noise level}

Currently, the FIU IMS instrument is shielded at the detector end using a Faraday shield cup, and the drift tube is shielded with an aluminum enclosure. The noise level is acceptable when the instrument is operated at gain of $10^{\wedge} 9$ (baseline level at $\sim 50 \mathrm{mV}$ ). However, if required to be operated at gain of $10^{\wedge} 10$ (desirable for smaller gate width), the noise level must be improved. This can be achieved by shielding not just the IMS drift tube, but also the high voltage divider. The computer processing unit (CPU) can also be placed far away from the detector to avoid noise pickup from the cooling fan. Additionally, electronic components inside the main power box can also be redesigned to reduce noise from the power supply to the drift tube. 


\section{PART V: CONCLUSIONS}

\section{CHAPTER 16: PROJECT CONCLUSIONS}

16.1 Improving Detection of Hidden Explosives ............................................................ 197

16.2 Improving Detection of Hidden Illicit Drugs .........................................................198

16.3 Computer Modeling and Simulation Method for IMS Instrumental Design............198

16.4 Design and Construction of an IMS with Computer Controls ................................199

\subsection{Improving Detection of Hidden Explosives}

Air sampling and detection of plastic explosive vapors has always been a challenge as the result of the low vapor pressures of the energetic materials, RDX and PETN, that commercial IMS instruments are currently programmed to detect. This research study demonstrates an approach that targets more volatile, non-energetic compounds, such as taggants, decomposition products and/or impurities, rather than the explosives themselves. Successful vapor detection of plastic explosives was shown to be possible using IMS with operating conditions optimized to detect the volatile signatures of the explosives Detasheet (n-butyl acetate and DMNB), Semtex H (DMNB), and C-4 (DMNB and cyclohexanone). A SPME device was used to extract and preconcentrate the target volatile markers with sampling times in seconds to minutes as sufficient to extract and detect $\sim 20 \mathrm{ng}$ of the target analyte(s), which is 10 times the amount required for a reliable IMS response. SPME-IMS can greatly simplify the field sampling and detection process because SPME allows for remote air sampling without the need for additional cumbersome equipment and IMS provides rapid analysis at atmospheric pressure. Therefore, SPME-IMS has great potential to be a non-invasive, non-surface contact method for screening of low vapor pressure hidden explosives. Commercial dual-tube IMS analyzers can be set to have one drift 
tube operating at optimal conditions to detect the energetic explosives while the other drift tube can operate at optimal conditions to detect the non-energetic volatiles.

\subsection{Improving Detection of Hidden Illicit Drugs}

A headspace air sampling approach utilizing a SPME device to extract and preconcentrate targeted volatile markers for the successful detection of real drug samples by IMS was demonstrated for the first time. Detection of cocaine, MDMA, and marijuana via their targeted volatile markers was achieved within minutes of SPME sampling time, recovering low nanogram levels of the targeted analytes methyl benzoate, piperonal, and terpenes, respectively. Minimal peak interferences were observed in the 3 interference studies. However interferences from commonly encountered goods may pose some challenges for field detection, particularly when small quantities of drugs are sampled. Hence, improvement in the SPME selectivity is necessary in order to minimize the introduction of non-target analytes into the IMS.

\subsection{Computer Modeling and Simulation Method for IMS Instrumental Design}

Although the computer models incorporated several estimations and assumptions, SIMION/SDS simulations accurately predicted the resolution between two ion species when air was used for both the carrier and drift gas. The simulation results were in good agreement with the experimental results because the parameters used in the simulation closely matched the experimental conditions. As always, when modeling scientific instruments, the more accurately a simulation model matches the fabricated instrument and experimental conditions, the more accurately the predicted ion behavior will reflect reality. While SDS does have the limitation of only being able to accommodate one collision gas, the SIMION/SDS simulations did track the trends observed in the experiments for gas flow rates, drift gas composition, and gate width 
variations. The results of this first utilization of SIMION/SDS to simulate a complete IMS instrument are encouraging and future efforts should be able to take advantage of more advanced features in SIMION to overcome some of the identified modeling issues to produce more robust and flexible virtual IMS instruments. These results show that SIMION/SDS is a valuable tool for the development of IMS instruments and IMS-MS interfaces.

\subsection{Design and Construction of an IMS with Computer Controls}

Constructing an in-house IMS is common for research laboratories that require flexibility in changing experimental conditions and to test new designs or fundamental concepts. The FIU IMS is one of the first few in-house built IMS instruments with full computer control of the hardware and operating conditions. Each of the components fabricated for the first generation FIU IMS were based on conventional designs well documented in literature. Therefore, the FIU IMS performance is, as expected, comparable to other conventional designs. Similar to any other in-house IMS instruments, the FIU IMS provides the basic research platform for further research to be conducted, and is not meant to be compared to commercial instruments in all aspects unless new designs are to be created in the future. Since this instrument is the first one built at FIU, it was put together from scratch without an existing working instrument to test individual newly built components. Therefore, although engineering system integration procedures were followed, integration work was still the most challenging and time consuming step in the development of the FIU in-house IMS. 


\section{PART VI: FINAL REMARKS}

This dissertation reports the successful detection of compounds identified as dominant headspace components of explosives; Detasheet, Semtex H, and C-4, and illicit drugs; cocaine, MDMA, and marijuana using IMS. The two key contributions to this success were the use of a genetic algorithm as an optimization tool to reliably find the optimal IMS operating conditions for each of these compounds, and the availability of the SPME-IMS interface previously developed by our research group which allows the use of the commercial SPME fiber to effectively desorb the volatile compounds of explosives and illicit drugs for IMS analysis.

The results from this dissertation support the case for targeting volatile components as a method for presumptive detection of the presence of the bulk explosives and drugs. Moreover, this work describes an IMS specific genetic algorithm that can be used as an optimization tool for the detection and optimization of compounds of interest when no previous information about IMS detection is known for such specific compounds.

SPME greatly improves the probability of field detection of drugs and explosives, simplifies the detection process, and allows for rapid field sampling. SPME-IMS has a large range of potential applications besides detection of drugs and explosives, such as detection of chemicals and biohazards in industrial processes, cleanliness validation in pharmaceutical processes, and the detection of biomarkers for early disease detection. Once the compounds of interest are identified, researchers in these fields can apply the approach of SPME sampling and preconcentration prior to IMS detection, and optimization of the IMS parameters to achieve reliable and sensitive detection. 
It was also understood that detection of some compounds is not possible under the limited operating conditions provided by commercial IMS instruments. Depending on the chemical family and their properties, higher field strength or different ionization source etc. may be needed to form detectable ions. Therefore, one of the major efforts in this dissertation was the development of an in-house designed IMS that would allow future research to be conducted. The IMS constructed at FIU was conventional and of average performance, provides flexibility to control all parameters as well as providing a platform for testing of new component design concepts.

In addition to the in-house IMS instrument, effort was spent on development of the SIMION/SDS ion optics simulation method that would allow simulation of a complete IMS instrument to take place prior to construction. The SIMION/SDS simulation developed from this dissertation was the first reported simulation of a complete IMS instrument that accounts for the effect of the counter flow drift gas in a viscous environment. The simulation results were in excellent agreement with empirical data when the IMS drift gas is air. The SIMION/SDS method developed is a useful tool for the IMS community when designing new IMS instruments and interfaces, where optimizing gas flows, electric fields, and gate geometry is critical, and is especially important for the understanding of ion's behavior when a new component is to be fabricated and installed. 


\section{REFERENCES}

1. Affairs, U.S.C.S.C.o.H.S.a.G., National Bombing Prevention Act of 2007 report of the Committee on Homeland Security and Governmental Affairs. 2008, Washington: U.S. G.P.O.

2. United Nations News Center, Ban hails 'life-saving work' of eliminating landmines on International Day. Accessed April 2009, http://www.un.org/apps/news/story.asp?NewsID=30397\&Cr=landmines\&Cr1=.

3. United Nations Office on Drugs and Crime, World Drug Report. 2009, p. 314.

4. Beveridge, A.., Forensic Investigation of Explosions. Forensic Science Series, ed. 1998, Taylor \& Francis, Bristol PA.

5. Yinon, J., Advances in Analysis and Detection of Explosives. 1993, Kluwer Academic Publishers, Norwell, MA.

6. Adams, C., CATS at the Airport. Accessed April 2009.

7. TSA, Millimeter Wave Scanner, Whole Body Imaging. Accessed April 2009, http://www.tsa.dhs.gov/approach/tech/body_imaging.shtm.

8. Berrick, C.A., TSA's Explosives Detection Canine program status of increasing number of explosives detection canine teams. 2008, Washington, DC : U.S. Govt. Accountability Office. Govdoc no.: GA 1.41: GAO-08-933 R.

9. Kapoor, J.C., Kannan, G.K., Landmine Detection Technologies to Trace Explosive Vapor Detection Techniques. Defense Science Journal, 2007. 57(6): p. 797-810.

10. Yang, X., Du, X.X., Shi, J., Swanson, B., Molecular recognition and self-assembled polymer films for vapor phase detection of explosives Talanta, 2001: p. 439-445.

11. Goodpaster, J.V., McGuffin, V.L., Fluorescence quenching as an indirect detection method for nitrated explosives. Analytical Chemistry, 2001. 73(9): p. 2004-2011.

12. Toal, S.J., Trogler, W.C., Polymer sensors for nitroaromatic explosives detection. Journal of Materials Chemistry, 2006. 6: p. 2871-2883.

13. Thomas, S.W, III, Amara, J.P., Bjork, R.E., Swager, T.M., Amplifying fluorescent polymer sensors for the explosives taggant 2,3-dimethyl-2,3-dinitrobutane. Chem. Commun., 2005: p. $4572-4574$.

14. Pinnaduwage, L.A., Hedden, D.L., Gehl, A., Boiadjiev, V.I., Hawk, J.E., Farahi, R.H., Thundat, T., A sensitive, handheld vapor sensor based on microcantilevers. Review of Scientific Instruments, 2004. 75(11): p. 4554-4557. 
15. Pinnaduwage, L.A., Boiadjiev, V., Hawk, J.E., Thundat T., Sensitive detection of plastic explosives with self-assembled monolayer-coated microcantilevers. Applied Physics Letters, 2003. 83(7): p. 1471-1473.

16. Krausa, M., Reznev A.A., Vapour and trace detection of explosives for anti-terrorism purposes. Nato Science Series, 2004, Kluwer Academic Publishers, Norwell, MA.

17. Eiceman, G.A., Karpas, Z., Ion Mobility Spectrometry. Second Edition. 2005, CRC Press, Boca Raton, FL.

18. Lorenzo, N., Wan, T., Harper, R.J., Hsu, Y.L., Chow, M., Rose, S., Furton, K.G., Laboratory and field experiments used to identify Canis lupus var. familiaris active odor signature chemicals from drugs, explosives, and humans. Anal Bioanal Chem 2003. 376: p. 1212-1224.

19. Ewing, R.G., Atkinson, D.A., Eiceman, G.A., Ewing, G.J., A critical review of ion mobility spectrometry for the detection of explosives and explosive related compounds. Talanta, 2001. 54(3): p. 515-529.

20. SWGDOG SC8-Substance Detector Dogs Narcotics Section. 2007 (Accessed 03/10/08). http://www.swgdog.org/.

21. Eiceman, G.A., Karpas, Z., Ion Mobility Spectrometry. Second Edition. 2005, CRC Press, Boca Raton, FL.

22. Hallowell, S.F., Screening people for illicit substances: a survey of current portal technology. Talanta, 2001. 54(3): p. 447-458.

23. Yinon, J., Forensic Applications of Mass Spectrometry. II. Vol. 3. 1995, CRC Press, Boca Raton, FL.

24. United Nation, Convention on the Marking of Plastic Explosives for the Purpose of Identification. 1991.

25. Yinon, J., Forensic and environmental detection of explosives. 1999, John Wiley \& Sons Ltd. New York, NY.

26. Perr, J.M., K.G. Furton, and J.R. Almirall. Application of a SPME-IMS Detection System for Explosives Detection in Sensors, and Command, Control, Communications, and Intelligence Technologies for Homeland Defense and Law Enforcement. in Pro. SPIE - The Int. Soc. for Optical Engineering. 2005. E.M. Carapezza, Ed.

27. Perr, J.M., Furton, K.G., Almirall, J.R., Solid phase microextraction ion mobility spectrometer interface for explosive and taggant detection. J. Separation Science, 2005. 28(2): p. 177-183. 
28. Guerra, P., Lai, H., Almirall, J.R., Analysis of the volatile chemical markers of explosives using novel solid phase microextraction coupled to ion mobility spectrometry. J. of Separation Science, 2008. 31: p. 2891-2898.

29. Lai, H., Corbin, I., Almirall, J.R., Headspace sampling and detection of cocaine, MDMA, and marijuana via volatile markers in the presence of potential interferences by SPME-IMS. Anal Bioanal Chem, 2008. 392: p. 105-113.

30. Lai, H., Guerra, P., Joshi, M., Almirall, R.J., Analysis of volatile components of drugs and explosives by solid phase microextraction-ion mobility spectrometry. J. Separation Science, 2008. 31(2): p. 402-412.

31. L. Paul Waggoner, et al., Canine olfactory sensitivity to cocaine hydrochloride and methyl benzoate. Proc. SPIE, 1997. p2937.

32. Lorenzo, N., Wan, T., Harper, R.J., Hsu, Y.L., Chow, M., Rose, S., Furton, K.G., Laboratory and field experiments used to identify Canis lupus var. familiaris active odor signature chemicals from drugs, explosives, and humans. Anal Bioanal Chem 2003. 376: p. 1212-1224.

33. Harper, R.J., Almirall, J.R., Furton, K.G., Identification of dominant odor chemicals emanating from explosives for use in developing optimal training aid combinations and mimics for canine detection. Talanta, 2005. 67(2): p. 313-327.

34. Furton, K.G., Myers L.J., The scientific foundation and efficacy of the use of canines as chemical detectors for explosives. Talanta, 2001. 54(3): p. 487-500.

35. Lawrence, A.H., Neudorfi, P., Detection of ethylene glycol dinitrate vapors by ion mobility spectrometry using chloride reagent ions. . Analytical Chemistry, 1988. 60: p. 104-109.

36. Ewing, R.G., Miller, C.J., Detection of Volatile Vapors Emitted from Explosives with a Handheld Ion Mobility Spectrometer. Field Analytical Chemistry and Technology, 2001. 5(5): p. 215-221.

37. Gahlinger, P.M., Illegal Drugs, A complete guide to their history, chemistry, use, and abuse. 2004, Plume.

38. Cole, M.D., The Analysis of Controlled Substances. Analytical Techniques in the Sciences. 2003, John Wiley \& Sons Ltd., New York, NY.

39. Waddell-Smith, R.J.H., A Review of Recent Advances in Impurity Profiling of Illicit MDMA Samples. J Forensic Sci, 2007. 52(6): p. 1297-1304.

40. Cheng, W.C., Poon, N.L., Chan, M.F., Chemical profiling of 3,4methylenedioxymethamphetamine (MDMA) tablets seized in Hong Kong. J Forensic Sci., 2003. 48(6): p. 1249-1259. 
41. Waddell-Smith, R.J.H. Optimization of Headspace Solid Phase Microextraction (HSSPME) for Organic Impurity Profiling of Illicit MDMA tablets. Annual Meeting of the American Academy of Forensic Sciences 2008. Washington, DC.

42. Howard, P.H., Handbook of Environmental Fate and Exposure Data for Organic Chemicals. Vol. 5. 1997, CRC Press, Boca Raton, FL.

43. Lukaszewski, T., Jeffery, W.K., Impurities and Artifacts of Illicit Cocaine. Journal of Forensic Sciences, 1980. 25(3): p. 499-507.

44. Su, C.-W., Columnless GC/IMS (II) - A novel on-line separation technique for IonScan analysis. International Journal for Ion Mobility Spectrometry, 2002. 5(2): p. 160-174.

45. Furton, K.G., Hong, Y.-C., Hsu, Y.-L., Luo, T., Rose, S., Walton, J., Identification of Odor Signatures Chemicals in Cocaine Using Solid-Phase Microextraction-Gas Chromatography and Detector-Dog Response to Isolated Compounds Spikes on U.S. Currency. Journal of Chromatographic Science, 2002. 40(3): p. 147-155.

46. Staples, E.J., Viswanathan, S., Detection of Contrabands in Cargo Containers Using a HighSpeed Gas Chromatograph with Surface Acoustic Wave Sensor. Ind. Eng. Chem. Res., 2008. 47(21): p. 8361-8367.

47. Lindy E. Dejarme, et al., The formation of methyl benzoate from cocaine hydrochloride under different temperatures and humidities. Proc. SPIE, 1997. 2937.

48. Lindy E. Dejarme, et al., Analysis of the volatile organic compounds in seized cocaine hydrochloride Proc. SPIE, 1997. 2937.

49. Neudorfi, P., et al., Detection of cocaine in cargo containers by high-volume vapor sampling. Field test at Port of Miami. Proc. SPIE, 1997. 2937: p. 26-34.

50. Karukstis, K.K., Hecke, G.R.V., Chemistry Connections: The Chemical Basis of Everyday Phenomena. 2 ed. Complementary Science Series. 2003, Academic Press, Burlington, MA.

51. Neudorfl, P., Hupé, M., Pilon, P., Lawrence, A.H., Determination of Ecgonidine Methyl Ester Vapor Pressure Using a Dynamic Gas Blending System and Gas Chromatographic Analysis. Analytical Chemistry, 1997. 69(20): p. 4283-4285.

52. Clement, R.E. Siu, K.W.M, Hill, H.H., Instrumentation for Trace Organic Monitoring. 1992, Lewis Publishers Inc., Martin, SD.

53. Langeveld, W.G.J., Johnson, W.A., Owen, R.D., Schonberg, R.G., Intensity-Modulated Advanced X-Ray Source (IMAXS) for Homeland Security Applications. IEEE Transactions on Nuclear Science. 2009. 56(3): p. 1288-1291.

54. Buffler, A., Contraband detection with fast neutrons. Radiation Physics and Chemistry 2004. 71(3-4): p. 853-861. 
55. Lu, M., Shen, J., Li, N., Zhang, Y., Zhang, C., Liang, L., Xu, X., Detection and Identification of Illicit Drugs using Terahertz Imaging. Journal of Applied Physics, 2006. 100(10): p. 103104-103104-5.

56. Stubbs, D.D., Lee, S.-H., Hunt, W.D., Investigation of Cocaine Plumes Using Surface Acoustic Wave Immunoassay Sensors. Anal. Chem., 2003. 75(22): p. 6231-6235.

57. Oxley, J.C., Waggoner, L.P., Aspects of Explosives Detection. 2009, Elsevier Science Ltd., Kidlington, Oxford.

58. Voiculescu, I., McGill, R.A., Zaghloul, M.E., Mott, D., Stepnowski, J., Stepnowski, S., Summers, H., Nguyen, V., Ross, S., Walsh, K., Martin, M., Microprecontrator for Enhanced Trace Detection of Explosives and Chemical Agents. IEEE Sensors Journal, 2006. 6(5): p. 1094-1102.

59. Cotte-Rodríguez, I., Handberg, E., Noll, R.J., Kilgour, D.P.A, Cooks, R.G., Improved detection of low vapor pressure compounds in air by serial combination of single-sided membrane introduction with fiber introduction mass spectrometry (SS-MIMS-FIMS). Analyst, 2005. 130: p. 679-686.

60. Linker, K.L., Conrad, F.J., Custer, C.A., Rhykerd, C.L. Jr., Particle preconcentrator, U.S. Patent, Editor. 2005, Sandia National Laboratories: USA.

61. Linker, K.L., Bouchier, F.A., Theisen, L., Arakaki, L.H., Analyte separation utilizing temperature programmed desorption of a preconcentrator mesh, U.S. Patent, Editor. 2007, Sandia Corporation: USA.

62. Martin, M., Crain, M., Walsh, K., McGill, R.A., Houser, E., Stepnowski, J., Stepnowski, S., Wu, H.-D., Stuart, R., Microfabricated vapor preconcentrator for portable ion mobility spectrometry. Sensors and Actuators B, 2007. 126: p. 447-454.

63. Senesac, L., Thundat, T.G., Nanosensors for explosives detection. Materials Today, 2008. 11(3): p. 28-36.

64. Pawliszyn, J., Solid Phase Microextraction: Theory and Practice 1997 Wiley-VCH Inc., New York, NY.

65. Yeom, J., Field, C.R., Bae, B., Masel, R.I., Shannon, M.A., The design, fabrication and characterization of a silicon microheater for an integrated MEMS gas preconcentrator. J. Micromech. Microeng., 2008. p. 125001.1-125001.12.

66. Ronald P. Manginell, et al., Mass-Sensitive Microfabricated Chemical Preconcentrator Journal of Microelectromechanical Systems, 2008. 17(6): p. 1396-1407.

67. Alfeeli, B., Cho, D., Ashraf-Khorassani, M., Taylor, L.T., Agah, M., MEMS-based multiinlet/outlet preconcentrator coated by inkjet printing of polymer adsorbents. Sensors and Actuators B, 2008. 133(1): p.24-32. 
68. Siegal, M.P., Overmyer, D.L., Kottenstette, R.J., Tallant, D.R., Yelton, W.G., Nanoporouscarbon films for microsensor preconcentrators. Applied Physics Letters, 2002. 80(21): p. 3940.

69. Arce, L., Menéndeza, M., Garrido-Delgadoa, R., Valcárcel, M., Sample-introduction systems coupled to ion-mobility spectrometry equipment for determining compounds present in gaseous, liquid and solid samples. Trends in Analytical Chemistry, 2008. 72(2): p. $139-150$.

70. Spangler, G.E., Carrico, J.P., Membrane inlet for ion mobility spectrometry (plasma chromatography). International Journal of Mass Spectrometry and Ion Physics, 1983. 52(23): p. 267-287.

71. Kotiaho, T., Lauritsen, F.R., Degn, H., Paakkanen, H., Membrane inlet ion mobility spectrometry for on-line measurement of ethanol in beer and in yeast fermentation. Anal. Chim. Acta 1995. 309: p. 317-325.

72. Spangler, G.E., Carrico, J.P., Membrane inlet for ion mobility spectrometry (plasma chromatography). Int. J. Mass Spectrom. Ion Process, 1983. 52: p. 267-287.

73. Kabarle, P., Tang, L., From ions in solution to ions in the gas phase. Analytical Chemistry, 1993. 65: p. 972A-986A.

74. Asbury, G.R., Klasmeier, J., Hill, H.H. Jr., Analysis of explosives using electrospray ionization: ion mobility spectrometry (ESI:IMS). Talanta, 2000. 50: p. 1291-1298.

75. Matz, L.M., Hill, H.H. Jr., Evaluation of Opiate Separation by High-Resolution Electrospray Ionization-Ion Mobility Spectrometry/Mass Spectrometry. Analytical Chemistry, 2001. 73: p. 1664.

76. Matz, L.M., Hill, H.H. Jr., Evaluating the Separation of Amphetamines by Electrospray Ionization Ion Mobility Spectrometry/MS and Charge Competition within the ESI Process. Anal. Chem., 2002. 74: p. 420.

77. Tabrizchi, M., Khayamian, T., Taj, N., Design and optimization of a corona discharge ionization source for ion mobility spectrometry. Review of Scientific Instruments, 2000. 71(6): p. 2321-2328.

78. Khayamian, T., Tabrizchi, M., Jafari, M.T., Analysis of 2,4,6-trinitrotoluene, pentaerythritol tetranitrate and cyclo-1,3,5-trimethylene-2,4,6-trinitramine using negative corona discharge ion mobility spectrometry. Talanta, 2003. 59: p. 327.

79. Sielemann, S., Baumbach, J.I., Schmidt, H., Pilzecker, P., Detection of alcohols using UVion mobility spectrometers. Analytica Chimica Acta, 2001. 431: p. 293-301. 
80. Xie, Z., Sielemann, S., Schmidt, H., Li, F., Baumbach, J.I., Determination of acetone, 2butanone, diethyl ketone and BTX using HSCC-UV-IMS. Anal Bioanal Chem, 2002. 372: p. 606-610.

81. Rasulev, U.Kh., Iskhakova, S.S., Khasanov, U., Mikhailin, A.V., Atmosphere Pressure Surface Ionization Indicator of Narcotics. International Journal for Ion Mobility Spectrometry, 2001(2): p. 121-125.

82. Michels, A., Tombrink, S., Vautz, W., Miclea, M., Franzke, J., Spectroscopic characterization of a microplasma used as ionization source for ion mobility spectrometry. Spectro Chimica Acta Part B 2007. 62: p. 1208-1215.

83. Vautz, W., Michels, A., Franzke, J., Micro-plasma: a novel ionization source for ion mobility spectrometry. Anal Bioanal Chem 2008. 391: p. 2609-2615.

84. Eiceman, G.A., Vandiver, V.J., Leasure, C.S., Anderson, G.K., Tiee, J.J., Danen, W.C., Effects of Laser Beam Parameters in Laser Ion Mobility Spectrometry. Analytical Chemistry, 1988. 58: p. 1690-1695.

85. Lubman, D.M., Kronick, M.N., Plasma Chromatography with Laser-Produced Ions. Analytical Chemistry, 1982. 54(9): p. 1546-1551.

86. Wang, Y., Nacson, S., Pawliszyn, J., The coupling of solid-phase microextraction/surface enhanced laser desorption/ionization to ion mobility spectrometry for drug analysis. Analytica Chimica Acta 2007. 582(1): p. 50-54.

87. Yoon, O.K., Zuleta, I.A., Robbins, M.D., Barbula, G.K., Zare, R.N., Simple TemplateBased Method to Produce Bradbury-Nielsen Gates. J Am Soc Mass Spectrom, 2007. 18(11): p. 1901-1908.

88. Szumlas, A.W., Rogers, D.A., Hieftje, G.M., Design and construction of a mechanically simple, interdigitated-wire ion gate. Review of Scientific Instruments, 2005.76: p.086108-1.

89. Kimmel, J.R., Engelke, F., Zare, R.N., Novel Method for the Production of Finely Spaced Bradbury-Nielsen Gates. . Rev. Sci. Instrum. , 2001. 72: p. 4354.

90. Zuleta, I.A., Barbula, G.K., Robbins, M.D., Yoon, O.K., Zare, R.N., Micromachined Bradbury-Nielsen Gates. Anal. Chem., 2007. 79(23): p. 9160-9165.

91. Knorr, F.J., Eatherton, R.L., Siems, W.F., Hill, H.H.Jr., Fourier Transform Ion Mobility Spectrometry. Anal. Chem., 1985. 57(2): p. 402-406.

92. Tarver, E.E., External second gate, Fourier transform ion mobility spectrometry: parametric optimization for detection of weapons of mass destruction. Sensors, 2004. 4: p. 1-13.

93. Spangler, G.E., Expanded theory for the resolving power of a linear ion mobility spectrometer International Journal of Mass Spectrometry, 2002. 220(3): p. 399-418. 
94. Eiceman, G.A., Nazarov, E.G., Rodriguez, J.E., Analysis of a drift tube at ambient pressure: Models and precise measurements in ion mobility spectrometry. Review of Scientific Instruments, 2001. 72(9): p. 3610-3621

95. Pfeifer, K.B., Sanchez, R.C., Miniaturized IMS System for Explosives and Contraband Detection. International Journal of Ion Mobility Spectrometry, 2002. 5(3): p. 63-66.

96. Guevremont, R., Purves, R.W., Atmospheric pressure ion focusing in a high-field asymmetric waveform ion mobility spectrometer. Review of Scientific Instruments, 1999. 70(2): p.1370-1384.

97. Miller, R.A., Eiceman, G.A., Nazarov, E.G., King, A.T., A novel micromachined high-field asymmetric waveform-ion mobility spectrometer. Sensors and Actuators B, 2000. 67(3): p. 300-306.

98. Garner-O'Neale, L.D., Bonamy, A.F., Meek, T.L., Patrick, B.G., Calculating group electronegativities using the revised Lewis-Langmuir equation. Journal of Molecular Structure (Theochem), 2003. 639(3): p. 151-156.

99. CRC Handbook, Proton Affinity. (Accessed January 2007) Vol.10. p. 173-191.

100. Eiceman, G.A., Ion mobility spectrometry as a fast monitor of chemical composition. Trends in analytical chemistry, 2002. 21(4): p. 259-275.

101. Buttigieg, G.A., Knight, A.K., Denson, S., Pommier, C., Denton, M.B., Characterization of the explosive triacetone triperoxide and detection by ion mobility spectrometry. Forensic Science International, 2003. 135(1): p. 53-59.

102. Ewing, R.G., Eiceman, G.A., Stone, J.A., Proton-bound cluster ions in ion mobility spectrometry. International Journal of Mass Spectrometry, 1999. 193(1): p. 57-68.

103. Ewing R. G., Eiceman, G.A., Harden, C.S., Stone, J.A., The kinetics of the decompositions of the proton bound dimers of 1,4-dimethylpyridine and dimethyl methylphosphonate from atmospheric pressure ion mobility spectra. International Journal of Mass Spectrometry, 2006. 255-256 (1-3): p. 76-85

104. Eiceman, G.A., Nazarov, E.G., Rodriguez, J. E., Chemical class information in ion mobility spectra at low and elevated temperatures. Analytica Chimica Acta 2001. 433(1): p. 53-70.

105. Tabrizchi, M., Rouholahnejad, F., Pressure effects on resolution in ion mobility spectrometry. Talanta, 2006. 69(1): p. 87-90.

106. Soppart, O., Baumbach, J.I., Comparison of electric fields within drift tubes for ion mobility spectrometry. Measurement Science \& Technology, 2000. 11(10): p. 1473-1479. 
107. Eiceman, G.A., Leasure, C.S., Vandiver, V.J., Rico, G., Flow characteristics in a segmented closed-tube design for ion mobility spectrometry. Analytica Chimica Acta, 1985. 175: p. 135-145.

108. Asbury, G.R., Hill, H.H., Using Different Drift Gases To Change Separation Factors $(\alpha)$ in Ion Mobility Spectrometry. Analytical Chemistry 2000. 72(3): p. 580-584.

109. Mariano, A.V., Su, W., Guharay, S.K., Effect of Space Charge on Resolving Power and Ion Loss in Ion Mobility Spectrometry. Analytical Chemistry, 2009. 81(9): p. 3385-3391.

110. Tolmachev, A.V., Clowers, B.H., Belov, M.E., Smith, R.D., Coulombic Effects in Ion Mobility Spectrometry. Analytical Chemistry, 2009. 81(12): p. 4778-4787.

111. Bell, S., Nazarov, E., Wang, Y.F., Eiceman, G.A., Classification of ion mobility spectra by functional groups using neural networks. Analytica Chimica Acta, 1999. 394: p. 121-133.

112. Scott, J.R., Tremblay, P.L., Noncontact measurement of electrostatic fields: Verification of modeled potentials within ion mobility spectrometer drift tube designs. Rev. Sci. Instrum., 2007. 78(3): p. 035110-035114.

113. Vautz, W., Sielemann, S., Baumbach, J.I., 3D gas flow simulation as a tool for the characterization and optimization of ion mobility spectrometers. International Journal for Ion Mobility Spectrometry, 2004. 7(1): p. 19-24.

114. Dahl, D.A., Delmore, J. E., Appelhans, D. J., SIMION PC/PS2 electrostatic lens design program. Rev. Sci. Instrum., 1990. 61(1): p. 607-609.

115. Dahl, D.A., SIMION 3D Version 7.0 User's Manual, Idaho National Engineering and Environmental Laboratory, Idaho Falls, ID, 2000, Bechtel BWXT Idaho, LLC.

116. Dahl, D.A., SIMION for the personal computer in reflection. Int. J. Mass Spectrom., 2000. 200(1): p. 3-25.

117. American Mass Spectrometry Society, (Accessed February 04, 2008).

118. Veryovkin, I.V., Calaway, W.F., Pellin M.J., A virtual reality instrument: Near-future perspective of computer simulations of ion optics. Nuclear Instruments and Methods in Physics Research, Section A: Accelerators, Spectrometers, Detectors and Associated Equipment. , 2004. 519(1-2): p. 363-372.

119. Appelhans A.D., Dahl, D.A., SIMION ion optics simulations at atmospheric pressure. International Journal of Mass Spectrometry, 2005. 244(1): p. 1-14.

120. Hunka, D.E., Austin, D.E., Sandia Report SAND2005-6908: Ion Mobility Spectrometer / Mass Spectrometer (IMS-MS), U.S.D.o.E. Sandia National Laboratories, Editor. 2005. 
121. Dahl, D.A, McJunkin, T.R., Scott, J.R., Comparison of ion trajectories in vacuum and viscous environments using SIMION: Insights for instrument design. Int. J. Mass Spectrom., 2007. 266(1-3): p. 156-165.

122. Spangler, G.E., Collins, C.I., Peak Shape Analysis and Plate Theory for Plasma Chromatography. Analytical Chemistry, 1975. 47(3): p. 403-407.

123. Ching $\mathrm{Wu}$, et al., Construction and characterization of a high-flow, high-resolution ion mobility spectrometer for detection of explosives after personnel portal sampling. Talanta, 2002. 57(1): p. 123-134.

124. Cohen, M.J., Karasek, F.W., Plasma Chromatography-a new dimension for gas chromatography and mass spectrometry. Journal of Chromatography Science, 1970. 8(6): p. 330-337.

125. Karasek, F.W., Kane, D.M., Ionic species of organic compounds observed in mobility spectra by plasma chromatography. Journal of Chromatography, 1974. 93(1): p. 129-139.

126. Revercomb, H.E., Mason, E.A., Theory of plasma chromatography / gaseous electrophoresis: a review. Analytical Chemistry, 1975. 47(7): p. 970-983.

127. Baumbach, J.I., Eiceman, G.A., Ion mobility spectrometry: arriving on site and moving beyond a low profile. Applied spectroscopy 1999. 53(9): p. 338A-353A.

128. Sakawa, M., Genetic algorithms and fuzzy multiobjective optimization. 2002: Boston : Kluwer Academic Publishers, Chapter 2. 288 pages.

129. Vose, M.D., The simple genetic algorithm : foundations and theory. 1999: Cambridge, MA: MIT Press, 251 pages.

130. Deb, K., Multi-objective optimization using evolutionary algorithms. 2001, New York : John Wiley \& Sons, New York, Chapter 4.2. 518 pages.

131. Daum, K.A., Atkinson, D.A., Ewing, R.G., Knighton, W.B., Grimsrud, E.P., Resolving interferences in negative mode ion mobility spectrometry using selective reactant ion chemistry. Talanta, 2001. 54(3): p. 299-306.

132. Bradbury, N.E., Nielsen, R.A., Absolute values of the electron mobility in hydrogen. Physical Review, 1936. 49(5): p. 388-393.

133. Asbury, G.R., Hill, H.H.Jr., Evaluation of Ultrahigh Resolution Ion Mobility Spectrometry as an Analytical Separation Device in Chromatographic Terms. Journal of Microcolumn Separations, 2000. 12(3): p. 172-178.

134. Hill, H.H.Jr., IMS Short Course, July 2006. Honolulu, Hawaii.

135. Lorenzo, N., Florida International University MSFS Thesis, 2002. 
136. Pierre, D.A., Optimization Theory with Applications. 1969: John Wiley \& Sons, Inc., New York, Chapter 5.5. 612 pages.

137. Lasdon, L.S., Optimization Theory for Large System. 1970: MacMillan Publishing Co., Inc. New York, Chapter 1.2. 523 pages.

138. Grabitech, MultiSimplex 2.1 Software.

139. Drolet, G., Trace vapor detection, in Patent Storm, U.S. Patent, Editor. 1999, Revenue Canada: USA. p. 32.

140. Collins, D.C., Lee, M.L., Developments in ion mobility spectrometry-mass spectrometry. Anal Bioanal Chem, 2002. 372: p. 66.

141. Jo Nell Aarons, Master Thesis 2008, Florida International University.

142. Vautz, W., Baumbach, J.I., Uhde, E., Detection of emissions from surfaces using ion mobility spectrometry. Anal Bioanal Chem 2006. 384: p. 980.

143. World Health Organization, Butanols: Four isomers. Environmental Health Criteria, 1987. Vol. 65.

144. Merory, J., Food Flavorings: Composition, Manufacture and Use. 2nd ed. 1968, AVI Publishing Company, Inc., Westport, CT.

145. Matz, L.M., Hill, H.H.Jr., Beegle, L.W., Kanik, I., Investigation of drift gas selectivity in high resolution ion mobility spectrometry with mass spectrometry detection. Journal of the American Society for Mass Spectrometry, 2002. 13(4): p. 300-307.

146. Mesleh, M.F., Hunter, J.M., Shvartsburg, A.A., Schatz, G.C., Arrold, M.F., Structural information from ion mobility measurements: Effects of the long-range potential. J. Phys. Chem, 1996. 100: p. 16082-16086. 


\section{APPENDICES}

\section{APPENDIX I. GA PROGRAMMING FILE ON EXCEL MACROS}

\section{GENERATE NEW CHROMOSOMES}

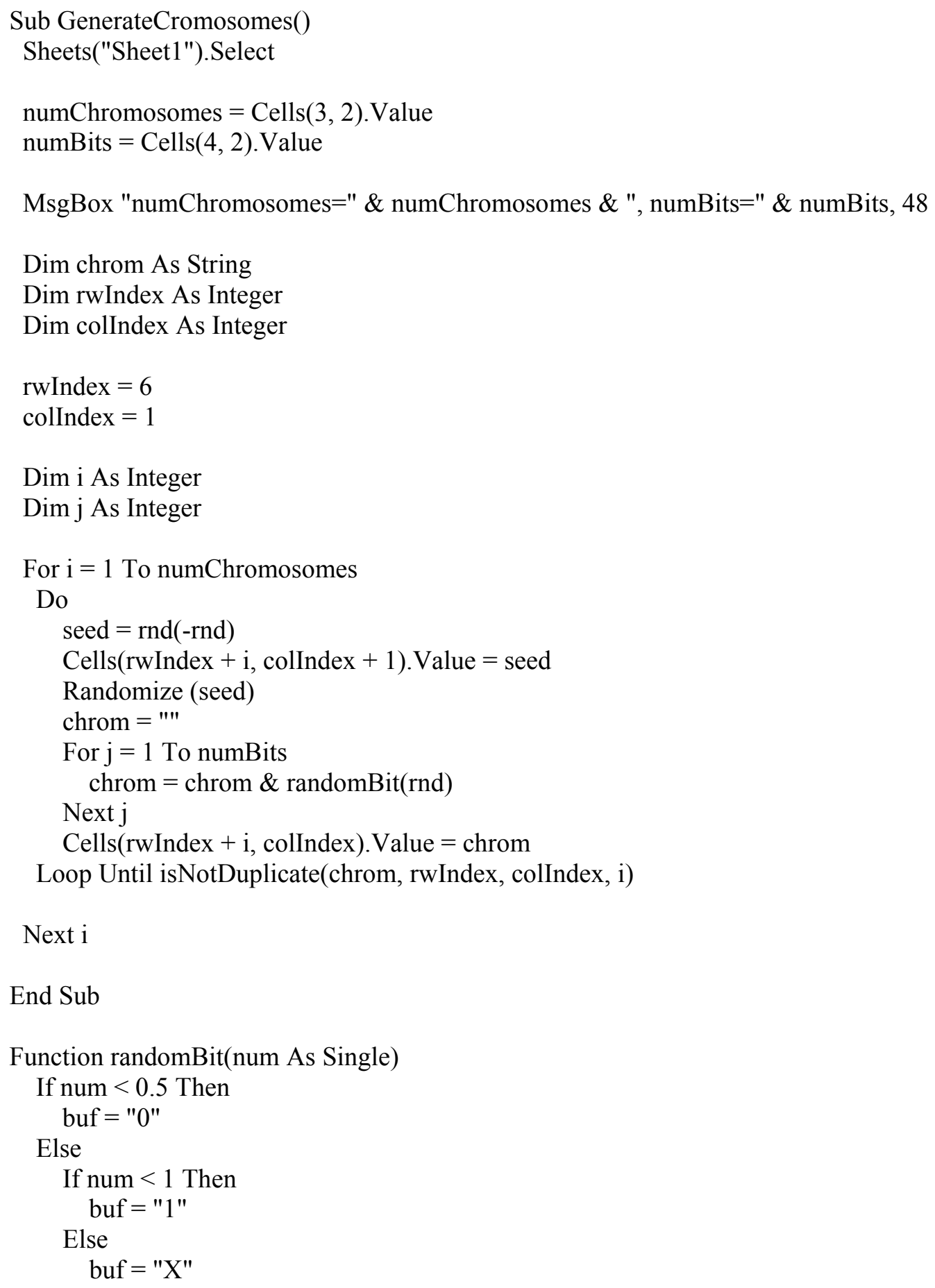


End If

End If

randomBit $=$ buf

End Function

Function isNotDuplicate(chromosome As String, rwIndex As Integer, colIndex As Integer, numChromosomes As Integer)

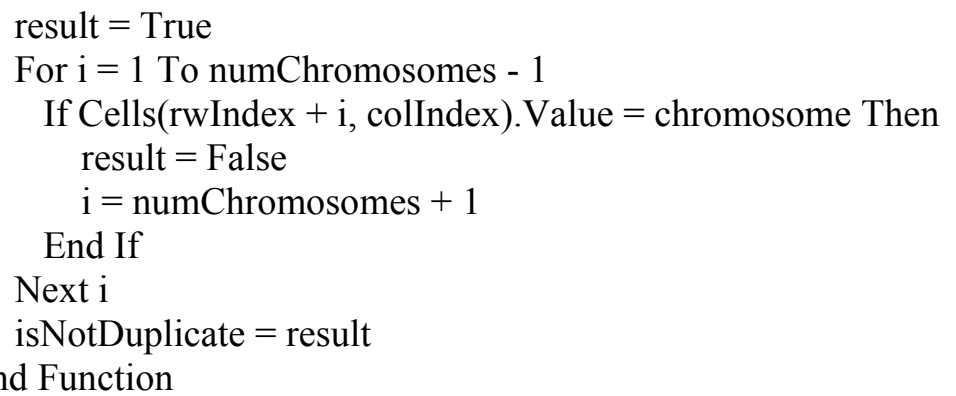

\section{GENETIC ALGORITHMS}

Sub SortPopulation()

$$
\begin{aligned}
& \text { populationRow }=6 \\
& \text { populationCol }=1 \\
& \text { numChromosomes }=\text { Cells }(3,2) . \text { Value } \\
& \text { Dim StartRowIndex As String } \\
& \text { Dim EndRowIndex As String } \\
& \text { Dim populationRange As String } \\
& \text { Dim sortKey As String }
\end{aligned}
$$

StartRowIndex $=$ populationRow +1

EndRowIndex $=$ populationRow + numChromosomes +1

populationRange = "A" + StartRowIndex + ":B" + EndRowIndex

Worksheets("Sheet1").Range(populationRange).Sort Key1:=Worksheets("Sheet1").Range("B" + StartRowIndex)

End Sub

Sub NewMatingPool()

'Make sure the pool is sorted before running SortPopulation

matingpoolRow $=6$

matingpoolCol $=4$ 


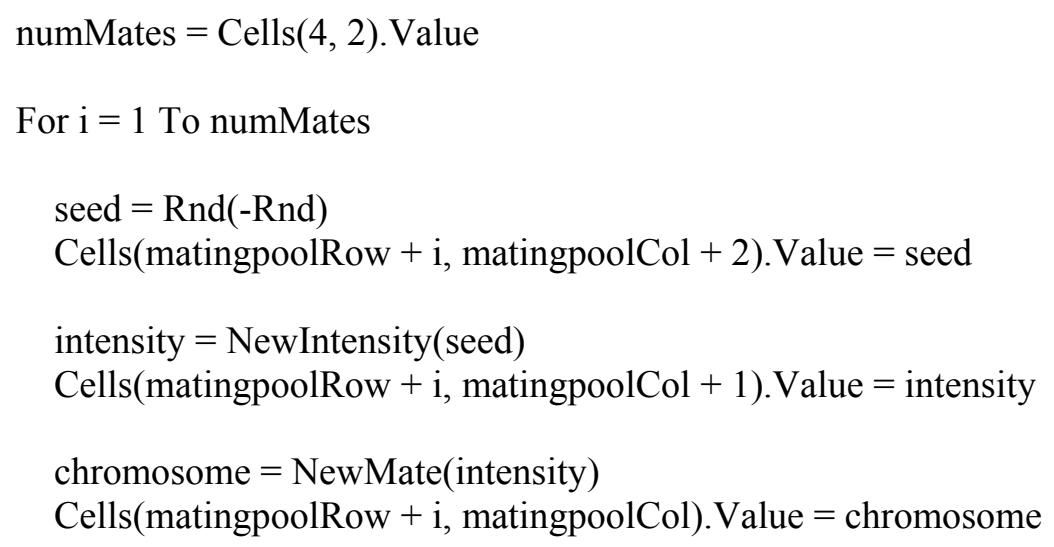

Next i

End Sub

Sub NewChildren()

'Make sure the pool is sorted before running SortPopulation

Dim newChildRow As Integer

Dim newChildCol As Integer

newChildRow $=6$

newChildCol $=8$

numChildren $=$ Cells $(5,2)$. Value

numBits $=\operatorname{Cells}(2,2)$.Value

Dim i As Integer

For $\mathrm{i}=1$ To numChildren -1

'select 2 chromosomes via roulette

seed $=$ Rnd(-Rnd)

Randomize (seed)

Dim c1 As String

Dim c2 As String

c1 $=$ NewMate $($ NewIntensity $($ Rnd $))$

c2 = NewMate(NewIntensity(Rnd))

'crossover to get 2 new chromosomes

childrenstring $=$ Crossover $(\mathrm{c} 1, \mathrm{c} 2)$

Dim new1 As String

Dim new2 As String

'cut the childrenstring (returned above) into its two parts new1 $=$ Mid(childrenstring, 1 , numBits) 


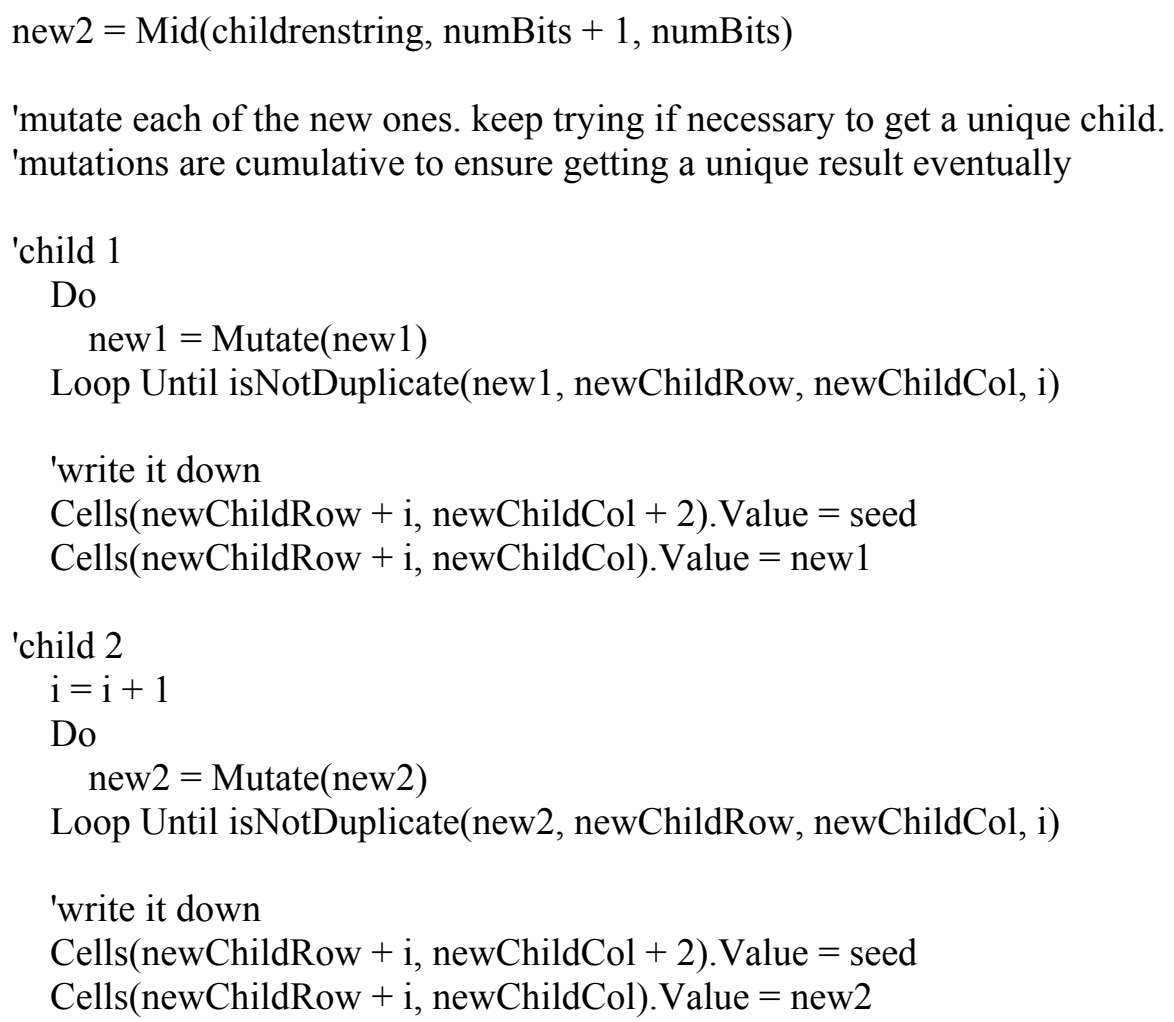


numChromosomes $=$ Cells $(3,2)$.Value

Dim i As Integer

For $\mathrm{i}=1$ To numChromosomes

If Cells(rwIndex $+\mathrm{i}$, colIndex +1 ). Value $>$ intensity Then

'here the function returns the selected chromosome based on the intensity

NewMate $=$ Cells $($ rwIndex $+i$, colIndex $)$.Value

'this makes the loop stop by making i too big to trigger another iteration $\mathrm{i}=$ numChromosomes +1

End If

Next $\mathrm{i}$

End Function

Function Crossover(c1 As String, c2 As String)

'123456789 \& abcdefghi become 123def789 \& abc456ghi

'fix this to make it general w.r.t. the chromosome length (numBits below)

$\operatorname{Dim} \operatorname{Arr}(2)$

numBits $=$ Cells $(2,2)$. Value

Dim new1 As String

Dim new2 As String

'if change \# of chromosomes, edit these two lines

'the function mid works like this:

'Mid(String, Start , [Length])

new1 $=\operatorname{Mid}(\mathrm{c} 1,1,3) \& \operatorname{Mid}(\mathrm{c} 2,4,3) \& \operatorname{Mid}(\mathrm{c} 1,7,4)$

new2 $=\operatorname{Mid}(\mathrm{c} 2,1,3) \& \operatorname{Mid}(\mathrm{c} 1,4,3) \& \operatorname{Mid}(\mathrm{c} 2,7,4)$

'returning both as a concatenated string because dont know how to return 2 seperate vars... 'this string is split in half in the calling function to get 2 seperate strings

Crossover $=$ new $1 \&$ new 2

End Function

Function Mutate(c As String)

'at a random site, change a 0 to a 1 or a 1 to a 0

numBits $=$ Cells $(2,2)$. Value

random $=$ Rnd $*$ numBits

site $=$ Round (random, 0$)$

If site $=0$ Then

site $=1$

End If 
If Mid(c, site, 1) = "1" Then

Mutate $=\operatorname{Mid}(c, 1$, site -1$) \& 0 \& \operatorname{Mid}(c$, site $+1, \operatorname{Len}(c)-$ site $)$

Else

Mutate $=\operatorname{Mid}(c, 1$, site -1$) \& 1 \& \operatorname{Mid}(c$, site $+1, \operatorname{Len}(c)-$ site $)$

End If

\section{End Function}

Function isNotDuplicate(NewChild As String, rwIndex As Integer, colIndex As Integer, numChildren As Integer)

Dim ExistingChild As String

result $=$ True

For $\mathrm{i}=1$ To numChildren -1

ExistingChild $=$ Cells(rwIndex $+\mathrm{i}$, colIndex $)$. Value

If NewChild $=$ ExistingChild Then

result $=$ False

'duplicate test has failed, no point in continuing

'set $\mathrm{i}$ to the maximum to break the main loop and end the check

$\mathrm{i}=$ numChildren

End If

Next $i$

isNotDuplicate $=$ result

End Function 
; refined model of using gas kinetic numbers to simulate ion mobility and diffusion ; via stokes law with diffusion derived from kinetic jump statistics data ; This version supports multiple ion definitions as well as temperature, ; pressure, and bulk gas velocity fields (incorporated as array files)

SDS supports both electrostatic and magnetic fields

Electrostatic fields only:

Assuming test.pa0 is the electrostatic array then copy SDS.PRG

to a file called test.prg in the project directory

; Magnetic fields and electrostatic fields:

; copied

Assuming mag.pa0 is the magnetic array then SDS.PRG MUST be to a file called mag.prg in the project directory

; this version requires the existance of the following files:

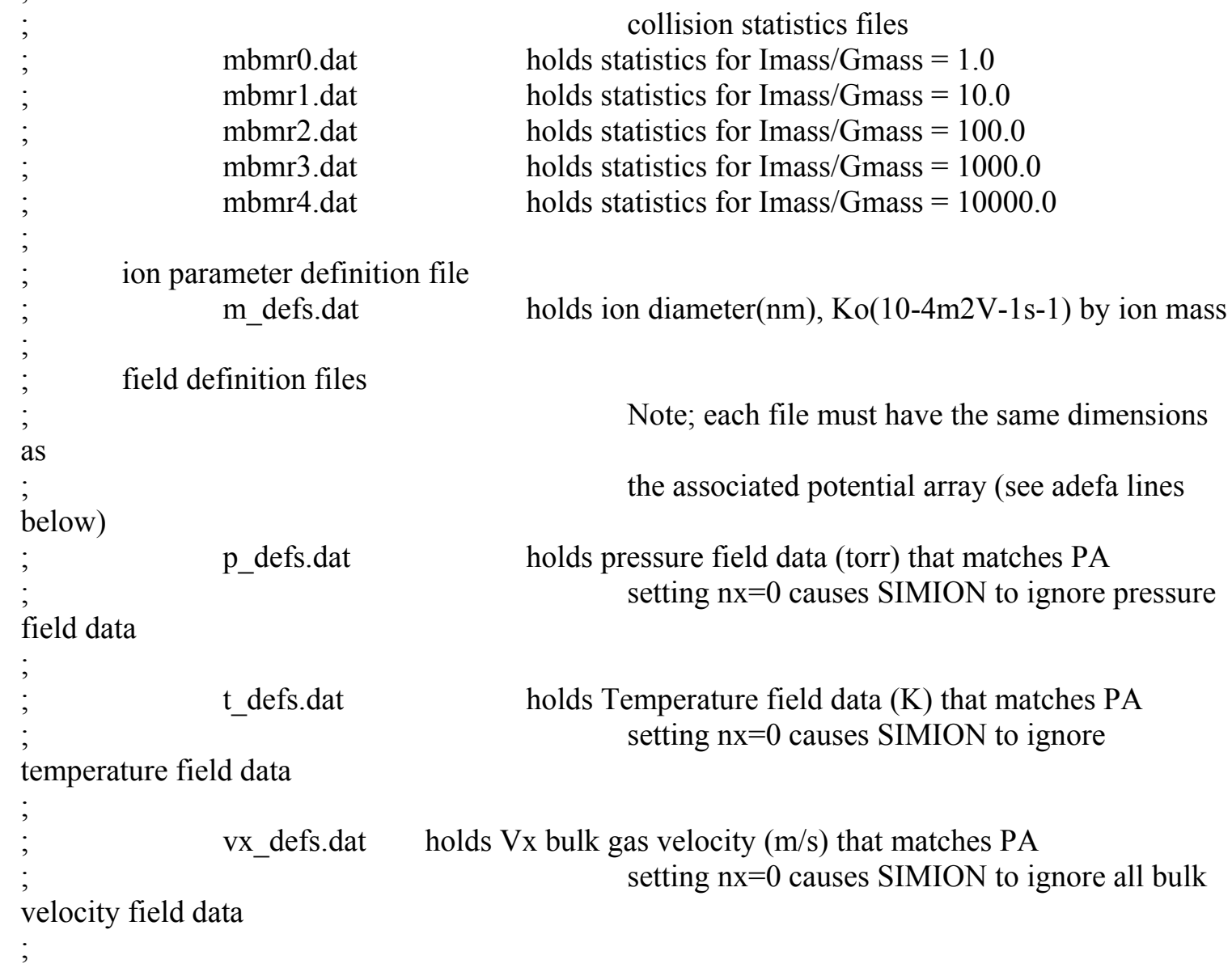

mbmr0.dat

mbmr1.dat

mbmr2.dat

mbmr3.dat

mbmr4.dat

ion parameter definition file m_defs.dat

holds ion diameter(nm), Ko(10-4m2V-1s-1) by ion mass

field definition files

Note; each file must have the same dimensions the associated potential array (see adefa lines holds pressure field data (torr) that matches PA setting $\mathrm{nx}=0$ causes SIMION to ignore pressure holds Temperature field data $(\mathrm{K})$ that matches PA setting $n x=0$ causes SIMION to ignore 


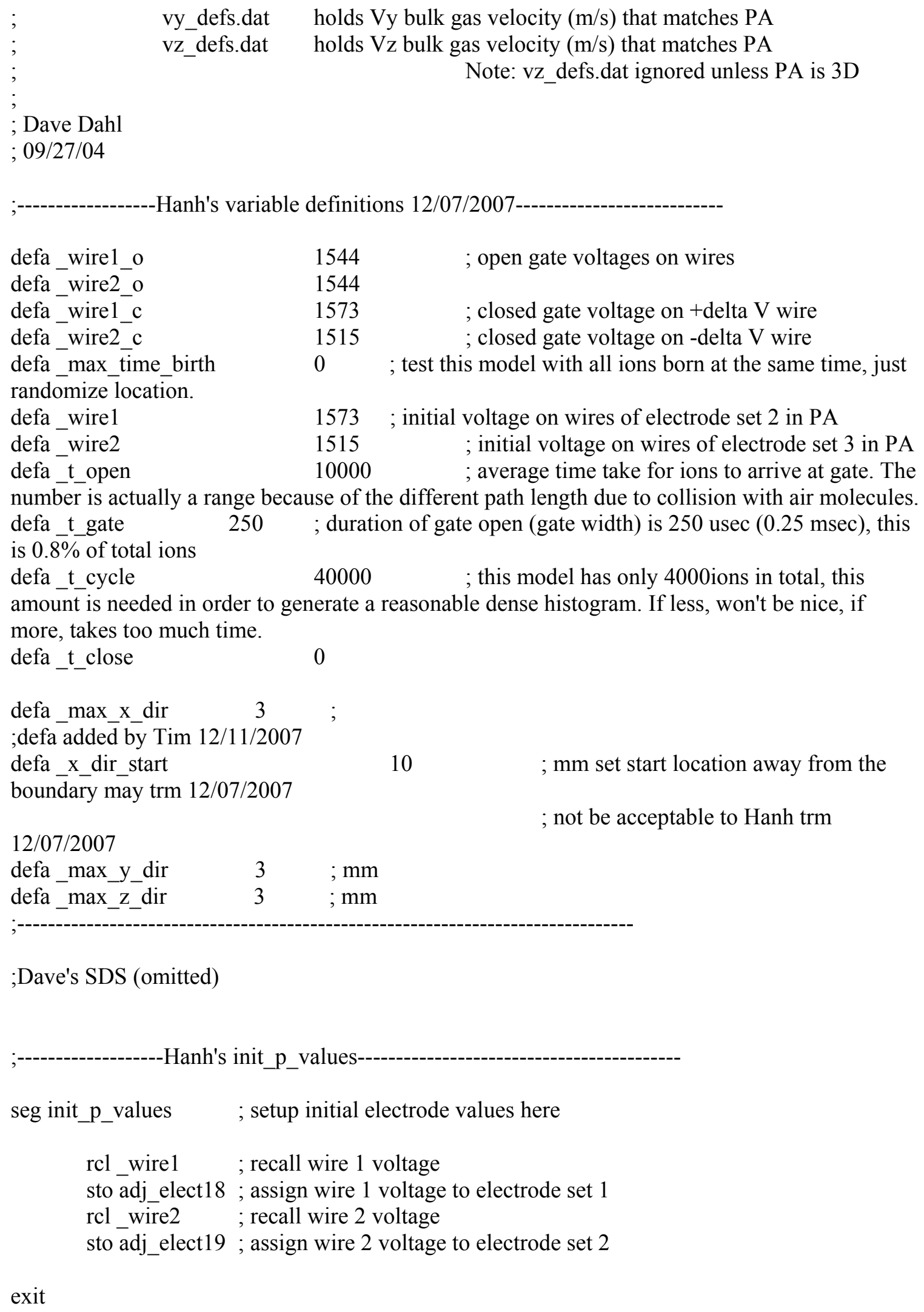




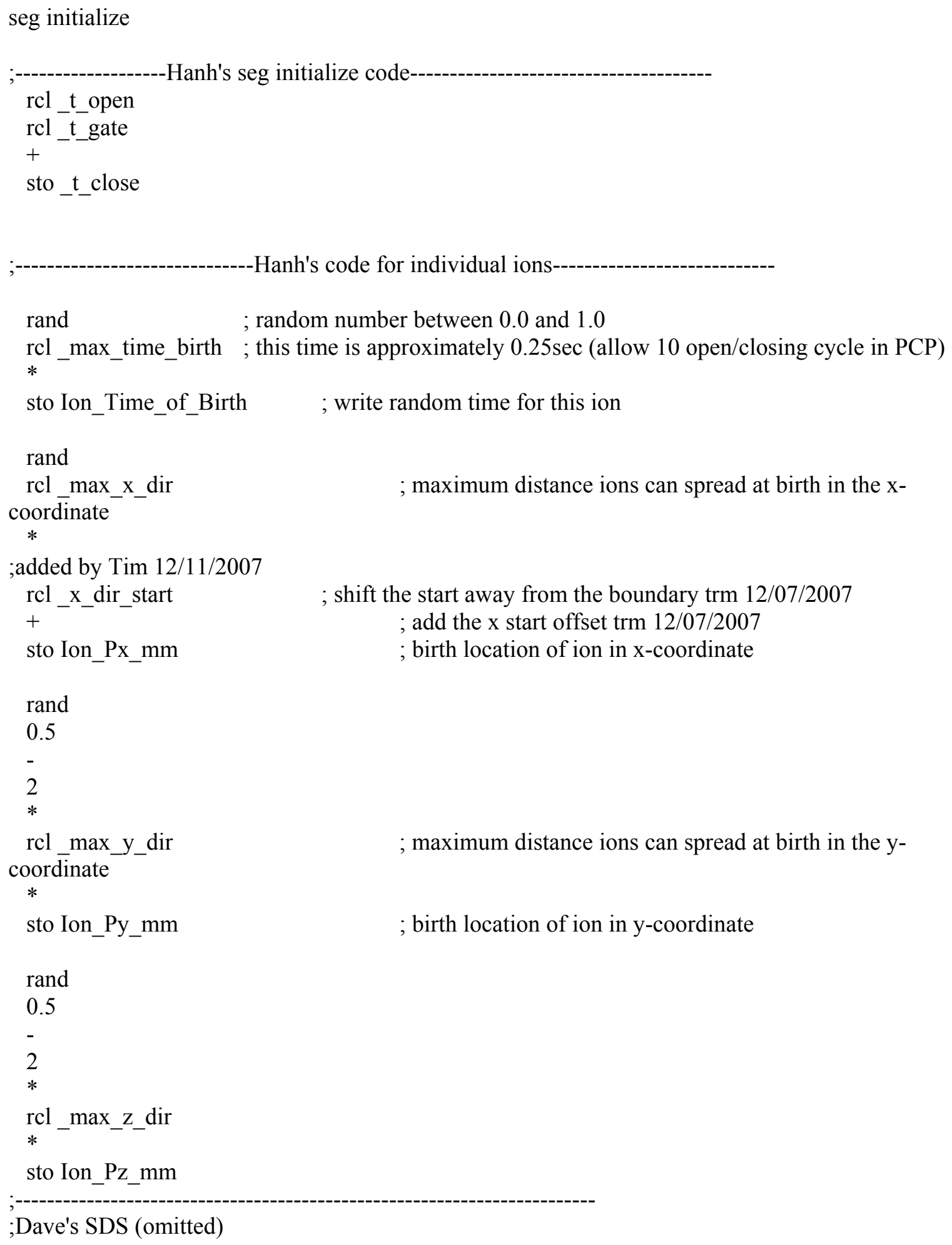




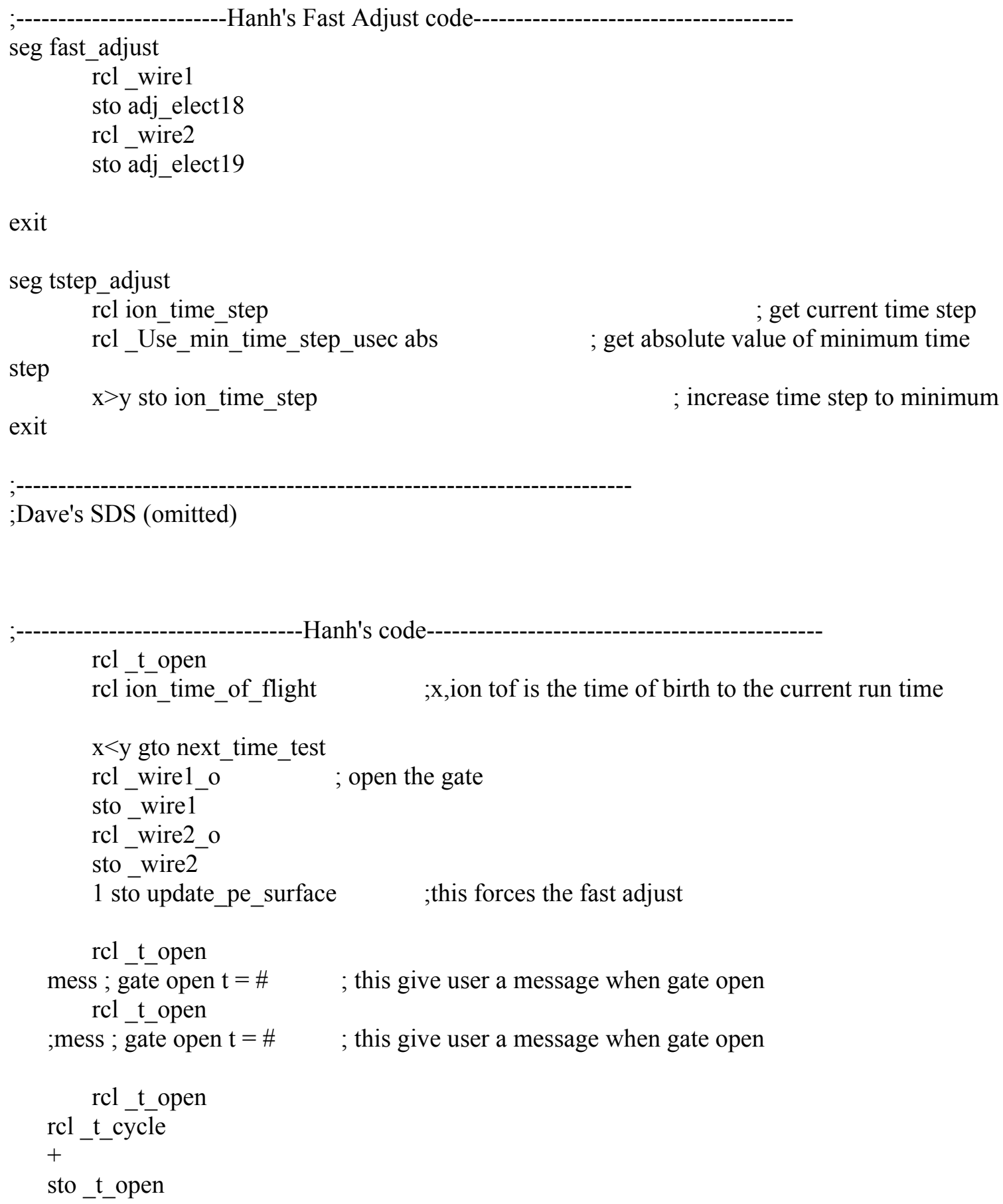




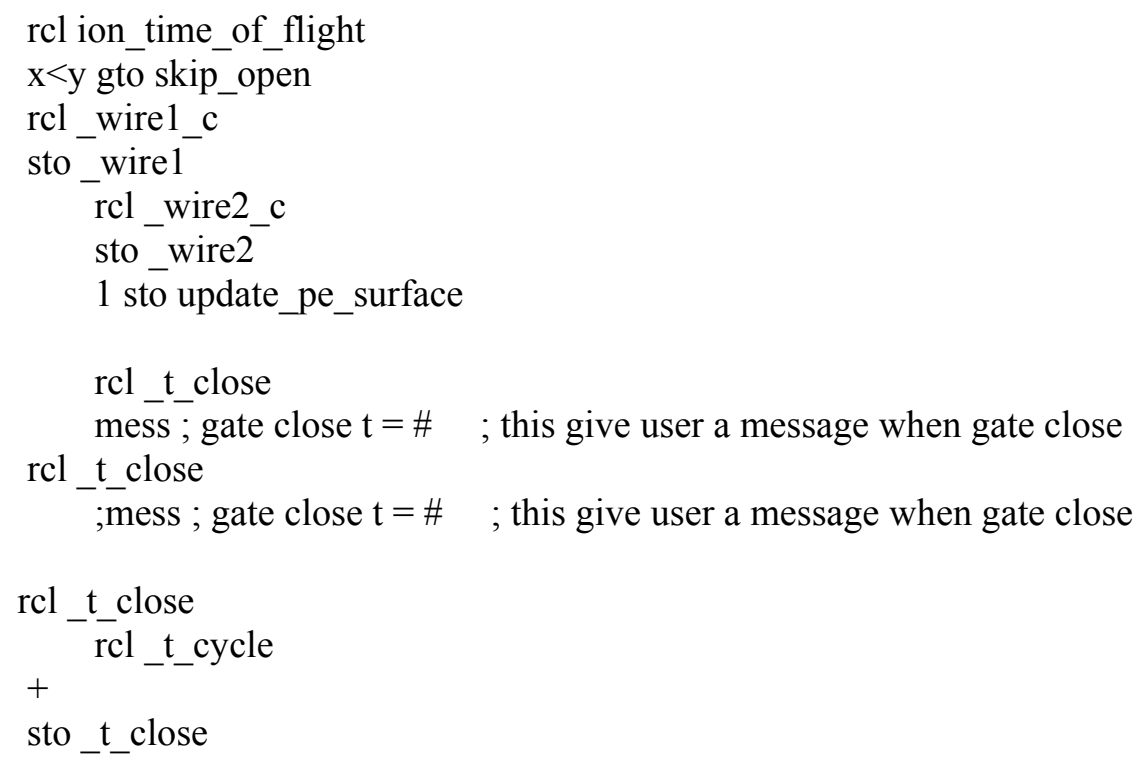

lbl skip_open

;Dave's SDS (omitted) 
Copyright clearance from Journal of Separation Sciences

Dear Customer

Thank you for your request.

We hereby grant permission for the requested use expected that due credit is given to the original source.

For material published before 2007 additionally: Please note that the author's permission is also required.

Please note that we only grant rights for a printed version, but not the rights for an electronic/ online/ web/ microfiche publication, but you are free to create a link to the article in question which is posted on our website (http://www3.interscience.wiley.com)

If material appears within our work with credit to another source, authorization from that source must be obtained.

$\Rightarrow$ You may use the version of the contribution as originally submitted for publication for an electronic presentation of the thesis. The contribution may not be updated or replaced with the published version. The version posted must contain a legend as follows: This is the pre-peer reviewed version of the following article: FULL CITE.

Credit must include the following components:

"Analysis of volatile components of drugs and explosives by solid phase microextractionion mobility spectrometry, Hanh Lai, Patricia Guerra, Monica Joshi, José R. Almirall, Journal of Separation Science 31(2): 402 - 412" Copyright Wiley-VCH Verlag GmbH \& Co. KGaA. Reproduced with permission.

With kind regards

Bettina Loycke

Bettina Loycke

Senior Rights Manager

Wiley-VCH Verlag GmbH \& Co. KGaA

Boschstr. 12

69469 Weinheim

Germany

Phone: +49 (0) 62 01- $606-280$

Fax: $\quad+49$ (0) $6201-606-332$

Email: rights@wiley-vch.de 
Copyright clearance from Journal of Analytical and Bioanalytical Chemistry

\section{SPRINGER LICENSE TERMS AND CONDITIONS}

Jan 14, 2010

This is a License Agreement between Hanh T Lai ("You") and Springer ("Springer") provided by Copyright Clearance Center ("CCC"). The license consists of your order details, the terms and conditions provided by Springer, and the payment terms and conditions.

All payments must be made in full to CCC. For payment instructions, please see information listed at the bottom of this form.

License Number

License date

Licensed content publisher

Licensed content publication

Licensed content title

Licensed content author

Licensed content date

Volume number

Issue number

Type of Use

Portion

Number of copies

Author of this Springer article

Order reference number

Title of your thesis / dissertation The construction and optimization of an ion mobility

Expected completion date

Estimated size(pages)

Total
2347651096232

Jan 14, 2010

Springer

Analytical and Bioanalytical Chemistry

Headspace sampling and detection of cocaine, MDMA, and marijuana via volatile markers in the presence of potential interferences by solid phase microextraction-ion mobility spectrometry (SPME-IMS)

Hanh Lai

Sep 1, 2008

392

1

Thesis/Dissertation

Full text

5

Yes and you are the sole author of the new work spectrometer for the analysis of explosives and drugs Feb 2010

200

0.00 USD

Terms and Conditions:

Introduction

The publisher for this copyrighted material is Springer Science + Business Media. By clicking "accept" in connection with completing this licensing transaction, you agree that the following terms and conditions apply to this transaction (along with the Billing and Payment terms and conditions established by Copyright Clearance Center, Inc. ("CCC"), at the time that you opened your Rightslink account and that are available at any time at http://myaccount.copyright.com).

\section{Limited License}

With reference to your request to reprint in your thesis material on which Springer Science and Business Media control the copyright, permission is granted, free of charge, for the use indicated in your enquiry. Licenses are for one-time use only with a maximum distribution equal to the number that you identified in the licensing process. This License includes use in an electronic 
form, provided it is password protected or on the university's intranet, destined to microfilming by UMI and University repository. For any other electronic use, please contact Springer at (permissions.dordrecht@springer.com or permissions.heidelberg@springer.com) The material can only be used for the purpose of defending your thesis, and with a maximum of 100 extra copies in paper. Although Springer holds copyright to the material and is entitled to negotiate on rights, this license is only valid, provided permission is also obtained from the (co) author (address is given with the article/chapter) and provided it concerns original material which does not carry references to other sources (if material in question appears with credit to another source, authorization from that source is required as well). Permission free of charge on this occasion does not prejudice any rights we might have to charge for reproduction of our copyrighted material in the future.

Altering/Modifying Material: Not Permitted However figures and illustrations may be altered minimally to serve your work. Any other abbreviations, additions, deletions and/or any other alterations shall be made only with prior written authorization of the author(s) and/or Springer Science + Business Media. (Please contact Springer at permissions.dordrecht@springer.com or permissions.heidelberg@springer.com)

Reservation of Rights Springer Science + Business Media reserves all rights not specifically granted in the combination of (i) the license details provided by you and accepted in the course of this licensing transaction, (ii) these terms and conditions and (iii) CCC's Billing and Payment terms and conditions.

Copyright Notice:

Please include the following copyright citation referencing the publication in which the material was originally published. Where wording is within brackets, please include verbatim. "With kind permission from Springer Science+Business Media: <book/journal title, chapter/article title, volume, year of publication, page, name(s) of author(s), figure number(s), and any original (first) copyright notice displayed with material $>$."

Warranties:

Springer Science + Business Media makes no representations or warranties with respect to the licensed material.

Indemnity You hereby indemnify and agree to hold harmless Springer Science + Business Media and CCC, and their respective officers, directors, employees and agents, from and against any and all claims arising out of your use of the licensed material other than as specifically authorized pursuant to this license.

No Transfer of License

This license is personal to you and may not be sublicensed, assigned, or transferred by you to any other person without Springer Science + Business Media's written permission.

No Amendment Except in Writing

This license may not be amended except in a writing signed by both parties (or, in the case of Springer Science + Business Media, by CCC on Springer Science + Business Media's behalf). 


\section{Objection to Contrary Terms}

Springer Science + Business Media hereby objects to any terms contained in any purchase order, acknowledgment, check endorsement or other writing prepared by you, which terms are inconsistent with these terms and conditions or CCC's Billing and Payment terms and conditions. These terms and conditions, together with CCC's Billing and Payment terms and conditions (which are incorporated herein), comprise the entire agreement between you and Springer Science + Business Media (and CCC) concerning this licensing transaction. In the event of any conflict between your obligations established by these terms and conditions and those established by CCC's Billing and Payment terms and conditions, these terms and conditions shall control.

\section{Jurisdiction}

All disputes that may arise in connection with this present License, or the breach thereof, shall be settled exclusively by the country's law in which the work was originally published. 


\section{Copyright clearance from International Journal of Mass Spectrometry}

This is a License Agreement between Hanh T Lai ("You") and Elsevier ("Elsevier") provided by Copyright Clearance Center ("CCC"). The license consists of your order details, the terms and conditions provided by Elsevier, and the payment terms and conditions.

All payments must be made in full to CCC. For payment instructions, please see information listed at the bottom of this form.

Supplier

Registered Company Number

Customer name

Customer address

License Number

License date

Licensed content publisher

Licensed content publication

Licensed content title

Licensed content author

Licensed content date

Volume number

Issue number

Pages

Type of Use

Portion

Format
Elsevier Limited

The Boulevard,Langford

Lane

Kidlington,Oxford,OX5

$1 \mathrm{~GB}, \mathrm{UK}$

1982084

Hanh T Lai

11200 SW 8th street

Miami, FL 33199

2362750217621

Feb 05, 2010

Elsevier

International Journal of Mass Spectrometry

The predictive power of SIMION/SDS simulation software for modeling ion mobility spectrometry instruments

Hanh Lai, Timothy R. McJunkin, Carla J. Miller, Jill R. Scott, José R. Almirall

15 September 2008

\section{0}

Thesis / Dissertation

Full article

Both print and electronic 
You are an author of the Elsevier article

Yes

Are you translating?

No

Order Reference Number

Expected publication date

Apr 2010

Elsevier VAT number

GB 494627212 


\section{HANH TUYET LAI}

December 28, 1981

June, 2005

\section{PUBLICATIONS}

1. H. Lai, P. Guerra, M. Joshi, J.R. Almirall*, Analysis of volatile components of drugs and explosives by using solid phase microextraction-ion mobility spectrometry, Journal of Separation Science (2008), 31: 402-412

2. H. Lai, T.R. McJunkin, C.L. Miller, J.R. Scott ${ }^{*}$, J.R. Almirall*, The Predictive Power of SIMION/SDS Simulation Software for Ion Mobility Spectrometer, International Journal of Mass Spectrometry (2008) 276:1-8

3. H. Lai, I. Corbin, J.R. Almirall*, Headspace Sampling and Detection of Cocaine, MDMA, and Marijuana via Volatile Markers in the Presence of Potential Interferences by SPME-IMS Journal of Analytical and Bioanalytical Chemistry (2008) 392:105-113

4. H. Lai, M. Magee, A. Leung, J.R. Almirall*, Identification of Volatile Chemical Signatures from Plastic Explosives by SPME-GC/MS and Detection by Ion Mobility Spectrometry, Journal of Analytical and Bioanalytical Chemistry (Submitted 2009)

5. P. Guerra, H. Lai, J.R. Almirall ${ }^{*}$, Analysis of the volatile chemical markers of explosives using novel solid phase microextraction coupled to ion mobility spectrometry, Journal of Separation Science(2008), 31: 2891-2898

6. M. Joshi, Y. Delgado, P. Guerra, H. Lai, J.R. Almirall*, Detection of odor signatures of smokeless powders using solid phase microextraction coupled to an ion mobility spectrometer, Forensic Sci. Int. (2009) 188: 112-118

7. S. Gura, P. Guerra-Diaz, H. Lai, J.R. Almirall*, Enhancement in sample collection for the detection of MDMA using a novel planar SPME (PSPME) device coupled to ion mobility spectrometry (IMS), Drug Test. Analysis (2009) 1:355-362

\section{SELECTED PRESENTATIONS}

Oral (presenting author):

1. American Academy of Forensic Science (International)

Systematic Optimization of Instrumental Conditions; IMS As a Model Device, Feb 2007, San Antonio, TX

2. Gordon Research Conference: Detection of Illicit Substances (International)

IMS; Successful Detection for Odor Signature Compounds of Illicit Drugs, Sept 2007, Big Sky, MN 
3. American Academy of Forensic Science (International)

Headspace Sampling \& Detection of Illicit Drugs by SPME-IMS, Feb 2008, Washington, DC

4. Scholarly Forum: Graduate Student Association (FIU)

The Evaluation of SIMION/SDS Simulation Software for IMS Instruments, April 2008, FIU

5. Florida Technology Innovation Showcase (Regional)

FIU Areas of Research and Pending Patents", Mar 2008, Orlando, FL

6. American Academy of Forensic Science (International)

Detection of Plastic Explosives by SPME-IMS via their volatile chemical markers, Feb 2009, Denver, CO

7. Scholarly Forum: Graduate Student Association (FIU)

Detection of Plastic Explosives by SPME-GC/MS and SPME-IMS, April 2009, FIU

8. Gordon Research Conference: Detection of Illicit Substances (International),

Detection of Plastic Explosives by SPME-IMS via their volatile chemical markers, June 2009, Les Diablerets, Switzerland

9. ICASS: Detection of Plastic Explosives by SPME-IMS via their volatile chemical markers, August 2009, Kingston, Canada

AWARDS

2009 Dissertation Year Fellowship, FIU Graduate School

$2009 \quad$ ICASS Conference Student Travel Award, CASS

$2008 \& 2009$ Scholarly Forum ( $3^{\text {rd }} \& 2^{\text {nd }}$ Prize winner), FIU Graduate Student Association

2008

2007

General Foundation Scholarship, FIU Graduate School

Best Poster Presentation Award, Gordon Research Conference

2006 \& 2007 Kauffman Entrepreneurial Doctoral Student Award, FIU Entrepr. Center

\section{PROFESSIONAL WORK EXPERIENCE}

2007 Summer Visiting scientist at Idaho National Laboratory, Idaho Falls, ID

2003-2004 Engineering Professional Experience Year at GE Power System, ON, Canada

\section{MEMBERSHIPS}

2007-2008 Graduate Student Association, Treasurer - FIU

- Member of a 7-student team. Practiced hands-on leadership skills serving 6000 graduate students of FIU and overseeing $\$ 145,000$ budget. Chaired 5 competitive workshops, and organized over 25 GSA annual events.

2007-2009 CAS Dean's Strategic Planning Committee, Graduate Reps. - FIU 